UCRL -53686

DE86 004184

URCL-53686

Distribution Category UC-34A

DISCLAIMER

This report was prepared as an account of work sponsored by an agency of the United States Government. Neither the United States Government nor any agency thereof, nor any of their employees, makes any warranty, express or implied, or assumes any legal liability or responsibility for the accuracy, completeness, or usefulness of any information, apparatus, product, or process disclosed, or represents that its use would not infringe privately owned rights. Reference herein to any specific commercial product, process, or service by trade name, trademark, manufacturer, or otherwise does net necessarily constitute or imply its endorsementh recommendation, or favoring by the United States Government or any agency thereof. The views and opinions of authors expressed herein do not necessarily state or reflect those of the United States Government or any agency thereof.

\title{
Pseudopotential Calculations and Photothermal Lensing Measurements of Two-Photon Absorption in Solids
}

\author{
Warren Travis White, III
}

(Ph.D. Thesis)

Manuscript date: November 4, 1985

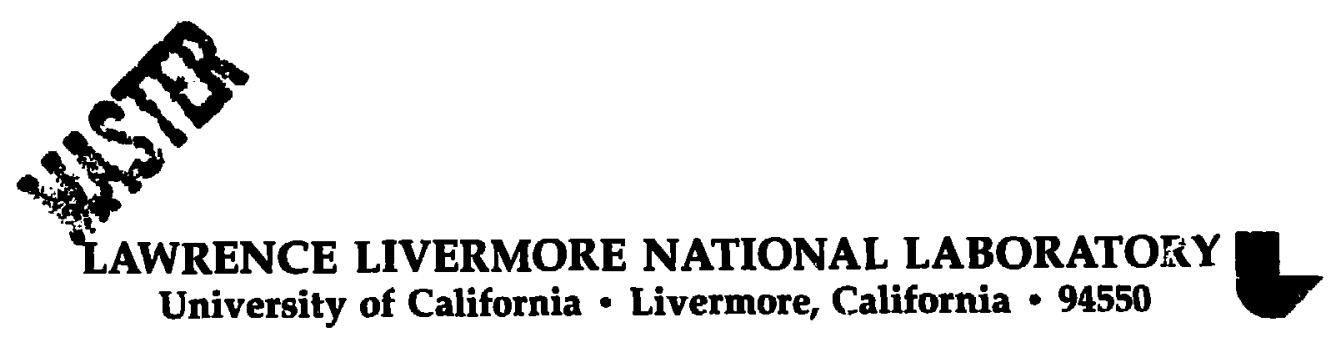

Available from: National Technical Information Service - U.S. Department of Commerce 5285 Port Royal Road - Springfield, VA 22161 - $\$ 20.50$ per copy • (Microfiche $\$ 4.50$ ) 
Pseudopotential Calculations and Fhotothermal Lensing Measurements of Two-Photon Absorption in Solids

By

WARREN TRAVIS WHITE, III

B.S. (United State Air Force Academy) 1971

M.S. (University of Rochester) 1972

\section{DISSERTATION}

Submitted in partial satisfaction of the requirements for the degree of

DOCTOR OF PHILOSOPHY

in

Engineering - Applied Science

in the

GRADUATE DIVISION

of the

UNIVERSITY OF CALIFORNIA

DAVIS

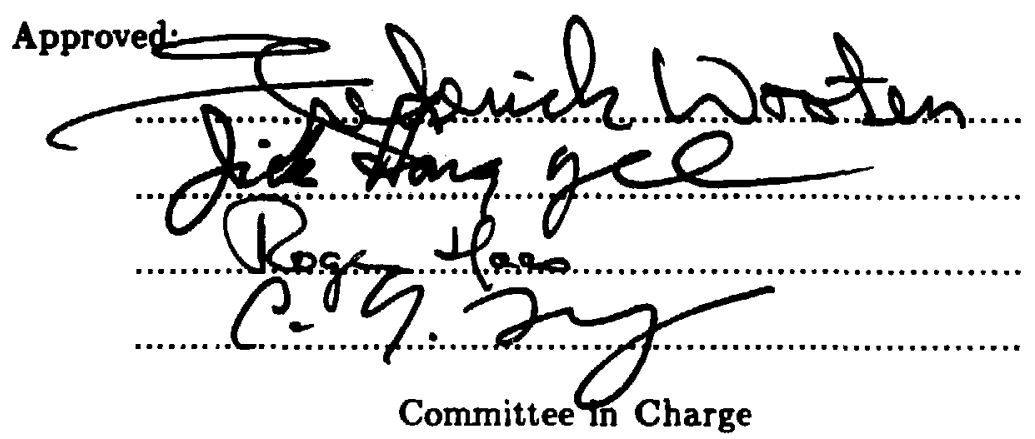

Deposited in the University Library 


\section{Abstract}

We have studied two-photon absorption in solids theoretically and experimentally. We have shown that it is possible to use accurate band structure techniques to compute two-photon absorption spectra within $15 \%$ of measured values in a wide band-gap material, $\mathrm{ZnS}$. The empirical pseudopotential technique that we used is significantly more accurate than previous models of two-photon absorption in zinc blende materials, including present tunneling theories (which are essentially parabolic-band results in disguise) and the nonparabolic-band formalism of Pidgeon et al. and Weiler. The agreement between our predictions and previous measurı ments allowed us to use $\mathrm{ZnS}$ as a reference material in order to validate a technique for measuring two-photon absorption that was previously untried in solids, pulsed dual-beam thermal lensing. With the validated technique, we examined nonlinear absorption in one other crystal (rutile) and in several glasses, including silicates, borosilicates, and one phosphate glass. Initially, we believed that the absorption edges of all the materials were comparable; however, subsequent evidence suggested that the effective band-gap energies of the glasses were above the energy of two photons in our measurement. Therefore, we attribute the nonlinear absorption that we observed in glasses to impurities or defects. The measured nonlinear absorption coefficients were of the order of a few $\mathrm{cm} / \mathrm{TW}$ in the glasses and of the order of 10 $\mathrm{cm} / \mathrm{GW}$ in the crystals, four orders of magnitude higher than in glasses. 


\section{Acknowledgments}

The author wishes to thank the Fannie and John Hertz Foundation, the Office of Basic Energy Sciences in the U. S. Department of Energy, and Lawrence Livermore National Laboratory for their generous support in this research. To Drs. John Emmett, John Holzrichter, and Howard Lowdermilk, special thanks for providing space and resources under the auspices of Y Program.

During the course of this project, the author was privileged to meet and work with many outstanding scientists and technicians. It is a pleasure to acknowledge these people for the help that they provided. To Serge Peluso, Tom Hindley, Jeanne Lynch, Mike Staggs, and Charmaine Vercimak, thanks for much skilled assistance and many good suggestions. Thanks, also, to those other individuals in Y Program who shared so freely their valuable time and experience, including Tony DeGroot, Geylord Larsen, Giles Peterson, Frank Rainer, Bill Warren and Drs. Mark Henesian and Stan Stokowski. To several individuals who provided special technical assistance, thanks: to Paul Hed, Dick Maney, and Eberhard Prochnow for polished optical samples; to Tom Hoheisel for thermal measurements; and to Charlie Farrell and Gordon Smith for X-ray crystallography.

It is with added pleasure that the author recognizes the contributions of these individuals, for their help was personal as well as professional: Bill Orvis, who introduced the author to practical energy band calculations; Dr. Doug Hall, who served as a sounding board for many ideas; Dr. Lee Smith, who introduced the author to two-photon absorptiori; and Prof. Alex Glass, who introduced the author to $n_{2}$. To Dr. David Milam, a master of practical sound thinking, thanks for much help and for Milam's Maxim ("the learning curve has only toes"). For sharing an incisive ability to reach a bottom line, the author gratefully recognizes $\mathrm{Dr}$. Marvin Weber. 
For typing Appendix F and portions of the manuscript in its early stages, thanks to Treva Mauch. For competently type setting the final draft, special thanks to Debbie Payne (and her family) and to Fran McFarland.

The author is especially indebted to his advisers, Drs. Charles G. Stevens and Jick $H$. Yee for the insight and guidance they provided, for their accessibility, and most of all for their frequent encouragement.

Finally, much gratitude and love to Sherry, my Proverbs 31 wife.

But thanks be to God, Who gives us the victory through our Lord Jesus Christ. 


\section{Contents}

Title Page $\ldots \ldots \ldots \ldots \ldots \ldots \ldots \ldots \ldots \ldots \ldots \ldots \ldots \ldots \ldots \ldots \ldots$

Abstract $\ldots \ldots \ldots \ldots \ldots \ldots \ldots \ldots \ldots \ldots \ldots \ldots \ldots \ldots$ ii

Acknowledgments $\ldots \ldots \ldots \ldots \ldots \ldots \ldots \ldots \ldots \ldots \ldots \ldots$ iii

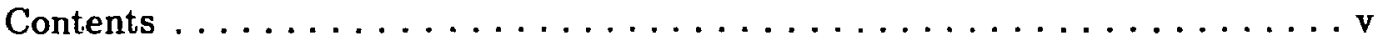

Chapter 1 - INTRODUCTION

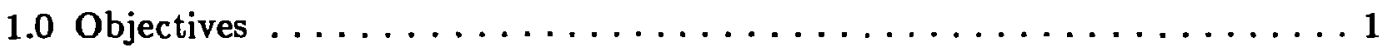

1.1 Background $\ldots \ldots \ldots \ldots \ldots \ldots \ldots \ldots \ldots \ldots \ldots \ldots \ldots \ldots \ldots \ldots \ldots \ldots \ldots \ldots$

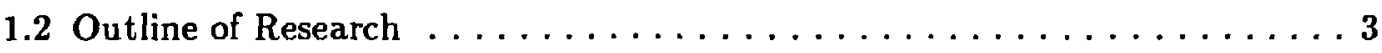

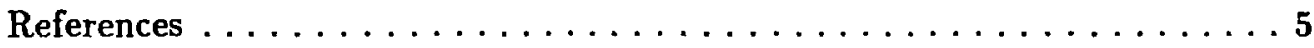

Chapter 2 - TWO-PHOTON ABSORPTION IN ZINC BLENDE

2.0 Introduction . . . . . . . . . . . . .

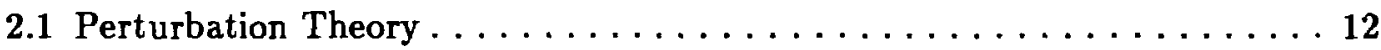

2.1.1 Intermediate States . . . . . . . . . . . . . . . . . 14

2.1.2 The Single-Intermediate-State Model . . . . . . . . . . . . 19

2.1.3 Self-Interaction Terms $\ldots \ldots \ldots \ldots \ldots \ldots \ldots \ldots \ldots \ldots \ldots 26$

2.2 Two-Photon Absorption in Zinc Blende Compounds . . . . . . . . 29

2.2.1 Nonparabolic Energy Bands $\ldots \ldots \ldots \ldots \ldots \ldots \ldots \ldots \ldots \ldots$

2.2.2 Intervalence- and Interconduction-Band Transitions $\ldots \ldots \ldots \ldots \ldots 40$

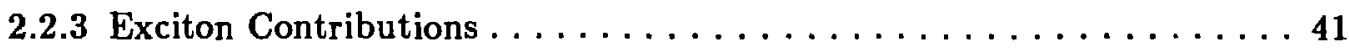

2.3 Tunneling Models of Two-Photon Absorption ............. 43

2.4 Nonlinear Optical Susceptibility Formalism $\ldots \ldots \ldots \ldots \ldots \ldots \ldots 44$

2.4.1 Polarization Dependence of $\beta \ldots \ldots \ldots \ldots \ldots \ldots \ldots \ldots \ldots \ldots \ldots \ldots \ldots \ldots \ldots$

2.4.2 Local-Field Corrections $\ldots \ldots \ldots \ldots \ldots \ldots \ldots \ldots \ldots \ldots \ldots \ldots$ 
2.5 Frequency Dependence of the Two-Photon Absorption Coefficient . . . . 54

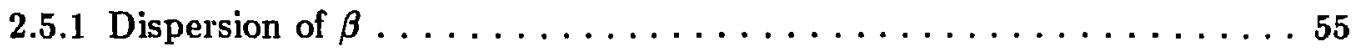

2.5.2 Relationship Between $\beta(\omega)$ and $\beta\left(\omega_{1}, \omega_{2}\right) \ldots \ldots \ldots \ldots 7$

2.6 Comparison Between Previous Theories and Experiments for $\mathrm{ZnS} \ldots \ldots 59$

2.7 Two-Photon Absorption in Optical Glasses $\ldots \ldots \ldots \ldots \ldots \ldots \ldots \ldots$

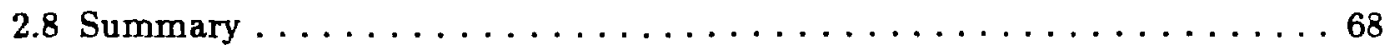

References ....................... 70

Chapter 3 - PSEUDOPOTENTIAL CALCULATION OF TWO-PFIOTON ABSORPTION IN $\mathrm{ZnS}$

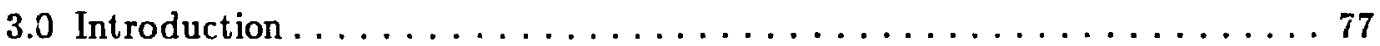

3.1 Determinantal Form of the One-Electron Wave Equation $\ldots \ldots \ldots \ldots 78$

3.2 Local Pseudopotential Approximation with Spherical Form Factors . . . . 80

3.3 Pseudopoiential Calculations of Energy Bands in $\operatorname{ZnS} \ldots \ldots \ldots \ldots . .83$

3.4 Calculation of Two-Photon Absorption in $\mathrm{ZnS} \ldots \ldots \ldots \ldots \ldots 2$

3.4.1 Mechanics of Calculating $\beta(\omega) \ldots \ldots \ldots \ldots \ldots \ldots$

3.4.2 Comparison of the Pseudopotential to Other Theoretical Methods . . . 99

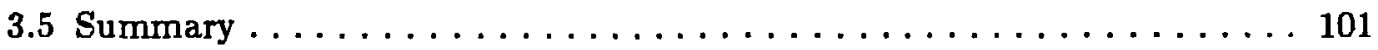

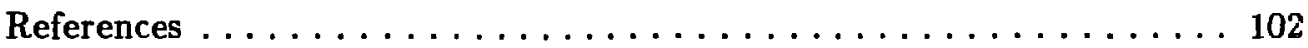

Chapter 4 - DUAL-BEAM PULSED THERMAL LENSING

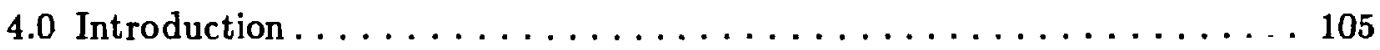

4.1 Geometrical Optics Model of Thermal Lensing . . . . . . . . . . 108

4.2 Thermal Lensing of Two Gaussian Beams . . . . . . . . . . . . . 112

4.3 Spherical Aberration in a Pulsed Thermal Lens . . . . . . . . . . 121

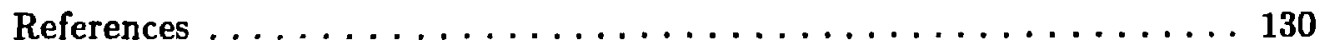


Chapter 5 - EXPERIMENTAL RESULTS

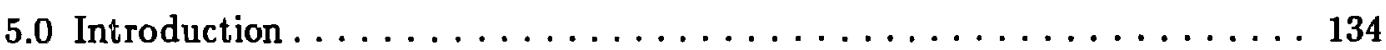

5.1 Apparatus and Procedures $\ldots \ldots \ldots \ldots \ldots \ldots \ldots \ldots \ldots \ldots \ldots$

5.1.1 The Pump Beam $\ldots \ldots \ldots \ldots \ldots \ldots \ldots \ldots \ldots \ldots \ldots \ldots$

5.1.2 The Probe Beam $\ldots \ldots \ldots \ldots \ldots \ldots \ldots \ldots \ldots \ldots \ldots$

5.1.3 Photodetectors and Signal Averagers . . . . . . . . . . . 142

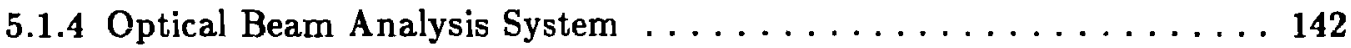

5.1.5 Transient Digitizer $\ldots \ldots \ldots \ldots \ldots \ldots \ldots \ldots \ldots \ldots \ldots \ldots \ldots$

5.1 .6 Electronic Timing $\ldots \ldots \ldots \ldots \ldots \ldots \ldots \ldots \ldots$

5.1.7 Optical Energy Calibration $\ldots \ldots \ldots \ldots \ldots \ldots \ldots \ldots \ldots \ldots$

5.1 .8 Optical Alignment $\ldots \ldots \ldots \ldots \ldots \ldots \ldots \ldots \ldots \ldots \ldots \ldots$

5.1.9 Photothermal Linearity . . . . . . . . . . . . . . 147

5.1 .10 Data Reduction $\ldots \ldots \ldots \ldots \ldots \ldots \ldots \ldots \ldots \ldots \ldots$

5.2 Results for $\mathrm{ZnS} \ldots \ldots \ldots \ldots \ldots \ldots \ldots \ldots \ldots \ldots \ldots \ldots \ldots$

5.3 Results for $\mathrm{TiO}_{2}$ (Rutile) $\ldots \ldots \ldots \ldots \ldots \ldots \ldots \ldots \ldots \ldots \ldots \ldots \ldots \ldots \ldots$

5.4 Results in Jxide Glasses . . . . . . . . . . . . . . . 161

5.4.1 Glass Compositions . . . . . . . . . . . . . . . . 169

5.4.2 Measurement Technique in Glasses $\ldots \ldots \ldots \ldots \ldots \ldots \ldots \ldots$

5.5 Calibration of Thermal Lensing Measurements with Doped Samples . . . 171

5.5.1 Effective Thermo-optic Coefficients .............. 171

5.5.2 Determination of Effective Thermal Conversion Efficiency $(1-\eta) \ldots \ldots 175$

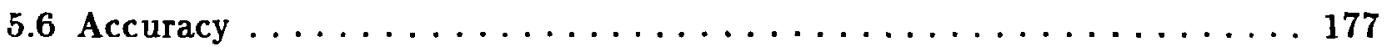

5.7 Summary $\ldots \ldots \ldots \ldots \ldots \ldots \ldots \ldots \ldots \ldots \ldots \ldots \ldots \ldots$

References ........................ 182 
Chapter 6 - CONCLUSIONS AND SUMMARY

6.0 Conclusions and Summary $\ldots \ldots \ldots \ldots \ldots \ldots \ldots \ldots \ldots \ldots \ldots$

References .......................... 188

Appendix A - Bloch's Determinantal Form of Schrödinger's

Equation in Crystals . . . . . . . . . . . . . . . . . . . 189

Appendix B -Reciprocal Lattice Vectors of a Face-Centered Cubic Lattice . 193

Appendix C -First Brillouin Zone of the FCC Lattice $\ldots \ldots \ldots \ldots \ldots$

Appendix D -Indirect Two-Photon Absorption in $\mathrm{ZnS} \ldots \ldots \ldots \ldots$

Appendix E -Boundary Conditions on the Thermal Lens . . . . . . . . 208

Appendix $\mathrm{F}$-Thermal Lensing Bibliography $\ldots \ldots \ldots \ldots \ldots$

List of Figures

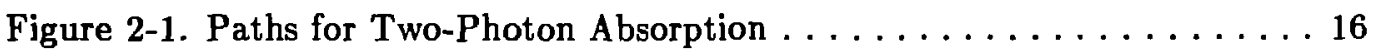

Figure 2-2. Equivalence of $(A)$ the Distinguishable- and $(B)$ the

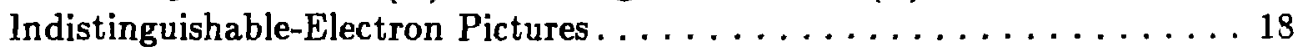

Figure 2-3. Energy Levels in Braunstein's SIS Model . . . . . . . . . . . . 22

Figure 2-4. Band Structure in Basov's Model . . . . . . . . . . . 27

Figure 2-5. Two-Photon Transitions in Yee's Model . . . . . . . . . . . . 31

Figure 2-6. Measured Two-Photon Absorption Spectra in ZnS . . . . . . 61

Figure 2-7. Comparison of Theoretical and Measuren Two-Photon

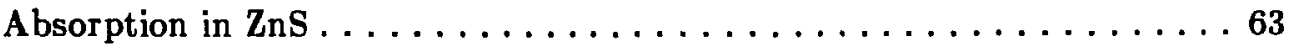

Figure 3-1. Pseudopotential Form Factors for Zinc and Sulfur . . . . . . 85

Figure 3-2. Energy Bands Along [100] in Cubic ZnS . . . . . . . . . 88

Figure 3-3. Imaginary Part of the Linear Dielectric Constant . . . . . . 90

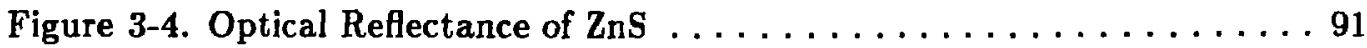

Figure 3-5. Pseudopotential Calculation of $\beta \ldots \ldots \ldots \ldots . \ldots \ldots$

Figure 3-6. Comparison of Experiment and Pseudopotential Values of $\beta$. . . . 94

Figure 3-7. Comparison of Other Theories to the Pseudopotential Results . . 100

Figure 4-1. Dual-Beam Thermal Lensing . . . . . . . . . . . . . 106

Figure 4-2. Key Parameters of the Probe Beam . . . . . . . . . . . . 111

Figure 4-3. Effect of Sample Position upon Thermal Lensing Signal . . . . . 113

Figure 4-4. Lens with a Gaussian-Shaped Profile . . . . . . . . . . 122 


\section{Chapter 1}

\section{INTRODUCTION}

\subsection{Objectives.}

The objectives of this research are (1) to investigate the applicability of pseudopotential techniques to calculating two-photon absorption (2PA) in a wide band-gap solid, (2) to validate a new experimental technique for measuring two-photon absorption in solids, photothermal lensing (PTL), and (3) to use PTL to measure nonlinear absorption in some laser glasses that have not previously been tested.

\subsection{Background.}

Nonlinear optical absoiption in solids has been studied extensively because of both its basic scientific interest and its engineering importance. Two-photon transitions hav: different selection rules and mix polarizations differently than onephoton transitions. In a sense, two-photon spectroscopy complements one-photon spectroscopy as a basic probe of matter. In materials which exhilit no one-photor absorption, optical absorption can still occur by two-photon processes. Consequently, nonlinear absorption is a major practical limitation in the design of optics for high-intensity laser systems, which of necessity use highly transparent materials. This pertains not only to large pulsed systems like fusion lasers, ${ }^{1}$ but it also pertains to very small-bore systems such as fiber optic transmission lines. ${ }^{2}$

Although much already is known about two-photon absorption in solids, much remains to be learned. In a given material, reported values of two-photon absorption coefficients often vary by orders of magnitude from one measurement to another. ${ }^{3}$ Theories likewise vary among themselves. ${ }^{4}$ Even when theories agree reasonably well among themselves, ${ }^{5}$ they may be unable to distinguish between valid and invalid experimental results. This has been the case for large band-gap zinc blende 
materials like $\mathrm{ZnS}$, for instance. Thus a need still exists for definitively modeling and measuring two-photon absorption coefficients - even in materials such as zinc blende compounds, which have been widely studied.

Further, entire classes of materials, namely amorphous insulators and semiconductors, remain largely uninvestigated. ${ }^{6}$ In particular, only a handful of highly transparent optical glasses have been tested for two-photon absorption. ${ }^{6,7}$ This is somewhat surprising, considering the ubiquity of glass components in high-intensity laser systems. It is even more surprising when one realizes that, for those glasses which have been tested, the intrinsic two-photon absorption coefficients tend to be extremely small. Previously measured glasses have, at a given wavelength, coeffcients which are typically two orders of magnitude smaller than the same coefficients of $c_{j}$ stals of purportedly comparable energy band gaps. ${ }^{1}$

Measurements of 2PA in highly transparent solids require sensitive techniques, because two-photon absorption is a weak effect - particularly in glasses. The weakness of the effect cannot be compensated by arbitrarily increasing either the optical intensity or the sample length, because laser-induced damage and self-focusing limit the allowable intensity in a sample.

One measurement technique with sensitivity adequate to measure 2PA in highly transparent solids appears to be photothermal lensing (PTL). ${ }^{7}$ This technique will be described later. For now, the two most important aspects of photothermal lensing are, first, it is sensitive to weak optical absorption and, second, it is a novel way to quantify two-photon absorption in solids. (Recent measurements by us are the only reported atternpts to use PTL to measure two-photon absorption in solids. ${ }^{7}$ )

Part of validating the use of PTL to measure two-photon absorption in an arbitrary solid is to measure two-photon absorption in a solid that is well understood. In this study, a well understood solid not only denotes a solid in which two-photon 
absorption coefficients have been measured by other mears; it also denotes a solid whose two-photon absorption can be modeled accurately. Because of the variation in measured values of two-photon absorption coefficients for a given solid, theoretical predictions mus' be used where possible to check the validity of measurements. However, detailed, reliable models often do not exist, due to approximations in modeling electronic states of solids. It is therefore interesting to determine if current predictions of two-photon absorption in solids can be improved by using a more accurate model of electronic states. Specifically, can empirical pseudopotential methods, which have been spectacularly successful in modeling the linear optical properties of many crystals, successfully model nonlinear absorption as well? Work by Vaidyanathan et al. ${ }^{8}$ suggests that this might ke the case. However, those workers have reported only calculations at a discrete point, which is insufficient to show the ovsrall goodness of pseudopotential calcularions of two-photon absorption.

\subsection{Outline of Research.}

The body of this dissertation has five more chapters and several app ${ }^{\wedge}$ dices.

Chapter 2 treats the theory of two-photon absorption in solids, particularly zinc blende materials. The prevailing models of two-photon absorption are critically reviewed and compared to existing measurements. In addition, two-photon absorption in optical glass is discussed, without regard to detailed electronic structure.

Chapter 3 discusses the empirical pseudopotential method. A local potential with spherical atomic form factors is used to estimate the single-electron wave functions in $\mathrm{ZnS}$. The chapter shows that the empirical oseudopotential method can accurately predict two-photon absorption coefficients in $\mathrm{ZnS}$.

Chapter 4 discusses the theory of dual-beam photothermal lensing, drawing largely from Twarowski and Kliger's theory. ${ }^{9}$ The essential result of that chapter is a simple, revised model that, for the first time, properly accounts for the finite 
widths of the two beams.

Chapter 5 discusses our thermal lensing measurement of two-photon absorption in solids. The main result of the chapter is to show that thermal lensing is indeed a viable technique for measuring two-photon absorption in solids - even in highly transparent glasses in which the coefficients of nonlinear absorption are on the order of $1 \mathrm{~cm} / \mathrm{TW}$. Results from two crystals $\left(\mathrm{ZnS}\right.$ and $\left.\mathrm{TiO}_{2}\right)$ and several glasses are presented.

The final chapter summarizes our results.

The appendices contain various derivations and a bibliography on the rapidly expanding field of thermal lensing. 


\section{$\underline{\text { References }}$}

1. W. L. Smith, "Two-photon absorption and solarization in UV glasses," Laser Program Annual Report, UCRL-50021-81, E. V. George, scientific editor, 7.2024 (University of California, Lawrence Livermore National Laboratory Aug. 1982); ibid., UCRL-50021-82, C. D. Hendricks, scientific editor, 7.34-38 (Aug. 1983).

2. R. H. Stolen and C. Lin, "Two-photon and two-step absorption in glass optical waveguide, in Optical Properties of Highly Transparent Solids, S. S. Mitra and B. Bendow, editors, 307-15 (Plenum Press, New York 1975).

3. J. H. Bechtel and W. L. Smith, "Two-photon absorption in semiconductors with picosecond laser pulses," Phys. Rev, B 13(8), 3515-22 (15 Apr 1976).

4. A. Vaidyanathan, T. Walker, A. H. Guenther, S. S. Mitra, and L. M. Narducci, "Two-photon absorption in several direct-gap crystals," Phys. Rev. B 21(2), 743-48 (15 Jan. 1980).

5. A. Vaidyanathan, A. H. Guenther, and S. S. Mitra, "Two-photon absorption in direct-gap crystals - an addendum," Phys. Rev. B 22(12), 6480-83 (15 Dec. 1983).

6. W. L. Smith, "Two-photon absorption in condensed media," to be published in CRC Handbook of Laser Science and Technology, Volume III, M. J. Weber, editor (CRC Press, 1985).

7. W. T. White, III, M. A. Henesian, and M. J. Weber, "Photothermal lensing measurements of two-photon absorption and two-photon-induced color centers in borosilicate glasses at $532 \mathrm{~nm},{ }^{,}$to be published in J. Opt. Soc. Am. B2(9), (Sept. 1985).

8. A. Vaidyanathan, A. H. Guenther, and S. S. Mitra, "Band structure calculations of the two-photon absorption coefficients of GaAs, InP, CdTe, and ZnSe," Phys. 
Rev. B 24(4), 2259-62 (15 Aug. 1981).

9. A. J. Twarowski and D. Kliger, "Multiphoton absorption spectra using thermal blooming," Chem. Phys. 20, 253-64 (North Holland Publishing Co., Amsterdam, 1977). 
Chapter 2

\section{TWO-PHOTON ABSORPTION IN ZINC BLENDE}

\subsection{Introduction.}

This chapter reviews the theory of two-photon absorption and compares some existing measurements and theories. The chapter is organized in eight more sections. Section 2.1 describes time-dependent perturbation theory. Section 2.2 applies the theory to zinc blende materials. Within the framework of Kane's four-band model of the electronic states, new theoretical results are presented for those zinc blende materials that have weak spin-orbit splitting of the valence bands. Section 2.3 discusses the tunneling theory of two-photon absorption. Section 2.4 discusses the nonlinear-susceptibility formalism, including local-field considerations and polarization effects. Section 2.5 discusses the frequency-dependence of two-photon absorption. Section 2.6 applies the theories of the preceeding sections to zinc blende. Section 2.7 discusses two-photon absorption in optical glass, and Section 2.8 summarizes the chapter.

Two-photon absorption is a process in which the simultaneous absorption of two photons excites a material system from one stationary state to another. It was first predicted by Göppert-Mayer ${ }^{1}$ in 1931, but it was not observed until 1949, when Hughes and Grabner ${ }^{2}$ performed radio-frequency measurements on molecular beams of RbF. Two-photon absorption in solids was first observed in 1961 after the development of the laser, when Kaiser and Garret ${ }^{3}$ reported on two-photon-induced fluorescence of $\mathrm{Eu}^{2+}$ impurities in $\mathrm{CaF}_{2}$. Since 1961, two-photon absorption has been studied for a variety of reasons: as a unique optical probe of the symmetry of matter, ${ }^{4}$ as a fundamental limitation to the transparency of solids, ${ }^{5}$ as a mechanism for pumping semiconductor lasers, ${ }^{6}$ and as a probe of the x-ray region. ${ }^{7}$ Several 
reviews survey the research on two-photon absorption. ${ }^{8-12}$ Of special interest is Smith's recent Libliography of experimental data. ${ }^{13}$

Broadly speaking, only two classes of two-photon absorption exist, direct and indirect. During direct two-photon absorption, one photon excites a material system to an intermediate state, and then before the system can relax, a second photon excites the system to a final state. When the intermediate state is a well-defined stationary state of the unperturbed material system (a "real state"), the transition is called a two-step absorption. When the intermediate state is an admixture of states (a "virtual state"), the transition is called simply a two-photon absorption. During two-photon absorption, high photon fluxes are required to ensure a reasonable chance of exciting the material while it is in the intermediate state.

Indirect two-photon absorption is a frequency-conversion process. Two electromagnetic waves first mix nonlinearly in matter. Then the resulting sum- or difference-frequency wave is absorbed by the matter. An example is second-harmonic generation followed by one-photon absorption of the second harmonic wave. By symmetry, indirect two-photon absorption can occur only in acentric materials.

"Indirect two-photon absorption" should not be confused with "two-photon absorption in indirect-gap sclids." In the latter process, the word indirect implies that optical transitions are phonon-assisted. (Such transitions require either the absorption or the emission of a vibrational quantum in order to conserve both energy and momentum.) In the former process, indirect refers only to the fact that optical frequency conversion must occur before photons can be absorbed. The fact that indirect two-photon absorption occurs in a material says nothing about whether or not the material has an indirect energy gap. Appendix D discusses indirect two-photon absorption further, showing that it is unimportant in this work by comparison to direct two-photon absorption. 
Two-photon absorption is in some sense no different than one-photon absorption or any other type of optical absorption. In all cases, a temporary, external optical field mixes the natural quantum states of a material system. When the field stops aciing upon the material, then the material system must end up in one of its natural, stationary states. By conservation of energy, any increase in the energy of the material must derive from the optical field. Loosely, if the energy difference between the final and the initial states of the matter equals the energy of one photon, then the absorption is called a one-photon absorption. If it equals the energy of two photons, then the absorption is called two-photon absorption. If it equals the energy of $n$ photons, then the absorption is called $n$-photon absorption.

This chapter reviews the major formalisms that are used to describe two-photon absorption in solids, namely the time-dependent perturbation approach, electron turneling theory, ${ }^{14}$ and the nonlinear-susceptibility formalism. ${ }^{15,16}$ The first two descriptions are generally approached as microscopic models and the last one as a macroscopic model. The independent, one-electron approximation is used throughout this work.

For concreteness, the theory will be applied to a specific class of solids, the zinc blende materials. These important materials have been widely studied, and so it is possible to estimate the precise value of their two-photon absorption coefficients. Of particular interest is zinc sulfide. It has a wide band-gap energy and is more transparent at visible wavelengths than most zinc blende materials. It therefore is optically more like the transparent materials used in high-power optics than are its sister compounds. Since intrinsic two-photon absorption in insulators (particulary amorphous insulators) is not well understood, it is hoped that a careful examination of $\mathrm{ZnS}$ will provide some insight into the nonlinear optical properties of highly transparent media, including glasses. 
Another reason to scrutinize $\mathrm{ZnS}$ is o resolve a continuing dispute in the literature about how best to model two-photon absorption in zinc blende materials. Does a tunneling model predict the most accurate (i.e., experimentally observed) values, or is a perturbation theory better? Is the parabolic-band approximation adequate, or should more realistic band structures be used? One group of workers rlaims that tunneling models give comparable results to perturbation theory models. ${ }^{12}$ Another worker claims that perturbation theory predictions are much lower than those of the tunneling model. ${ }^{17}$ Some groups have claimed that the results of parabolic-band calculations are grossly different than the results of slightly non-parabolic bands. ${ }^{12,18}$ Careful examination of previous work shows that the apparent differences among models are almost always the result of modeling errors - either subtle mathematical errors or misapplications of physical principles. In addition, when validating their theories, previous workers often have used a single measured value at a discrete frequency. Since the coefficient of two-photon absorption rises steeply near the absorption edge, slight errors in the models can appear to yield significant discrepancies between experiment and theory. For a more thorough evaluation, one should compare the theories to spectra of two-photon absorption, not to isolated points. Two-photon absorption has been measured over a wider spectral rarige in $\mathrm{ZnS}$ than in any other zinc blende compound. Therefore, $\mathrm{ZnS}$ is especially useful as a touchstone for validating theories of nonlinear absorption.

Finally, it is necessary to understand two-photon absorption in $\mathrm{ZnS}$, because this material will be used to help validate the themal lensing technique of Chapters 4 and 5 . In particular polycrystalline, chemically vapor depositied $\mathrm{ZnS}$, which is obtainable in high purity and large sizes, will be tested.

Two caveats should be mentioned now at the outset of this work. First, the 
best available nonlinear absorption spectra for $\mathrm{ZnS}$ are for the hexagonal wurtzite phase, not for the cubic zinc blende phase. Second, the broadest range of data for wurtzite, Bae, Kim and Song's measurements, ${ }^{19}$ are only relative measurements. Both of these caveats will be resolved in the next chapter, where the results of detailed calculations of two-photon absorption are given for $\mathrm{ZnS}$. For the moment, we only suggest why these issues are not obstacles.

Cohen has argued that the similarity between the linear optical spectra of wurtzite and zinc blende suggests a fundamental similarity between their electronic structures. ${ }^{20}$ In a way, both are the same, since the fundamental structural unit in each is a tetrahedron of $\mathrm{S}$ ions surrounding a $\mathrm{Zn}$ ion. The teirahedra are arrayed only slightly differently in wurtzite than in zinc blende. From a tight-binding view, if the polarizabilities of the tetrahedra dominate the optical response of the crysials, then one might argue that gross spectral features should be similar between the two phases of ZnS, particularly for light polarized along the cubic (111) axis of zinc blende and along the hexagonal (001) axis (c-axis) of wurtzite. Galanin and Chizhikova have extended this argument to apply to two-photon absorption. ${ }^{21}$ That is, they posit that wurtzite and zinc blende should have similar coefficients of two-photon absorption. This conjecture will be validated by subsequent chapters in this work.

With respect to the second caveat, that of calibrating the relative two-photon absorption measurements of Bae et al., two comments are in order. First, these uncalibrated data can be referenced to independent measurements by other workers, although the latter are of limited spectral extent. Second, the calibration can be validated against the calculations to appear in Chapter 3. It will be seen that the agreement between the theory and the previously uncalibrated data is excellent. For now we shall assume that the data are well calibrated. 


\subsection{Perturbation Theory.}

Second-order, time-dependent perturbation theory gives the following expression for the total rate of two-photon transitions of a quantum mechanical system illuminated by monochromatic radiation of frequency $\omega$ :

$$
\begin{gathered}
W^{(2)}=\frac{2 \pi}{\hbar} \sum_{f} \sum_{i}\left|\sum_{n} \frac{H_{f n}^{\prime} H_{n i}^{\prime}}{E_{n}-E_{i}-\hbar \omega}\right|^{2} \\
\times \delta\left(E_{f}-E_{i}-2 \hbar \omega\right) .
\end{gathered}
$$

The subscripte $i, f$, and $n$ denote the initial, final, and intermediate states of the system, respectively. The terms $H_{i j}^{\prime}$ are transition matrix elements between quantum states $\mathrm{i}$ and j. $E_{i}, E_{f}$ and $E_{n}$ are the energies of the states. The presence of the Dirac delta function guarantees overall energy conservation.

Equation (2.1) is derived on three assumptions: that no one-phot in resonances occur; that the two-photon absorption is weak; and that the optical radiation interacts with the quantum system for a time that is long compared to $\hbar /\left(E_{f^{\prime}}-E_{i^{\prime}}-2 \hbar \omega\right)$, where $f^{\prime}$ and $i^{\prime}$ are two arbitrary quantum states such that $E_{f^{\prime}}-E_{i^{\prime}} \neq 2 \hbar \omega$. Also, only electronic transitions are considered; nuclear motions (phonons) are assumed unimportant in the interaction Hamiltonian. Consequently, Eq. (2.1) applies only to direct transitions ( $\Delta \vec{k}=0$ in solids). Indirect transitions will not be considered in this work. They have been treated elsewhere by Yee. ${ }^{22}$

The interaction Hamiltonian of an electromagnetic field with an electron is ${ }^{23}$

$$
H_{i n t}=\frac{e \overrightarrow{\mathbf{A}}(t) \cdot \vec{p}}{m c}+\frac{e^{2} \overrightarrow{\mathbf{A}}(t) \cdot \overrightarrow{\mathbf{A}}(t)}{2 m c^{2}}
$$

where the vector potential $\overrightarrow{\mathbf{A}}(t)$ is given by

$$
\overrightarrow{\mathbf{A}}(t)=A(\vec{r}) \hat{\alpha} e^{-i \omega t}+\text { c.c. }
$$

and where $-e, m, c$ and $\vec{p}$ are respectively the electronic charge, the electronic rest mass, the speed of light in vacuo, and the canonical momentum operator, $-i \hbar \vec{\nabla}$. 
The unit vector $\hat{\alpha}$ specifies the direction of polarization. We adopt the convention thoughout this work that bold-faced vectors represent explicit functions of time. In Eq. (2.2), the transverse (Coulomb) guage is assumed to hold. That is,

$$
\vec{\nabla} \cdot \overrightarrow{\mathbf{A}}(t)=0 .
$$

The optical intensity, I, is given by the time-averaged magnitude of Poynting's vector,

$$
\begin{aligned}
I & =\frac{c n}{4 \pi}\left(\frac{\omega}{c}\right)^{2}[\overrightarrow{\mathbf{A}}(t) \cdot \overrightarrow{\mathbf{A}}(t)]_{a v g} \\
& =\frac{n \omega^{2}}{2 \pi c} A^{2}(\vec{r}) .
\end{aligned}
$$

In the electric dipole approximation, the spatial variation of $A(\vec{r})$ is neglected over atomic dimensions. Matrix elements $[A(\vec{r}) \cdot A(\vec{r})]_{i}$ therefore approximately vanish by orthogonality of wavefunctions for $\mathrm{i} \neq \mathrm{j}$. Since the $[A(\vec{r}) \cdot A(\vec{r})]_{i j}$ terms con̈ribute to two-photon absorption only via first-order perturbation theory (where the initial and final states must be different for a transition to occur), these terms do not contribute to two-photon absorption at all under the electric dipole approximation. This leads to the following expression for the transition matrix elements $H_{i j}^{\prime}$ :

$$
\begin{aligned}
H_{i j}^{\prime} & =\frac{e A(\vec{r})}{m c}[\hat{\alpha} \cdot \vec{p}]_{i j} \\
& =\left|\left(\frac{e}{m \omega}\right)^{2} \frac{2 \pi}{n c} I\right|^{\frac{1}{2}}[\hat{\alpha} \cdot \vec{p}]_{i j}
\end{aligned}
$$

Inserting Eq. (2.6) into Eq. (2.1) gives

$$
W^{(2)}=\frac{2 \pi}{\hbar}\left(\frac{e}{m \omega}\right)^{4}\left(\frac{2 \pi}{n c}\right)^{2} I^{2} \sum_{f} \sum_{i}\left|M_{f i}\right|^{2} \times \delta\left(E_{f}-E_{i}-2 \hbar \omega\right),
$$

where

$$
M_{f i}=\sum_{n} \frac{[\hat{\alpha} \cdot \vec{p}]_{f n}[\hat{\alpha} \cdot \vec{p}]_{n i}}{E_{n}-E_{i}-\hbar \omega}
$$

Starting from Eqs. (2.7)-(2.8) many workers have computed the two-photon absorption coefficient $\beta$ in a variety of crystals. ${ }^{8-13}$ The total rate of energy transferred per unit volume $V$ of material due to two-photon absorption is $2 \hbar \omega W^{(2)} / V$ 
for a uniformly intense beam. This rate of energy transfer equals the rate of energy lost by the beam in propagating a unit distance $\Delta z, \beta I^{2}=-I \partial I / \partial z$. The following relationship for $\beta$ results:

$$
\beta=\frac{2 \hbar \omega W^{(2)}}{I^{2} V}=\sum_{i} \sum_{f} \beta_{f i}
$$

where

$$
\beta_{f i}=2 \omega\left(\frac{e}{m \omega}\right)^{4}\left(\frac{1}{n c}\right)^{2} \frac{(2 \pi)^{3}}{V}\left|M_{f i}\right|^{2} \times \delta\left(E_{f}-E_{i}-2 \hbar \omega\right) .
$$

Equations (2.9) and (2.10) are the central result of time-dependent-perturbation theory for calculating the monochromatic two-photon absorption coeffic ient of solids. They show that the total two-photon absorption coefficient $\beta$ is a sum of two-photon absorption coefficients $\beta_{f i}$ between all initial states $i$ and final states $f$, with each $\beta_{f i}$ given by Eq. (2.10).

\subsubsection{Intermediate States.}

The effective matrix element $M_{f i}$ involves a summation over every state $\mathrm{n}$, as shown by Eq. (2.8). Therefore, one interpretation of Eqs. (2.8)-(2.10) is that two-photon absorption is a summation of two-step processes $i \rightarrow n, n \rightarrow f$, averaged quantum statistically over all states $i, f$, and $n$. The states $n$ are sometimes referred to as "intermediate states." In the independent electron approximation, they may be any of the stationary states of the unperturbed material system. In systems with spatial inversion symmetry, the dipole moment $[\hat{\alpha} \cdot \vec{p}]_{n n}$ vanishes by symmetry. In such systems, one expects that the set of intermediate states will not include either $\mathrm{i}$ or $\mathrm{f}$. However, in materials that are not symmetric with respect to inversion, the initial and final states may also serve as intermediate states.

The concept of an intermediate "virtual" state can easily be misinterpreted. First, the word "state" may seem to imply that the material quantum system is well described through-out the optical transition by a set of eigenenergies. This 
is not so, of course. The external optical field mixes the various quanturn modes of the isolated material. Such an admixture of states is not stationary, which is why one resorts to time-dependent perturbation theory in the first place. ${ }^{23}$ The states $\mathrm{n}$ are not truly intermediate "states." They are merely components of the total admixture of one-electon, pure states that comprise the actual intermediate condition of the system. However, since they are referred to as "intermediate states" in the literature, we shall refer to them in the same way here. When we mean the admixture of all $\mathrm{n}$, we shall denote it in the singular, "the Intermediate State."

The dominant pure eigenmodes of the Intermediate State need not lie energetically between the initial and final states. Pure states with energies above $E_{f}$ and below $E_{i}$ can contribute to two-photon absorption. Figures 2-1(a-e) depict various paths by which two-photon absorption can occur in a solid. The energies shown represent pure states - i.e., states of the unperturbed system. Figure 2-1(f) shows a way that two-photon absorption is often represented. $\mathbf{A}$ dashed line representing a virtual energy level appears midway between the initial and final states. There is no real, unperturbed energy level at the position of the dashed line.

Figure 2-1(a) is particularly interesting, because it appears to represent a process that violates Pauli's Exclusion Principle. In the figure, an electron is shown moving from an occupied orbital of the initial (ground) state of a solid to a lower level $\mathrm{n}$ and then up to a final level $\mathrm{f}$. However, in the ground state, no lower levels are open, so how can the electron pass through a lower orbital? The answer is, it does not. The apparent violation of the Exclusion Principle is an artifact of the model that implicitly underlies Figure 2-1, namely the distinguishable-electron picture. The distinguishable, one-electron picture neglects the presence of all but one electron in a material system. In this picture, it is imagined that an observer can always tell which orbital the electron occupies at any instant. Every orbital is 


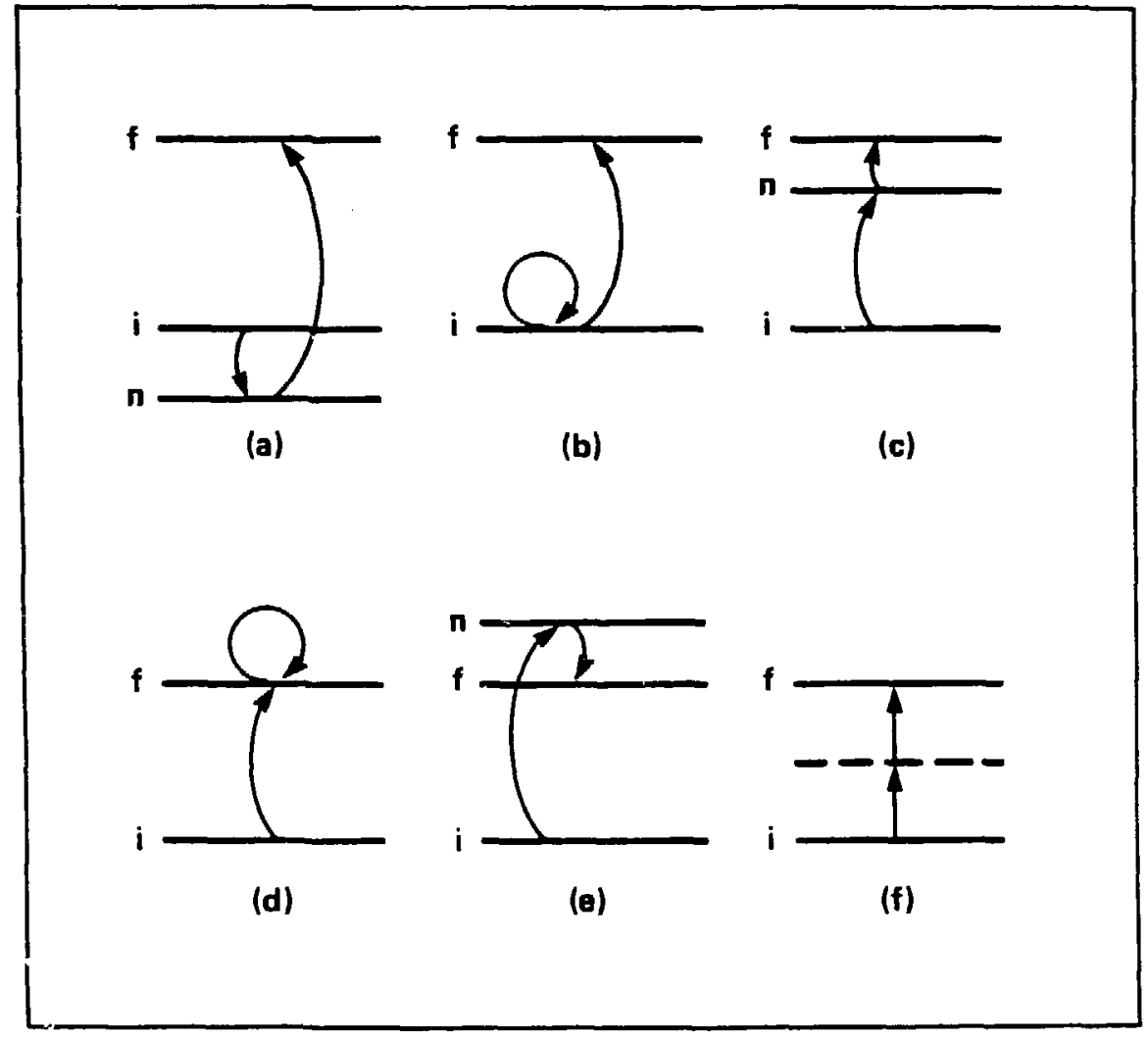

Figure 2-1. Paths by which Two-Photon Absorption Can Occur. (a)-(e): The pure states that can contribute to the intermediate state during two-photon absorption may be any eigenstate in the independent, distinguishable electron model. (f): The composite contribution of all pure states to the intermediate state of the material system is often represented by a virtual energy level, shown by a dashed line. Note that the material system does not behave as if it had an energy halfway between the initial state $\mathrm{i}$ and the final statef. (See text.) 
empty except the one that contains the electron under consideration. It is imagined that the electron is free to move from its initial state to any other state temporarily. The Exclusion Principle is then invoked ad hoc. The presence of other electrons is acknowledged in the initial and final states only, and the original electron is not allowed to remain in an orbital that is occupied by another electron. Thus, it may appear that during two-photon absorption an electron moves from one orbital thro'sh an occupied orbital to an empty orbital, seemingly violating the Exclusion Principle as it passes through the intermediate state.

If instead of a distinguishable-electron picture one uses an indistinguishableelectron picture then it is easy to see that the intermediate state does not violate the Exclusion Principle. In this picture, one describes every electronic state as a Slater determinant of convenient functions. The resultant wave functions are antisymmetric upon exchange of two similar electrons, and so even the Intermediate State rigorously satisfies the Exclusion Principle. (The most accurate statement of the Exclusion Principle is that the total wavefunction of a system of fermions must be antisymmetric when any two similar fermions are exchanged. ${ }^{23}$ ) A clear correspondence between the distinguishable- and the indistinguishable-electron pictures is obtained by choosing distinguishable-electron orbitals as the basis functions in the expansion for the Slater determinant.

Figure 2-2 illustrates the equivalence between the distinguishable- and the indistinguishable-electron pictures. The upper block, A, shows the distinguishableelectron picture of an electron as it passes sequentially from an orbital $i$ in the Ground State through a lower orbital $n$ in the Intermediate State to a final orbital $f$ in the Final State, as in Figure 2-1(a). The lower block, B, shows how the process is modeled in the indistinguishable-electron picture. Initially, the system is in a Ground State with electrons occupying all orbitals up to a certain level. The total 


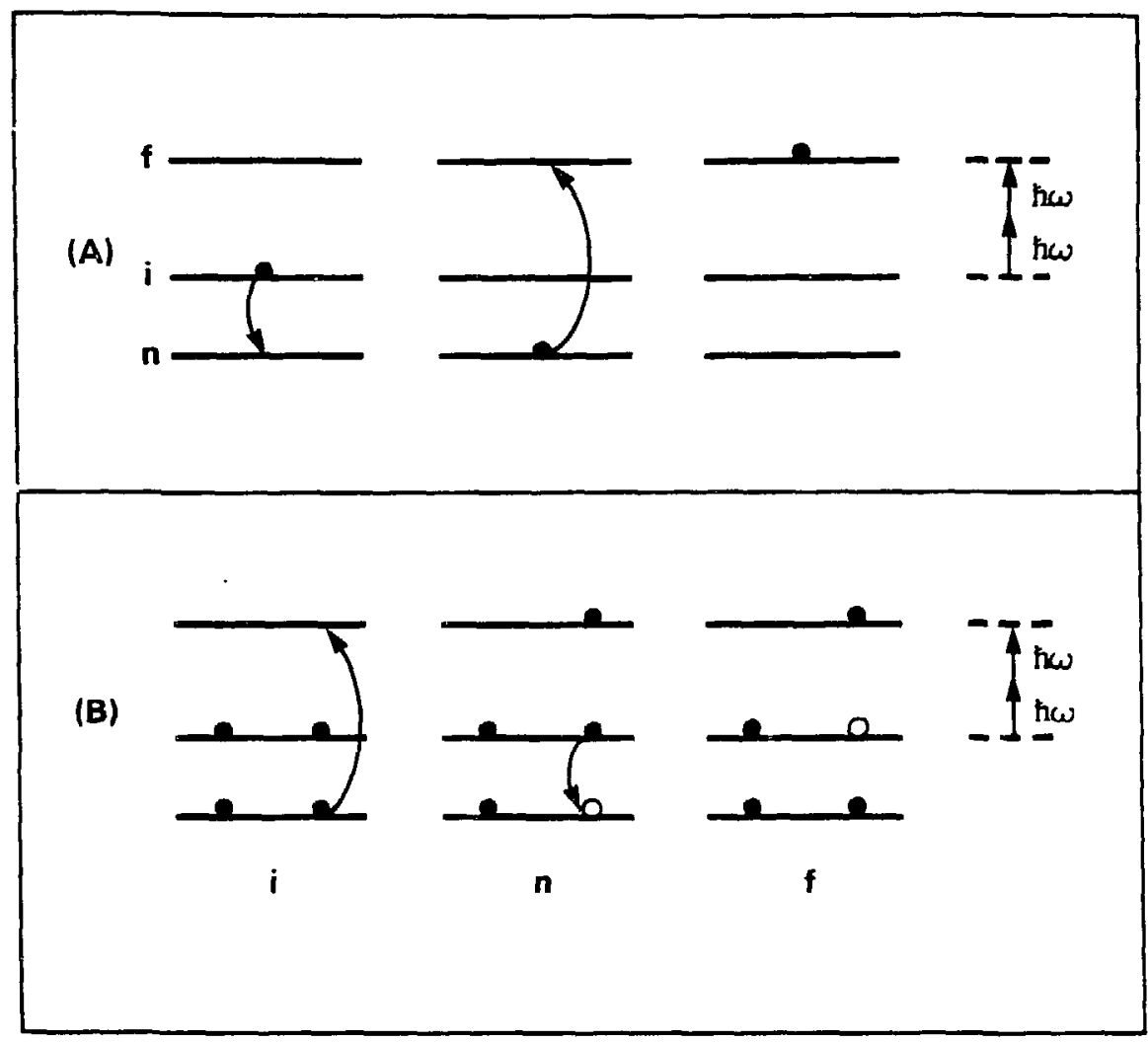

Figure 2-2. Equivalence of $(A)$ the Distinguishable- and (B) the Indistinguishable-Electron Pictures. The states $\mathrm{i}, \mathrm{n}$, and $\mathrm{f}$ are the initial, intermediate, and final states, respectively. In $(A)$, an individual electron appears to move to a lower state and then to a final, higher state. In (B), one electron first evacuates the lower level and then a second electron fills in the hole that is left behind. (A) is formally equivalent to $(B)$. 
Initial State of the system-not just a specific orbital-is called "i." In the Intermediate State, an electron from a low-lying orbital is promoted to an unocupied higher orbital. Then another electron fills in the hole left by the first. Due to the possibility that electrons can exchange orbitals without altering the state of the complete system, there is no way to identify which electron is promoted. The mathematical equivalence between $A$ and $B$ is rigorously exact. The conclusion is that although the process depicted in Figure 2-1(a) appears to violate the Exclusion Principle, it does not really do so. A way of justifying this is to say that time-ordering is lost and that electron exchange has been neglected in $\mathbf{A}$.

The distinguishable-electron and indistinguishable-electron models both yield exactly the same expression for two-photon absorption, as they must, since both models are independent-electron approximations. In both cases, the Exclusion Princıple is obeyed, but this is more obvious in the indistinguishable-electron picture.

\subsubsection{The Single-Intermediate-State Model.}

The single-intermediate-state (SIS) model of two-photon absorption assumes that one pure state $n$ dominates all other material contributions to the effective matrix element $M_{f i}$. Physically, this means that other pure states of the system do not mix strongly with $i$ and $f$, and so they are neglected. This section will examine the SIS model of two-photon absorption within the parabolic-band reduced-zone approximation. First, Braunstein's original dual-frequency SIS theory is given. ${ }^{5}$ Then the results are simplified to two-photon absorption in a single, monochromatic beam. Because of the parabolic-band approximation, the theory is valid only for transitions near the center of the Brillouin zone.

The SIS model was the first one that was applied to two-photon absorption solids. ${ }^{5,24,25}$ Braurstein and Braunstein and Ockman assumed that the only important intermediate states were in the second-lowest conduction band. They chose the 
initial states $i$ from the highest valence band and the final states $f$ from the lowest conduction band. Braunstein implicitly disallowed self-action terms like $\mathrm{i} \rightarrow \mathrm{i}$ and $f \rightarrow f$. He gave ne reason but seemed to suggest that symmetry disallowed them. To estimate the dispersion of the energy bands and the values of the matrix elements, Braunstein used an isotropic effective-mass approximation. Despite the oversimplification, Braunstein's basic approach is today stili the way that intrinsic two-photon absorption coefficients are calculated for direct-gap solids, except that the models used to determine $M_{f i}$ are more detailed and multiple energy bands are used in the summation over intermediate states.

Braunstein ${ }^{5}$ and Braunstein and Ockman ${ }^{24}$ treat two fields at frequencies $\omega_{1}$. and $\omega_{2}$ instead of a single monochromatic field as in Section 2.1. The photon energies $\hbar \omega_{1}$ and $\hbar \omega_{2}$ are such that $\hbar \omega_{1}+\hbar \omega_{2}$ is greater than $E_{G}$ but $\hbar \omega_{1}$ and $\hbar \omega_{2}$ are individually less than $E_{G}$, where $E_{G}$ denotes the band-gap energy. When two frequencies contribute simultaneously to two-photon transitions, Eq. (2.1) becomes

$$
\begin{aligned}
W^{(2)}=\frac{2 \pi}{\hbar} \sum_{f} \sum_{i} & \left|\sum_{n}\left(\frac{H_{f n}^{\prime}{ }^{(2)} H_{n i}^{\prime}{ }^{(1)}}{E_{n}-E_{i}-\hbar \omega_{1}}+\frac{{H_{f n}^{\prime}}^{(2)} H_{n i}^{\prime(1)}}{E_{n}-E_{i}-\hbar \omega_{2}}\right)\right|^{2} \\
& \times \delta\left(E_{f}-E_{i}-\hbar\left(\omega_{1}+\omega_{2}\right)\right),
\end{aligned}
$$

where ${H^{\prime}}^{(s)}$ is the transition matrix element for a beam of frequency $\omega_{s}$, of polarization $\hat{\alpha}^{(s)}$, and of intensity $I_{s}$ (cf. Eq. (2.6)). If the intensity $I_{1}$ is much greater than the intensity $I_{2}$, then it is meaningful to regard the two-photon transition as an absorption of a photon $\hbar \omega_{2}$ that is caused by the intense field at $\omega_{1}$ and to define the induced absorption coefficient at $\omega_{2}$ per unit photon flux $N_{1} c / n_{1}$ as $K_{1}$ $\mathrm{cm}^{-1}$, where $N_{1}$ is the number of photons at $\omega_{1}$ per unit volume and $n_{1}$ is the corresponding refractive index. That is,

$$
K_{1}=\frac{W^{(2)}}{V} \frac{n_{1}}{c N_{1}}
$$


The intensity $\mathrm{I}_{s}$ at a frequency $\omega_{s}$ is given in terms of $N_{s}$ by

$$
I_{s}=\frac{N_{s} c}{n_{s}} \hbar \omega_{s}
$$

A Bloch orbital is indexed by two quantum labels, namely the electronic wave vector and the energy band. "N'e now index the initial (valence) band with a "v," the final (conduction) band with a "c," the intermediate (higher conduction) band with a " $c$ '," and the electronic wave vector by " $\vec{k} . "$ Since the magnitude of an optical wave vector is generally small with respect to the electronic wave vector, $\vec{k}$ is "conserved" in a direct optical transition. Therefore, one may write Eq. as

$$
K_{1}=2 \frac{(2 \pi)^{3}}{\hbar V}\left(\frac{e}{m}\right)^{4} \frac{\hbar}{n_{1} \omega_{1} c} \frac{\hbar N_{2}}{n_{2}^{2} \omega_{2}} \sum_{c} \sum_{v}\left(\sum_{\bar{k}}\left|M_{c v}\right|^{2}\right) .
$$

The leading factor of 2 accounts for spin in this simplified model. The summations over the bands $\mathrm{v}$ and $\mathrm{c}$ have been explicitly included to generalize the model, even though in Braunstein's model only one valence band and one conduction band are used. By analogy with Eq. (2.8), $M_{c v}$ is defined as

$$
M_{c v}=\sum_{c^{\prime}}\left(\frac{\left[\alpha^{(2)} \cdot \vec{p}\right]_{c c^{\prime}}\left[\alpha^{(1)} \cdot \vec{p}\right]_{c^{\prime} v}}{E_{c^{\prime}}-E_{v}-\hbar \omega_{1}}+\frac{\left[\alpha^{(1)} \cdot \vec{p}\right]_{c c^{\prime}}\left[\alpha^{(2)} \cdot \vec{p}\right]_{c^{\prime} v}}{E_{c^{\prime}}-E_{v}-\hbar \omega_{2}}\right) \text {. }
$$

Again for generalization, a summation over $c^{\prime}$ has been used, although the singleintermediate-state model assumes that only one intermediate band contributes to two-photon absorption.

Equations (2.14) and (2.15) can be simplified. First, we assume isotropic, parabolic bands, writing the energies of bands $v, c$, and $c^{\prime}$ as follows:

$$
\begin{aligned}
& E_{v}=-\frac{\hbar^{2} k^{2}}{2 m_{v}} \\
& E_{c}=E_{G}+\frac{\hbar^{2} k^{2}}{2 m_{c}} \\
& E_{c^{\prime}}=E_{c^{\prime}}(0)+\frac{\hbar^{2} k^{2}}{2 m_{c^{\prime}}}
\end{aligned}
$$




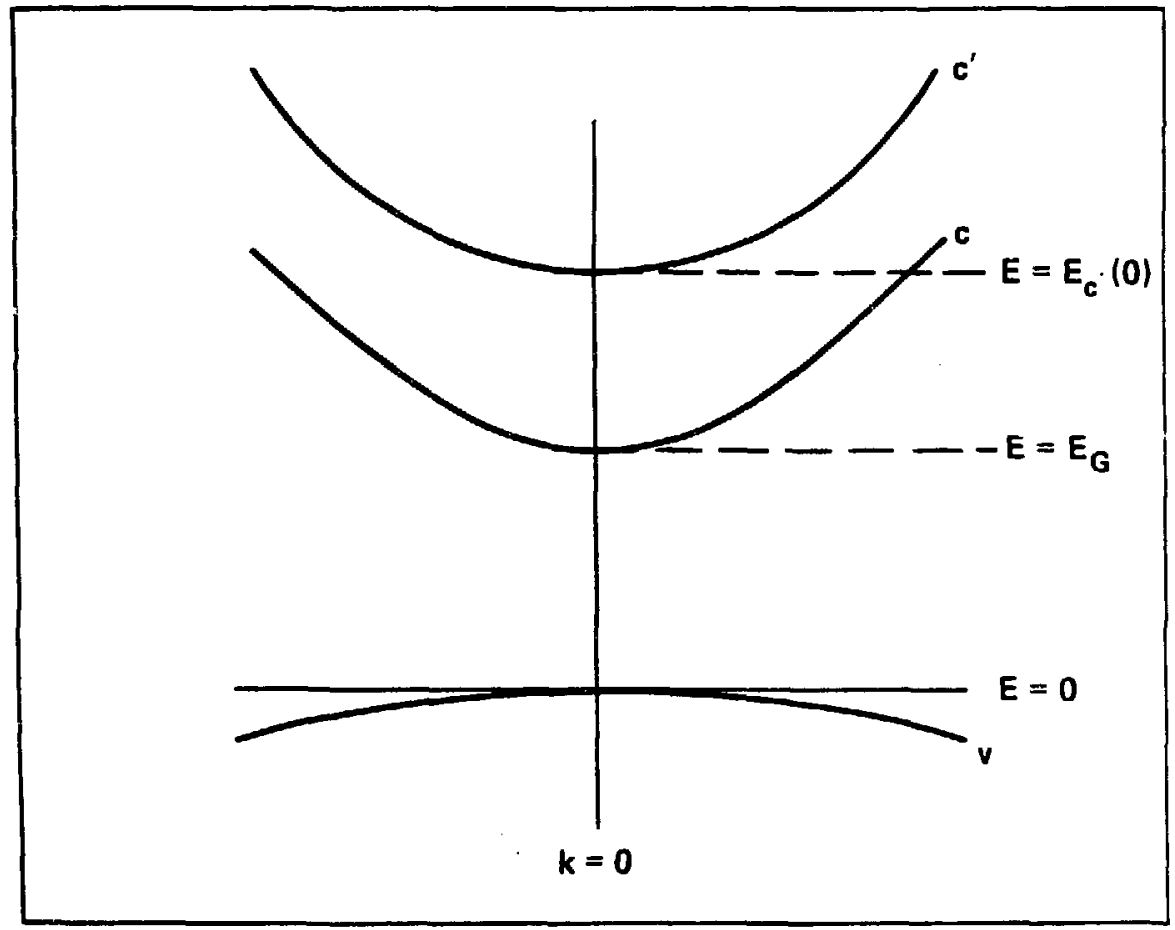

Figure 2-3. Energy Levels in Braunstein's SIS Model. Bands v, $c$, and $c^{\prime}$ are the initial valence, final conduction, and intermediate conduction bands. $E_{G}$ is the band-gap energy. 
Here $E_{c^{\prime}}(0)$ is the diference in energies between the bands $c^{\prime}$ and $v$ at $k=0$, and $m_{v}, m_{c}$, and $m_{c^{\prime}}$ are the effective masses of the various bands. The signs have been chosen so that the effective masses are positive. (See Figure 2-3.) Next, we treat the distribution of wave vectors $\vec{k}$ as quasi-continuous, making the usual transformation ${ }^{26}$

$$
\frac{V}{(2 \pi)^{3}} \int_{B Z} d^{3} \vec{k} \cdots \leftarrow \sum_{\vec{k}} \cdots
$$

From Eq. (2.16), we can transform the above integral from an integral over $\vec{k}$ into an integral over $E_{c v}$, where $E_{c v}$ is defined as the energy difference $E_{c}-E_{v}$. Finally, we simplify the matrix elements $[\hat{\alpha} \cdot \vec{p}]_{i j}$. If $i=j$, then from the equation for the group velocity of an electron onc has ${ }^{26}$

$$
[\hat{\alpha} \cdot \vec{p}]_{j j}=\frac{m}{\hbar} \hat{\alpha} \cdot \vec{\nabla}_{\vec{k}}\left(E_{j}(\vec{k})\right)
$$

From Eqs. (2.16), Eq. (2.18) reduces to

$$
[\hat{\boldsymbol{\alpha}} \vec{p}]_{j j}= \pm \frac{m}{m_{j}} \hbar \hat{\alpha} \cdot \vec{k}
$$

where $m_{j}$ is the effective mass of an electron in band $\mathbf{j}$. The leading sign in Eq. (2.19) is positive for bands with upward curvature and negative for bands with downward curvature. Equation (2.19) is of the same form as the effective transition moment for a dipole-forbidden transition. ${ }^{24}$ The latter is

$$
[\hat{\alpha} \cdot \vec{p}]_{i j}=\frac{m}{m_{i j}^{* *}} \hbar \hat{\alpha} \cdot \vec{k}, \quad \text { (Dipole-forbidden) }
$$

where $m_{i j}^{* *}$ is an effective mass of an electron that undergoes the transition $i \rightarrow j$. For dipole-allowed transitions, the matrix element is independent of $\vec{k}$ to lowest order. We denote this constant by $P_{i j}$ :

$$
\left.P_{i j}=[\hat{\alpha} \cdot \vec{p}]_{i j} . \quad \text { (Dipole }- \text { allowed }\right)
$$


For simplicity, the polarization vectors of the incident fields have been chosen to be identical, $\hat{\alpha}^{(1)}=\hat{\alpha}^{(2)}=\hat{\alpha}$.

Incorporating Eqs. (2.16)-(2.21) into Eq. (2.14) and invoking the SIS approximation gives the following expressions for the induced absorption coefficient $K_{1}$ : Allowed-Allowed :

$$
\begin{aligned}
K_{1} & =\frac{2^{7 / 2} \pi e^{4}}{\hbar^{2} m^{4}} \frac{m_{c v}^{3 / 2}}{n_{1} n_{2} \omega_{1} \omega_{2} c^{2}}\left(\frac{N_{2} c}{n_{2}}\right) \\
& \times\left[\hbar \omega_{1}+\hbar \omega_{2}-E_{G}\right]^{1 / 2}\left|M_{c v}^{\prime \prime}\right|^{2} P_{c c^{\prime}}^{2} P_{c^{\prime} v}^{2}
\end{aligned}
$$

sillowed - Forbidden :

$$
\begin{aligned}
K_{1} & =\frac{2^{9 / 2} \pi e^{4}}{3 \hbar^{2} m^{2} m_{c c^{\prime}}^{* * 2}} \frac{m_{c v}^{5 / 2}}{n_{1} n_{2} \omega_{1} \omega_{2} c^{2}}\left(\frac{N_{2} c}{n_{2}}\right) \\
& \times\left[\hbar \omega_{1}+\hbar \omega_{2}-E_{G}\right]^{3 / 2}\left|M_{c v}^{\prime \prime}\right|^{2} P_{c^{\prime} v}^{2}
\end{aligned}
$$

Forbidden - Allowed :

$$
\begin{aligned}
K_{1} & =\frac{2^{9 / 2} \pi e^{4}}{3 \hbar^{2} m^{2} m_{c^{\prime} v}^{* 2}} \frac{m_{c v}^{5 / 2}}{n_{1} n_{2} \omega_{1} \omega_{2} c^{2}}\left(\frac{N_{2} c}{n_{2}}\right) \\
& \times\left[\hbar \omega_{1}+\hbar \omega_{2}-E_{G}\right]^{3 / 2}\left|M_{c v}^{\prime \prime}\right|^{2} P_{c c^{\prime}}^{2}
\end{aligned}
$$

Forbidden - Forbidden :

$$
\begin{aligned}
K_{1} & =\frac{2^{11 / 2} \pi e^{4}}{5 \hbar^{2} m_{c c^{\prime}}^{*+2} m_{c^{\prime} v}^{*+2}} \frac{m_{c v}^{7 / 2}}{n_{1} n_{2} \omega_{1} \omega_{2} c^{2}}\left(\frac{N_{2} c}{n_{2}}\right) \\
& \times\left[\hbar \omega_{1}+\hbar \omega_{2}-E_{G}\right]^{5 / 2}\left|M_{c v}^{\prime \prime}\right|^{2}
\end{aligned}
$$

The term $M_{c v}^{\prime \prime}$ is the sum of the energy denominators of $M_{c v}$,

$$
M_{c v}^{\prime \prime}=\sum_{s=1,2} \frac{1}{E_{n v}(0)+\left(m_{c v} / m_{c^{\prime} v}\right)\left(\hbar \omega_{1}+\hbar \omega_{2}-E_{G}\right)-\hbar \omega_{s}} .
$$

The quantity $m_{c v}$ is a reduced effective mass defined by

$$
\frac{1}{m_{c v}}=\frac{1}{m_{c}}+\frac{1}{m_{v}}
$$

$m_{c^{\prime} v}$ is defined similarly. The factors of 3 and 5 in the denominators of Eqs. (2.22) are the result of averaging $\cos ^{2} \theta$ and $\cos ^{4} \theta$, respectively, over all angles (0 to $\left.\pi\right)$ in 
the integral over the Brillouin zone. The angle $\theta$ represents the angle between the common polarization vector of the incident fields, $\hat{\alpha}$, and the Bloch wave vector, $\vec{k}$ (cf. Eq. $(2.20))$.

The notations in Eqs. (2.22) show the allowed-ness of each of the successive steps involved in the two-photon transitions. Within the SIS model, we see that the spherical-band approximation leads to two-photon absorption coefficients that are proportional either to $\left[E_{G}-\hbar \omega_{1}-\hbar \omega_{2}\right]^{1 / 2}$ for allowed-allowed transitions, to $\left[E_{G}-\hbar \omega_{1}-\hbar \omega_{2}\right]^{3 / 2}$ for allowed-forbidden transitions, or to $\left[E_{G}-\hbar \omega_{1}-\hbar \omega_{2}\right]^{5 / 2}$ for forbidden-forbidden transitions.

Equations (2.22) correct Braunstein's results for a missing factor of $1 / n_{1} n_{2} .{ }^{12}$ Also, our term $\left|M_{c v}\right|^{2}$ contains cross-products of the two different frequency terms comprising $M_{c v}$ (Eq. (2.15)). Braunstein assumed that the cross-products would vanish due to uncorrelated phase fluctuations of the two incident beams. However, all temporal dependence, including phase changes, is already lumped into the delta function $\delta\left(E_{G}-\hbar \omega_{1}-\hbar \omega_{2}\right)$. There is no general justification for neglecting the cross-products in $\left|M_{c v}\right|^{2}$. Since in this work, we shall be concerned only with monochromatic incident fields, the issue is moot. For monochromatic fields, Eqs. (2.22) are equivalent to the following expressions for two-photon absorption coefficients in units of cm-sec/erg (cf. Eq. (2.9)):

$$
\begin{aligned}
& \text { Allowed-Allowed }: \quad \beta=\frac{2^{9 / 2} \pi e^{4}}{\hbar^{2} m^{4}} \frac{m_{c v}^{3 / 2}}{n^{2} \omega^{2} c^{2}} \\
& \times\left[2 \hbar \omega-E_{G}\right]^{1 / 2}\left|M_{c v}^{\prime}\right|^{2} P_{c c^{\prime}}^{2} P_{c^{\prime} v}^{2},
\end{aligned}
$$

Allowed - Forbidden $: \quad \beta=\frac{2^{11 / 2} \pi e^{4}}{3 \hbar^{2} m^{2} m_{c c^{\prime}}^{* 2}} \frac{m_{c v}^{5 / 2}}{n^{2} \omega^{2} c^{2}}$

Forbidden-Allowed $: \quad \beta=\frac{2^{11 / 2} \pi e^{4}}{3 \hbar^{2} m^{2} m_{c^{\prime} v}^{* 2}} \frac{m_{c v}^{5 / 2}}{n^{2} \omega^{2} c^{2}}$

$$
\times\left[2 \hbar \omega-E_{G}\right]^{3 / 2}\left|M_{c v}^{\prime}\right|^{2} P_{c c^{\prime}}^{2}
$$




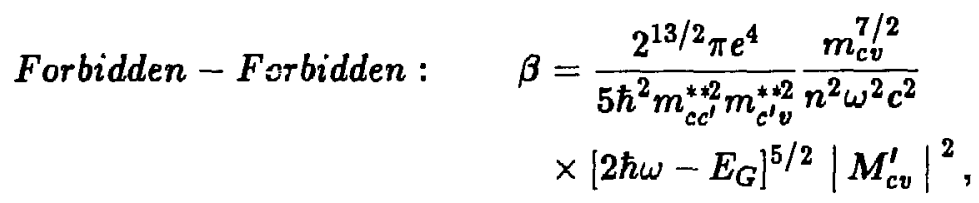

where

$$
M_{c v}^{\prime}=\frac{1}{E_{c^{\prime}}(0)+\left(m_{c v} / m_{c^{\prime} v}\right)\left(2 \hbar \omega-E_{G}\right)-\hbar \omega} .
$$

\subsubsection{Self-interaction Terms.}

Basov et al. ${ }^{6}$ have proposed a three-band model of two-photon absorption that includes self-action (intra-band) terms. Such transitions are depicted in Figures 2-1(b) and 2-1(d). Basov's model includes one conduction band, c, and two valence bands, $v_{1}$ and $v_{2}$, that are degenerate at $k=0$. The bands $v_{1}$ and $v_{2}$ are called the "heavy hole band" and the "light hole band," respectively. ${ }^{27}$ Figure 2-4 shows the band structure near $\mathrm{k}=0$ as Basov et al. have assumed it. This band structure typifies materials with zinc blende compcsition. Basov et al. applied the model to GaAs. In contrast to the model discussed in the previous section, Basov's model disregards conduction bands higher than c. The assumption is that the energy denominators corresponding to levels higher than $c$ or lower than $v_{2}$ are so large that these levels contribute relatively little to the effective transition matrix element $M_{c v}$. Basov and his co-workers also disregard inter-valence-band transitions. The only two possible paths by which a given initial orbital is considered to be excited are, for example, $\left(v_{1} \rightarrow v_{1}, v_{1} \rightarrow c\right)$ and $\left(v_{1} \rightarrow c, c \rightarrow c\right)$. Like Braunstein, Basov uses a spherical-band approximation to estimate both the transition matrix elements $[\hat{\alpha} \cdot \vec{p}]_{i j}$ and the dispersion of the energy bands $E_{i}(\vec{k})$.

The intra-band transitions are dipole-forbidden, and the valence-to-conduction band transitions are dipole-allowed. This is based on the $k=0$ symmetry assignments by Dresselhaus ${ }^{28}$ and Parmenter ${ }^{29}$ for zinc blende materials. Therefore, the effective transition moment $M_{c v}$ in Basov's model is a combination of an allowed- 


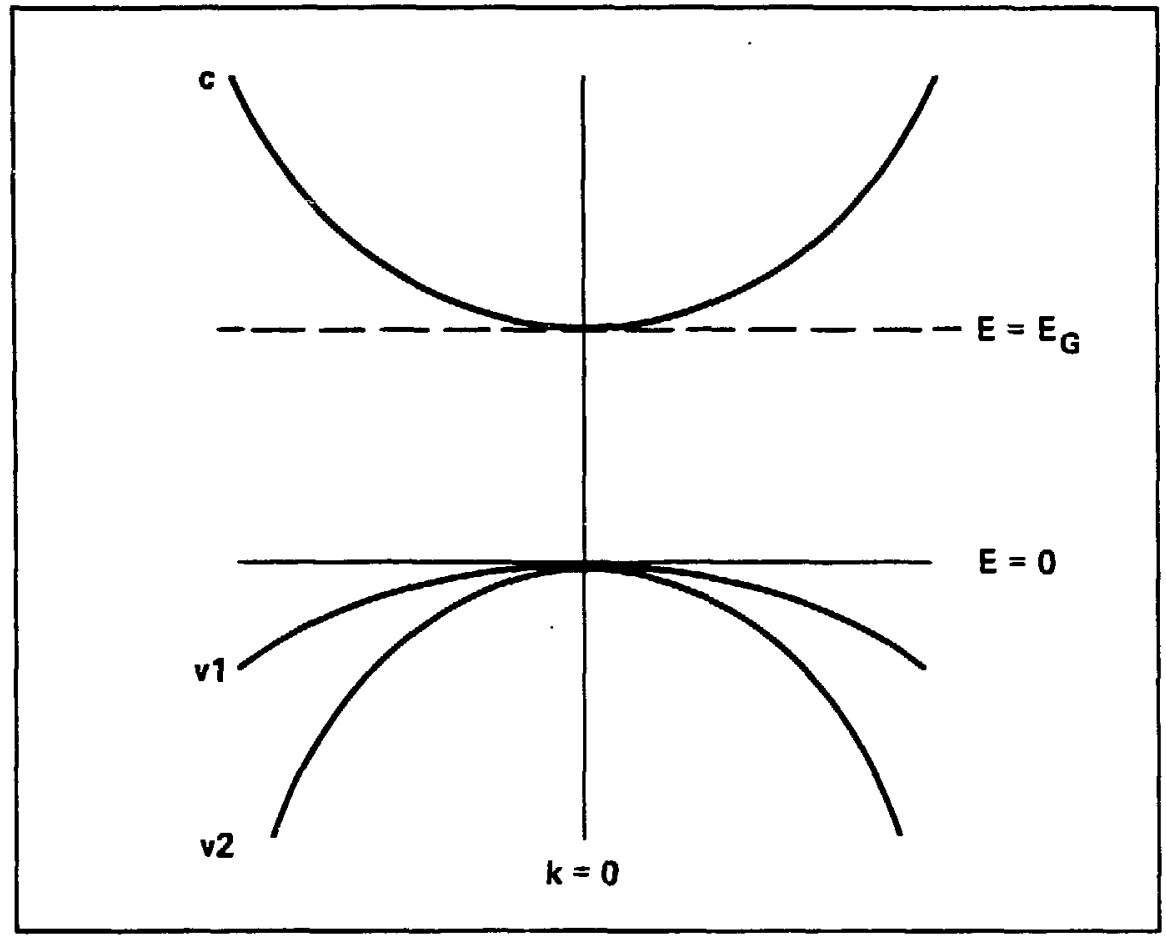

Figure 2-4. Band Structure in Basov's Model. ${ }^{6}$ The model was originally intended for GaAs. Bands v1 and v2 are the heavy- and lighthole bands, respectively. The band $\mathrm{c}$ is the lowest conduction band. 
fortidden transition $(v \rightarrow c, c \rightarrow c)$ and a forbidden-allowed transition $(v \rightarrow v, v \rightarrow c)$. This combination of allowed and forbidden transitions causes Basov's result for $\beta$ to vary as $\left[2 \hbar \omega-E_{G}\right]^{3 / 2}$ near the band edge, as one expects from the discussion in the previous section.

To estimate two-photon absorption cuefficients using Basov's model, one applies Eqs. (2.17)-(2.21) to Eq. (2.10), which is the general expression for $\beta$. This yields

$$
\begin{aligned}
\beta & =2 \sum_{v=v_{1}, v_{2}} \omega\left(\frac{e}{m \omega}\right)^{4}\left(\frac{1}{n c}\right)^{2} \int_{B Z} d \phi \sin \theta d \theta k^{2} d k \\
& \times\left|\frac{\left(m / m_{c}\right) \hbar k \cos \theta P_{c v}}{E_{c}(k)-E_{v}(k)-\hbar \omega}+\frac{P_{c v}\left(-m / m_{v}\right) \hbar k \cos \theta}{-\hbar \omega}\right|^{2} \\
& \times \delta\left(E_{c}(k)-E_{v}(k)-2 \hbar \omega\right) .
\end{aligned}
$$

As before, the leading factor of 2 accounts for the spin degenericy of the initial states. Within the spherical-band approximation of Eq. (2.16), Eq. (2.27) reduces to

$$
\beta=\frac{2^{11 / 2} \pi e^{4}}{3 m^{2}(\hbar \omega)^{5}} \frac{\left[2 \hbar \omega-E_{G}\right]^{3 / 2}}{n^{2} c^{2}} \times \sum_{v=v_{1}, v_{2}} m_{c v}^{1 / 2}\left|P_{c v}\right|^{2} .
$$

Equation (2.28) is Basov's result corrected by a factor of $1 / 3$ for the angular average of $\cos ^{2} \theta$ and by a factor of $1 / 16$ for an error in Basov's definition of intensity. (Basov defined his field amplitude $A(\vec{r})$ the same way we did, but he defined intensity as $n \omega^{2} A^{2} / 8 \pi c$ instead of as $n \omega^{2} A^{2} / 2 \pi c$ as in our Eq. (2.5)). Basov's original result is a factor of 48 too large, as correctly pointed out first by Lee and Fan. ${ }^{30}$

Equation (2.28) predicts an interesting dependence of $\beta$ upon frequency $\omega$. Near the band edge, $\beta$ rises as $\left[2 \hbar \omega-E_{G}\right]^{3 / 2}$, as mentioned. However, before the photon energy reaches $E_{G}, \beta$ reaches an absolute maximum and then falls off asymptotically as $(\hbar \omega)^{-7 / 2}$, neglecting the frequency-dependence of the refractive index, $n$. The maximum value of $\beta$ occurs at $\hbar \omega=5 E_{G} / 7$. The fact that it occurs suggests that in every material an upper limit exists for $\beta$. According to Basov's model, the upper limit scales roughly as $m_{c v}^{1 / 2} E_{G}^{-7 / 2} P_{c v}^{2} / n^{2}$. 
Equations (2.25) and (2.26) from the previous section show that this predicted behavior is not unique to Basov's model. Every type of two-photon transition (allowed-allowed, allowed-forbidden, and forbidden-forbidden) results in a bounded two-photon absorption coefficient as a function of frequency. For the moment, let us disregard the fact that as photon energy increases, one-photon absorption eventually begins. The maximum in $\beta$ is not a manifestation of the finite widths of the initial or final bands. The models so far have assumed infinitely wide bands. (Parabolas are unbounded.) The maximum is not a consequence of direct optical transitions. Indirect transitions would cause $M_{\epsilon v}$ to scale by an extra factor of $k^{4}$, or $\left[2 \hbar \omega-E_{G}\right]^{2}$, at most. This would be insufficient to overcome the factor of $(\hbar \omega)^{5}$ in the denominator of Eq. (2.28), and so $\beta$ would still have a maximum. The maximum in $\beta$ is essentially due to limiting the models so far to a finite number of bands. This guarantees that the density of states will remain finite at all frequencies, and by Fermi's "golden rule" so will $\beta$.

\subsection{Two-photon Absorption in Zinc Blende Compounds.}

Having introduced the perturbation-theory approach and two ways of applying it (Braunstein's and Basow's models), we now consider two-photon absorption for general zinc blende compounds, the significance of which has already been mentioned. The chief refinement of this section is to use wave functions that exhibit the spatial and spin symmetry properties of zinc blende (point group $\overline{43 m}$ ). By so doing, it will be shown that an expression similar to Basov's result ( $\mathrm{Eq}(2.28)$ ) describes two-photon absorption to lowest order in all zinc blende materials. That is, to lovest order, only the initial or final states act as intermediate states. No inter-valence or inter-conduction transitions occur. However, including spin-orbit splitting in the Hamiltonian leads to band splitting and allows intervalence transitions. When the band-gap energy $E_{G}$ is much greater than the spin-orbit splitting 
$\Delta$, the spin-orbit interaction leads to non-parabolic energy band structure. Subsection 2.2.1 examines the effect of this nonparabolicity and finds that it affects the results only weakly for large band-gap materials. Subsection 2.2 .2 considers intervalence- and interconduction-band transitions, following Pidgeon et al. ${ }^{18}$ and Weiler. ${ }^{17}$ As for effects of nonparabolicity, the effects of intervalence transitions are modest. Including intervalence transitions, which are forbidden except for spin effects, raises the maximum theoretical value of $\beta$ only about $40 \%$. Subsection 2.2 .3 concludes the general discussion of zinc blende compounds by discussing excitons. These are important near the band edge, but their relative importance diminishes as the ratio of band-gap energy to excitonic binding energy increases.

To model the eigenfunctions and eigenenergies of zinc blende compounds we use Kane's formalism. This formalism is essentially a four-band model specifically for zinc blende structures. It treats the lowest conduction band, $c$, and the three highest valence bands, v1, v2, and $v 3$, including spin-orbit interactions. Independently Lee and Fan, ${ }^{30}$ Yee $^{31}$ and Fossom and Chang ${ }^{32}$ have proposed models of twophoton absorption using the formalism of $\mathrm{Kane}^{27}$. Except for (1) the addition of a third valence band (the "split-off band") and (2) the use of tro frequencies, their methods essentially resemble Basov's approach. These models, like Braunstein's and Basov's, are valid only for small values of $k$. The higher-order corrections to Kane's orbitals have been ignored, and so the wave functions and energy bands as used by these authors are isotropic. Figure 2-5 depicts the energy band scheme now under consideration.

Kane's dispersion relations for the energy $E$ as a function of the wave vector are given by the four roots to the following equation:*

$$
0=E^{2}\left(E-E_{G}\right)(E+\Delta)-E(E+2 \Delta / 3) \frac{\hbar^{2} k^{2} P^{2}}{m^{2}} .
$$

* In this work, $\mathrm{P}$ is defined differently than in Kane's work: $P_{K a n e}=\hbar P / m$. 


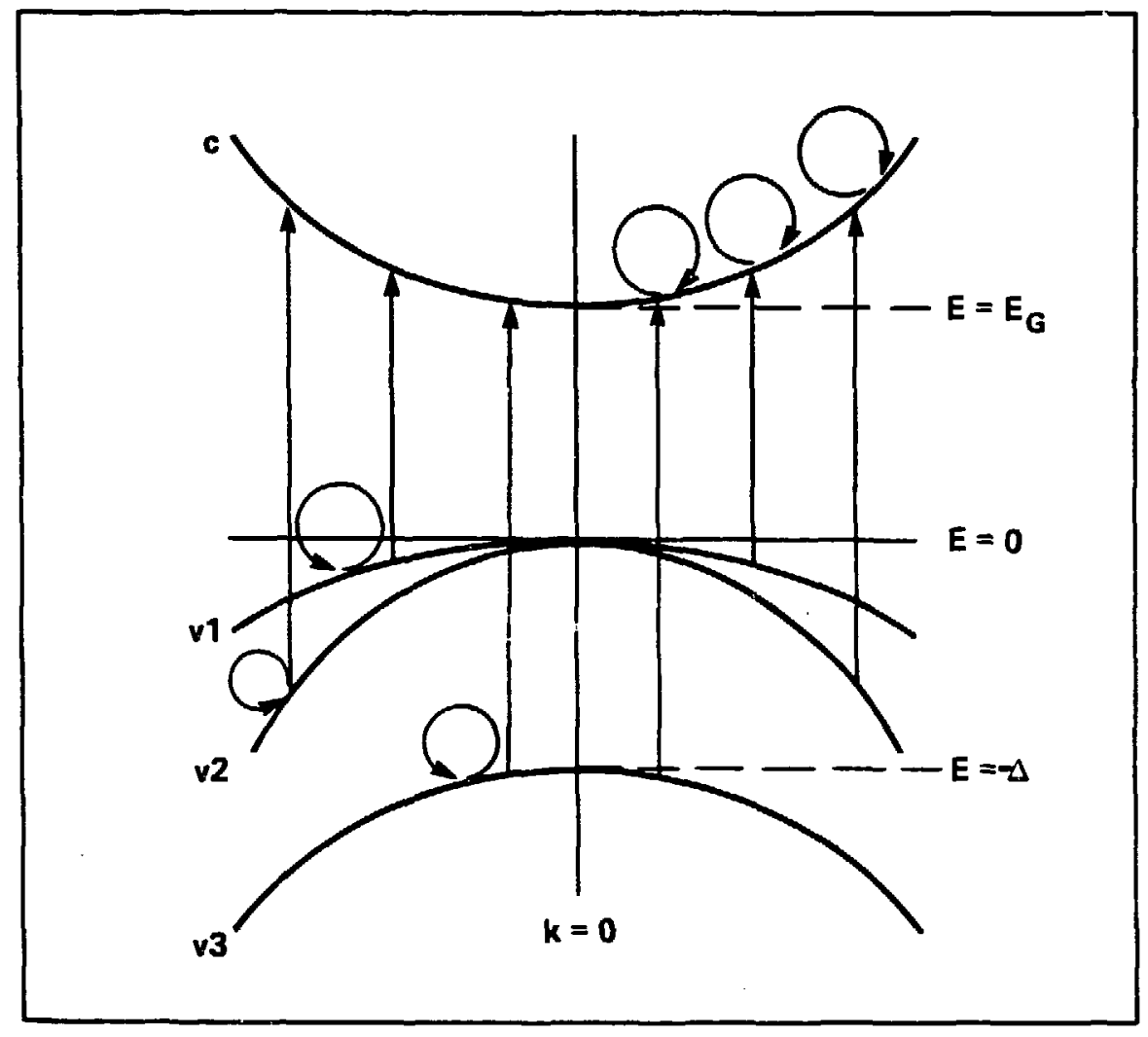

Figure 2-5. Two-Photon Transitions Based on Kane's Band Structure. (See Reference 31, for example.) Intervalence-band transitions are forbidden by symmetry, to first order. 
This equation is the secular equation of the $4 \times 4$ matrix that represents Kane's Hamiltonian operator. Here, as previously, the band-gap energy is denoted by $E_{G}$. The spin-orbit splitting of the split-off band is denoted by $-\Delta, k$ is the magnitude of the Bloch wave vector, and $P$ is a momentum matrix element that will be defined below. Kane has chosen the energies so that for every $k, E$ in Eq. (2.29) represents the energy difference between any band and the heavy-hole band, v1. Thus, one root of Eq. (2.29) is $E=0$. Although Eq. (2.29) is quartic, the four roots are approximately parabolic functions of $k$ for small $k$. In the parabolic approximation, the band energies are given by the following familiar expressions: ${ }^{30,31}$

$$
\begin{aligned}
& E_{c}=E_{G}+\frac{\hbar^{2} k^{2}}{2 m_{c}} \\
& E_{v 1}=-\frac{\hbar^{2} k^{2}}{2 m_{v 1}} \\
& E_{v 2}=-\frac{\hbar^{2} k^{2}}{2 m_{v 2}} \\
& E_{v 3}=-\Delta-\frac{\hbar^{2} k^{2}}{2 m_{v 3}} .
\end{aligned}
$$

With the symmetry assignments of Dresselhaus, ${ }^{28}$ Kane represents the spatial dependence of the four bands with a basis of orthonormal states that are denoted $S, X, Y$, and $Z$. The notation emphasizes that, at $k=0$, the conduction state is essentially s-like and the valence states are essentially p-like. The directions $\hat{x}, \hat{y}$, and $\hat{z}$ are chosen along the principal axes of the material. The spin functions are denoted by arrows $\uparrow$ and $\downarrow$. The orbitals for $k \neq 0$, which are determined from $\vec{k} \cdot \vec{p}$ theory, have the following forms:

$$
\begin{gathered}
\phi_{c \alpha}=i[S \uparrow]^{\prime} \\
\phi_{c \beta}=i[S \downarrow]^{\prime} \\
\phi_{v 1 \alpha}=[(X+i Y) \uparrow]^{\prime} / \sqrt{2} \\
\phi_{v 1 \beta}=[(X+i Y) \downarrow]^{\prime} / \sqrt{2}
\end{gathered}
$$




$$
\begin{aligned}
& \phi_{v 2 \alpha}=[(X-i Y) \uparrow]^{\prime} / \sqrt{6}+[Z \downarrow]^{\prime} \sqrt{2 / 3} \\
& \phi_{v 2 \beta}=[(X-i Y) \downarrow]^{\prime} / \sqrt{6}+[Z \uparrow]^{\prime} \sqrt{2 / 3} \\
& \phi_{v 3 \alpha}=[(X-i Y) \uparrow]^{\prime} / \sqrt{3}-[Z \downarrow]^{\prime} / \sqrt{3} \\
& \phi_{v 3 \beta}=[(X-i Y) \downarrow]^{\prime} / \sqrt{3}-[Z \uparrow]^{\prime} / \sqrt{3}
\end{aligned}
$$

In these expressions, the subscripts $\alpha$ and $\beta$ denote states of opposite spin parity, and the prime means that the basis functions have been transformed according to the relations below:

$$
\begin{gathered}
S^{\prime}=S \\
{\left[\begin{array}{l}
X \\
Y \\
Z
\end{array}\right]^{\prime}=\left[\begin{array}{ccc}
\cos \theta \cos \phi & \cos \theta \sin \phi & \sin \theta \\
-\sin \theta & \cos \theta & 0 \\
\sin \theta \cos \phi & \sin \theta \sin \phi & \cos \theta
\end{array}\right]\left[\begin{array}{l}
X \\
Y \\
Z
\end{array}\right],}
\end{gathered}
$$

and

$$
\left[\begin{array}{l}
\uparrow \\
\downarrow
\end{array}\right]^{\prime}=\left[\begin{array}{cc}
e^{-i \phi / 2} \cos \theta / 2 & e^{i \phi / 2} \sin \theta / 2 \\
-e^{-i \phi / 2} \sin \theta / 2 & e^{i \phi / 2} \cos \theta / 2
\end{array}\right]\left[\begin{array}{l}
\uparrow \\
\downarrow
\end{array}\right] .
$$

In Eqs. (2.32) $\theta$ is the angle between $\vec{k}$ and the z-axis of the crystal, and $\phi$ is the usual azimuthal angle measured from the x-axis.

Now we consider the momentum matrix elements between every pair of orbitals listed in Eqs.(2.31). Let $\phi_{i}$ and $\phi_{j}$ denote two orbitals. First, if $i=j$, then for small $k$ Eq. (2.19) gives

$$
\left\langle\phi_{j}|\vec{p}| \phi_{j}\right\rangle= \pm \frac{m}{2 m_{j}} \hbar \vec{k}
$$

Next, if $i \neq j$, then we may invoke the following symmetry and orthogonality relations in which we now define $P$ :

$$
\begin{gathered}
\left\langle i S\left|p_{x}\right| X\right\rangle=\left\langle i S\left|p_{y}\right| Y\right\rangle=\left\langle i S\left|p_{z}\right| Z\right\rangle=P, \\
\left\langle i S\left|p_{x}\right| Y\right\rangle=\left\langle i S\left|p_{y}\right| X\right\rangle=0 \\
\left\langle i S\left|p_{y}\right| Z\right\rangle=\left\langle i S\left|p_{z}\right| Y\right\rangle=0 \\
\left\langle i S\left|p_{z}\right| X\right\rangle=\left\langle i S\left|p_{x}\right| Z\right\rangle=0 \\
\langle\uparrow \mid \uparrow\rangle=\langle\downarrow \mid \downarrow\rangle=1
\end{gathered}
$$


and

$$
\langle\uparrow \mid \downarrow\rangle=\langle\downarrow \mid \uparrow\rangle=0
$$

From these relations, it follows that the momentum matrix elements between the primed basis states are given by

$$
\begin{aligned}
\left\langle i S^{\prime}|\vec{p}| X^{\prime}\right\rangle & =\left\langle i S|\vec{p}| R_{\mu \nu} X_{\nu}\right\rangle \\
& =R_{\mu \nu} \hat{x}_{\nu} P \\
& =\hat{x}_{\mu}^{\prime} P .
\end{aligned}
$$

In this relation, repeated subscripts imply a summation, and $R_{\mu \nu}$ is an element of the rotation matrix in Eq. (2.32b). The unit vectors $\hat{x}_{\mu}^{\prime}$ and $\hat{x}_{\nu}$ are along the Cartesian axes in the rotated and unrotated frames of reference, respectively. $X_{\mu}^{\prime}$ and $X_{\nu}$ are the corresponding p-like functions. Table 2-1 gives the momentum matrix elements to first order in $k$ for all pairs of Kane's orbitals. The table is taken from Lee and Fan's Table 1, disregarding terms that are of the order $\hbar k P$ or $P^{2}$.

From Table 2-1, one sees that, in zinc blende materials, all intervalence-band transitions and interconduction-band transitions are disallowed to first order. (This is why the models of Yee and Lee and Fan to lowest order resemble that of Basov.) Disregarding intervalence transitions greatly simplifies the sum over intermediate states for each effective transition moment $M_{c v}$. (Here $c$ denotes an arbitrary conduction band, and $v$ denotes an arbitrary valence band.) It is not a general result that intervalence transitions and interconduction-band transitions are forbidden in every material. It is a consequence of symmetry in the zinc blende structure, of the order of the approximation, and of the particular bands under consideration.

The two-photon absorption coefficient is given by Eq. (2.10). Converting the sum over all $k$ into an integral and changing the variable of integration from $k$ to 


\begin{tabular}{|c|c|c|c|c|c|c|c|c|}
\hline & $c \alpha$ & $c \beta$ & v1a & $v 1 \beta$ & $\mathrm{v} 2 \alpha$ & $\mathrm{v} 2 \beta$ & $\mathbf{v 3 \alpha}$ & $\mathbf{v} \boldsymbol{\beta}{ }^{i "}$ \\
\hline $\begin{array}{l}c \alpha \\
c \beta\end{array}$ & $\left(m / m_{r}\right) \hbar \vec{k}$ & $\begin{array}{c}0 \\
\left(m / m_{*}\right) \hbar \vec{k}\end{array}$ & $\begin{array}{c}0 \\
(\hat{x}+i \hat{y})^{\prime} P / \sqrt{2}\end{array}$ & $\begin{array}{c}(\hat{x}+i \hat{y})^{\prime} P / \sqrt{2} \\
0\end{array}$ & $\begin{array}{c}\hat{z}^{\prime} P \sqrt{2 / 3} \\
(\hat{x}-i \hat{y})^{\prime} P / \sqrt{6}\end{array}$ & $\begin{array}{c}-(\hat{x}+i \hat{y})^{\prime} P / \sqrt{6} \\
\hat{z}^{\prime} P \sqrt{2 / 3}\end{array}$ & $\begin{array}{c}-\hat{z}^{\prime} P / \sqrt{3} \\
(\hat{x}+i \hat{y})^{\prime} P / \sqrt{3}\end{array}$ & $\begin{array}{c}-(\hat{x}+i \hat{y})^{\prime} P / \sqrt{3} \\
\hat{z}^{\prime} P / \sqrt{3}\end{array}$ \\
\hline vla & & & $\left(m / m_{11}\right) \hbar \vec{k}$ & 0 & 0 & 0 & 0 & 0 \\
\hline$v 1 \beta$ & & & & $\left(m / m_{n 1}\right) \hbar \vec{k}$ & 0 & 0 & 0 & 0 \\
\hline v $2 \alpha$ & & & & & $\left(m / m_{n 1}\right) \hbar \vec{k}$ & 0 & 0 & 0 \\
\hline$\vee 2 \beta$ & & & & & & $\left(m / m_{11}\right) \hbar \vec{k}$ & 0 & 0 \\
\hline $\mathbf{v} 3 \alpha$ & & & & & & & $\left(m / m_{1,1}\right) \hbar \vec{k}$ & 0 \\
\hline $\mathbf{v} 3 \beta$ & & & & & & & & $\left(m / m_{11}\right) \hbar \vec{k}$ \\
\hline
\end{tabular}

Table 2-1. Lowest-order transition matrix elements $\langle m|\vec{p}| n\rangle$ from Kane's theory in the parabolic-band approximation. (Adapted from Refs. 27 and 30.) 
energy by using Eqs.(2.30), one obtains the following result for $\beta$ :

$$
\begin{gathered}
\beta=\frac{2^{11 / 2} \pi e^{4} P^{2}}{45 n^{2} c^{2} m^{2}}\left(\frac{1}{\hbar \omega}\right)^{5} \\
\times\left\{\begin{array}{c}
{\left[2 \hbar \omega-E_{G}\right]^{3 / 2} \Theta\left(2 \hbar \omega-E_{G}\right)\left(3 \sqrt{m_{c v 1}}+7 \sqrt{m_{c v 2}}\right)} \\
+\left[2 \hbar \omega-E_{G}-\Delta\right]^{3 / 2} \Theta\left(2 \hbar \omega-E_{G}-\Delta\right) 5 \sqrt{m_{c v 3}}
\end{array}\right\}
\end{gathered}
$$

In Eq. (2.35), $\Theta(x)$ is a Heavyside unit step function. It assures that the terms inside the square brackets are non-negative. Physically, it represents a spectral "turning-on" function that shows when various energy bands begin to contribute to two-photon absorption. The frequency dependence of Eq. (2.35) shows that twophoton absorption near the band edge in zinc blende materials is a sum of allowedforbidden processes. Equation (2.35) is valid for linearly polarized, monochromatic light. Because of the isotropic band approximation, $\beta$ is predicted to be independent of angle. In actuality, as we shall discuss later in this chapter, $\beta$ must depend on the polarization, even for isotropic crystals.

\subsubsection{Nonparabolic Energy Bands.}

Kane's dispersion relation, Eq. (2.29), provides a way to treat the nonparabolic behavior of energy bands. Because Kane's formalism is based upon perturbation theory near $k=0$, the range of validity of the model is restricted to small $k$,even for non-parabolic bands. Vaidyanathan et al. ${ }^{12,33-36}$ and Pidgeon et al. ${ }^{18}$ have asserted that the effect of non-parabolic bands should change $\beta$ roughly by a factor of 2 to 7 . Unfortunately, those conclusions were based on modeling errors. When the errors are corrected, using non-parabolic bands affects $\beta$ very little, as this section will show.

When the spin-orbit energy splitting is much greater than the band-gap energy $\left(\Delta \gg E_{G}\right)$ or vice versa, then Kane's quartic dispersion relation reduces to a quadratic equation. The case for large $\Delta$ has been formulated by Pidgeon and co- 
workers, neglecting the split-off band. ${ }^{18} \mathrm{~A}$ sign error has been corrected by Weiler. ${ }^{17}$ The case for $\Delta \ll E_{G}$ has not been correctly addressed by anyone.

Since we are primarily interested in large band-gap, zinc blende materials in this work, we shall examine the latter case. In passing, we note that Pidgeon et al. proposed a model for $\beta$ for this case. ${ }^{18}$ However, that model uses a simplification of Kane's dispersion relation that is valid only when $\Delta \gg E_{G}$. Therefore the expression for $\beta$ that Pidgeon and co-workers have given in the limit of small $\Delta$ is not applicable to large-band-gap zinc blende materials.

We begin by rewriting Kane's dispersion relation as follows:

$$
0=-E^{\prime \prime 3}+E^{\prime \prime 2}\left(1-\frac{\Delta}{E_{G}}\right)+E^{\prime \prime}\left(\frac{\Delta}{E_{G}}+\left(\frac{\hbar k P}{m E_{G}}\right)^{2}\right)-\frac{2 \Delta}{3 E_{G}}\left(\frac{\hbar k P}{m E_{G}}\right)^{2},
$$

where $E^{\prime \prime}$ is the energy $E$ of Eq. (2.29) normalized by the band-gap energy,

$$
E^{\prime \prime}=E / E_{G}
$$

If we consider only the valence bands and restrict attention to small $k$ and small $\Delta$, then $E^{\prime \prime}$ is much less than unity. Therefore, the cubic term in Eq. (2.36) is negligible, and the coefficient of $E^{\prime \prime 2}$ is approximately unity. The resulting quadratic equation is easily solved. The energies of the various bands are given by the following expressions:

where

$$
\begin{aligned}
& E_{c}=\frac{\hbar^{2} k^{2}}{2 m}+E_{G}\left(1+\left(\frac{\hbar k P}{m E_{G}}\right)^{2}\right) \\
& E_{v 1}=\frac{\hbar^{2} k^{2}}{2 m} \\
& E_{v 2}=\frac{\hbar^{2} k^{2}}{2 m}-\frac{E_{G}}{2}\left[\frac{\Delta}{E_{G}}+\left(\frac{\hbar k P}{m E_{G}}\right)^{2}-D_{k}\right] \\
& E_{v 3}=\frac{\hbar^{2} k^{2}}{2 m}-\frac{E_{G}}{2}\left[\frac{\Delta}{E_{G}}+\left(\frac{\hbar k P}{m E_{G}}\right)^{2}+D_{k}\right]
\end{aligned}
$$

$$
D_{k}=\sqrt{\left(\frac{\Delta}{E_{G}}+\left(\frac{\hbar k P}{m E_{G}}\right)^{2}\right)^{2}-\frac{8 \Delta}{3 E_{G}}\left(\frac{\hbar k P}{m E_{G}}\right)^{2}} \text {. }
$$


The correctness of Eqs.(2.38) can be verified by expanding the terms to lowest order in $k^{2}$ and comparing the result to the parabolic approximations given by Kane.

The presence of $\hbar^{2} k^{2} / 2 m$ in each of these equations arises from $\vec{k} \cdot \vec{p}$ perturbation theory. Although Eq. (2.38b) appears to suggest that the valence band $v 1$ has a positive curvature, this may be disregarded. Only differences in energy are physically meaningful, and for direct transitions the term in question vanishes when one considers the difference in energy between two bands.

To find $\beta$, we shall need to solve several integrals of the following form:

$$
\beta_{c v} \approx \int_{0}^{2 \pi} d \phi \int_{-1}^{1} d \cos \theta \int_{k^{2}} \frac{1}{2} k d k^{2} M_{c v}^{2} \delta\left(E_{c}(k)-E_{v}(k)-2 \hbar \omega\right) .
$$

This can be done with the aid of Eqs.(2.38). For a given pair of bands, we first find the interband energy difference $E_{c v}(k)=E_{c}(k)-E_{v}(k)$. Then we invert that expression to find the function $k^{2}\left(E_{c v}\right)$. The results are

$$
\begin{aligned}
k^{2} & =\left(\frac{m E_{G}}{\hbar P}\right)^{2}\left(\frac{E_{c, v 1}}{E_{G}}-1\right) \\
k^{2} & =\left(\frac{m E_{G}}{2 \hbar P}\right)^{2}\left[3\left(\frac{E_{c, v 2}}{E_{G}}-1\right)-\frac{5 \Delta}{3 E_{G}}+D_{E}\right]^{3 / 2} \\
k^{2} & =\left(\frac{m E_{G}}{2 \hbar P}\right)^{2}\left[3\left(\frac{E_{c, v 3}}{E_{G}}-1\right)-\frac{5 \Delta}{3 E_{G}}-D_{E}\right]^{3 / 2}
\end{aligned}
$$

where

$$
D_{E}=\sqrt{\left(\frac{E_{c, v 3}-\Delta}{E_{G}}-1\right)^{2}+\left(\frac{4 \Delta}{3 E_{G}}\right)^{2}} .
$$

As in Section 2.2 we consider only the lowest-order contributions to the momentum matrix elements. Therefore, we again disregard intervalence contributions to $M_{c v}$, and we use Table 2-1 to approximate the momentum matrix elements. To find the intra-band matrix elements, we use Eq. (2.18) and Eqs.(2.38) in Eq. (2.8). The resulting expression for $\beta$ is

$$
\beta=\beta_{c, v 1}+\beta_{c, v 2}+\beta_{c, v 3},
$$


where

$$
\begin{aligned}
\beta_{c, v 1} & =\beta_{0} 3\left(\frac{\hbar \omega}{E_{G}}\right)^{-5}\left(\frac{2 \hbar \omega}{E_{G}}-1\right)^{3 / 2} \Theta\left(2 \hbar \omega-E_{G}\right) \\
\beta_{c, v 2} & =\beta_{0}(7 / 2)\left(\frac{\hbar \omega}{E_{G}}\right)^{-5}\left[3\left(\frac{2 \hbar \omega}{E_{G}}-1\right)-\frac{5 \Delta}{3 E_{G}}+D_{\omega}\right]^{3 / 2} \\
& \times \Theta\left(2 \hbar \omega-E_{G}\right) \div\left[3+\left(\frac{2 \hbar \omega-\Delta}{E_{G}}-1\right) / D_{\omega}\right] \\
\beta_{c, v 3} & =\beta_{0}(5 / 2)\left(\frac{\hbar \omega}{E_{G}}\right)^{-5}\left[3\left(\frac{2 \hbar \omega}{E_{G}}-1\right)-\frac{5 \Delta}{3 E_{G}}-D_{\omega}\right]^{3 / 2} \\
& \times \Theta\left(2 \hbar \omega-E_{G}-\Delta\right) \div\left[3-\left(\frac{2 \hbar \omega-\Delta}{E_{G}}-1\right) / D_{\omega}\right],
\end{aligned}
$$

where

$$
D_{\omega}=\sqrt{\left(\frac{2 \hbar \omega-\Delta}{E_{G}}-1\right)^{2}+\left(\frac{4 \Delta}{3 E_{G}}\right)^{2}} .
$$

In these expressions, $\beta_{0}$ is defined as

$$
\beta_{0} \equiv \frac{2^{5} \pi e^{4} P}{45 E_{G}^{3} m c^{2} n^{2}}
$$

Equations (2.42) and (2.43) show that $\beta$ has a maximum value which, for small $\Delta$, scales basically as $P / E_{G}^{3} n^{2}$. Pidgeon was the first to point out this scaling law for zinc blende materials. ${ }^{18}$ This rule should apply not just to zinc blende compounds but to all materials in which two-photon absorption is due only to allowed-forbidden types of transitions. The broad applicability of this scaling law is important.

In many zinc blende materials, the effective mass of a conduction band electron is much less than the effective masses of the valence electrons. Consequently, the effective reduced masses of the interband transitions are all approximately equal. That is,

$$
m_{c, v 1} \approx m_{c, v 2} \approx m_{c, v 3}
$$

From Eq. (2.38) it follows that

$$
m_{c, v 1}=\frac{m^{2} E_{G}}{2 P^{2}} .
$$


These last two equations provide a way to compare Basov's formula (Eq. (2.28)) to the results of the non-parabolic band, Kane's-function formalism (Eqs. (2.41)(2.43)). The two models are practically identical in the limit of small spin-orbit splitting $\left(\Delta \ll E_{G}\right.$ ), except that the latter model treats the split-off band that Basov neglects.

The final conclusion here is that for large-band-gap zinc blende materials (i.e., those where $\Delta / E_{G}<<1$ ), effects of nonparabolicity in the energy bands may be neglected. This follows from the fact that Eqs. (2.38) are parabolic in $k$ when $\Delta \rightarrow 0$.

\subsubsection{Intervalence- and Interconduction-Band Transitions in Zinc Blende.}

Lee and $\operatorname{Fan}^{30}$ have tabulated the matrix elements $\left[\hat{\alpha}^{\cdot} \vec{p}\right]_{i j}$ between all possible pairs of Kane orbitals in the parabolic-band approximation. Although at $k=0$ the conduction bands are mostly s-like and the valence bands are mostly p-like, away from $k=0$ the s- and p-like functions intermix. Due to the intermixing, several intervalence transitions can contribute to two-photon absorption. Interconductionband transitions, however, still vanish, due to cancelling s-p interactions.

Pidgeon et al. ${ }^{18}$ have used Lee and Fan's parabolic-band matrix elements and Kane's nonparabolic-band formalism in a three-band model of two-photon absorption. The energy band expressions that Pidgeon and co-workers used are valid only in the region where $\Delta \gg E_{G}$, as previously mentioned. (cf. Kane ${ }^{27}$ Eq. (13).) In this region, the split-off valence band does not interact strongly with the other bands, and so Pidgeon et al. ignore it. They obtain an expression for $\beta$ which is similar in form to Basov's results and to Eqs. (2.41) $-(2.43)$ in that $\beta$ is shown to be a scalar (which varies as $P E_{G}^{-3} n^{-2}$ ) times a "universal" function of the dimensionless quantity $\hbar \omega / E_{G}$. According to Pidgeon's estimate, using the nonparabolic dispersion relations increases the theoretical value of $\beta$ by as much as a factor of 
seven. Other authors have attempted to account for nonparabolic bands in an ad hoc fashion in zinc blende materials, ${ }^{34-36}$ but Pidgeon was the first to use the correct (i.e., Kane's) expression for nonparabolic-energy bands for materials with large $\Delta$. There is a slight inconsistency in the latter work in that Pidgeon et al. use nonparabolic-energy bands but they use parabolic-band wave functions to estimate matrix elements.

Weiler ${ }^{17}$ has carefully repeated Pidgeon's calculations, making two changes. First, Weiler has corrected a sign error in Pidgeon's sum over intermediate states. Second, Weiler has used a nonparabolic form for the matrix elements $[\hat{\alpha} \cdot \vec{p}]_{i j}$. (cf. Kane, ${ }^{27}$ Eqs. (13)-(17).) Interestingly, both Weiler and Pidgeon report good agreement between experiment and theory. However, unlike Pidgeon, Weiler shows that using nonparabolic bands decreases the theoretical values of $\beta$ by about $40 \%$ near the maximum value of $\beta(\omega)$ (cf. Weiler ${ }^{17}$, Fig. 1). This result for small band-gap materials agrees with the conclusions of the previous section for large band-gap materials. That is, using nonparabolic bands affects $\beta$ only slightly (i.e., by much less than an order of magnitude.)

Weiler's expression for $\beta$ is

$$
\beta=\frac{4 \pi e^{4} P}{m c^{2} E_{G}^{3} n^{2}} f\left(\hbar \omega / E_{G}\right),
$$

where, in the nonparabolic model,

$$
f^{n p}(x)=\frac{(2 x-1)^{3 / 2}}{3 x^{3}}\left\{\frac{4(3 x)^{1 / 2}}{(3 x-1)^{2}}+\left(3 x+\frac{3}{2}\right)^{3 / 2} \frac{9 x^{4}+10 x^{2}+6}{90 x^{5}}\right\} .
$$

In the parabolic-band approximation, Weiler gives $f(x)$ as

$$
f^{p}(x)=\frac{(2 x-1)^{3 / 2}}{\sqrt{6} x^{5}}\left(4+\frac{29 \cdot \sqrt{2}}{12}\right) .
$$

In the three-band models of Pidgeon and of Weiler, the spin-orbit splitting $\Delta$ never enters any of the equations. Thus the "universal" function $f$ depends on only 
one dimensionless parameter, $\hbar \omega / E_{G}$. By contrast, in the extreme that $\Delta \ll E_{G}$ the spin-orbit splitting contributes to the dispersion of the two lowest valence bands, as Eqs. (2.38) show. Consequently, in this situation a four-band model must be used instead of a three-band model. One difference between the latter model and the fomer is that, in the latter case (i.e., this work), the function $f$ depends upon both $\Delta / E_{G}$ and $\hbar \omega / E_{G}$.

\subsubsection{Exciton Contributions.}

Several authors have considered the effects that excitons have upon two-photon absorption..$^{30,37-40}$ Lee and Fan, ${ }^{30}$ in particular, have considered the case that pertains to rinc blende semiconductors. Lee and Fan show that excitons increase the two-photon absorption coefficient, particularly near the two-photon absorption edge $\left(2 \hbar \omega \approx E_{G}\right)$. Their enhancement function for excitons, $g_{e x}$, is ${ }^{17}$

$$
g_{e x}\left(\frac{\hbar \omega}{E_{G}}\right)=(1+X)^{2} \frac{\pi X e^{\pi X}}{\sinh (\pi X)}\left(\frac{\hbar \omega}{E_{b}} J\right)^{2}
$$

where $E_{b}$ is the binding energy of the exciton and where

$$
\begin{aligned}
& J \equiv 2 Y^{2} \int_{0}^{1} d y \frac{y[(1+y) /(1-y)]^{Y}}{\left[1+(y Y / X)^{2}\right]^{2}} \exp \left[-2 X \tan ^{-1}\left(\frac{y Y}{X}\right)\right] \\
& X \equiv \sqrt{E_{b} /\left(2 \hbar \omega-E_{G}\right)}
\end{aligned}
$$

and

$$
Y \equiv \sqrt{E_{b} /\left(E_{G}-\hbar \omega\right)}
$$

Near the band edge and for $E_{b} \ll E_{G}$, Eq. (2.49) reduces to ${ }^{17}$

$$
g_{e x}=2 \pi\left[E_{b} /\left(2 \hbar \omega-E_{G}\right)\right]^{3 / 2}
$$

This expression clearly shows that excitons contribute to two-photon absorption more strongly as $2 \hbar \omega \rightarrow E_{G}$. Although the exciton enhancement factor is not 
bounded near the two-photon absorption edge, the exciton-enhanced two-photon absorption remains finite, as can be seen by applying Eq. (2.53) to Eqs.(2.42) or (2.47).

\subsection{Tunneling Models of Two-Photon Absorption.}

Several workers have proposed models of two-photon absorption ${ }^{14,41,42}$ that are based upon electron-tunneling. In the tunneling picture, the fields perturb the energy levels of the material system so that two states which are ordinarily greatly separated in energy are driven close enough for electrons to tunnel through the reduced energy barrier. The quantum system is regarded as an entity comprised of material plus fields. The field is treated as an intrinsic part of the system, not as a weak perturbation to the states of the material. Therefore, the wave functions of the total system include an additional time dependence that is not found in the material system alone - namely, a quasi-energy. ${ }^{43,44}$ For harmonic fields such as monochromatic light waves, each quasi-energy of the total system is an energy level of the isolated material plus an overtone that is an integral multiple of $\hbar \omega$. Tunneling occurs when the quasi-energies of two states are nearly equal - i.e., when the difference between the energy of the ground state of the isolated material and the energy of an excited state equals $n \hbar \omega$, where $n$ is an integer.

Weiler, ${ }^{17,41}$ Vaidyanathan et al., ${ }^{12,33-35}$ and Bayfield ${ }^{45}$ have reviewed tunneling models of two-photon absorption. The original tunneling model, which was due to Keldysh, ${ }^{14}$ was shown by Bychkov and Dykhne ${ }^{42}$ to be incorrect for evennumbered photon absorption. Following Ref. 42, Weiler has derived an expression for the coefficient of two-photon absorption. ${ }^{17}$ Weiler's model is a three-band model in which the lower band is douibly degenerate at $k=0$ and the bands are parabolic in $\vec{k}$. Weiler's expression for the coefficient of two-photon absorption is the same as in the previous section, but with $f\left(\hbar \omega / E_{G}\right)$ replaced by the following tunneling 
function:

$$
f^{t}(x)=\frac{f_{0}}{x} \Phi^{\prime}(x, \sqrt{2(2 x-1) / x}),
$$

where

$$
f_{0}=16(4+29 \sqrt{2} / 12) / \sqrt{6}
$$

and

$$
\Phi^{\prime}(x, y)=\frac{3}{\pi^{2}} \sqrt{2 x} e^{-y^{2}} \int_{0}^{y} e^{z^{2}} \sin ^{2}\left(\pi x^{1 / 2} z / 2\right) d z .
$$

Near the two-photon absorption edge $(x \approx 1 / 2)$, the function $\Phi^{\prime}$ approximately equals $(2 x-1)^{3 / 2}$, as for an allowed-forbidden two-photon transition:

$$
\Phi^{\prime}(x, \sqrt{2(2 x-1) / x}) \stackrel{x \rightarrow 1 / 2}{\longrightarrow}(2 x-1)^{3 / 2} .
$$

Weiler has stated that, except very near the two-pioton absorption edge, this model overestimates the two-photon absorption coefficient, $\beta .^{17}$

We shall examine this assertion in Section 2.6 when we compare the models that have been discussed so far to available experimental data for $\mathrm{ZnS}$. First, though, we examine the nonlinear susceptibility tensor formalism in order to round out our review of two-photon absorption.

2.4 Nonlinear Optical Susceptibility Formalism.

In this section, we review the response-function formalism, which is the third major model for two-photon absorption. The formalism is essentially an empirical, macroscopic model, but it can also be derived microscopically. ${ }^{46,47}$ This model is useful for elucidating the macroscopic symmetry properties of the coefficient of twophoton absorption, for introducing local-field corrections, and for showing a simple relation between dual-frequency coefficients of two-photon absorption ( $c f$. Section 2.1.2) and the monochromatic coefficients $\beta$.

The macroscopic response of a non-magnetic, electrically neutral insulator to an externally imposed electromagnetic field can be described by an electric polar- 
ization density $\overrightarrow{\mathbf{P}}(\vec{r}, t)$. To a first approximation, $\overrightarrow{\mathbf{P}}$ is the volumetric density of electric dipole moments in the material. There are no general constraints upon the polarization density other than it must satisfy the macroscopic form of Maxwell's equations. In particular, ${ }^{48}$

$$
\begin{gathered}
\vec{\nabla} \cdot(\overrightarrow{\mathbf{E}}+4 \pi \overrightarrow{\mathbf{P}})=0 \\
\vec{\nabla} \times \overrightarrow{\mathbf{B}}=\frac{4 \pi}{c} \overrightarrow{\mathbf{J}}+\frac{1}{c} \frac{\partial \overrightarrow{\mathbf{E}}}{\partial t}
\end{gathered}
$$

where $\overrightarrow{\mathbf{E}}, \overrightarrow{\mathbf{B}}$, and $\overrightarrow{\mathbf{J}}$ are the macroscopic electric field, the macroscopic magnetic induction, and the macroscopic current density, respectively. From the preceding equations, it follows that $\overrightarrow{\mathbf{P}}$ satisfies the following condition:

$$
\overrightarrow{\mathbf{J}}=\frac{\partial \overrightarrow{\mathbf{P}}}{\partial t}
$$

Since the rate of energy t,ransferred from an electromagnetic field to a unit volume of material is $\overrightarrow{\mathbf{E}} \cdot \overrightarrow{\mathbf{J}},{ }^{49} \mathrm{Eq} .(2.59)$ shows that optical absorption is intimately connected to the polariz tion density. By a suitable constitutive relation, the latter is in turn a function of the macroscopic electric field and thus of optical intensity.

In this section, we restrict attention to quasi-steady-state fields and write the electric field and polarization density at frequency $\omega$ as follows, defining the Fourier components $\vec{E}(\omega)$ and $\vec{P}(\omega)$ :

$$
\begin{aligned}
& \overrightarrow{\mathbf{E}}(\vec{r}, t)=\vec{E}(\omega) e^{-i \omega t}+\text { c.c. } \\
& \overrightarrow{\mathbf{P}}(\vec{r}, t)=\vec{P}(\omega) e^{-i \omega t}+\text { c.c. }
\end{aligned}
$$

In these expressions, the dependence of $\vec{E}(\omega)$ and $\vec{P}(\omega)$ upon $\vec{r}$ is implied. The frequencies, the total electric field and the polarization density are all taken to be real-valued. Consequently, $\vec{E}$ and $\vec{P}$ satisfy the following relations:

$$
\begin{aligned}
& \vec{E}(\omega)=\vec{E}^{*}(-\omega) \\
& \vec{P}(\omega)=\vec{P}^{*}(-\omega)
\end{aligned}
$$


In general, $\vec{P}$ is a superposition of a linear term plus successively higher order nonlinear terms:

$$
\vec{P}=\vec{P}^{(1)}+\vec{P}^{(2)}+\vec{P}^{(3)}+\text { higher order terms. }
$$

If we assume that $\overrightarrow{\mathbf{P}}$ at an arbitrary point $\vec{r}$ in the medium depends only upon $\overrightarrow{\mathbf{E}}$ at $\vec{r}$ and that $\overrightarrow{\mathbf{E}}$ and $\overrightarrow{\mathbf{P}}$ are stationary functions of time, then the $i$-th Cartesian component of the s-th order contribution to $\vec{P}$ is given by the following expression: $:^{50}$

$$
P_{i}^{(s)}(\omega)=\sum_{\omega_{1}} \sum_{\omega_{2}} \ldots \sum_{\omega_{s}} \chi_{i j \ldots s}^{(s)}\left(-\omega ; \omega_{1}, \omega_{2}, \ldots, \omega_{s}\right) E_{j}\left(\omega_{1}\right) E_{k}\left(\omega_{2}\right) \ldots E_{s}\left(\omega_{s}\right)
$$

The tensor $\chi_{i j \ldots s}^{(s)}$ is called the "s-th order nonlinear electric susceptibility tensor." The repeated indices $j, k, \ldots, s$ imply summation over all spatial components of $\overrightarrow{\mathbf{E}}$. Because the indices $j, k, \ldots, s$ and their corresponding frequencies are dummy indices, $\chi^{(s)}$ may be chosen to be invariant when any pair of spatial subscripts and the corresponding frequencies are interchanged. ${ }^{50}$

In Eq. (2.63) each of the dummy frequency arguments $\omega_{1}, \omega_{2}, \ldots, \omega_{s}$ ranges over all frequencies represented in the spectrum of $\overrightarrow{\mathbf{E}}$ subject to the constraint that $\omega_{1}+\omega_{2}+\ldots+\omega_{s}=\omega$. A given spectral component of $\overrightarrow{\mathbf{E}}$ can be repeated in the summation. The fact that the frequency $\omega$ of the induced polarization must equal an harmonic combination of the incident frequencies $\omega_{1} \ldots \omega_{s}$ has some clear physical interpretations. In the wave-optics picture of light, it means that the ncnlinear polarization is due to the redistribution of charge at the beat frequencies of the incident electromagnetic fields. This is called "wave mixing." In the photon picture of light, it means that energy is conserved. The preceding constraint upon the spectral content of $\vec{P}^{(s)}$ means that, for a monochromatic incident field at frequency $\pm \omega$, the lowest-order polarizations at $\omega$ are $\vec{P}^{(1)}$ and $\vec{P}^{(3)}$. These account for oneand two-photon absorption, respectively. In a monochromatic beam, the secondorder nonlinear polarization accounts for processes only at $\pm 2 \omega$ (second harmonic 
generation) and at the null-frequency (optical rectification) and does not contribute directly to two-photon absorption. $\left(\vec{P}^{(2)}\right.$ may, however, contribute indirectly to $\vec{P}^{(3)}$ and thus to $\beta,{ }^{51}$ as we shall discuss in Appendix D.)

To find the relation between the coefficient of two-photon absorption and the nonlinear polarization $\vec{P}^{(3)}$, we recapitulate the definition of optical intensity. The optical intensity is the magnitude of Poynting's vector averaged over many optical cycles:

$$
I=\left|\left\langle\frac{c}{4 \pi} \overrightarrow{\mathbf{E}}(\vec{r}, t) \times \overrightarrow{\mathbf{H}}(\vec{r}, t)\right\rangle\right|
$$

where $\overrightarrow{\mathbf{H}}=\overrightarrow{\mathbf{B}}$ for nonmagnetic materials. The angular brackets in Eq. (2.64) denote a suitable time average. The construct of an optical intensity is useful only if the electromagnetic field is comprised of discrete, nearly monochromatic beams whose amplitudes are slowly varying functions of time. For a single monochromatic plane wave the intensity may be written as

$$
I=\frac{c n}{2 \pi}|\vec{E}(\omega)|^{2} \cos \theta_{0}
$$

where $n$ is the real part of the refractive index at frequency $\omega$ and $\cos \theta_{0}$ is the angle between the real part of the propagation vector $\vec{k}$ and Poynting's vector.

From Poynting's Theorem, we can now express the coefficient of optical attenuation, $\alpha_{o p t}$, in terms of the macroscopic electric field and the polarization density. Poynting's Theorem states that

$$
-\vec{\nabla} \cdot\left(\frac{c}{4 \pi} \overrightarrow{\mathbf{E}} \times \overrightarrow{\mathbf{H}}\right)=\frac{1}{8 \pi} \frac{\partial}{\partial t}(\overrightarrow{\mathbf{E}} \cdot \overrightarrow{\mathbf{E}}+\overrightarrow{\mathbf{H}} \cdot \overrightarrow{\mathbf{H}})+\overrightarrow{\mathbf{J}} \cdot \overrightarrow{\mathbf{E}}
$$

The left-hand side of Eq. (2.63) is conventionally interpreted as the decrease per unit thickness in the energy flux (power per area) across an infinitesimally thin slab. The first term on the right-hand side is interpreted as the rate of change of electric and magnetic energy stored per unit volume of the slab. The last term 
is Joule heating. When Eq. (2.66) is averaged over long but finite times, the first term on the right vanishes because of the slowly-varying amplitude approximation. Rewriting the time-averaged result, one has

$$
-\frac{\partial I}{\partial \zeta}=\langle\overrightarrow{\mathbf{E}} \cdot \overrightarrow{\mathbf{J}}\rangle
$$

where $\zeta$ is a spatial coordinate in the direction of energy propagation. From the definition of the optical attenuation coefficient, which is

$$
\alpha_{o p t} \equiv-\frac{1}{I} \frac{\partial I}{\partial \zeta}
$$

one has

$$
\alpha_{o p t}=\frac{2 \pi}{c n \cos \theta_{0}}\left\langle\overrightarrow{\mathbf{E}} \cdot \frac{\partial \overrightarrow{\mathbf{P}}}{\partial t}\right\rangle /|\vec{E}(\omega)|^{2} .
$$

We note from Eq. (2.69) that for a monochromatic field $\alpha_{o p t}$ is zero if $\overrightarrow{\mathbf{P}}$ is in phase with $\overrightarrow{\mathbf{E}}$. That is, optical absorption in non-magnetic dielectrics is a consequence of the existence of a time-varying polarization density which has a component in phase-quadrature with respect to the macroscopic electric field. ${ }^{15,48}$

From Eq. (2.63) the first- and third-order polarizations for a monochromatic wave are

$$
\begin{aligned}
& P_{i}^{(1)}=\chi_{i j}^{(1)}(-\omega ; \omega) E_{j}(\omega) \\
& P_{i}^{(3)}=3 \chi_{i j k l}^{(3)}(-\omega ; \omega, \omega,-\omega) E_{j}(\omega) E_{k}(\omega) E_{l}^{*}(\omega) .
\end{aligned}
$$

The factor of 3 in Eq. (2.70) comes from summing over all electrical field components in the definition of the s-th order polarization (Eq. (2.63)) and from the permutation symmetry of $\chi^{(3)} .^{50}$ The time-average of $\overrightarrow{\mathbf{E}} \partial \overrightarrow{\mathbf{P}} / \partial t$ for steady-state monochromatic fields is the real part of $-2 i \omega \vec{E}^{*} \cdot \vec{P}^{49}$ Inserting this relation and Eq. (2.70) into Eq. (2.69) yields

$$
\alpha_{o p t}=\alpha+\beta I
$$

where

$$
\alpha=\frac{4 \pi \omega}{c n \cos \theta_{0}|\vec{E}|^{2}} \times \operatorname{Im}\left[E_{i}^{*}(\omega) \chi_{i j}^{(1)}(\omega) E_{j}(\omega)\right]
$$


and

$$
\beta=\frac{24 \pi^{2} \omega}{c^{2} n^{2} \cos ^{2} \theta_{0}|\vec{E}|^{4}} \times \operatorname{Im}\left(E_{i}^{*}(\omega) \chi_{i j k l}^{(3)}(-\omega ; \omega, \omega,-\omega) E_{j}(\omega) E_{k}(\omega) E_{l}^{*}(\omega)\right)
$$

In Eq. (2.72a), $\chi^{(1)}(-\omega ; \omega)$ has been written as $\chi^{(1)}(\omega)$, in keeping with common convention, and (as before) the repeated spatial subscripts imply a summation.

Although the magnitude of the electric field does not contribute to the absorption coefficients in Eq. (2.72), the orientation of the field does. That is, the effective absorption coefficients $\alpha$ and $\beta$ depend upon the optical polarization and the direction of propagation. It is convenient to define a set of direction cosines $c_{j}$ and a set of phases $\phi_{j}$ such that each field component $E_{j}$ is the product of the field strength $|\vec{E}|$ times a direction cosine times an exponential phase factor:

$$
E_{j}=|\vec{E}| c_{j} e^{-i \phi_{j}}
$$

We can then define the "effective susceptibility tensors" $\chi_{e f f}^{(1)}$ and $\chi_{e f f}^{(3)}$ as follows:

$$
\begin{aligned}
& \chi_{e f f}^{(1)}=\sum_{i, j} \chi_{i j}^{(1)} c_{i} c_{j} e^{i\left(\phi_{i}-\phi_{j}\right)} / \cos \theta_{0} \\
& \chi_{e f f}^{(3)}=\sum i, j, k, l \chi_{i j k l}^{(3)} c_{i} c_{j} c_{k} c_{l} e^{i\left(\phi_{i}-\phi_{j}-\phi_{k}+\phi_{l}\right)} / \cos ^{2} \theta_{0} .
\end{aligned}
$$

For monochromatic beams, the one- and two-photon absorption coefficients may then be written as

$$
\alpha=\frac{4 \pi \omega}{c n} \operatorname{Im}\left(\chi_{e f f}^{(1)}\right)
$$

and

$$
\beta=\frac{24 \pi^{2} \omega}{c^{2} n^{2}} \operatorname{Im}\left(\chi_{e f f}^{(3)}\right)
$$

Equation (2.75b) shows that the two-photon absorption coefficient depends upon $\chi^{(3)}$, as Bloembergen has previously stated. (See Eq. (5-25) of Ref. 15.) 


\subsubsection{Polarization-Dependence of $\beta$.}

In many applications it is desirable to maximize or minimize $\chi_{e f f}^{(3)}$. Two-photonpumped optical devices and phase-conjugate optical devices require a maximum $\chi_{\text {eff }}^{(3)}$, for instance, and optical elements in high-power lasers require a minimum $\chi_{\text {eff }}^{(3)}$. As Eqs. (2.76)-(2.78) will show, $\chi_{\text {eff }}^{(3)}$, and hence $\beta$, lepends upon the polarization of the incident beam even in cubic or isotropic materials. ${ }^{52,53}$ Consequently, there is a set of polarizations for which two-photon absorption is a maximum and a set for which it is a minimum.

The spatial symmetry properties of $\chi^{(1)}$ and $\chi^{(3)}$ establish the polarizationdependence of $\alpha$ and $\beta$, respectively. One may use either of two approaches to find the symmetry properties of interest. One may apply group theory to the quantum mechanical transition rates given in Eqs.(2.1) and (2.11),, $454-56$ or else one may apply Neumann's Postulate to the macroscopic susceptibility tensors..$^{50,52,57}$ Neumann's Postulate states that every intrinsic property of a crystal must remain invariant under the action of each element of the point group to which that crystal belongs. ${ }^{57}$ While group theory is more elegant than an application of Neumann's Postulate, the latter method is quite straight-forward. Further, it is also simple for solids of high spatial symmetry.

Spatial symmetry properties reduce the number of independent parameters in the susceptibility tensors. Although $\chi_{i j k l}^{(3)}$ has 81 spatial components, ${ }^{50}$ most of them vanish for materials of high spatial symmetry, and the terms that do not vanish are strongly inter-related. For monochromatic waves in isotropic materials, for example, $\chi^{(3)}$ has only two independent components, ${ }^{50}$ which can be denoted as $\chi_{1122}^{(3)}$ and $\chi_{1221}^{(3)}$. Under the same circumstances in each of the cubic groups $m 3 m$, $\overline{4} 3 m$, and $432, \chi^{(3)}$ has only three independent components. ${ }^{50}$ These can be denoted $\chi_{1122}^{(3)}, \chi_{1221}^{(3)}$ and $\chi_{1111}^{(3)}$. (The point groups just mentioned are distinguished from 
the remaining cubic point groups by the fact that the latter do not possess a fourfold axis of rotational symmetry. These two remaining cubic point groups have the additional components $\chi_{2121}^{(3)}$ and $\chi_{2112}^{(3)}$.) The subscripts 1 and 2 refer to any two principal axes in the material.

We now examine the form of $\chi_{e f f}^{(3)}$ in materials that are either isotropic or are cubic with four-fold axes of symmetry. The effective susceptibility tensors defined by Eq. (2.74) may be written in the following form:

$$
\begin{aligned}
\chi_{e f f}^{(3)}= & {\left[\chi_{1221}^{(3)}+2 \chi_{1122}^{(3)}\right] } \\
& -\left[4 \chi_{1221}^{(3)}\right]\left(c_{1}^{2} c_{2}^{2} \sin ^{2}\left(\phi_{1}-\phi_{2}\right)+c_{2}^{2} c_{3}^{2} \sin ^{2}\left(\phi_{2}-\phi_{3}\right)+c_{3}^{2} c_{1}^{2} \sin ^{2}\left(\phi_{3}-\phi_{1}\right)\right] \\
& +\left[\chi_{1111}^{(3)}-\chi_{1221}^{(3)}-2 \chi_{1122}^{(3)}\right]\left(c_{1}^{4}+c_{2}^{4}+c_{3}^{4}\right) .
\end{aligned}
$$

(In isotropic materials, the third term in square brackets in Eq. (2.76) vanishes by symmetry ${ }^{50,52}$ ) This simplifies the expression for $\beta$. Also, simplification is possible if the incident field is linearly polarized, because the sine terms all vanish, leaving

Linear Polarization:

$$
\begin{aligned}
\chi_{e f f}^{(3)} & =\left[\chi_{1221}^{(3)}+2 \chi_{1122}^{(3)}\right] \\
& +\left[\chi_{1111}^{(3)}-\chi_{1221}^{(3)}-2 \chi_{1122}^{(3)}\right]\left(c_{1}^{4}+c_{2}^{4}+c_{3}^{4}\right)
\end{aligned}
$$

Similarly, if the incident field is circularly polarized and is perpendicular to a principal axis, one has

Circular Polarization:

$$
\chi_{e f f}^{(3)}=\left[\chi_{1111}^{(3)}-\chi_{1221}^{(3)}+2 \chi_{1122}^{(3)}\right] / 2
$$

Next, we consider the case of linearly polarized light propagating through a polycrystalline medium in which the crystallites are oriented completely randomly. The result will be used in the next chapter. We make three simplifying assumptions. First, the angular distribution of orientations is uniform over a sphere. Second, the 
crystallites are macroscopic but small enough so that the beam travels through a very large number of them, sampling all possible orientations of the crystal axes many times. Finally, we neglect any induced bircfringence. Averaging Eq. (2.76) over $4 \pi$ steradians gives the following effective nonlinear susceptibility for this case:

Random Crystallites:

$$
\chi_{e f f}^{(3)}=\frac{3}{5} \chi_{111}^{(3)}+\frac{2}{5} \chi_{1221}^{(3)}+\frac{4}{5} \chi_{1122}^{(3)}
$$

In reality, neglecting the induced birefringence may be a poor assumption. In a material with a large value of $\chi^{(3)}$, appreciable birefringence may occur in the presence of beams that are intense enough to induce two-photon absorption. ${ }^{58}$ If the beam travels through many randomly oriented crystallites, undergoing many random phase shifts, then the effective nonlinear susceptibility is determined from Eq. (2.76) with each $\operatorname{sine}^{2}$ term replaced by its average value, $1 / 2$. This yields Random Polarizalion:

$$
\chi_{e f f}^{(3)}=\frac{3}{5} \chi_{1111}^{(3)}+\frac{4}{5} \chi_{1122}^{(3)}
$$

The polarization dependence of $\beta$ follows in an obvious way from the symmetry of $\chi_{e f f}^{(3)}$ in the four preceding equations and from the expession for $\beta$, Eq. $(2.75 \mathrm{~b})$.

\subsubsection{Local-Field Corrections.}

The local field acting on an electron in an isolated solid is the superposition of the fields due to all other charges in the solid plus all external fields that may be present. That is, it is the total field. Since the instantaneous fields that act upon an electron fluctuate rapidly in time as the electron dances in its microscopic orbit, the local field is generally taken as a time-average of the instantaneous total field at the location of the electron. The averaging time is chosen as an arbitrary, convenient duration that is short compared to undulations in the external field but long compared to the average period of the electronic motion. The strongest 
contributions to the local field come from the charges nearest the electron under consideration. In transparent media, neighboring charges are bound, and so the local field is essentially a correction for dipole-dipole interactions.

Bloembergen ${ }^{15}$ and Armstrong et al. ${ }^{46}$ have applied the classical derivation due to Lorentz and Lorenz to nonlinear polarization densities. The basic result is that the macroscopic electric field amplitude $\vec{E}(\omega)$ can be replaced by the local field times a Lorentz correction factor $L(\omega)$. That is,

$$
\vec{E}(\omega)=L(\omega) \vec{E}_{l o c}(\omega)
$$

where, for a cubic or isotropic array of polarizable entities (bonds, atoms, or molecules)

$$
L(\omega)=\frac{1+4 \pi \chi^{(1)}(\omega)}{3}
$$

Equation (2.82) neglects corrections due to higher order nonlinearities, which are typically several orders of magnitude smaller than $\chi^{(1)}$. The macroscopic susceptibility $\chi^{(s)}$ is then a microscopic polarizability times a product $L^{*}(\omega) L\left(\omega_{1}\right) \ldots L\left(\omega_{s}\right)$. In particular,

$$
\chi^{(3)}(-\omega ; \omega, \omega,-\omega)=|L(\omega)|^{4} \aleph^{(3)}(-\omega ; \omega, \omega,-\omega)
$$

where $N^{(3)}$ is a third-order microscopic polarizability.

In nonlinear refraction, local-field effects have been shown to be important far below any optical resonances and far above phonon resonances. ${ }^{59}$ (Nonlinear refraction is the counterpart to nonlinear absorption. The nonlinear refractive index of a material is proportional to the real part of $\chi_{e f f}^{(3)}$ just as the two-photon absorption coefficient is proportional to the imaginary part.) The nonlinear refractive index of many transparent solids (oxides, especially) appears to be primarily a function of the linear refractive index and the linear dispersion according to Boling, Glass and Owyoung. ${ }^{59}$ This function is derived from the Lorentz-Lorenz law, 
which relates the dispersion of the linear index of refraction to local-field corrections.

In crystals the situation is different for two-photon absorption than it is for nonlinear refraction. First, if two-photon absorption occurs, then the optical frequency is not far below all optical resonances. Second and more importantly, a two-photon transition elevates an electron from a valence state to a de-localized conduction state. In the conduction band, the electron interacts with a large number of dipoles, thus averaging out the spatial fluctuations of the local field. The Lorentz correction $L$ should approach unity for highly de-localized electrons in ideal crystals, as Bloembergen has argued. ${ }^{15}$

In highly transparent glass, unlike crystals, local-field effects should be important for the first excited electronic state. This is because the aperiodic structure of glasses does not permit an excited electron to migrate easily. Above the band edge,

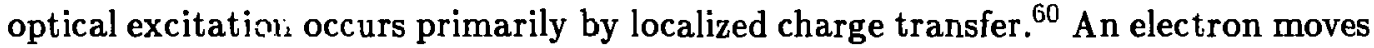
from its lowest energy state on an anion like o.ygen to a cation like silicon, boron, or phosphor or even to an interstitial, impurity, or defect. According to Eq. (2.83), the local-field corection lowers the macroscopic nonlinear polarizability in glasses, since $\left(1+4 \pi \chi^{(1)}\right) / 3 \sim 0.70$ in them. Therefore, based on local-field considerations only, one might expect glasses to exhibit a $\beta$ that is $2-4$ times less than $\beta$ in crystals that are chemically similar.

\subsection{Frequency Dependence of the Two-Photon Absorption Coefficient.}

In this section, we consider two aspects of the frequency dependence of twophoton absorption. First, we discuss the dispersion of $\beta$. Then, we discuss the relation between monochromatic and dual-frequency coefficients of two-photon absorption. 


\subsubsection{Dispersion of $\beta$.}

Sections 2.1-2.2 gave several equations for two-photon absorption, which upon closer scrutiny proved to be quite similar. This was not surprising, since all derivations started from Eq. (2.10) and since they all were supposed to model zinc blende materials. With such similarity among results, one might think that the dispersion of $\beta$ is understood. This is not quite so. We have not yet considered whether the momentum matrix elements may depend indirectly upon frequency. They do. this is evident because a matrix element $P_{m n}=[\vec{p}]_{m n}$ connects two Bloch orbitals $\phi_{m}^{*}\left(\vec{k}_{i}\right)=\left\langle E_{m}\left(\vec{k}_{i}\right), \vec{k}_{i}\right|$ and $\phi_{n}\left(\vec{k}_{i}\right)=\left|E_{n}\left(\vec{k}_{j}\right), \vec{k}_{j}\right\rangle$. The constraints of conservation of energy and momentum determine what $E_{m}$ and $\vec{k}_{i}$ can be, assuming the optical wave and the initial state $\phi_{n}\left(\vec{k}_{j}\right)$ are given. Thus, $P_{m n}$ depends implicitly on the optical frequency and the optical wavevector. Of these two factors, the wavevector contribution to $P_{m n}$ is neglible in the electric-dipole approximation, as we now show.

By conservation of wave momentum, the momentum matrix elements of a direct transition, vanish unless

$$
\vec{k}_{i}-\vec{k}_{j}=\vec{k}_{o p t}+\vec{G}
$$

Here $\vec{k}_{i}$ and $\vec{k}_{j}$ are electron wave vectors lying in the first Brillouin Zone, $\vec{k}_{\text {opt }}$ is the optical wave vector, and $\vec{G}$ is a reciprocal lattice vector. (Adler ${ }^{61}$ has shown that $\vec{G}$ is zero when the polarization density at a point $\vec{r}$ depends only on the fields at $\vec{r}$.) Because the optical wave vector satisfies the relation

$$
\left|\vec{k}_{\text {opt }}\right|=\omega n / c
$$

the difference $\vec{k}_{i}-\vec{k}_{j}$ for two siates involved in an optical transition depends indirectly upon the optical frequency $\omega$. Therefore the momentum matrix element between states $\phi_{m}$ and $\phi_{n}$ also depends upon $\omega$. According to Eq. (2.84), expanding 
the wave function of the state $\phi_{n}$ in a series of powers of the optical wave vector yields

$$
\phi_{n}\left(\vec{k}_{i}\right)=\phi_{n}\left(\vec{k}_{j}\right)+\vec{k}_{o p t} \cdot \vec{\nabla}_{\vec{k}} \phi_{n}+\text { higher-order terms. }
$$

Matrix elements that are explict functions of $\vec{k}_{\text {opt }}$ have the same form as higherorder multipole moments. Within the model of the electric dipole formalism, such terms may be disregarded, because they depend upon various derivatives of the field amplitude.

Before mentioning a way to cope with the dispersion of the transition matrix elements, we recall an alternative description of transition moments that is used successfully in atomic spectroscopy, namely the optical transition strength $f_{i j}$. This quantity is defined in terms of the momentum matrix elements $P_{i j}$ as follows: ${ }^{62}$

$$
f_{i j}=2\left|P_{i j}\right|^{2} / m E_{i j}
$$

Other workers such as Braunstein and Ockman ${ }^{24}$ and Vaidyanathan et al. ${ }^{34,35}$ have given expressions for $\beta$ using optical transition strengths rather than momentum matrix elements. Let us present an expression for $\beta$ in terms of $f_{i j}$, using the simple model that was discussed in Section 2.1.3. In that model, the total twophoton absorption coefficient was taken as a sum of two-band coefficients $\beta_{c v}$. In each two-band coefficient, the intermediate state of the electron was taken to be either in the initial band of the particular transition, $v$, or in the final band, $c$. Recalling the result from Section 2.1.3 that

$$
\beta=\frac{2^{11 / 2} \pi e^{4}}{3 m^{2}(\hbar \omega)^{5}} \frac{\left[2 \hbar \omega-E_{G}\right]^{3 / 2}}{n^{2} c^{2}} \times \sum_{v=v_{1}, v_{2}} m_{c v}^{1 / 2}\left|P_{c v}\right|^{2},
$$

we see that, using Eq. (2.87) and the fact that $E_{c \vartheta}=2 \hbar \omega-E_{G}$, the expression for $\beta$ becomes

$$
\beta=\frac{2^{11 / 2} \pi e^{4}}{3 m(\hbar \omega)^{5}} \frac{\left[2 \hbar \omega-E_{G}\right]^{5 / 2}}{n^{2} c^{2}} \times \sum_{v=v_{1}, v_{2}} m_{c v}^{1 / 2} f_{c v} / 2 .
$$


Note that Eqs. (2.28) and (2.88) are similar but not identical. Both equations predict that $\beta$ will asymptotically approach zero at high frequency, and importantly both predict that $\beta$ has an absolute maximum for $E_{G} / \hbar>\omega>E_{G} / 2 \hbar$. However, they depend upon $\omega$ differently. The former varies explicitly as the $3 / 2$ power of $2 \hbar \omega-E_{G}$, but the latter varies as the $5 / 2$ power. The former result is based upon the tacit assumption that $\boldsymbol{P}_{c v}$ does not vary rapidly with Bloch vector, and the latter result presumes the same for $f_{c v}$. Which result is more correct? The predicted frequency dispersion of $\beta$ depends upon the answer.

This brings us back to the necessity of accounting for the dispersion of the effective transition moment if we are really to know how $\beta$ varies with frequency in a given material. One way to estimate this is to calculate the exact wave functions and the corresponding matrix elements numerically. In the next chapter, we shall do this with an empirical pseudopotential approach for $\mathrm{ZnS}$.

\subsubsection{Relation Between $\beta(\omega)$ and $\beta\left(\omega_{1}, \omega_{2}\right)$.}

Because both single-frequency and dual-frequency measurements of two-photon absorption are common, it is necessary to have an approximate method for relating one type of measurement to the other.

Consider a single-beam measurement at frequency $\omega$ and a dual-beam measurement at $\left(\omega_{1}, \omega_{2}\right)$. Suppose that in both cases the sum of the energies of two photons has the same value. That is,

$$
\approx \omega=\omega_{1}+\omega_{2}
$$

Following the discussion that lea to Eq. (2.69), let $\beta\left(\omega_{1}, \omega_{2}\right) I_{1} I_{2}$ denote the total power absorbed per unit volume from beams whose frequencies are $\omega_{1}$ and $\omega_{2}$. Equating this to the rate that heat is dissipated, one obtains

$$
I_{1} I_{2} \beta\left(\omega_{1}, \omega_{2}\right)=2 \operatorname{Re}\left(-i \omega_{1} \vec{P}^{(3)}\left(\omega_{1}\right) \cdot \vec{E}^{*}\left(\omega_{1}\right)-i \omega_{2} \vec{P}^{(3)}\left(\omega_{2}\right) \cdot \vec{E}^{*}\left(\omega_{2}\right)\right)
$$


The nonlinear polarizations $\vec{P}^{(3)}\left(\omega_{1}\right)$ and $\vec{P}^{(3)}\left(\omega_{2}\right)$ are given by

$$
\begin{gathered}
P_{i}^{(3)}\left(\omega_{1}\right)=6 \chi_{i j k l}^{(3)}\left(-\omega_{1} ; \omega_{1}, \omega_{2},-\omega_{2}\right) E_{j}\left(\omega_{1}\right) E_{k}\left(\omega_{2}\right) E_{l}^{*}\left(\omega_{2}\right) \\
\text { and } \\
P_{i}^{(3)}\left(\omega_{2}\right)=6 \chi_{l k j i}^{(3)}\left(-\omega_{2} ; \omega_{2}, \omega_{1},-\omega_{1}\right) E_{k}\left(\omega_{2}\right) E_{j}\left(\omega_{1}\right) E_{i}^{*}\left(\omega_{1}\right) .
\end{gathered}
$$

The factor of 6 arises because, in $\chi^{(3)}$, all the frequency arguments to the right of the semicolon are different. ${ }^{58}$ As in Section 2.4, it is convenient to eliminate the magnitudes of the fields from Eq. (2.90) and (2.91) by defining an effeciive nonlinear susceptibility, which we denote as $\chi_{e f f}^{(3)}\left(\omega_{1}, \omega_{2}\right)$. Then $\beta\left(\omega_{1}, \omega_{2}\right)$ is given by the following expression:

$$
\beta\left(\omega_{1}, \omega_{2}\right)=\frac{48 \pi^{2}\left(\omega_{1}+\omega_{2}\right)}{c^{2} n_{1} n_{2}} \operatorname{Im}\left(\chi_{\epsilon f f}^{(3)}\left(\omega_{1}, \omega_{2}\right)\right) .
$$

We now make an approximation that is not rigorously correct but is useful for obtaining a first estimate of the relation between single-beam and dual-beam twophoton absorption. We invoke Kleinman symmetry. ${ }^{50}$ Kleinman symmetry applies to the situation where all frequencies are less than aliy resonant frequencies of a material. In this case the frequency arguments of $\chi^{(3)}$ are all virtually zero. Consequently, Kleinman symmetry implies that

$$
\chi_{i j k l}^{(3)}(-\omega ; \omega, \omega,-\omega)=\frac{1}{4} \chi_{i j k l}^{(3)}\left(-\omega_{1} ; \omega_{1}, \omega_{2},-\omega_{2}\right)
$$

The factor of $1 / 4$ is required in order that $I_{1} I_{2} \beta\left(\omega_{1}, \omega_{2}\right) \rightarrow I^{2} \beta(\omega)$ when $\omega_{1}, \omega_{2} \rightarrow \omega$, because $\chi^{(3)}$ is a discontinuous function of frequency. As a result of Eq. (2.93), the ratio of two-photon absorption coefficients for the single-beam case versus the dualbeam case is approximately as follows:

$$
\frac{\beta(\omega)}{\beta\left(\omega_{1}, \omega_{2}\right)}=\frac{2 \omega n_{1} n_{2}}{\left(\omega_{1}+\omega_{2}\right) n^{2}} \xi, \quad\left(\omega_{1}+\omega_{2}=2 \omega\right)
$$


where the factor $\xi$ accounts for differences in orientations of the various E-fields. It is approximately unity. It is exactly unity whenever all three fields $\left(\vec{E}(\omega), \vec{E}\left(\omega_{1}\right)\right.$, and $\left.\vec{E}\left(\omega_{2}\right)\right)$ are polarized similarly. That is, if all beams are linearly polarized in the same direction or if they all have the same helicity about a common axis, then $\xi=1$. As a special case, for linearly polarized beams in isotropic or cubic materials with four-fold axes of rotation,

$$
\xi=\frac{1-\xi_{0}\left(\left(c_{1}^{\omega}\right)^{4}+\left(c_{2}^{\omega}\right)^{4}+\left(c_{3}^{\omega}\right)^{4}\right)}{1-\xi_{0}\left(\left(c_{1}^{\omega_{1}}\right)^{2}\left(c_{1}^{\omega_{2}}\right)^{2}+\left(c_{2}^{\omega_{1}}\right)^{2}\left(c_{2}^{\omega_{2}}\right)^{2}+\left(c_{3}^{\omega_{1}}\right)^{2}\left(c_{3}^{\omega_{2}}\right)^{2}\right)},
$$

with

$$
\xi_{0} \equiv \frac{\chi_{1111}^{(3)}}{3 \chi_{1122}^{(3)}}-1
$$

In isotropic matter, $\xi_{0}$ identically vanishes. The direction cosines $c_{i}^{\omega_{1}}$ and $c_{i}^{\omega_{2}}$ give the direction of polarization for the fields at $\omega_{1}$ and $\omega_{2}$ in the $i$-th direction. $(i=\mathbf{x}$, $\mathbf{y}$, or $\mathbf{z}$ )

Equation (2.94) is the approximate relationship between single-beam and dualbeam two-photon absorption coefficients.

2.6 Comparison Between Previous Theories and Experiments for ZnS.

Panizza $^{63}$ first reported the 2PA spectrum of $\mathrm{ZnS}$, measuring absolute absorption at room temperature. Panizza used a dual-beam technique with an intense $1.06 \mu \mathrm{m}$ laser pulse and a weak beam from a monochromator. The wavelength and amplitude of the laser pulse were fixed, and the wavelength of the weak beam was varied. The effective absorption coefficient was determined as a function of two-photon energy over the range $3.6-4.0 \mathrm{eV}$. Panizza did not report the polarization of the beams or the orientation of the crystal. Park and Waff, ${ }^{64}$ using the same technique with an intense $0.69 \mu \mathrm{m}$ laser, subsequently reported absolute absorption spectra at $10 \mathrm{~K}, 77 \mathrm{~K}$, and room temperature. Park and V''a ${ }^{\text {q }}$ did report the polarization of the two beams and the orientation of the sample. Their measurements covered the two-photon spectral range 3.55-4.6 eV. Later Bae, Song, 
and $\mathrm{Kim}^{19}$ measured relative two-photon absorption photoacoustically from 3.6$5.4 \mathrm{eV}$ by using a single tuneable dye laser. Recently, van Stryland et al. ${ }^{65}$ have reported measurements at $0.532 \mu \mathrm{m}$ for $2 \mathrm{PA}$ in polycrystalline zinc blende that was manufactured by chemical vapor deposition. Other workers ${ }^{21,66}$ have measured the two-photon absorption coefficient of $\mathrm{ZnS}$ at various isolated spectral points, obtaining a wide range of values for the coefficierts. This justifies taking another look at the previous data.

Zinc sulfide occurs in both cubic (zinc blende) and hexagonal (wurtzite) structures. The basic structural unit in both cases is a tetrahedron of sulfur ions with a zinc ion at the center. Because the nearest-neighbor interaction is strong, the band-gap energies and the optical properties of both the cubic and the hexagonal phases are similar, ${ }^{20}$ as was discussed earlier in this chapter. Single macroscopic crystals of pure wurtzite are readily available, and so most two-photon absorption measurements in $\mathrm{ZnS}$ have been for wurtzite. The cubic phase of $\mathrm{ZnS}$ tends to form only in small platelets or in polycrystalline form. No two-photon absorption spectra exist for single-crystal cubic $\mathrm{ZnS}$.

Figure 2-6 shows the best available measured two-photon absorption spectra for $\mathrm{ZnS}$. The temperature dependent shift of the two-photon absorption edge is plainly evident by comparing the data of Bae, Song, and Kim or Panizza to that of Park and Waff. Isolated, single-point measurements have deliberately been excluded from the figure. Also not shown are the room-temperature curves that Park and Waff obtained. These data agree closely with Panizza's spectrum. Panizza's spectrum is, therefore, used as the standard room-temperature curve. From Eq. (2.94), the relative data of Bae et al. can be absolutely calibrated to Panizza's result. This has already been done in Fig. 2-6. By comparison to all other data, that of Galanin and Chizhikova has a relatively flat spectral dependence, and it is low. We, therefore, 


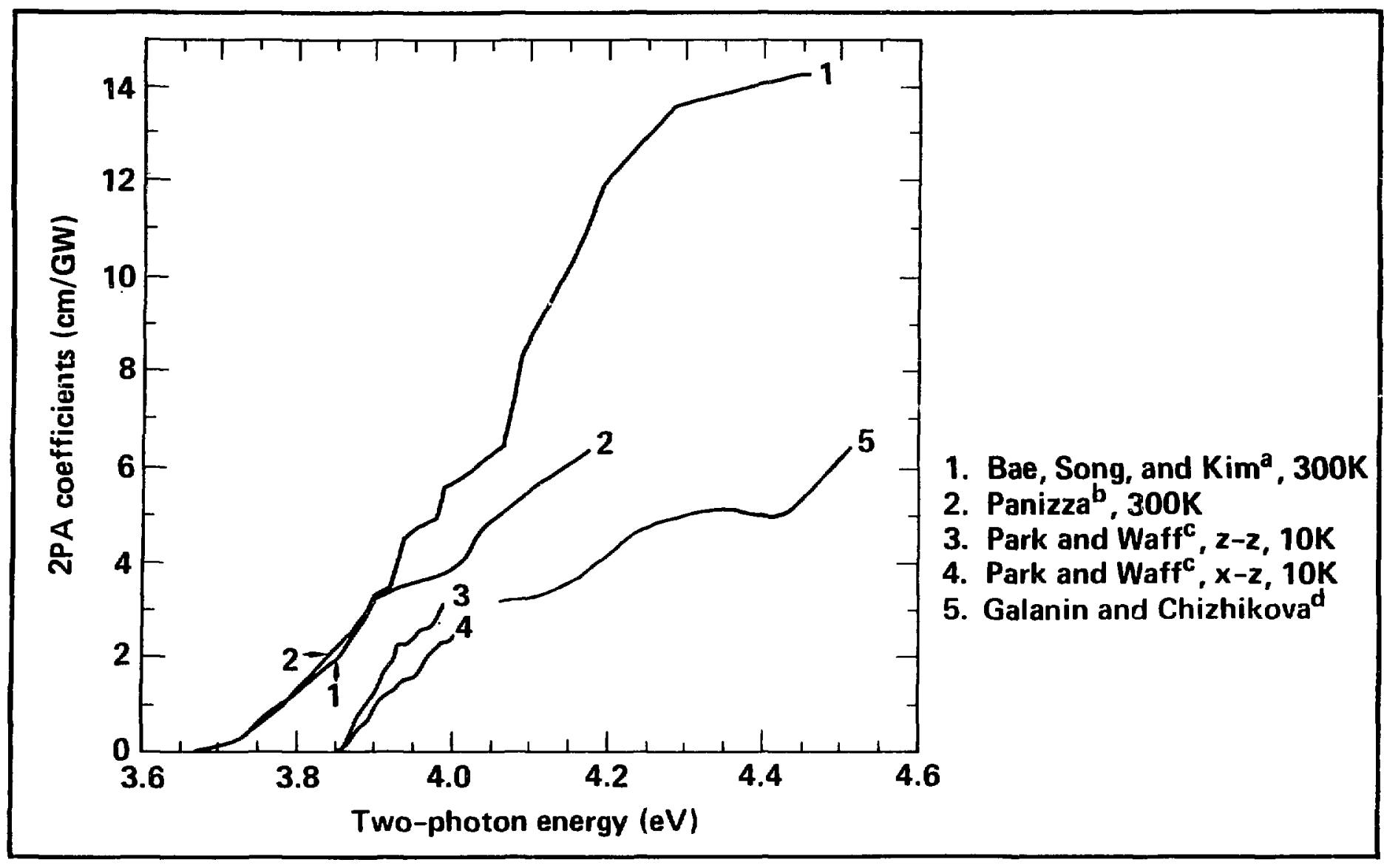

Figure 2-6. Measured Two-Photon Absorption Spectra in ZnS.

"Ref. 19 "Ref. 63 "Ref. 6il "Ref. 21 
believe that it is not reliable. Having calibrated the relative data of Bae et al., we now have experimental values of two-photon absorption coefficients for two-photon energies between $3.5 \mathrm{eV}$ and $5.4 \mathrm{eV}$.

In order to cornpare the theories that we have discussed to the available measured data, we must specify three material parameters: $E_{G}, n$, and $P$. At room temperature, $E_{G} \sim 3.66 \mathrm{eV}$ in $\mathrm{ZnS},{ }^{65} n \sim 2.36 \pm 0.05,{ }^{67}$ and $P$ can be determined from reduced-mass data, according to Eq. (2.45). From the work of Wheeler and Miklosz, ${ }^{68}$ we obtain $m_{c v}=0.18 \mathrm{~m}$, which gives $2 P^{2} / m=20.3 \mathrm{eV}$. This latter value agrees well with the value of $20.4 \mathrm{eV}$ cited recently by Van Stryland et al. ${ }^{65}$

Figure 2-7 compares experimental results to the theories that have been discussed so far. The experimental results are three to six times greater than the theoretical predictions. A the moment we have no way of telling why the disparity exists. Experimental values suffer, because processes other than two-photon absorption can occur, giving high results. Self-focusing and excited-state absorption are two examples. Theoretical values are limited by the inability of the models that have been discussed so far to account for polarization dependence or detailed band shape. Also, excitonic corrections have not been included in the room-temperature predictions. Above all, the measured values shown in Figs. 2-6 and 2-7 are for wurtzite, but the theory is for zinc blende. Therefore, one might think that the two materials should not compare owing to their structural differences. Let us defer judgment on this until we see the pseudopotential predictions in the next chapter.

\subsection{Two-Photon Absorption in Optical Glasses.}

Structurally and chemically, optical glasses are much more complex than the zinc blende compounds that we have considered so far. It is beyond the scope of this work to analyze such materials in depth, and so this section is mostly qual- 


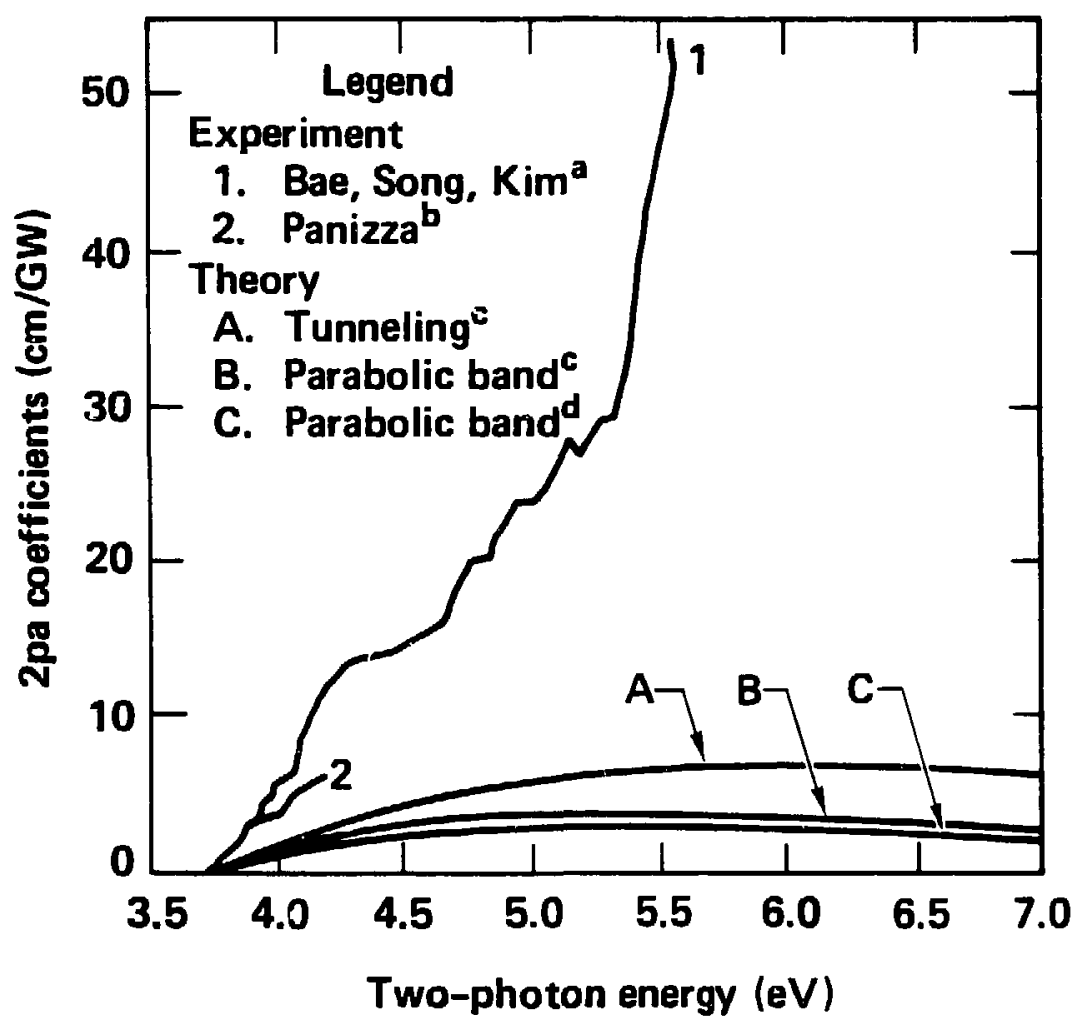

Figure 2-7. Comparison of Theoretical and Measured Values of TwoPhoton Absorption in $\mathrm{ZnS}$.

a Ref. 19.

"Ref. 63.

'Ref. 17.

"This work. 
itative. After we review some of the main characteristics of transparent glasses, we shall show that it is not unreasonable to expect two-step absorption to have an effective two-photon absorption coefficient on the order of a few $\mathrm{cm} / \mathrm{TW}$ in highly transparent glasses. We shall also show that the maximum value that we expect for $\beta$ in highly transparent glasses should be only about an order of magnitude less than that for $\mathrm{ZnS}$, based on energy-gap considerations. However, due to the lack of a well defined energy gap in glasses, we cannot make rigorous estimates of the size of $\beta$.

Structurally, the biggest difference between glasses and simple crystals is the absence of long-range order in glass. This is caused by variations in bond angles, bond lengths, and coordination number. An important consequence of these variations is that the local electric field varies randomly from site to site in the glass, smearing the energy levels and electronic states of each chemical constituent. Spectroscopically, these smeared energy levels give rise to a long absorption tail that declines exponentially as a function of frequency. ${ }^{69}$ In a glass, according to this argument, there should no forbidden energy gap as in a crystal. The density of electronic states in what would correspond to the energy gap should be small but not zero. We shall return to this point momentarily. Another ramification of the structural disorder is the occurrence of dangling bonds and trapped charges. These structural defects, or "color centers," behave like impurity ions with a set of energy levels that is completely diferent than the set of levels associated with properly conjugated bonds.

Chemically, optical glasses are usually complex. While some glasses have only two chemical constituents - as, for example, $\mathrm{SiO}_{2}$ and $\mathrm{As}_{2} \mathrm{~S}_{3}$ - most optical glasses contain many compounds. It is not unusual for the number of elements in a fine optical glass to be between six and ten. Adding small quantities of various ions 
to a glass dramatically alters the number of dangling bonds and trapped charges, allowing the glass scientist to tailor the material properties. It makes accurate, detailed quantum optical predictions extremely difficult, though.

Returning to the issue of whether amorphous materials can have a band gap, recent experiments have shown that a gap can exist in chemically pure amorphous materials. Tests on thick films of annealed, amorphous Ge show only slight differences between amorphous and crystalline absorption edges. ${ }^{60,69}$ Adler has stated that the absorption edge in $\mathrm{Si}$ or Ge may actually be sharper in the amorphous phase than in the crystals. ${ }^{70}$

What happens to the states that should be in the gap? Tauc, ${ }^{69}$ citing unpublished work of Anderson, conjectures that they are squeezed out. This occurs, according to Tauc, because of a Franck-Condon effect. The presence of electrons or other unattached ions influences the position of the lattice ions. The ionic lattice must deform in order to accomodate the new electronic charge distribution of the excited state. This requires additional energy. Thus, the deep states that should lie at the center of the gap are pushed out of the gap. States that do exist in the gap must be due to impurities rather than to "intrinsic" defects, Tauc argues.

The principal contaminant in optical glass is iron. ${ }^{60} \mathrm{Fe}^{3+}$ in alkali silicate glasses leads to a greenish brown color, as may be seen in pyrex glassware, for instance. This coloration is due to the minimum in the absorption spectrum of $\mathrm{Fe}^{3+}$ that occurs around $0.55 \mu \mathrm{m}$. Even in concentrations of a few parts per million, iron dramatically affects the absorption edge of oxide glasses, moving it to longer wavelengths: For highly transparent glasses, it is conceivable that impurity-related two-step absorption will dominate any two-photon absorption that might occur, because the oscillator strengths of impurities such as iron are large, of the order of $0.1 .^{60}$

Let us estimate the magnitude of the two-step absorption coefficient that one 
might expect to see near the minimum of the iron absorption. We assume that three levels participate in the two-step absorption. We label these as levels 1,2 , and 3 from lowest to highest energy. The populations of these levels satisfy three simple rate equations:

$$
\begin{aligned}
& \frac{\partial N_{1}}{\partial t}=-W_{12}+N_{2} / \tau_{2} \\
& \frac{\partial N_{2}}{\partial t}=W_{12}-W_{23}-N_{2} / \tau_{2}-N_{3} / \tau_{3} \\
& \frac{\partial N_{3}}{\partial t}=W_{23}-N_{3} / \tau_{3},
\end{aligned}
$$

where $W_{12}$ and $W_{23}$ are the radiative transition rates between levels 1 and 2 and levels 2 and 3, respectively. For simplicity, radiationless relaxation has been assumed to occur only between closest states. The radiative transition rates are given approximately as follows:

$$
\begin{aligned}
& W_{12}=\left(N_{1}-N_{2}\right) \sigma_{12} I / \hbar \omega \\
& W_{23}=\left(N_{2}-N_{3}\right) \sigma_{23} I / \hbar \omega,
\end{aligned}
$$

where $\sigma_{12}$ and $\sigma_{23}$ are the transition cross-sections. We shall assume that

$$
N_{1} \gg N_{2} \gg N_{3}
$$

and

$$
\sigma_{12}=\sigma_{23}=\sigma
$$

The optical absorption coefficient $\alpha_{o p t}$ is given by

$$
\frac{d I}{d z}=\alpha_{o p t} I=\left(W_{12}+W_{23}\right) \hbar \omega
$$

or

$$
\alpha_{o p t}=\left(N_{1}+N_{2}\right) \sigma
$$

Since the ordinary linear absorption coefficient, $\alpha$, equals the product $N_{1} \sigma$, we may re-write Eq. (2.102) as

$$
\alpha_{o p t}=\alpha+N_{2} \sigma
$$


To estimate $N_{2}$, we assume that we are far from saturating the transition $1 \rightarrow 2$, and so level 2 is not filling rapidly. That is, we assume that $N_{2}$ is approximately zero, so that for pulse lengths $\Delta t$ which are short compared to the non-radiative lifetime $\tau_{2}$ we have

$$
N_{2} \approx \Delta t \frac{\partial N_{2}}{\partial t}
$$

Therefore,

$$
N_{2}=\Delta t N_{1} \sigma I / \hbar \omega
$$

We now write $\alpha_{o p t}$ as

$$
\alpha_{o p t}=\alpha+\beta I,
$$

where the effective two-photon absorption coefficient $\beta$ is given as

$$
\beta=\frac{\alpha \sigma \Delta t}{\hbar \omega}
$$

We estimate $\sigma$ by assuming an impurity concentration on the order of a few parts per million, or $N_{1}=10^{16} \mathrm{~cm}^{-3}$. Chapter 5 will show that typical values of $\alpha$ are of the order of $10^{-3} \mathrm{~cm}^{-1}$. A characteristic pulse duration of a Q-switched laser is 5 ns. For photons of $2 \mathrm{eV}$ energy, these data give

$$
\beta \approx 1 \mathrm{~cm} / \mathrm{TW} \text {. }
$$

For comparison, we now estimate an upper limit on the magnitude of the true two-photon absorption coefficient in glasses. From Eq. (2.43), we expect the maximum value of $\beta$ to scale roughly as $P E_{G}^{-3} / n^{2}$, where $P$ is an effective momentum matrix element. It is not clear what value to use for the energy gap, and so we choose a value on the order of $8 \mathrm{eV}$. Such a value is several $\mathrm{eV}$ above the energy at which glasses start to become opaque, but it is several $\mathrm{eV}$ below the level at which the intrinsic oxygen-silicon charge transfer occurs in silicate glasses, for example. ${ }^{71}$ The ratio of maximum two-photon absorption coefficient in glass to that in $\mathrm{ZnS}$ 
crystal should be approximately 1/16, counting corrections for local field effects and assuming that $P$ is the same in both materials. This means that the maximum observable two-photon absorption coefficient should be of the order of $1 \mathrm{~cm} / \mathrm{GW}$ in glasses, about three orders of magnitude higher than aciually is seen. The fact that much lower effective two-photon absorption coeficients seem to be observed indicates that, perhaps, photons of sufficiently high energy have not been used in measurements to date.

\subsection{Summary.}

In this chapter we have reviewed the formalism of two-photon absorption in solids. We emphasized the so-called perturbation-theory approach in which the two-photon absorption coefficient is determined by equating $\beta I^{2} / 2 \hbar \omega$ to the total two-photon transition rate per unit volume. We then applied the theory to the special case of zinc blende materials, which have been widely studies. The principal aim of that section was to review the results of several workers (Weiler, ${ }^{17}$ Pidgeon et al., ${ }^{18}$ Lee and Fan, ${ }^{30}$ Yee, ${ }^{31}$ and Vaidyanathan et al. $\left.{ }^{33-35}\right)$. Additionally the interesting cases of zinc blende materials with wide band gaps and narrow spin-orbit splitting was correctly formulated within the framework of perturbation theory for the first time. In the next section we briefly discussed the tunneling theory. In the subsequent section, we highlighted the nonlinear susceptibility formalism, a formalism linked strongly to classical optics and wave mixing. Since $\beta$ is proportional to the imaginary part of the third-order susceptibilty (Eq. (2.75)), ${ }^{15,58}$ the nonlinear susceptibility formalism was useful for introducing local-field effects, polarization dependence, and frequency dependence of $\beta$. Finally, we compared previous models to measured values in cubic $\mathrm{ZnS}$.

Some of the more useful results of this chapter include the correct formulation of the wide band-gap model with Kane's orbitals, the polarization-dependence 
of two-photon absorption in polycrystalline zinc blende materials, and the relationship between single-beam and dual-beam two-photon absorption. Also, it was noted several times in this chapter that for zinc blende materials, one expects $\beta$ to have an absolute maximum that scales in a known way from one material to another, namely, $\beta_{\max } \sim P / n^{2} E_{G}^{3}$, where $E_{G}$ is the band-gap energy, $P$ is an effective momentum matrix element and $n$ is the refraction index. (Pidgeon et al. ${ }^{18}$ seem to have been the first to point this out.) In addition to these results, it is hoped that the chapter can supplement the existing literature (which abounds with erroneous approximations for the two-photon âlssorption coefficient) witk a small compendium of arithmetically correct and logically consistent expressions for $\beta$ as a function of frequency for zinc blende (and diamond) structure.

The simple models used in this chapter have two limitations. First, they apply only to two-photon absorption near the band edge, and second, as discussed in Section 2.5 , they can not clearly predict the polarization- and frequency-dependence of $\beta$. We rectify these deficiencies in the next chapter. In view of the disparity between experimental results and theories that abound in the literature, some type of detailed model that can predict the dispersion of $\beta$ is necessary. 


\section{References}

1. M. Göppert-Mayer, "Über Elementarakte mit zwei Quantensprüngen," Ann. d. Phys. 9(3), 273-94 (2 May 1931).

2. V. Hughes and L. Grabner, "The radiofrequency spectrum of $R b^{85} F$ and $R b^{87} F$ by the electric resonance method," Phys. Rev. 79(2), 314-21 (15 July 1950).

3. W. Kaiser and C. G. B. Garrett, "Two-plioton excitation in $\mathrm{CaF}_{2}: \mathrm{Eu}^{2+}$," Phys. Rev. Lett. 7(6), 229-31 (15 Sept. 1961).

4. M. Inoue and Y. Toyozawa, "Two-photon absorption and energy band structure," J. Physical Soc. Jap. 20(3), 363-73 (3 Mar. 1965).

5. R. Braunstein, "Nonlinear optical effects," Phys. Rev. 123(2), 475-77 (15 Jan. 1962).

6. N. G. Basov, A. Z. Grasyuk, I. G. Zubarev, V. A. Katulin, and O. N. Krokhin, "Semiconductor quantum generator with two-photon optical excitation," Sov. Phys. JETP 23(3), 366 71 (Sept. 1966) [Zh. Eksp. Teor. Fiz. (USSR) 50, 551-59 (Mar. 1966)].

7. I. Freund, "Two-photon absorption of optical and x-ray quanta," Opt. Commun. 8(4), 401-3 (Aug. 1973).

8. A. M. Bonch-Breuvich and V. A. Khodovol̆, "Multiphoton processes," Sov. Phys. Usp. 85(1-2), 1-38 (July-Aug. 1965) [Usp. Fiz. Nauk (USSR) 85, 3-64 (Jan. 1965)].

9. J. M. Worlock, "Two-photon spectroscopy," in Laser Handbook, F. T. Arecchi and E. O. Schultz-duBois, editors (North-Holland, Amsterdam 1972).

10. V. I. Bredikhin, M. D. Galanin, and V. N. Genkin, "Two-photon absorption and spectroscopy," Sov. Phys. Usp. 16(3), 299-321 (Nov.-Dec. 1983) [Usp. Fiz. Nauk (USSR) 110, 3-43 (May 1973)]. 
11. H. Mahr, "Two-photon absorption spectroscopy," in Quantum Electronics: A Treatise, H. Rabin and C. L. Tang, editors (Academic Press, 1975).

12. Vaidya Nathan, A. H. Guenther, and S. S. Mitra, "Review of multiphoton absorption in crystalline solids," J. Opt. Soc. Am. B 2(2), 294-316 (Feb. 1985).

13. W. L. Smith, "Two-photon absorption in condensed media," in CRC Handbook of Laser Science and Technology, Volume III, M. J. Weber, editor (CRC Press, to be published).

14. L. V. Keldysh, "Ionization in the field of a strong electromagnetic wave," Sov. Phys. JETP 20(5), 1307-14 (May 1965) [Zh. Eksp. Teor. Fiz. (JSSR) 47, 1945-57 (Nov. 1964)].

15. N. Bloembergen, Nonlinear Optics (W. A. Benjamin, Inc., London, 1982).

16. C. Flytzanis, "The theory of nonlinear optical susceptibilities," in Quantum Electronics: A Treatise, H. Rabin and C. Tang, editors (Academic Press, New York, 1975).

17. M. H. Weiler, "Nonparabolicity and exciton effects in two-photon absorption in zincblende semiconductors," Solid State Comm. (Great Britain) 39(8), 937-40 (Pergamon Press, Aug. 1981).

18. C. R. Pidgeon, B. S. Wherrett, A. M. Johnston, J. Dempsey, and A. Miller, "Two-photon absorption in zinc-blende semiconductors," Phys. Rev. Lett. 42(26), 1785-88 (25 June 1979).

19. Y. Bae, J. J. Song, and Y. B. Kim, "Photoacoustic study of two-photon absorption in hexagonal ZnS," J. AppJ. Phys. 53(1), 615-19 (Jan. 1982).

20. M. L. Cohen, "Pseudopotential calculations of II-VI compounds," in II-VI Semiconducting Compounds, 1967 International Conference, D. G. Thomas, editor, 462-502 (W. A. Benjamin, Inc., NY, 1967). (See p. 480.)

21. M. D. Galanin and Z. A. Chizhikova, "Angular dependence tc two-photon ab- 
sorption in a zinc-sulfide crystal," Sov. Phys. JETP Lett. 8(10), 348-51 (20 Nov. 1968) [Zh ETF Pis. Red. (USSR) 8(10), 571-74 (20 Nov. 1968)].

22. J. H. Yee, "The two-photon transition in indirect-band gap semiconductors," J. Phys. Chem. Solids 33(3), 643-49 (Pergamon Press, March 1972).

23. L. I. Schiff, Quantum Mechanics, Third Edition (McGraw-Hill, New York, 1968).

24. R. Braunstein and N. Ockman, "Optical double-photon absorption in CdS," Phys. Rev. 134(2A), 499-507 (20 Apr. 1964).

25. D. A. Kleinman, "Laser and two-photon processes," Phys. Rev. 125(1), 87-88 (1 Jan. 1962).

26. N. W. Ashcroft and N. D. Mermin, Solid State Physics (Saunders College, Philadelphia, 1976).

27. E. O. Kane, "Band structure of indium antimonide," J. Phys. Chem. Solids 1, 249-61 (Pergamon Press, 1957).

28. G.Dresselhaus, "Spin-orbit coupling effects in zinc blende structures," Phys. Rev. 100(2), 580-86 (15 Oct. 1955).

29. R. H. Parmenter, "Symmetry properties of the energy bands of the zinc blende structure," Phys. Rev. 100(2), $573-79$ (15 Oct. 1955).

30. C. C. Lee and I. Y. Fan, "Two-photon absorption with exciton effect for degenerate valence bands," Phys. Rev. B 9(8), 3502-16 (15 Apr. 1974).

31. J. H. Yee, "Theory for two-beam two-photon photoconductivity in solids and its application to $\mathrm{ZnS}$ crystals," Phys. Rev. B 10(2), 603-6 (15 July 1974).

32. H. J. Fossum and D. B. Chang, "Two-photon excitation rate in indium antimonide," Phys. Rev. B 8(6), 2842-49 (15 Sept. 1973).

33. S. S. Mitra, L. M. Narducci, R. A. Shatas, Y. F. Tsay, and A. Vaidyanathan, “Nonlinear absorption in direct-gap semiconductors," Appl. Opt. 14(12), 303842 (Dec. 1975). 
34. A. Vaidyanathan, T. Walker, A. H. Guenther, S. S. Mitra, and L. M. Narducci, "Two-photon absorption in several direct-gap crystals," Phys. Rev. B 21(2), 743-48 (15 Jan. 1980).

35. A. Vaidyanathan, A. H. Guenther, and S. S. Mitra, "Two-photon absorption in direct-gap crystals - an addendum," Phys. Rev. B 22(12), 6480-83 (15 Dec. 1980).

36. A. Vaidyanathan, A. H. Guenther, and S. S. Mitra, "Band-structure calculations of the two-photon absorption coefficients of GaAs, InP, CdTe, and $\mathrm{ZnSe}$, Phys. Rev. B 24(4), 2259-62 (15 Aug. 1981).

37. R. Loudon, "Theory of non-linear optical processes in semiconductors and insulators," Proc. Phys. Soc. 80(Part 4, No. 516), 952-61, (1 Oct. 1962).

38. D. Boggett and R. Lundon, "Theory of exciton-polariton generation by twophoton absorption," J. Phys. C: Solid State Phys. (Great Britain) 6(10; , 176380 (24 May 1973).

39. G. D. Mahan, "Theory of two-photon spectroscopy in alkali halides," Phys. Rev. Lett. 20(7), 332 (12 Feb. 1968).

40. G. D. Mahan, "Theory of two-photon spectroscopy in solids," Phys. Rev. 170(3), 825-38 (15 June 1968).

41. M. H. Weiler, M. Reine, and B. Lax, "Theory of multiphoton magnetosorption in semiconductors," Phys. Rev. 171(3), 949-58 (15 July 1968).

42. Yu. A. Bychkov and A. M. Dykhne, "Breakdown in semiconductors in an alternating electric field," Sov. Phys. JETP 31(5), 928-32 (Nov. 1970) [Zh. Eksp. Teor. Fiz. (USSR) 58, 1734-43 (1.1ay 1970)].

43. Ya. B. Zel'dovich, "The quasi energy of a quantum mechanical system subjected to a periodic aciarn" Sov. Phys. JETP 24(5), 1006-8 (May 1967) [Zh. ETF (USSR) 51, 149?- -5 (Nov. 1967)]. 
44. H. Sambe, "Steady-states and quasienergies of a quantum-mechanical system in an oscillating field," Phys. Rev. A 7(6), 2203-13 (June 1973).

45. J. E. Bayfield, "Excited atomic and molecular states in strong electromagnetic fields," Physics Reports 51(6), 317 (1979).

46. J. C. Miklosz and R. G. Wheeler, "Exciton structure and magneto-optical effects in zinc sulfide," Phys. Rev. 153(3), 913-23 (15 Jan. 1967).

46. J. A. Armstrong, N. Bloembergen, J. Ducuing, and P.S. Pershan, "Interactions between light waves in a nonlinear dielectric," Phys. Rev. 127(6), 1918-39 (15 Sept. 1962).

47. N. Bloembergen and Y. R. Shen, "Quantum-theoretical comparison of nonlinear susceptibilities in parametric media, lasers, and I uman lasers," Phys. Rev. 133(1A), A37-49 (6 Jan. 1964).

48. P. S. Pershan, "Nonlinear optical properties of solids: energy considerations," Phys. Rev. 130(3), 919-29 (1 May 1963).

49. J. D. Jackson Classical Electrodynamics, Second Edition, (John Wiley and Sons, 1975).

50. P. N. Butcher, Nonlinear Optical Phenomena, Bulletin 200, Engineering Experiment Station (Ohio State University, 1965).

51. E. Yablonovitch, C. Flytzanis, and N. Bloembergen, "Anisotropic interference of three-wave and double two-wave frequency mixing in GaAs," Phys. Rev. Lett. 29(30), 865-68 (25 Sept. 1972).

52. R. W. Hellwarth, "Third-order optical susceptibilities of liquids and solids," in Prog. Quant. Electr. 5, 1-68 (Pergamon Press, Great Britain 1977).

53. D. Fröhlich, B. Staginnus, and S. Thurm, "Symmetry assignments of two-photon transitions in TICl," Phys. Stat. Sol. (b) 40, 287-93 (1970). 
54. T. R. Bader and A. Gold, "Polarization dependence of two-photc.1 absorption in solids," Phys. Rev. 171(3), 997-1003 (15 July 1968).

55. E. Doni, R. Girlanda, and G. Pastori Parravicini, "A note on the polarization dependence of the two-photon absorption coefficient in crystals," Phys. Stat. Sol. (b) 88(2), 773-86 (Aug. 1978).

56. W. M. McLain, "Excited-state symmetry assignment through polarized twophoton absorption studies of fluids," J. Chem. Phys. 55(6), 2789-96 (15 Sept. 1971).

57. J. F. Nye, Physical Properties of Crystals (Oxford University Press, London 1957).

58. P. D. Maker and R. W. Terhune, "Study of optical effects due to an induced polarization third order in the field strength," Phys. Rev. 137(3A), A801-18 (1 Feb. 1965).

59. N. L. Boling, Alexander J. Glass, and A. Owyoung, "Fmpirical relationships for predicting nonlinear refractive index changes in optical solids," IEEE $J$. Quantum Electr. 14(8), 601-8 (Aug 1978).

60. J. Wong and C. A. Angell, Glass Structure by Spectroscopy (Marcel Dekker, Inc., New York, 1976).

61. S. L. Adler, "Quantum theory of the dielectric constant in real solids," Phys. Rev. 126(2), 413-20 (15 Apr. 1962).

62. F. Wooten, Optical Properties of Solids (Academic Press, New York, 1972).

63. E. Panizza, "Two-photon absorption in ZnS," Appl. Phys. Lett. 10(10), 256-66 (15 May 1967).

64. K. Park and H. S. Waff, "Two-photon absorption spectrum," Phys. Lett. 28A(4), 305-6 (2 Dec. 1968).

65. E. W. Van Stryland, H. Vanherzeele, M. A. Woodall, M. J. Soileau, A. L. Smirl, 
S. Guha, and T. F. Boggess, "Two-photon absorptiori, nonlinear refraction and optical limiting in semiconductors," Opt. Engineering 24(4), 613-23 (July/Aug. 1985).

67. G. Kobbe and C. Klingshirn, "Quantitative investigation of the two-photon absorption of ruby-laser-light in various semiconductors," Z. Physik B37(1), 9-12 (1980).

68. Gmelins Handbuch der Anorganische Chemie, Zink, System-Nummer $\$ 2$ (Verlag Chemie, G. m. b. H., Weinheim, 1956).

69. J. Tauc, "Highly transparent glasses," in Optical Properties of Highly Transparent Solids, S. S. Mitra and B. Bendow, editors, 245-60 (Plenum Press, New York, 1975).

70. D. Adler, "Electronic structure of amorphous semiconductors," J. Non-Cryst. Sol. 42, 315-34 (North Holland, Amsterdam, 1980).

71. S. T. Pantelides, "The optical absorption spectrum of $\mathrm{SiO}_{2}$," in The Physics of $\mathrm{SiO}_{2}$ and its Interfaces, S. T. Pantelides, editor, 80-84 (Pergamon Press, New York, 1978). 


\section{Chapter 3}

\section{PSEUDOPOTENTIAL COMPUTATION OF TWO-PHOTON}

\section{ABSORPTION IN ZINC BLENDE}

\subsection{Introduction.}

This chapter discusses the pseudopotential calculation of two-photon absorption in four steps. Section 3.1 discusses the general one-electron Bloch formalism. In this formalism, the Schrödinger Wave Equation is replaced by an equivalent determinantal equation. Section 3.2 discusses the form of the pseudopotential itself. That section is based extensively on work by Heine and Cohen. ${ }^{1}$ Section 3.3 discusses the band structure of a specific material, cubic zinc sulfide and compares the results to previous work. Section 3.4 presents the two-photon absorption calculation.

In order to compute the optical two-photon absorption coefficients quantum mechanically for a material, one must first determine the electronic wave functions and eigenenergies of that material. Some techniques of estimating electronic states of solids include ${ }^{2}$ the empty lattice (plane wave) method, the orthogonal plane wave method, the augmented plane wave method, tight binding methods, and pseudopotential methods. Each technique has its own merits, but empirical pseudopotential methods have been use more than all other methods combined to compute electronic band structures of semiconductors. ${ }^{1,3}$ The results have been almost spectacular. In some cases when measurements have disagreed with pseudopotential predictions, the measurements, not the predictions, were subsequently shown to be incorrect. ${ }^{3}$

Despite wide use in modeling linear optical properties, pseudopotentials have only recently been used to compute nonlinear optical absorption. Vaidyanathan and co-workers have reported calculating $\beta$ in several zinc blende materials (GaAs, 
InP, CdTe, and $\mathrm{ZnSe}$ ) at various harmonics of the Nd laser line., ${ }^{4,5}$ They apparently used Cohen and Bergstresser's ${ }^{6}$ pseudopotential form factors. ${ }^{5}$ Other application of pseudopotentials to intrinsic optical nonlinearities in solids have been Fong and Shen's computation of the second-order susceptibility $\chi^{(2)}$ in $\mathrm{GaAs}$, InAs, and $\operatorname{InSb}^{7}$ and Matynov and Rozenson's calculation of $\chi^{(2)}$ in $\mathrm{HgGa}_{2} \mathrm{~S}_{4} .^{8}$

In the empirical pseudopotential method, an operator, the "pseudopotential," replaces the potential energy function of the one-electron Schrödinger Wave Equation. The pseudopotential is chosen so that the eigenvalues of the modified wave equation (the "pseudo wave equation") reproduce the energy gaps of the solid at selected points of high symmetry in the Brillouin Zone. Additionally, one may demand that the solutions of the pseudo wave equation form an accurate basis for computing some other observable feature of the solid, such as the linear dielectric tensor. One then iterates between adjusting the pseudopotential parameters and computing the desired physical quantities until satisfactory agreement is reached between experiment and theory. At that point the pseudopotential is completely defined, and it can be used for calculating other physical quantities., ${ }^{1,3}$

\subsection{Determinantal Form of the One-Electron Wave Equation.}

The quantum state of a system of interacting non-relativistic particles satisfies a many-body Schrödinger equation. Since no closed solution of the many-body problem is presently known, the problem is solved by successive approximations. To first order, all multi-particle correlations are disregarded, and each particle is viewed as independent of all the remaining particles except for an effective bulk potential energy term. When the particles are electrons, this is the "independent electron" approximation.

In a crystal, the innermost electrons around each nucleus are generally bound tightly enough to regard the solid as a set of "core" ions plus a set of outer "valence" 
electrons that provide over-all electrical neutrality and bind the cores together. The positions of the core ions are taken as fixed in some configuration, such as the mean $T=0 \mathrm{~K}$ arrangement. Due to the tight binding of the core electrons and the large masses of the nuclei, only the valence electrons are regarded as optically active.

Structurally, the crystal is treated as a set of identical unit cells arrayed on a geometrically regular lattice of points. A unit cell contains one or more atomic cores. Neglecting edge effects at the boundaries of the crystal, one imagines all of space to be spanned by an infinite set of identical, contiguous crystals. Because of the assumptions of translational periodicity of the unit cells and of Born-von Kármán periodic boundary conditions, both the potential energy and the solutions to the time-independent one-electron Schrödinger Wave Equation may be expressed as discrete Fourier sums of plane waves. ${ }^{2}$

Taking the inner product between the Schrödinger Wave Equation and every possible plane wave basis function $\exp (i \vec{G} \cdot \vec{r})$ leads to an infinite-order matrix eigenvalue problem, the secular equation of which is Bloch's determinantal form of Schrödinger's Wave Equation (Appendix A):

$$
0=\operatorname{det}\left[\left(\frac{\hbar^{2}|\vec{k}-\vec{G}|^{2}}{2 m}-E\right) \delta_{\vec{G} \vec{G}^{\prime}}+\sum_{j} V_{j}\left(\vec{G}, \vec{G}^{\prime}\right) S_{j}\left(\vec{G}-\vec{G}^{\prime}\right)\right]
$$

The quantity in square brackets is an infinite Hermitian matrix whose rows and columns are indexed by the reciprocal lattice vectors $\vec{G}$ and $\vec{G}^{\prime}$. The wave vector $\vec{k}$ is restricted to the first Brillouin zone. The constants $m$ and $\hbar$ are respectively the free-electron mass and Planck's constant. The symbol $\delta_{\vec{G} \vec{G}^{\prime}}$ is a Kronecker delta function, and $E$ is the energy eigenvalue, which depends upon $\vec{k} . V_{j}\left(\vec{G}, \vec{G}^{\prime}\right)$ is the atomic form factor of the $\mathrm{j}$-th type of atom in a unit cell. It is defined as

$$
V_{j}\left(\vec{G}, \vec{G}^{\prime}\right)=\frac{n_{j}}{\Omega} \int_{-\infty}^{\infty} d^{3} \vec{r} \exp (-i \vec{G} \cdot \vec{r}) U_{j}(\vec{r}) \exp \left(i \vec{G}^{\prime} \cdot \vec{r}\right)
$$


where $U_{j}(\vec{r})$ is the effective atomic potential of an atom in the crystal, $n_{j}$ is the number of type $\mathrm{j}$ atoms per unir cell, and $\Omega$ is the volume of a unit cell. The quantity $S_{j}\left(\vec{G}-\vec{G}^{\prime}\right)$ is the structure factor of the j-th type of atom. It is defined as

$$
S_{j}\left(\vec{G}-\vec{G}^{\prime}\right)=\frac{1}{n_{j}} \sum_{\vec{\rho}_{j}} \exp \left(i\left(\vec{G}-\vec{G}^{\prime}\right) \cdot \vec{\rho}_{j}\right)
$$

where $\vec{\rho}_{j}$ is the position of one type-j atom in a unit cell.

Given the structure of a crystal and a complete set of form factors $V_{j}\left(\vec{G}, \vec{G}^{\prime}\right)$, it is conceptually straight-forward to compute the eigenvalues and wave functions that satisfy Schrōdinger's Equation. First, the eigenvalues, or energy bands, $E(\vec{k})$ are obtained from Eq. (3.1). Then the wave functions are determined for every separate value of $\vec{k}$ and $E(\vec{k})$ by solving a matrix eigenvalue equation of the following form:

$$
\begin{aligned}
H_{\vec{G} \vec{G}^{\prime}} C_{\vec{G}^{\prime}} & =E(\vec{k}) C_{\vec{G}}, \\
H_{\vec{G} \vec{G}^{\prime}} & =\frac{\hbar^{2}}{2 m}|\vec{k}-\vec{G}|^{2} \delta_{\vec{G} \vec{G}^{\prime}}+\sum_{j} V_{j}\left(\vec{G}, \vec{G}^{\prime}\right) S\left(\vec{G}-\vec{G}^{\prime}\right)
\end{aligned}
$$

The eigenfunction for a given wave vector $\vec{k}$ and a given energy band $E$ is then a superposition of an infinite number of plane waves:

$$
\Psi_{E(\vec{k})}=\sum_{\vec{G}} C_{\vec{G}} \exp (i(\vec{k}-\vec{G}) \cdot \vec{r})
$$

For computational purposes, the series in Eq. (3.6) must be limited to a finite number of terms.

3.2 The Local Pseudopotential Approximation with Spherical Form Factors.

As discussed in References 1 and 3, many plane wave components are required to solve Eq. (3.6) accurately when $U_{j}$ is the "true" one-electron potential, because the true valence wave functions oscillate rapidly with position near ionic cores. The fundamental reason for this is, valence electrons have more total energy than core electrons. Higher total energy means higher kinetic energy, which is represented in 
the Schrödinger Equation by higher curvature of the wave function. Near the core, where the potential energy is lowest, electrons have the greatest kinetic energy, and so undulations are most rapid near the core.

The chief approximation of the pseudopotential method is to replace the deep, attractive "true" potential that belongs in Eq. (3.2) with a pseudopotential operator $V_{j}^{p s}$ which is as shallow and smooth as possible inside the core. The fact that the valence electrons spend little time within the core justifies neglecting their detailed behavior in the core. ${ }^{1,3}$ This pseudopotential, with its "filled in" well near each core, gives a wave equation whose solutions have low effective kinetic energy and hence low curvature within the core regions. Outside the core, the pseudopotential is identical to the original potential $U_{j}$. An accurate calculation using a "true" screened Coulomb potential would require many thousands of plane waves, even for a relatively light atom like aluminum, but a typical pseudopotential calculation uses only 50 to 100 plane wave components. ${ }^{1}$ Solving the wave equation reduces to a problem of finding the eigenvalues and eigenvectors of a $50 \times 50$ or $100 \times 100$ matrix, which is within the capabilities of today's computers.

To simplify the calculation of eigenvalues and eigenfunctions, one assumes that the potential energy wells of the ionic cores are spherically symmetric. This is rigorously accurate only for isolated atoms, but it often yields good results even in covalently bonded materials among the groups IV, III-V, and II-VI semiconductors, which includes $\mathrm{ZnS}$. In this approximation the form factors of the pseudopotential depend only upon the magnitude of the wave vector $\vec{G}-\vec{G}^{\prime}$. That is,

$$
V_{j}\left(\vec{G}, \vec{G}^{\prime}\right)=V_{j}\left(\left|\vec{G}-\vec{G}^{\prime}\right|\right)
$$

In Eq. (3.7), we have omitted the superscript "ps." With spherical atomic form factors, directionality in the covalent bonds is retained through the exponential phase functions in the structure factor. 
A goal of the pseudopotential method is transferability. It is desirable to find a characteristic pseudopotential for every atomic element so that, by scaling and mixing pseudopotentials of different elements, one can obtain a pseudopotential for any chemical compound. From a set of form factors $V_{j}^{A}\left(\vec{G}, \vec{G}^{\prime}\right)$ for element $\mathrm{j}$ in compound $\mathrm{A}$, the form factors $V_{j}^{B}\left(\vec{G}, \vec{G}^{\prime}\right)$ for the same element in a different compound $\mathrm{B}$ are approximately given by ${ }^{1}$

$$
V_{j}^{B}\left(\vec{G}, \vec{G}^{\prime}\right)=\frac{\varepsilon^{A}}{\varepsilon^{B}} \frac{n_{j}^{B} / \Omega^{B}}{n_{j}^{A} / \Omega^{A}} V_{j}^{A}\left(\vec{G}, \vec{G}^{\prime}\right) .
$$

The ratio of the static linear dielectric constants $\varepsilon^{A} / \varepsilon^{B}$ accounts for the lowestorder variations in electronic screening in the two compounds. The ratios $n_{j}^{A} / \Omega^{A}$ and $n_{j}^{B} / \Omega^{B}$ account for differences between the number densities (atoms $/ \mathrm{cm}^{3}$ ) of element $j$ in compounds $A$ and B, respectively. Note that Eq. (3.8) assumes that the magnitudes of the reciprocal lattice vectors $\vec{G}$ and $\vec{G}^{\prime}$ are unchanged in compounds A and B. Since A and B may have different structures or lattice spacings, it becomes necessary to interpolate the pseudopotential between values of the reciprocal lattice vectors. This can easily be done if the pseudopotential is a known continuous function of the parameter $\left|\vec{G}-\vec{G}^{\prime}\right|$. Falicov and Lin have introduced the following approximation for atomic form factors: ${ }^{9}$

$$
V(q)=\left\{\begin{array}{cc}
A_{1}\left(q^{2}-A_{2}\right) /\left(1+\exp \left(A_{3}\left(q^{2}-A_{4}\right)\right)\right), & q \leq q_{c} \\
0, & q>q_{c}
\end{array}\right.
$$

where the dimensionless wave vector $q$ is defined so that

$$
q^{2}=\frac{1}{2}\left|\frac{\vec{G}-\vec{G}^{\prime}}{k^{F}}\right|^{2}
$$

The parameter $k^{F}$ is the effective radius of the Fermi sphere in a crystal of the pure substance j. That is, $\left(\hbar k^{F}\right)^{2} / 2 m=E_{F}$ where $E_{F}$ is the Fermi energy. The cutoff wave vector $g_{c}$ is a disposable parameter whose value is chosen to limit the expansions in Eqs. (3.1), (3.5), and (3.6) to a manageable number of terms-usually to less than seven distinct magnitudes of $\left|\vec{G}-\vec{G}^{\prime}\right|$. 
The preceeding desidiratum, having a unique transferable pseudopotential for each atomic element, cannot be met. First, pseudopotentials are never unique. As shown by Cohen and Heine ${ }^{1}$, many different pseudopotentials can be constructed which cause a pseudo wave equation to preserve the eigenvalues of a given original wave equation. Furthermore, a pseudopotential is generally an integral operator which is both non-local and eigenenergy-dependent. 1,3

Despite the non-local and eigenenergy-dependent nature of pseudopotentials, often a local (i.e., scalar), energy-independent function with spherically symmetric atomic form factors can be used as a pseudopotential. There is no rigorous a priori justification for expecting a scalar function to give accurate band structures, as has been discussed elsewhere. ${ }^{3}$ In the final analysis, the choice of a local or non-local operator is justified by its successful results.

\subsection{Pseudopotential Calculations of Energy Bands in Cubic ZnS.}

Cohen and Bergstresiar have successfully used the local empirical pseudopotential method to compute electronic band structure of $\mathrm{ZnS}$.,10 The form factors have been reported along with the resulting band structure. The valence electrons in zinc and sulfur are s- and p-orbitals. Although zinc has d-electrons, they fill the $d$-shell and are optically inert over the photon energy range of interest in this work. Some researchers have attempted to improve upon the local pseudopotential model used by Cohen and Bergstresser by adding non-local corrections to the pseudopotential, ${ }^{11,12}$ but the empirical local pseudopotential gave better agreement between theoretical and measured optical properties of $\mathrm{ZnS}$ than did the non-local pseudopotential. Therefore, the local pseudopotential will be used in this paper. Other workers have attempted to compute an accurate self-consistent pseudopotential, ${ }^{13}$ but Cohen and Bergstresser's empirical appro .ch is at least twice as accurate in $\mathrm{ZnS}$. 
Cubic zinc sulfide has a zinc blende structure. The ion sites form two identical parallel face centered cubic (fcc) lattices displaced by one quarter of a body diagonal. One lattice has only zinc ions on it; the other, only sulfur. We choose the center of each unit cell to lie halfway between adjacent zinc and sulfur ions so that each unit cell contains a zinc ion at $(1,1,1) \mathrm{b} / 8$ and a sulfur ion at $-(1,1,1) \mathrm{b} / 8$, where $b=5.42 \AA$ is the length of a side of one face-centered cube. ${ }^{6}$

We take the pseudopotential to have the form of Eq. (3.9) as suggested by Lin and Falicov. ${ }^{9}$ Figure 3-1 shows the pseudopotentials of zinc and sulfur as functions of the dimensionless wave vector q. From the solid curve we obtained the pseudopotential form iactors that we used in this work. For comparison, tabulated values of $\left(\Omega^{A} / \Omega^{Z n S}\right) V_{Z n}$ and $\left(\Omega^{A} / \Omega^{Z n S}\right) V_{S}$ ("A" $=\mathrm{ZnS}, \mathrm{ZnSe}, \mathrm{ZnTe}$, and CdS) are superimposed on curves, as in Reference 1. Substituting the pseudopotential form factor $V_{j}$ defined by Eq. (3.9) in place of the true form factors $V_{j}$ in Eqs. (3.1), (3.5), and (3.6) gives the energy levels and the plane wave representation of the pseudo wave functions for a set of 648 wave vectors $\vec{k}$ uniformly spaced through-out the irreducible Brillouin zone. (See Appendix C.) The irreducible Brillouin zone is the smallest segment of the Brillouin zone that covers the complete BZ when every element of the point group operates on it.

A Fortran pseudopotential program written by others, JOCK35TW, was used with only slight modifications to compute the solutions to the pseudo wave equation. A packaged matrix solver subroutine returned the eigenvalues $E$ and the plane wave coefficients $C_{\vec{G}}$. The wave functions returned by the subroutine were orthonormal. That is,

$$
\sum_{\vec{G}} C_{\vec{G}}^{*} C_{\vec{G}^{\prime}}=\delta_{E(\vec{k}), E^{\prime}(\vec{k})}
$$

The plane wave expansions were truncated at $|G|^{2}=12(2 \pi / b)^{2}$. The number of terms was therefore 59. (See Appendix B.) Increasing the number of terms did not 


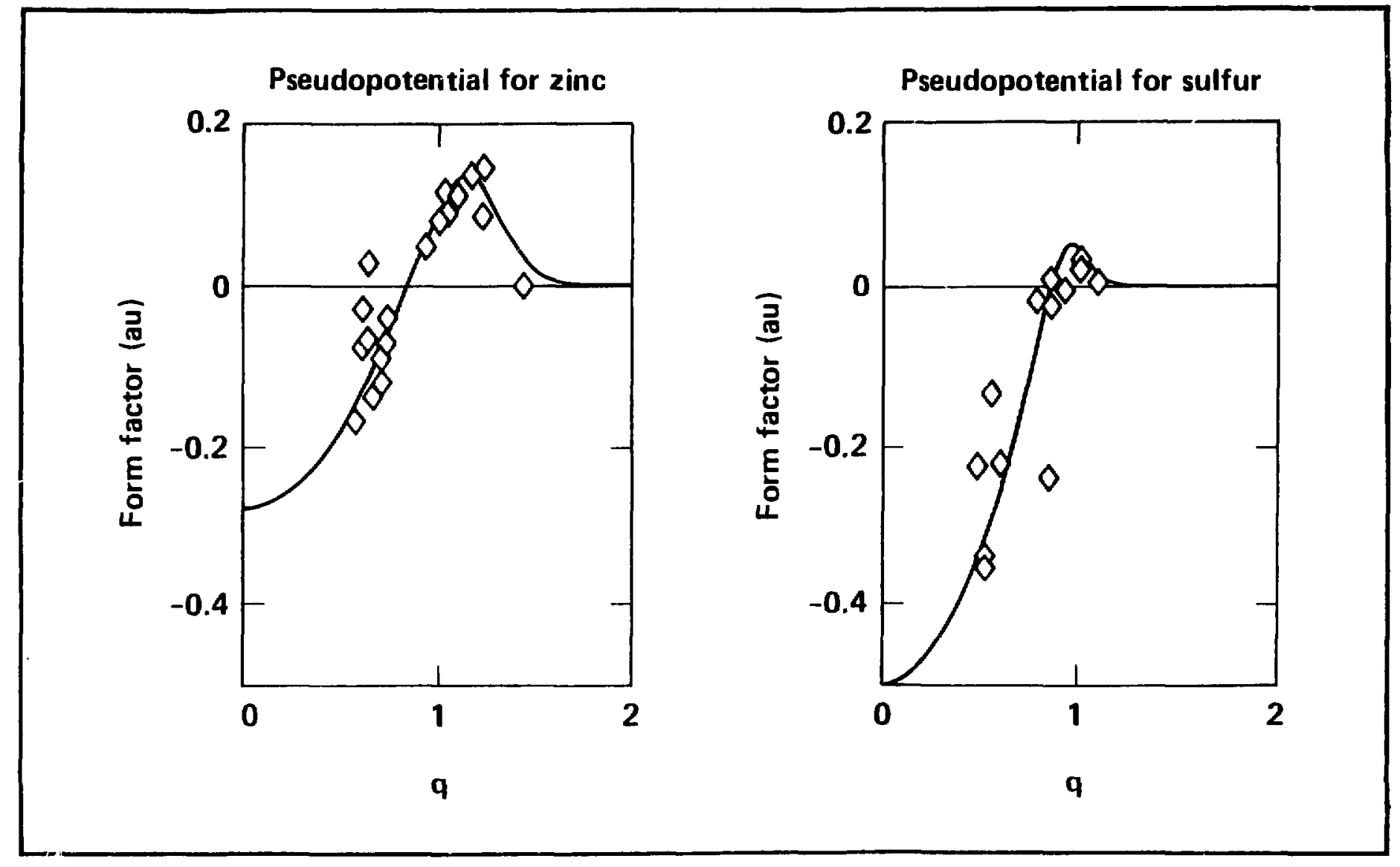

Figure 3-1. Pseudopotential Form Factors for Zinc and Sulfur. The diamonds are values of the form factoris th:t others have previously compuled.' The curves are the functions used in this work. 
significantly change the computed band structure. The lowest energy bands (four valence and six conduction) were compared to Cohen and Bergstresser's results ${ }^{6}$ and then stored together with the coefficients $C_{\vec{G}}$ for later use in computing the optical properties of $\mathrm{ZnS}$. Table (3-1) gives the parameters used in JOCK35TW.

Figure 3-2 shows the comparison of our calculations to Cohen and Bergstesser's results. Between the two theories, the $0 \mathrm{~K}$ band-gap energies are the same, and so are the lowest conduction-band and highest valence band. There is slight disagreement for some of the bands. These slight differences will prove to be unimportant in computing optical properties, showing that the dominant contributions to optical properties arise from the energy bands closest to the band gap. In conputing the band structure for $\mathrm{ZnS}$, we have neglected spin-orbit coupling.

As a check, we computed the linear optical properties of cubic $\mathrm{ZnS}$, following Cohen, ${ }^{1,6}$ and Brust. ${ }^{14}$ First, we determined the imaginary part of the linear dielectric tensor, $\epsilon^{\prime \prime}(\omega)$. For cubic materials, it is

$$
\varepsilon^{\prime \prime}(\omega)=\frac{4 \pi^{2} e^{2} \hbar}{3 m \omega^{2}} \sum_{n, s} \int_{B Z} \frac{2}{(2 \pi)^{3}} \delta\left(\frac{E_{n}(\vec{k})-E_{s}(\vec{k})}{\hbar}-\omega\right)\left|M_{n s}^{(1)}(\vec{k})\right|^{2} d^{3} \vec{k}
$$

where

$$
M_{n s}^{(1)}(\vec{k})=\left\langle\psi_{E_{n}(\vec{k})}|\vec{\nabla}| \psi_{E_{\mathrm{r}}(\vec{k})}\right\rangle
$$

The subscripts $n$ and $s$ denote the filled and empty bands, respectively.

Figure 3-3 plots the mean value of $\varepsilon^{\prime \prime}$ averaged over steps $\Delta \omega=2 \times 10^{14} \mathrm{~Hz}$ $(0.14 \mathrm{eV})$.

$$
\begin{aligned}
\vec{\varepsilon}^{\prime \prime}(\omega) & =\frac{1}{\Delta \omega} \int_{\omega-\Delta \omega / 2}^{\omega+\Delta \omega / 2} \varepsilon^{\prime \prime}\left(\omega^{\prime}\right) d \omega^{\prime} \\
& =\frac{e^{2} \hbar}{3 m \omega^{2} \triangle \omega} \sum_{n, s} \int_{B Z} d^{3} \vec{k} \\
& \times H\left(\frac{E_{B}(\vec{k})-E_{n}(\vec{k})-\hbar \omega}{\hbar \Delta \omega}\right)\left|M_{n s}^{(1)}(\vec{k})\right|^{2}
\end{aligned}
$$




\begin{tabular}{|l|ccc|}
\hline Number of bands $\quad 59$ & & & \\
Lattice constant $(\AA)^{a} \quad b=5.42$ & & & \\
& & & \\
& Zinc & Sulfur & ZnS \\
& & & \\
\hline & & & \\
Effective Unit Cell Volume, $\Omega$ (au) & 102 & 178 & 133 \\
Fermi Wavevector, $k_{F}$ (au) & 0.8334 & 1.02 & \\
Cutoff Wavevector, $q_{c}$ (dimensionless) & 1.8 & 1.25 & \\
Pseudopotential Form Factors & & & \\
C1 (Energy, au) & 0.4 & 0.67 & \\
C2 (dimensionless) & 0.7 & 0.75 & \\
C3 (dimensionless) & 4.0 & 10.0 & \\
C4 (dimensionless) & 1.37 & 0.9 & \\
& & & \\
\hline
\end{tabular}

Table 3-1. Parameters used to Compute the Pseudopotential of Cubic $Z_{n} S$

"Ref. 6 


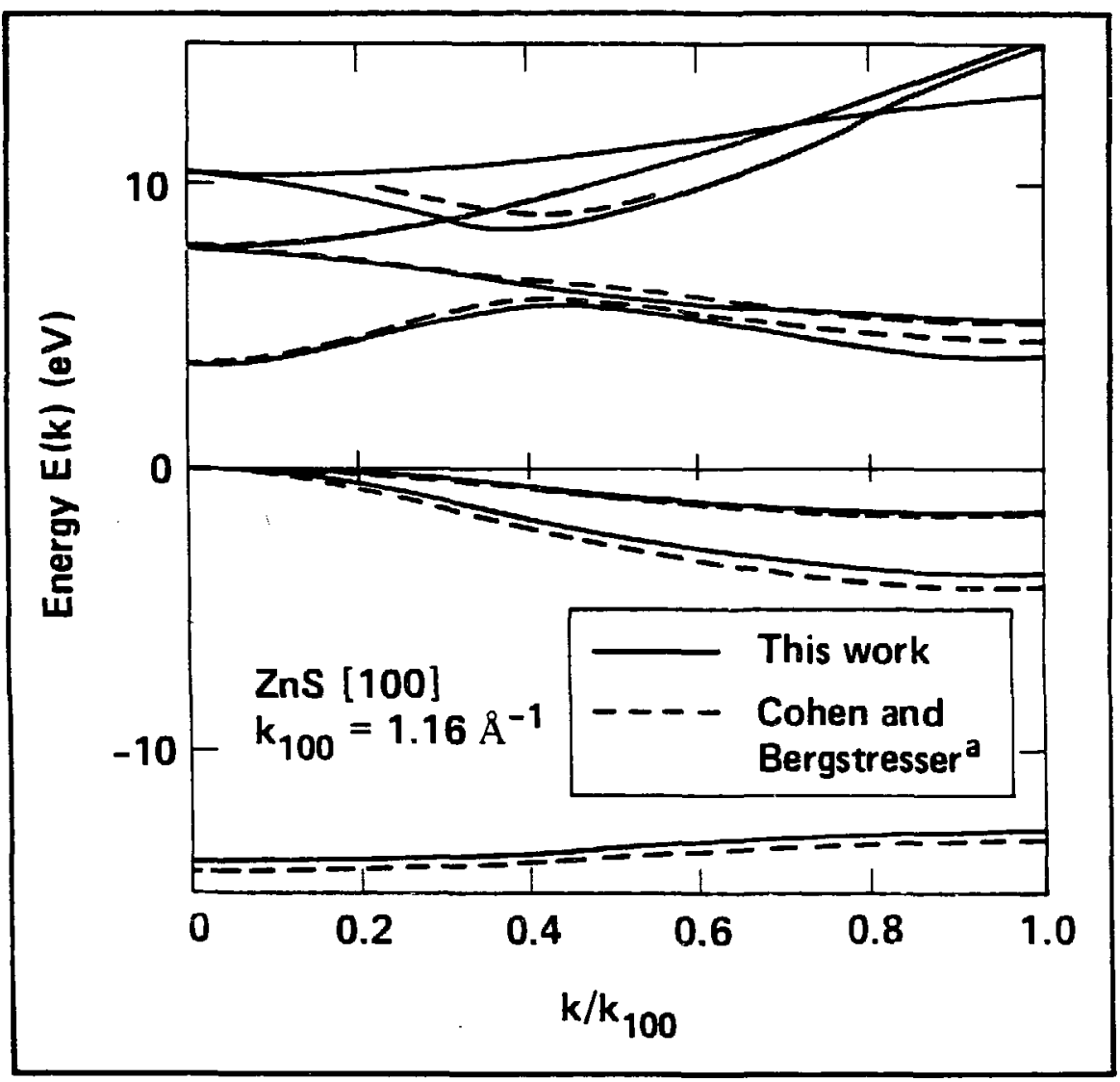

Figure 3-2. Energy Bands along [100] in Cubic $\mathrm{ZnS}$. The pseudopotential method that was used was similar to that of Cohen and Bergstresser. ${ }^{6}$ Only the lowest ten eigenvalues are shown; fifty-nine were computed. Spin was neglected. Energies are referenced to zero at the top of the valence bands. 
where

$$
H(x)= \begin{cases}1, & |x|<\frac{1}{2} \\ 0, & \text { otherwise }\end{cases}
$$

From a Kramers-Krönig transformation ${ }^{15}$ we obtained the real part of the dielectric tensor, $\varepsilon^{\prime}(\omega)$ :

$$
\varepsilon^{\prime}(\omega)=1+\frac{2}{\pi} \oint_{0}^{\infty} \frac{\omega^{\prime} \varepsilon^{\prime \prime}\left(\omega^{\prime}\right) d \omega^{\prime}}{\left(\omega^{\prime}\right)^{2}-\omega^{2}}
$$

The circle in the integral sign implies that the Cauchy principal value must be taken.

From $\varepsilon^{\prime}$ and $\varepsilon^{\prime \prime}$ the small angle reflectance $R(\omega)$ was computed.

$$
R=\left|\frac{\sqrt{\varepsilon}-1}{\sqrt{\varepsilon}+i}\right|^{2},
$$

where

$$
\varepsilon=\varepsilon^{\prime}+i \varepsilon^{\prime \prime}
$$

Figure 3-4 compares our results to previous experimental and theoretical results, showing generally good agreement with previous work up to photon energies between 7 and $8 \mathrm{eV}$. Beyond $8 \mathrm{eV}$, the measured reflectances are much lower than the values computed with our pseudopotential. Since the core states are believed to lie over $14 \mathrm{eV}$ below the lowest conduction band, ${ }^{6}$ it seems unlikely that core states account for the discrepancy. Rather, it is more likely that the energy bands are slightly erroneous. Since we are interested in two-photon transitions less than $7.4 \mathrm{eV}$ (twice the band-gap energy), we do not attempt to improve upon the poor refiectance calculation at high photon energies.

Two aspects of Figure 3-4 deserve special mention. First, a small doublet appears in the vicinity of $\hbar \omega=4 \mathrm{eV}$. This doublet has not been predicted in previous pseudopotential calculations. Its appearance in this work is due strictly to the way that the irreducible Brillouin zone was sampled in the summation over all $\vec{k}$ in Eq. 


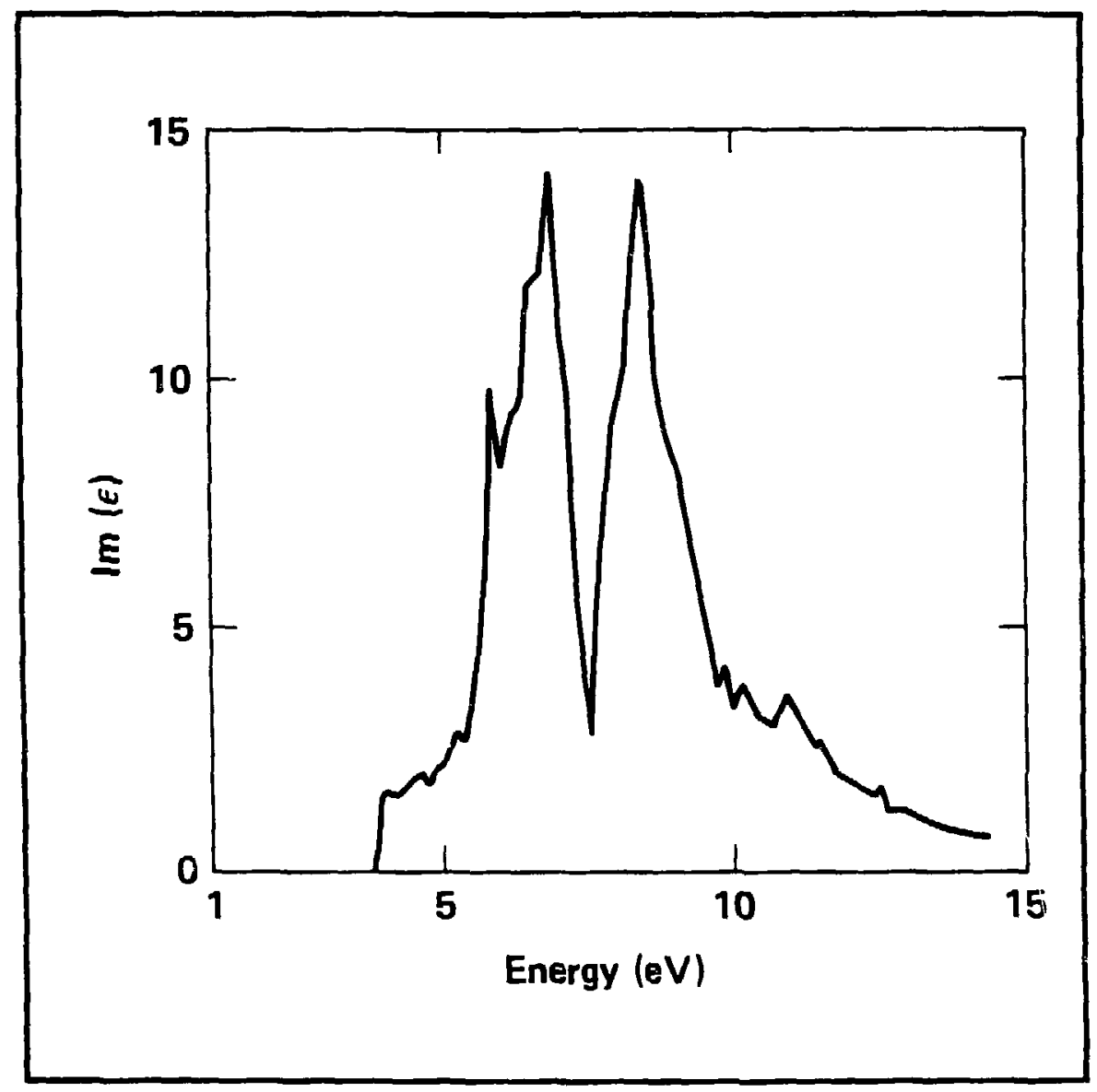

Figure 3-3. Imaginary Part of the Linear Dielectric Constant, $\epsilon$ " or $\operatorname{Im}\left(\chi^{(1)}\right)$. The results, computed after Brust, ${ }^{14}$ agree favorably with previous calculations by Cohen et al. ${ }^{1,6}$ 


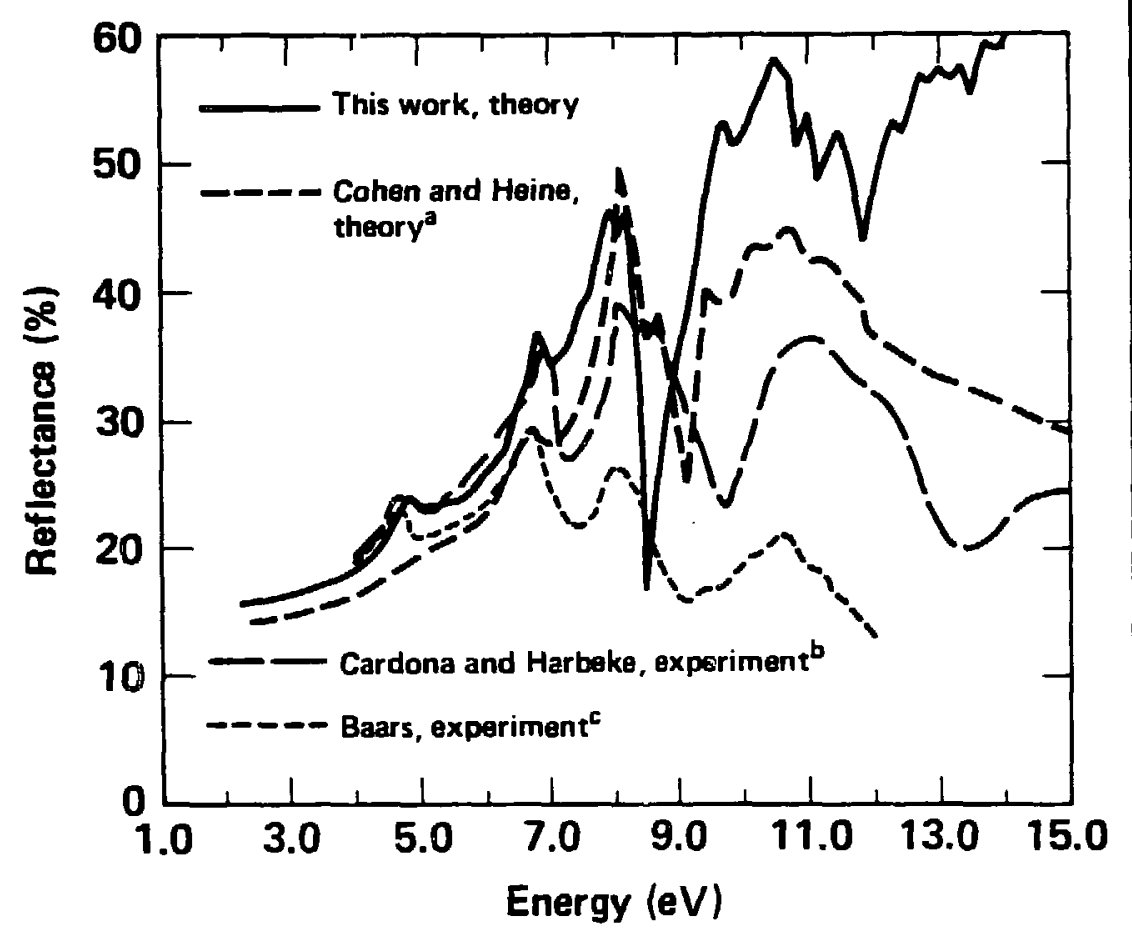

Figure 3-4. Optical Reflectance of $\mathrm{ZnS}$, Computed from the Dielectric Constant Shown in the Preceding Figure. Note the small spike that occurs just above $5 \mathrm{eV}$ in the experimental results. This spectral feature is absent from the results of Cohen et al., ${ }^{1,6}$ but it is predicted by our model.

"Ref. 1

${ }^{b}$ Ref. 16

c Ref. 17 
(3.12). (See Section 3.4.1 and Appendix C.) Second, there are no adjustable parameters in the computation of $\varepsilon^{\prime \prime}$ apart from the parameters in the pseudopotential itself. These facts illustrate the obvious, that using the empirical pseudopotential method requires careful modeling of more than just band structure parameters. It also requires careful modeling of physically observable properties like optical reflectance. Finally, the generally good agreement between our calculation and experimental results suggests that the pseudopotential that we shall use to compute two-photon absorption in the next section is probably adequate as long as $\hbar \omega$ does not exceed $E_{G}$.

\subsection{Calculation of Two-Photon Absorption in ZnS.}

Figure 3-5 shows the two-photon absorption spectra that we computed for several polarizations in cubic $\mathrm{ZnS}$ at $0 \mathrm{~K}$. The curves were smoothed twice with a three-point running average. For two-photon energies above $6.6 \mathrm{eV}$, we obtained large spikes in the data, indicating possible singularjties. Based on experience in calculating the linear absorption spectrum of $\mathrm{ZnS}$, we believe that the spikes are isolated numerical artifacts that can be corrected by changing the step size in the integral over the Brillouin zone (BZ). We have not attempted to correct them because our predictions already extend to $6.6 \mathrm{eV}$, which is about $1.0 \mathrm{eV}$ farther than any available measurements. Moreover, agreement between experiment and theory is quite good, as Figure 3-6 shows.

The experimental data in Figure 3-6 were obtained at room temperature, as discussed in Section 2.6. To simu'ate the effect of temperature, we translated our $0 \mathrm{~K}$ predictions to lower energies. No special attempt was made to align our pseudopotential predictions with the experimental curves so that the figure would appear nice. We simply translated the theoretical curves by $-0.18 \mathrm{eV}$, which is the difference between $E_{G}$ for $\mathrm{ZnS}$ at room temperature $\left(3.66 \mathrm{eV}^{18}\right)$ and $E_{G}$ at 0 


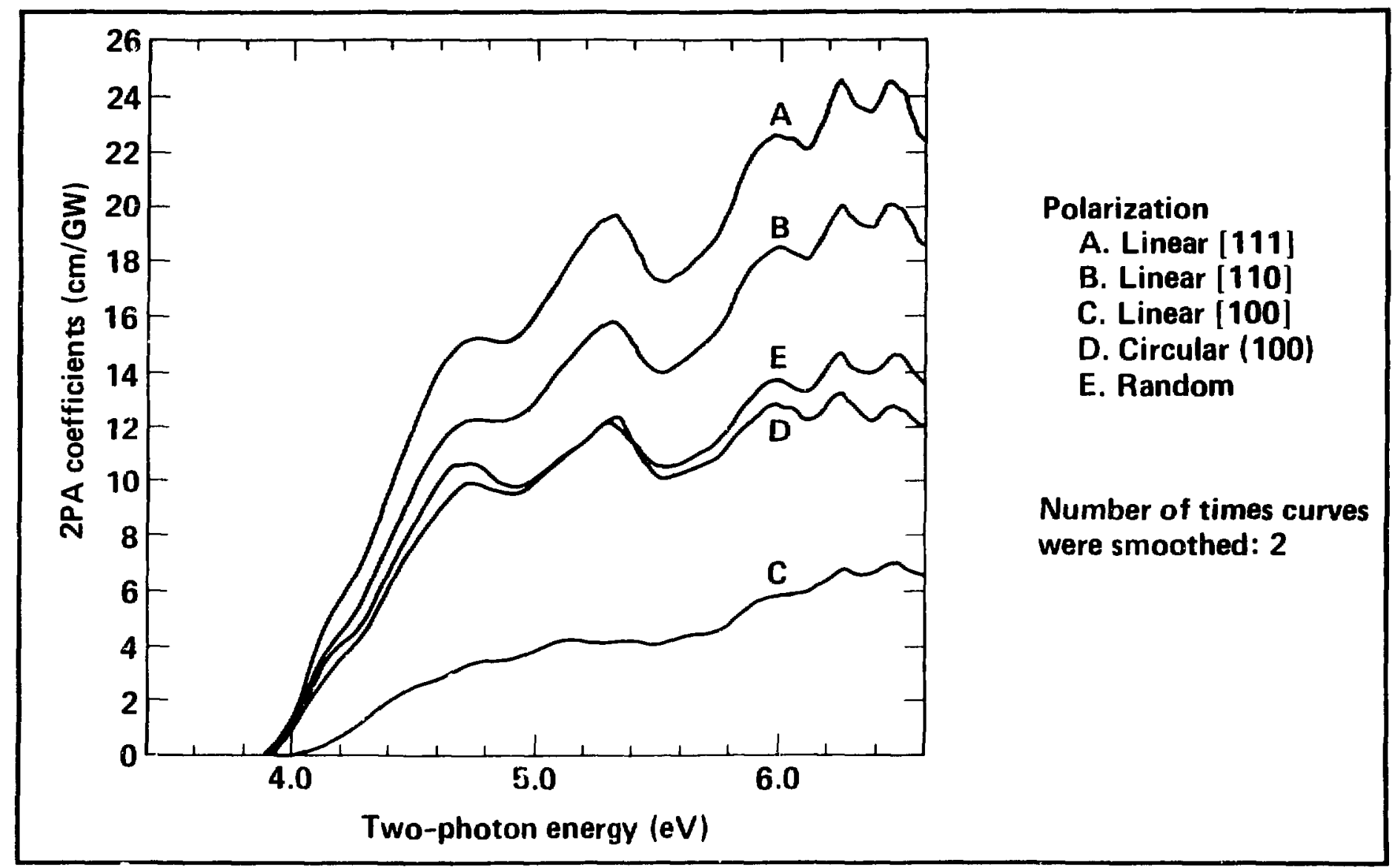

Figure 3-5. Pscudopotential Calculation of $\beta$. The various curves are for different directions of polarization. 


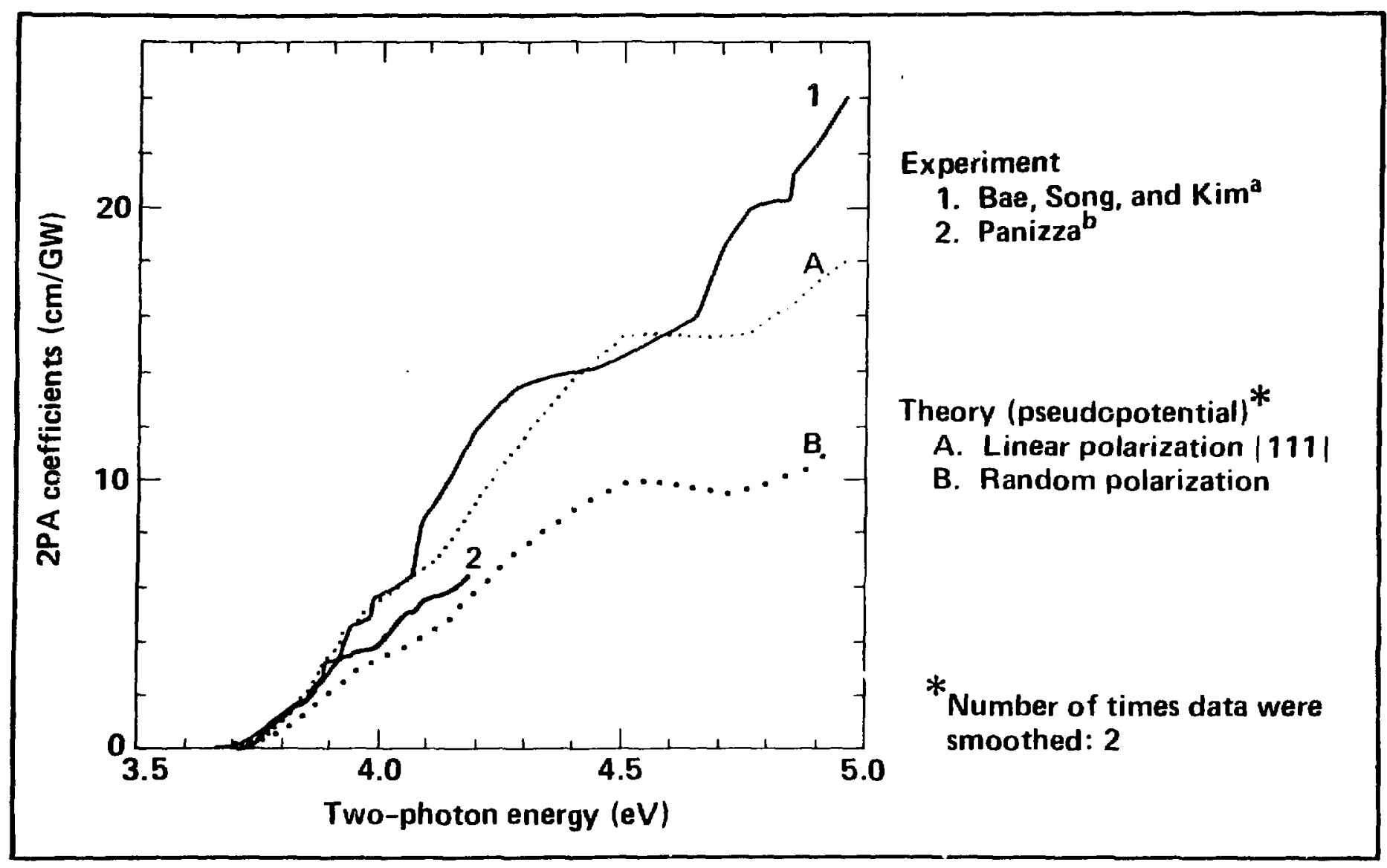

Figure 3-6. Comparison of Experiment and Pseudopotential Valuns of $\beta$.

"Rof. 11" "Ref. 22 
$\mathrm{K}\left(3.84 \mathrm{eV}^{10}\right)$. Also, no adjustable scale factors were used in the theoretical resuit. Once we adjusted the empirical pseudopotential parameters to fit the linear spectrum of $\mathrm{ZnS}$, we computed two-photon absorption without modifying the form factors. Figure 3-6 is significant because the pseudopotential calculation not only agrees closely in magnitude with experimental spectra over a wide range (especially the data of Bae, Song, and $\mathrm{Kim}^{19}$ ), but it agrees in detailed shape, too. This is due to using more exact wave functions than Kane's simple theory admits and to having excellent experimental data available. For comparison, one should use the value of $\beta$ computed along the 111-axis of zinc blende to the value of $\beta$ measured the cylindrical axis of v. urtzite. The fact that the experiment and theory agree so well-especially near the absorption edge, suggests that Cohen ${ }^{1,20}$ and Galanin and Chizhikova ${ }^{21}$ were correct in stating that the hexagonal and cubic phases of $\mathrm{ZnS}$ should yield similar spectra for light polarized along the cubic (111) and the hexagonal (001) axes. It also tends to confirm both our pseudopotential model and the experimental data of Bae et al.

The remainder of this section is devoted to clarifying how we computed the two-photon absorption spectra and to drawing a few conclusions about the various theoretical methods that have been discussed in Chapter 2 and 3.

\subsubsection{Mechanics of Calculating $\beta(\omega)$.}

This section outlines the mathematics behind the computation of $\beta$. From Eq. (2.9), the total two-photon absorption coefficient $\beta$ in an insulator is a set of individual two-photon atsorption coefficients $\beta_{c v}$ summed over all conduction bands $c$ and valence bands $v$. Each term $\beta_{c v}$ is of the form

$$
\begin{aligned}
\beta_{c v}(\omega)(\mathrm{cm} / \mathrm{GW}) & =2 \times 10^{16} \frac{\omega}{(n c)^{2}}\left(\frac{e}{m \omega}\right)^{4} \\
& \times \int_{B Z} d^{3} \vec{k} \delta\left(E_{c v}(\vec{k})-2 \hbar \omega\right)\left|M_{c v}(\vec{k})\right|^{2},
\end{aligned}
$$


where (Eq. (2.8))

$$
M_{c v}(\vec{k})=\sum_{n} \frac{\langle c, \vec{k}|\hat{\alpha} \cdot \vec{p}| n, \vec{k}\rangle\langle n, \vec{k}|\hat{\alpha} \cdot \vec{p}| v, \vec{k}\rangle}{E_{n}(\vec{k})-E_{v}(\vec{k})-\hbar \omega} .
$$

The factor of $10^{16}$ converts $\beta_{\mathrm{cv}}$ from cgs units into $\mathrm{cm} / \mathrm{GW}$.

Because of the symmetry of the zinc blende structure, the integral over the $\mathrm{BZ}$ in Eq. (3.19) may be replaced by sum of 24 integrals over $1 / 24$ of the $B Z$ each. If we consider an arbitrary $\vec{k}$ vector inside one of the subvolumes of the $\mathrm{BZ}$, the 24 symmetry operations of the zinc blende point group will map $\vec{k}$ into each one of the other subvolumes once and only once. The set of unique wave vectors obtained by applying all of these roto-inversion operators to the initial arbitrary wave vector $\vec{k}$ is called the "star" of $\vec{k} .^{23}$ If we temporarily denote the Cartesian components of $\vec{k}$ as $(x, y, z)$, then the star of $\vec{k}$ consists of all six permutations of the coordinates in each of the following four vectors: $(x, y, z),(x, \bar{y}, \bar{z}),(\bar{x}, y, \bar{z})$, and $(\bar{x}, \bar{y}, z)$. We denote the set of vectors that comprise the star of $\vec{k}$ by $\mathrm{K}^{\star}$.

Appendix $\mathrm{C}$ shows how the zinc blende $\mathrm{BZ}$ can be segmented by symmetry. If the arbitrary vector $\vec{k}$ happens to lie on a boundary of one of the 1/24-BZ segments, then its star contains fewer than 24 unique vectors. For example, if $\vec{k}$ points along the $x$-axis, then the star of $\vec{k}$ contains only six vectors: $( \pm k, 0,0),(0, \pm k, 0)$, and $(0,0, \pm k)$. Thus, boundary points are special. We make the assumption that the number of $\vec{k}$ vectors lying in special directions is neglible with respect to the total number of $\mathrm{k}$ vectors. This allows us to disregard boundary symmetries and to approximate a $1 / 24$ subvolume of the $\mathrm{BZ}$ as a sum of volumes of discrete cubes. If a cube extends over the edge of the subvolume, we multiply its volume by whatever fraction lies inside the $1 / 24 \mathrm{BZ}$ segment. All quantities in each cube are treated as if they are cinstant, and they are evaluated at the cube's center. The integral over a subvolume of the $\mathrm{BZ}$ is then the sum of the terms from each cube times the volume of the cube that lies inside the $\mathrm{BZ}$ subvolume. By modeling the $\mathrm{BZ}$ 
in this fashion, the round-off ersor in discretizing each $\mathrm{BZ}$ subvolume is about $3 \%$. This significantly reduces the error in the calculation and is responsible for the appearance of spectral features that are real but that have not previously been computed. (See the discussion of Figure 3-4.)

Because the Hamiltonian operator of a crystal is invariant under any symmetry operation of the crystal, the energy $E(\vec{k})$ is the same for all wave vectors in the star of $\vec{k}$. That is if $\vec{k} \in K^{\star}$ and if $\vec{k}^{\prime} \in K^{\star}$, then $E(\vec{k})=E\left(\vec{k}^{\prime}\right)$. Thus, we may rewrite the integral of Eq. (3.19) as follows:

$$
\begin{array}{r}
\int_{B Z} d^{3} \vec{k} \delta\left(E_{c v}(\vec{k})-2 \hbar \omega\right)\left|M_{c v}(\vec{k})\right|^{2}=\int_{\frac{1}{24} B Z} d^{3} \vec{k} \delta\left(E_{c v}(\vec{k})-2 \hbar \omega\right) \\
\times \sum_{\vec{k} \in K^{*}}\left|M_{c v}(\vec{k})\right|^{2} .
\end{array}
$$

Computing $\beta(\omega)$ now reduces to two problems: how to model a delta function and how to express the sumration in Eq. (3.21).

We address the issue of the delta function first. The delta function is modeled as a function whose value is either a constant or zero, depending on whether for a given $\vec{k}$, the value $\left|E_{c v}(\vec{k})-2 \hbar \omega\right|$ is less than or not less than some arbitrary tolerance $(\Delta \omega / 2)$. Specifically, one chooses an arbitrary value of $\Delta \omega$ and then

$$
\delta\left(E_{c v}(\vec{k})-2 \hbar \omega\right) d^{3} \vec{k} \approx \frac{\left(\Delta^{3} k\right)}{\hbar \Delta \omega} H\left(\frac{E_{c v}(\vec{k})-2 \hbar \omega}{\hbar \Delta \omega}\right)
$$

where $\Delta^{3} k$ is the volume in the $\mathrm{BZ}$ of the little discrete cube to which $\vec{k}$ points and where $\mathrm{H}$ was defined in Eq. (3.15).

The numerical pitfall in this techique is that the Brillouin zone must be subdivided sufficiently finely to avoid spikes and nulls due to sampling errors, but solving the pseudo wave equation at a large number of points is very time consuming. To circumvent this problem, we divided a $1 / 24$ subvolume of the $\mathrm{BZ}$ into a coarse grid of cubes and then subdivided each cube into a fine grid of tiny cubes. We computed the pseudo wave functions and band energies on the coarse grid. From these 
we computed the matrix elements $[\hat{\alpha} \cdot \vec{p}]_{i j}$ at each point in the coarse grid. Then we interpolated linearly over the fine grid within each coarse cube to estimate the matrix elemenis everywhere as a function of $\vec{k}$.

In this way, we effectively sampled the $\mathrm{BZ}$ at over 60,000 points. Despite such high resolution, spikes still occurred in numerically evaluating the integral. We performed a running average over frequency with a "window" that was several times as wide as $\Delta \omega$, the initially desired resolution in frequency space. This procedure yielded adequate results for the linear optical properties. However, we still had spiking for $2 \hbar \omega>6.6 \mathrm{eV}$ in computing $\beta$, as mentioned.

Now we consider the effective transition matrix element $M_{c v}(\vec{k})$. Similar to the notation of Eq. (2.73), let us denote the direction of the vector $\vec{A}$ by a unitary vector $\hat{\alpha}$, where

$$
\hat{\alpha}=c_{1} e^{-i \phi_{1}} \hat{x}+c_{2} e^{-i \phi_{2}} \hat{y}+c_{3} e^{-i \phi_{3}} \hat{z}
$$

Since $\hat{\alpha}$ is unitary, $\hat{\alpha}^{*} \cdot \hat{\alpha}=1$. The sum over $K^{\star}$ that appears in Eq. (3.21) may be expanded as follows:

$$
\begin{aligned}
\sum_{\vec{k} \in K^{*}}\left|M_{c v}(\vec{k})\right|^{2} & =\left(c_{1}^{4}+c_{2}^{4}+c_{3}^{4}\right) M_{111} \\
& +2\left\{\begin{array}{c}
c_{1}^{2} c_{2}^{2} \cos \left(2 \phi_{1}-2 \phi_{2}\right) \\
+c_{2}^{2} c_{3}^{2} \cos \left(2 \phi_{2}-2 \phi_{3}\right) \\
+c_{3}^{2} c_{1}^{2} \cos \left(2 \phi_{3}-2 \phi_{1}\right)
\end{array}\right\} M_{1221} \\
& +\left(c_{1}^{2} c_{2}^{2}+c_{2}^{2} c_{3}^{2}+c_{3}^{2} c_{1}^{2}\right) M_{1122}
\end{aligned}
$$

where

$$
\begin{aligned}
& M_{1111} \equiv 8\left(\left|S_{11}\right|^{2}+\left|S_{22}\right|^{2}+\left|S_{33}\right|^{2}\right) \\
& M_{1221} \equiv 8 R e\left(S_{11} S_{22}^{*}+S_{22} S_{33}^{*}+S_{33} S_{11}^{*}\right) \\
& M_{1122} \equiv 8\left(\left|S_{12}+S_{21}\right|^{2}+\left|S_{23}+S_{32}\right|^{2}+\left|S_{31}+S_{13}\right|^{2}\right)
\end{aligned}
$$

and where

$$
S_{i j} \equiv \sum_{n} \frac{\left(\hat{x}_{i} \cdot \vec{p}\right)_{c n}\left(\hat{x}_{j} \cdot \vec{p}\right)_{n v}}{E_{n}(\vec{k})-E_{v}(\vec{k})-\hbar \omega}
$$


In Eq. (3.26) $\hat{x}_{i}$ and $\hat{x}_{j}$ denote real-valued unit vectors along Cartesian crystallographic axes. Including a factor of 2 for spin, the computed result for $\beta$ is, therefore,

$$
\begin{aligned}
\beta_{c v}= & 4 \times 10^{16} \frac{\omega}{n^{2} c^{?}}\left(\frac{e}{m \omega}\right)^{4}\left(\frac{1}{\hbar \Delta \omega}\right) \\
& \times \sum_{(i j k) \in 1 / 24 B Z}\left(\Delta^{3} k\right)_{i j k} H\left(\frac{E_{c v}(i j k)-2 \hbar \omega}{\hbar \Delta \omega}\right)\left|M_{c v}(i j k)\right|^{2} .
\end{aligned}
$$

The notation (ijk) refers to a discretized grid point in the $1 / 24 \mathrm{BZ}$ domain of integration.

\subsubsection{Comparison of the Pseudopotential to Other Theoretical Methods.}

For light polarized along the (100) axis of a zinc blende crystal, Figure 3-7 compares the pseudopotential result for $\beta(\omega)$ to theories from Chapter 2. The general agreement between the results of this pseudopotential calculation and those of the $o^{\prime}$ methods is fair. Among the other methods, the tunneling prediction is consistently higher and parabolic-band predictions are lower than the pseudopotential result. The lowest curve of all is our parabolic-band theory given by Eq. (2.41). Weiler's parabolic-band model is slightly higher than ours, but it is still generally less than the pseudopotential result. As mentioned in Chapter 2, Weiler's parabolic-band model is technically inappropriate for $\mathrm{ZnS}$ because it assumes that the spin-orbit splitting is large compared to the band-gap energy and that no transitions occur between the split-off band and the conduction band. However, it does admit intervalence transitions in the intermediate state which our simple parabolicband model does not.

For some polarizations then, previous theories are within $\pm 50 \%$ of the pseudopotential results. The chief failures of the simple models are that they do not account for the polarization- or frequency-dependence of $\beta(\omega)$, and they cannot predict detailed spectral shape. 


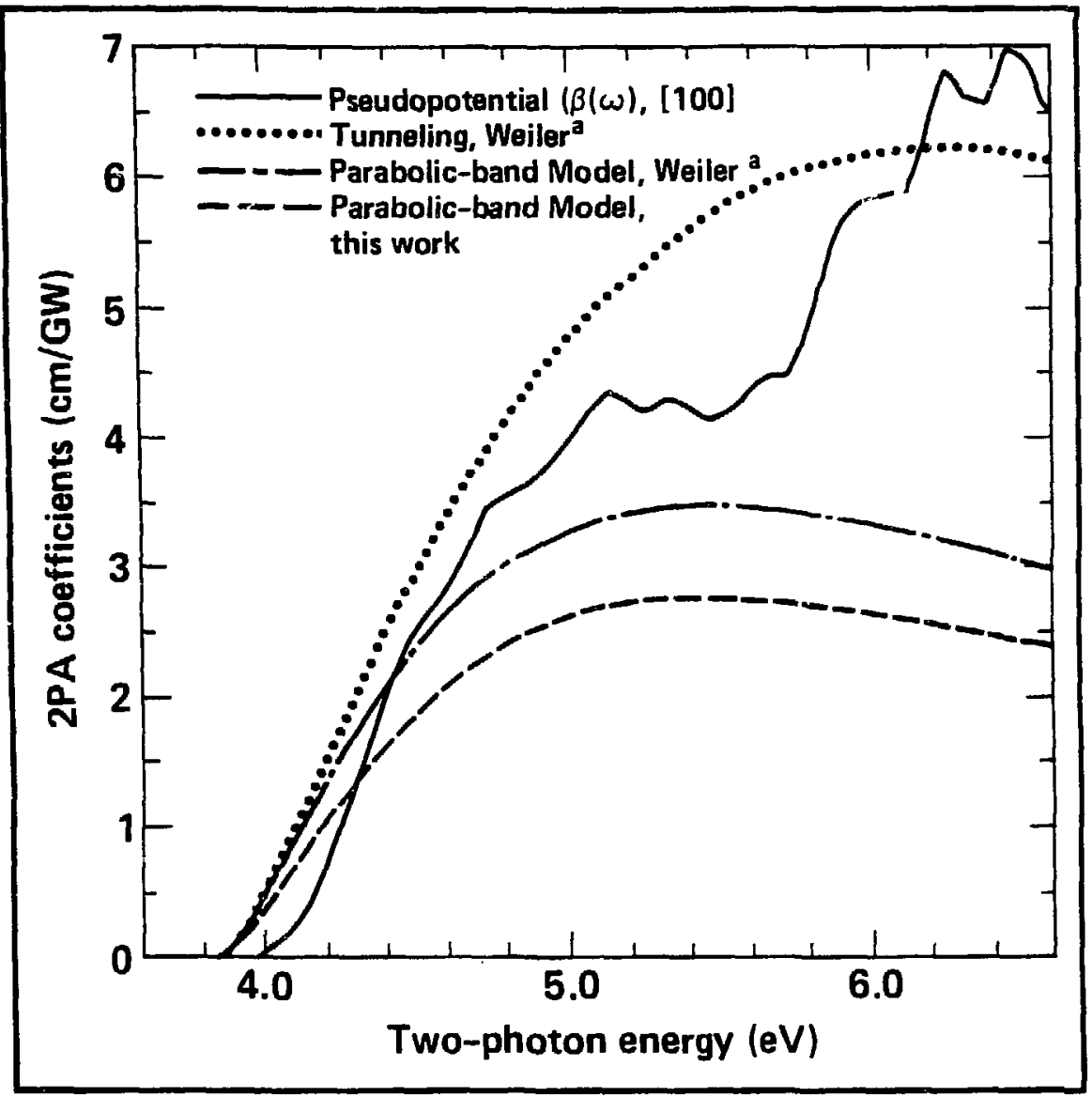

Figure 3-7. Comparison of Other Theories to the Pseudopotential Results.

"Ref. 24. 


\subsection{Summary.}

This chapter has discussed the calculation of $\beta$ by using pseudopotentials. Figure 3-6 showed that excellent agreement between theory and measurement can be obtained. This is the first time anyone has reported a two-photon absorption spectrum with a pseudopotential technique. The details of the calculation and the calibration of the relative data of Bae, Song, and Kim were discussed. Because of the consistency among the data of Bae, Song, and Kim, of Park and Waff, and of Panizza, the relative data of Bae et al. are believed to be calibrated now. This is corroborated by the pseudopotential calculation, and so we shall use $\mathrm{ZnS}$ as a measurement standard for the thermal lensing measurements that are to come in Chapter 5. 


\section{References}

1. M. L. Cohen and V. Heine, "The fitting of pseudopotentials to experimental data and their subsequent application," in Solid State Physics, 24, H. Ehrenreich, F. Seitz, and D. Turnbull, editors (Academic Press, New York, 1970).

2. N. W. Ashcroft and N. D. Mermin, Solid State Physics (Saunders College, Philadelphia, 1976).

3. M. L. Cohen, "The pseudopotential panacea," Physics Today 32(7), 40-47 (July 1979).

4. A. Vaidyanathan, A. H. Guenther, and S. S. Mitra, "Band-structure calculations of the two-photon absorption coefficients of GaAs, InP, CdTe, and ZnSe," Phys. Rev. B 24(4), 2259-62 (15 Aug. 1981).

5. Vaidya Nathan, A. H. Guenther, and S. S. Mitra, "Review of multiphoton absorption in crystalline solids," J. Opt. Soc. Am. B 2(2), 294-316 (Feb. 1985).

6. M. L. Cohen and T. K. Bergstresser, "Band structures and pseudopotential form factors for fourteen semiconductors of the diamond and zinc-blende structures," Phys. Rev. 141(2), 789-96 (Jan. 1966).

7. C. Y. Fong and Y. R. Shen, "Theoretical studies on the dispersion of the nonlinear optical susceptibilities in GaAs, InAs, and InSb," Phys. Rev. B 12(6), 2325-35 (15 Sept. 1975).

8. A. A. Martynov and A. E. Rozenson, "Quantum mechanical calculation of linear and nonlinear susceptibilities of an $\mathrm{HgGa}_{2} \mathrm{~S}_{4}$ crystal in the transparency range," Sov. Phys. Semicond. 14(10), 1137-39 (Oct. 1980) [Fiz. Tekh. Poluprovdn.(USSR) 14, 1908-12 (Oct. 1980)].

9. L. M. Falicov and P. J. Lin, "Band structure and Fermi surface of antimony," Phys. Rev. 141(2), 562-67 (Jan. 1966); P. J. Lin and L. M. Falicov, "Fermi surface of arsenic," Phys. Rev.142(2), 441-44 (Feb. 1966). 
10. T. K. Bergstresser and M. L. Cohen, "Electronic structure and optical properties of hexagonal CdSe, CdS, and ZnS," Phys. Rev 164(3), 1069-80 (15 Dec. 1967).

11. J. R. Chelikowsky and M. L. Cohen, "Nonlocal pseudopotential calculations for the electronic structure of eleven diamond and zinc-blende semiconductors," Phys. Rev. B 14(2), 556-82 (15 July 1976).

12. M. Bennett and J. C. Inkson, "Self-energy effects in zinc sulphide," J. Phys. C: Solid State Phys. (Great Britain) 12(2), 283-92 (28 Jan. 1979).

13. C. S. Wang and B. M. Klein, "First-principles electronic structure of $\mathrm{Si}$, Ge, GaP, GaAs, ZnS, and ZnSe, ${ }^{n}$ Phys. Rev. B 24(6), 3393-429 (15 Sept. 1981).

14. D. Brust, "Electronic spectra of crystalline germanium and silicon," Phys. Rev. 134(5A), A1337-53 (1 June 1964).

15. F. Wooten, Optical Properties of Solids (Academic Press, New York, 1972).

16. M. Cardona and G. Harbeke, "Optical properties of w'urtzite-type crystals in the fundamental absorption region," in Proc. Intern. Conf. Semicond., Paper O2-4, 217-23 (Dunod Cie, Paris, 1964).

17. J.W. Baars, "The fundamental reflectivity of $\mathrm{ZnS}$ single crystals with $3 \mathrm{C}, 2 \mathrm{H}$, $4 \mathrm{H}, 6 \mathrm{H}$, and 10H structure," II-VI Semiconducting Compounds - 1967 Intern. Conf., D. G. Thomas, editor, 631-68 (Benjamin, New York, 1967).

18. Eric W. Van Stryland, H. Vanherzeele, M. A. Woodall, M. J. Soileau, A. L. Smirl, S. Guha, and T. F. Boggess, "Two-photon absorption, nonlinear refraction, and optical limiting in semiconductors," Opt. Engineering 24(4), 613-23 (July/Aug. 1985).

19. Y. Bae, J. J. Song, and Y. B. Kim, "Photoacoustic study of two-photon absorption in hexagonal ZnS," J. Appl. Phys. 53(1), 615-19 (Jan. 1982).

20. M. L. Cohen, "Pseudopotentiâl calculations of II-VI compounds," in II-VI Semiconducting Compounds, 1967 International Conference, D. G. Thomas, editor, 
462-502 (W. A. Benjamin, Inc., NY, 1967). (See p. 480.)

21. M. D. Galanin and Z. A. Chizhikova, "Angular dependence to two-photon absorption in a zinc-sulfide crystal," Zh ETF Pis. Red. 18(10) 348-51, 571-74 (20 Nov. 1968).

22. E. Panizza, "Two-photon absorption in ZnS," Appl. Phys. Lett. 10(10), 256-66 (15 May 1966).

23. TinkhamGroup Theory and Quantum Mechanics (McGraw-Hill, N. Y., 1964).

24. M. H. Weiler, "Nonparabolicity and exciton effects in two-photon absorption in zincblende semiconductors," Solid State Commun. (Great Britain) 39(8), 937-40 (Pergamon Press, Aug. 1981). 


\section{Chapter 4}

\section{DUAL-BEAM PHOTOTHERMAL LENSING}

\subsection{Introduction.}

This chapter provides a detailed background for the optical absorption measurements in Chapter 5. Section 4.0 reviews past work in thermal lensing. Section 4.1 gives a simplified picture of thermal lensing, from which we estimate the size of the signal one might expect. Section 4.2 treats thermal lensing with two Gaussian beams, one of which (the pump) is imagined infinitely wider than the other (the probe). That section is drawn primarily from work by Twarowski and Kliger. ${ }^{1}$ Section 4.3 considers the effect of having a pump beam whose diameter is not much greater than that of the probe. Spherical aberration must then be accounted for. Section 4.3 contains the key result of this chapter, Eq. (4.53).

Photothermal lensing is a technique for measuring weak optical absorption coefficients by detecting small, optically induced temperature changes in a sample. In liquids, linear absorption as weak as one part in $10^{-5}$ has been measured. Some workers have stated that this sensitivity can be improved by at least an order of magnitude, perhaps two. ${ }^{2}$ The demonstrated capability to measure two-photon absorption in highly transparent liquids ${ }^{1}$ makes thermal lensing of interest for measuring absolute two-photon absorption in highly transparent solids, also. ${ }^{3}$ Henesian and Weber ${ }^{4}$ used thermal lensing to detect two-photon-induced color centers in a borosilicate glass, but they did not determine the absolute two-photon absorption coefficients of their sample.

Figure 4-1 illustrates dual beam photothermal lensing. Optical absorption of a laser "pump beam" changes the local temperature distribution inside a sample. As a result, the refractive index $n$ of the sample, through its temperature dependence, 


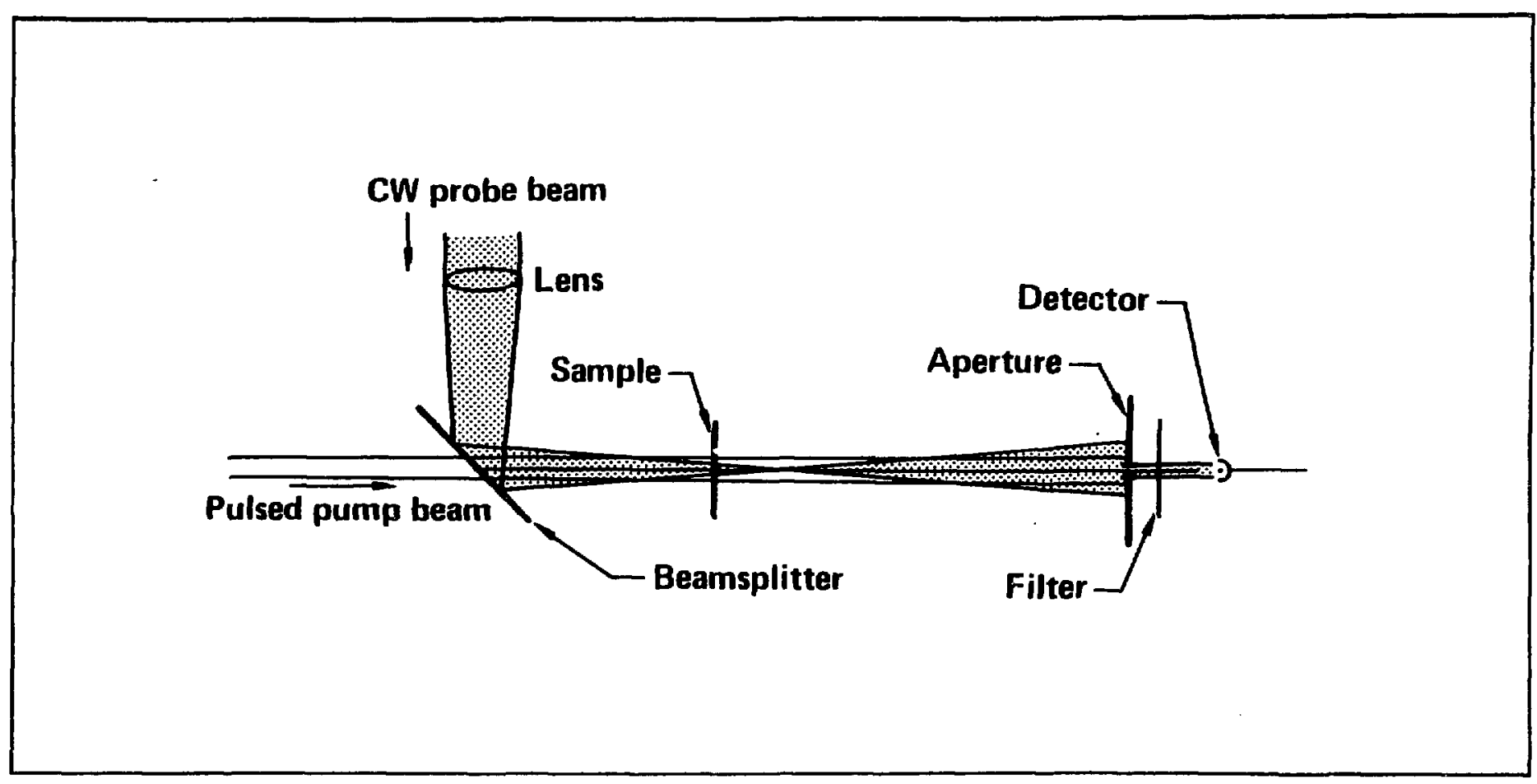

Figure 4-1. Dual Deam Thermal Lensing. The nommiform healing in the sample caused by absorption of a spatially varying pump beam induces a thermal gradient index lens, which changes the far-field axial intensity of a weak probe beam. 
exhibits a spatial variation, forming a thermal lens. The pump and probe beams may be the same beam, although this is not necessary. ${ }^{5}$ When the probe beam is centered on the thermal lens, the principal effect of the lens is to change the divergence of the probe. The fractional change in the far-field intensity is then measured. Related techniques include photothermal deflection, ${ }^{2}$ photothermal interferometry, ${ }^{6}$ photoacoustic detection, ${ }^{7}$ photocalorimetry, ${ }^{8}$ photothermal displacement, ${ }^{9}$ and the mirage effect ${ }^{10}$ ("transverse photothermal deflection" ${ }^{2}$ ).

Thermal lensing was discovered during intracavity laser spectroscopy experiments and was first reported by Gordon et al. in $1964 .{ }^{11}$ It was subsequently improved by $\mathrm{Hu}$ and Whinnery, who moved the sample outside the cavity for better laser stability. ${ }^{12} \mathrm{Hu}$ and Whinnery first suggested the idea of using thermal lensing techniques to measure nonlinear optical properties, although they themselves did not do so. Long, Swofford, and Albrecht introduced dual beam thermal lensing spectroscopy, ${ }^{5}$ and Twarowski and Kliger first applied a dual beam technique to nonlinear absorption spectroscopy. ${ }^{1}$ In a clever hybridization of single-beam and dual-beam techniques, Fang, Gustafson, and Swofford used as a pump beam a dye laser that had been synchronously pumped by a mode-locked argon laser. ${ }^{13}$ The quasi-continuous nature of the pump ieam enabled them to use lock-in techniques for a high signal-to-noise ratio, and the picosecond pulses of the synchronously pumped dye laser enabled them to measure nonlinear absorption in organic liquids.

Photothermal techniques are suited for measuring a variety of optical, thermal, and chemical properties: nonlinear absorption, ${ }^{1}$ linear absorption, ${ }^{2}$ fluorescent yield, ${ }^{14}$ Mössbauer spectroscopy, ${ }^{15}$ specific heat, ${ }^{16}$ thermal diffusivity, ${ }^{17} \frac{\partial n}{\partial T},{ }^{18}$ rates of reaction, ${ }^{19}$ and photochemical kinetics, ${ }^{20}$ to name several. Measurements in very small samples (of the order of ten optical wavelengths in diameter) are also a potentially very interesting area of research. ${ }^{21}$ Some unusual measurements that 
have been made photothermally include measuring the specific heat of chablis at $40 \mathrm{~F}^{22}$ the reflectance of a single human hair, ${ }^{23}$ and the absorption coefficient of aspirin. ${ }^{24}$ Appendix F contains a bibliography on thermal lensing.

Compared to other photothermal techniques, photothermal lensing has a unique combination of advantages for measuring weak optical absorption in solids. Besides its sensitivity, which is comparable to that of thermal deflection, it probes the volume of a sample. This capability distinguishes it from surface techniques like photothermal displacement and the mirage effect. It is also a non-contact measurement. This eliminates the false-signal problems associated with scattered light in, for example, photoacoustics and photocalorimetry.

Applying thermal lensing to solids has certain handicaps, some of which are due to the nature of solids. For example, the partial derivative with respect to temperature $\partial n / \partial T$ is typically ten times smaller in solids than in liquids. ${ }^{11,25}$ Therefore, thermal lensing is generally less sensitive in solids than in liquids. Additionally, thermal lensing (like other photothermal methods) depends upon a knowledge of several thermal parameters, some of which may not be precisely known. These include the efficiency with which absorbed photons are converted into local heat and the effective value of the thermo-optic coefficient.

\subsection{A Geometrical Optics Model of Pulsed Thermal Lensing.}

Consider a collimated, radially symmetric pump beam of intensity $I(r)$ and pulse length $\tau$. The pulse length is assumed to be short relative to thermal decay times. Near the center of the beam, $I(\tau)$ has the following form:

$$
I(r)=I_{0}-I_{0} r^{2} / 2 w_{p}^{2}
$$

where $I_{0}$ is the axial $(r=0)$ intensity and $w_{p}$ is an effective beam width. Let the beam be absorbed by a thin slab of material whose optical absorption coefficient is $\alpha_{\text {opt }}$. Absorption of the pump beam leads to a local change of temperature $\Delta T$ 
that also is radially symmetric. Since the pulse duration $\tau$ is short, it is convenient to work in terms of fluence $\Gamma$, where

$$
\Gamma(r)=I(r) \tau
$$

We assume that spatial variations in $\Gamma$ are small relative to an optical wavelength. The local heat per unit volume due to absorption is $Q=(1-\eta) \alpha_{\text {opt }} \Gamma$, where $1-\eta$ is the amount of heat released per energy absorbed. The local temperature change due to optical absorption of the incident fluence $\Gamma$ is therefore

$$
\triangle T=(1-\eta) \alpha_{o p t} \Gamma / C
$$

where $C$ is the specific heat per unit volume. For a typical glass, $C$ is about $2.0 \mathrm{~J}$ $\mathrm{cm}^{-3} / \mathrm{K},{ }^{26}$ and for now we suppose $1-\eta$ to be unity. If $\alpha_{o p t}$ is weak, the change in temperature is small. Taking $\alpha_{o p t}=10^{-3} \mathrm{~cm}^{-1}$ and $\Gamma=10 \mathrm{~J} / \mathrm{cm}^{2}$ gives $\Delta T<0.005 \mathrm{~K}$. The thermal change in the refractive index, $\Delta n$, is therefore small and is given by

$$
\triangle n=\frac{\partial n}{\partial T} \triangle T
$$

According to Eqs. (4.1)-(4.3), $\Delta n$ has a non-uniform spatial profile, and so the sample becomes a "thermal lens." The focusing power of the lens is $\triangle(1 / f)$, where

$$
\triangle 1 / f=-\ell\left[\frac{\partial^{2} \triangle n}{\partial r^{2}}\right]_{r=0} .
$$

In Eq. (4.4), $\ell$ is the sample thickness. We have temporarily neglected any thermal changes in $\ell$ for the sake of clarity. It follows that

$$
\Delta 1 / f=\left[\alpha_{o p t} \ell / C w_{p}^{2}\right] \frac{\partial n}{\partial T} \Gamma_{0},
$$

where the subscript 0 on $\Gamma$ denotes the axial fluence. For a $1.0-\mathrm{cm}$ thick sample, a 0.02-cm diameter pump beam, a typical $\partial n / \partial T$ of $10^{-5} \mathrm{~K}^{-1}$ in glass, ${ }^{27}$ and $\Gamma_{0}, \alpha_{\text {opt }}$, and $C$ as before, $\triangle(1 / f)$ is about 0.05 diopters, corresponding to a focal length of 20 meters. 
Equation (4.6) shows that the focusing power of the thermal lens increases with pump beam fluence and sample thickness and decreases as the reciprocal of the specific heat. The sign of $\Delta(1 / f)$ is positive for a focusing lens and negative for a diverging lens. It is determined by the sign of $\partial n / \partial T$.

Let us now see what effect $\Delta(1 / f)$ has upon the probe beam. In a geometrical optics picture, the probe beam is a set of rays emanating from a point a distance $R$ in front of the sample as in Figure 4-2. If the axial intensity of the probe beam is denoted by $I_{1}$ at the sample and by $I_{2}$ a distance $z$ beyond the sample, then from geometrical optics, one has

$$
I_{2}=I_{1} /(1+z / R)^{2}
$$

Superimposing a thin lens upon the sample alters the initial divergence of the probe beam, and the intensity $I_{2}$ becomes $I_{2}^{\prime}$, where

$$
I_{2}^{\prime}=I_{1} /(1+z / R-z \triangle 1 / f)^{2} .
$$

The relative change in $I_{2}$ is defined as $\triangle I_{2} / I_{2}^{\prime}$, with $\triangle I_{2}=I_{2}^{\prime}-I_{2}$. For a weak lens $(R \triangle(1 / f) \ll 1)$, the relative change in $I_{2}$ is, to lowest order in $\triangle(1 / f)$,

$$
\triangle I_{2} / I_{2}^{\prime}=2 R \triangle(1 / f) /(1+R / z)
$$

Using $\Delta(1 / f)=0.05 \mathrm{~m}^{-1}, z=2 \mathrm{~m}$ and $R=10 \mathrm{~cm}$, one has $\Delta I_{2} / I_{2} \sim 0.01$. This order of magnitude characterizes many thermal lensing measurements.

Equation (4.9) demonstrates several useful points. First, the relative change of intensity is a product of two terms, one of which (viz. $\Delta(1 / f)$ ) depends on the sample properties and the other of which depends on the probe beam configuration. Second, to first order, $\Delta(1 / f)$ has no effect upon the probe beam if the beam is focused at the sample $(R=0)$. This is a general consequence of the thin lens approximation. Third, the observed change of intensity may be either positive 


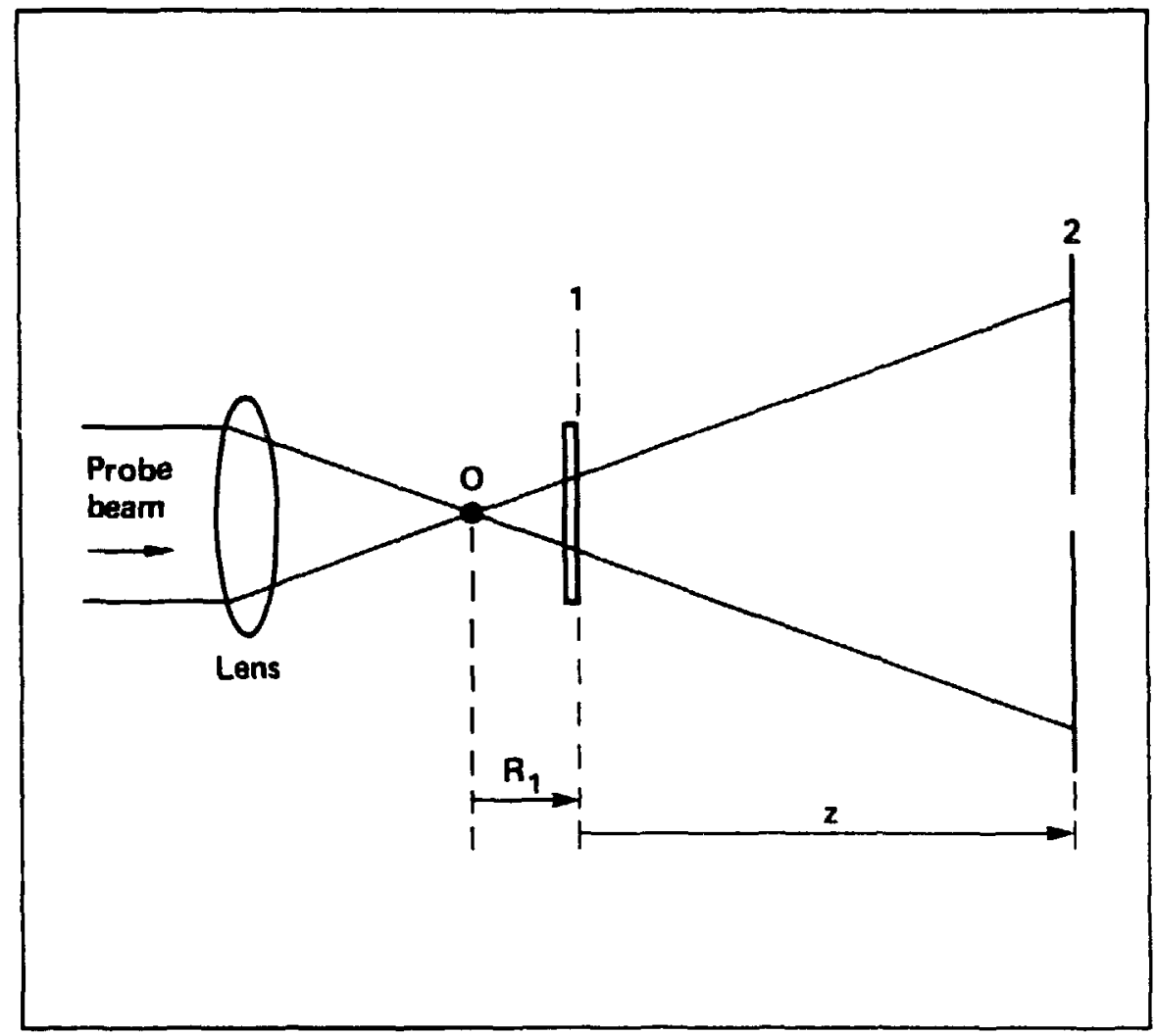

Figure 4-2. Key Parameters of the Probe Beam. O, center of wavefront; $R_{1}$, radius of curvature; $z$, distance from sample plane 1 to reference plane 2. 
(an increase of intensity) or negative (a decrease), depending upon the sign of $(\Delta 1 / f) /(1+z / R)$. For positive $\Delta(1 / f), \Delta I_{2} / I_{2}$ is positive if and only if the probe beam is not initially focused between the sample and the plane of observation (i.e., if $1+z(R>0)$. Figure 4-3 illustrates two probe beam configurations, showing qualitatively the thermal lensing signals due to a short probe pulse. The polarity of the thermal lensing signal is useful for verifying the sign of $\partial n / \partial T$ in certain samples. Equation (4.9) may be rewritten as follows:

$$
\triangle I_{2}^{\prime} / I_{2}^{\prime}=\frac{\alpha_{o p t} \Gamma_{0} \ell}{w_{p}^{2} C} \frac{\partial n}{\partial T} .
$$

This shows explicitly that the relative change of intensity in the plane of observation is proportional to the optical absorption coefficient, to lowest order.

\subsection{Thermal Lensing of Two Gaussian Beams.}

This section refines the preceeding geometrical optics model of thermal lensing by considering pump and probe beams that are Gaussian functions of the transverse coordinates and by accounting for thermal diffusion. The method of this section is adapted from that of Twarowski and Kliger. ${ }^{1}$ The thermal diffusion equation gives an expression for the optically induced temperature change, $\Delta T$, from which we find the focusing power of the thermal lens, $\Delta(1 / f)$. The effect of $\Delta(1 / f)$ on the far-field axial intensity of the $\mathrm{cw}$ Gaussian probe beam is then determined. The width of the probe beam at the sample is assumed much less than the width of the thermal lens. Both linear and nonlinear absorption are considered. As before, the purnp beam pulse length is assumed much shorter than thermal diffusion times. The sample is treated as an infinitely wide, thin slab, and surface boundary effects and radiative transport of energy are assumed to be unimportant compared to thermal conduction inside the slab. (Appendix $\mathbf{E}$ discusses this assumption.) Thermal diffusion along the axis of the thermal lens is also disregarded, since the optical absorption depth is long compared to the thickness of the samples of interest $\left(\alpha_{\text {opt }} \ell \ll 1\right)$. 


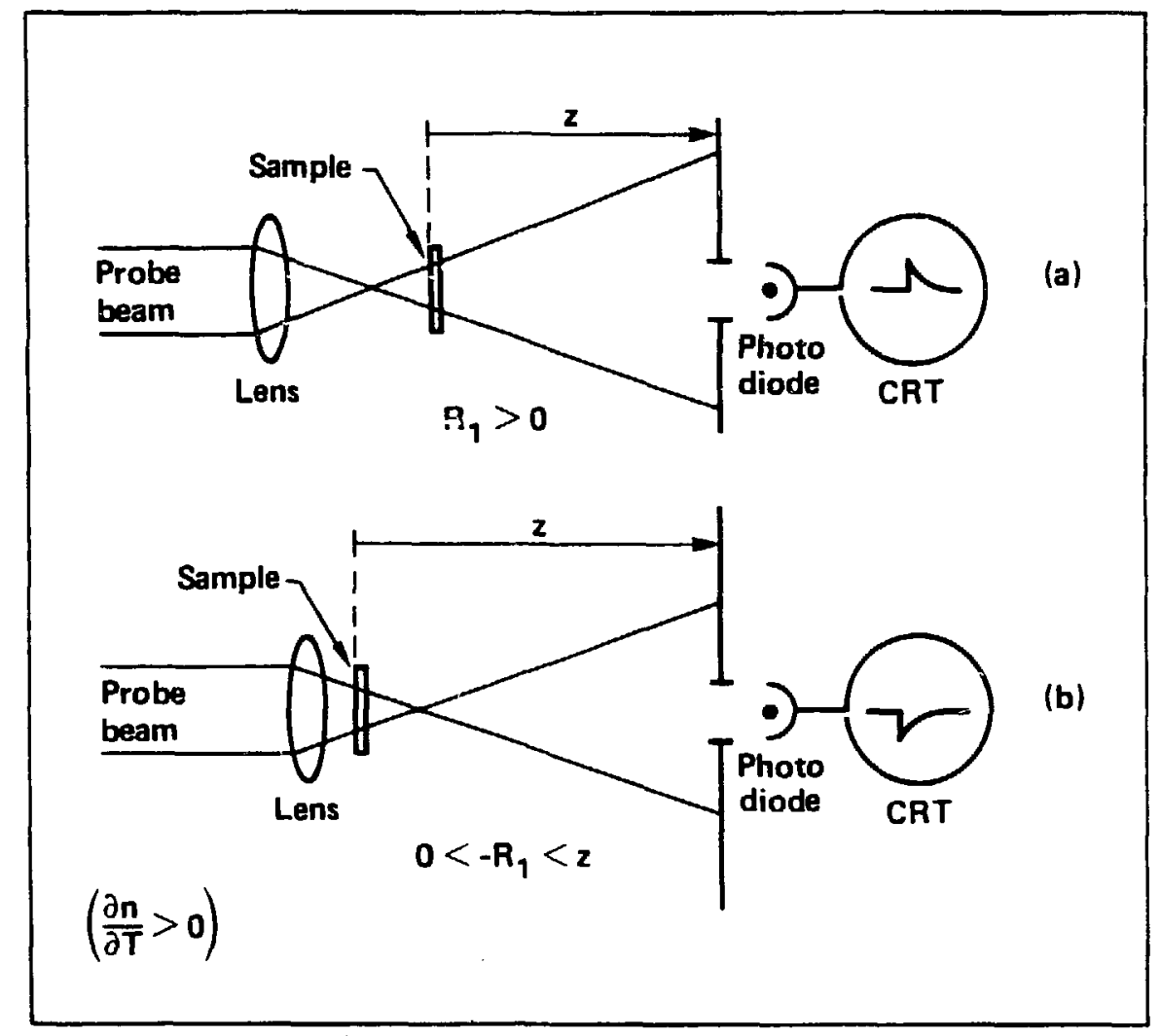

Figure 4-3. Effect of Sample Position upon Thermal Lensing Signal. (a) The sample is between the focus of the probe and the plane of observation. The thermal lens increases the distant axial intensity of the probe beam. (b) The focus of the probe beam is between the sample and the plane of observation. The thermal lens diminishes the distant axial intensity of the probe beam. A positive $\partial n / \partial T$ has been assumed, which is typical of many highly transparent glasses. 
The thermal diffusion equation describes the flow of heat in a continuous body. For an isotropic or cubic material that has no internal heat sources, the equation is

$$
C \partial T / \partial t=\vec{\nabla} \cdot\left(k_{t h} \vec{\nabla} T\right)
$$

where $k_{t h}$ is the thermal conductivity. Implicit in Eq. (4.11) is the assumption that every point in the body is characterized by a well defined local temperature. In an insulating solid the validity of the local-temperature approximation is equivalent to the criterion that the mean free path of phonons be much less than any macroscopic dimension of interest, such as an optical wavelength. At room temperature this criterion is easily met in solids. For example, the mean free path of a phonon in crystalline quartz is about $40 \AA$ at $0 \mathrm{C}, 100$ times smaller than a typical optical wavelength. ${ }^{27}$ In amorphous insulators, the mean free paths are even lower, due to the lack of structural periodicity. The thermal diffusion equation should therefore apply to all thermal lensing cases of interest in this study.

Neglecting the dependence of $k_{t h}$ and $C$ upon temperature and neglecting the variation of $T$ along the axis of the thermal lens simplifies the diffusion equation to

$$
\partial T / \partial t=D\left(\partial^{2} T / \partial r^{2}+r^{-1} \partial T / \partial r\right)
$$

where $r$ is a radial coordinate transverse to the axis of the thermal lens and $D$ is thermal diffusivity,

$$
D=k_{t h} / C
$$

To neglect axial variations in $T$ is consistent with the assumption of weak absorption. Azimuthal variations around the axis of the thermal lens have been neglected in Eq. (4.12) since the source of heat (the pump laser beam) is circularly symmetric.

From Eq. (4.12) one can easily estimate the duration of the thermal lens by assuming a Gaussian temperature profile of the form $T(t) \exp \left(-2 \tau^{2} / w_{p}^{2}\right)$. At $r=0$ 
the temperature $T(t)$ decays exponentially with a time constant equal to $w_{p}^{2} / 8 D$. For an optical glass, $k_{t h}$ is about $0.01 \mathrm{~W} / \mathrm{cm}$ and $C$ is about $2 \mathrm{~J}-\mathrm{cm}^{-3} / \mathrm{K}$. Typical values of the Gaussian pump beam width in thermal lensing measurements are of the order of $z=0.01 \mathrm{~cm}$. This gives $w_{p}^{2} / 8 D=2.5 \times 10^{-3}$ seconds.

If the pump beam pulse and the nonradiative electronic relaxation are fast relative to thermal diffusion, then the initial heat is deposited quasi-instantaneously into the sample. (The conditions for instantaneous heat deposition are easily met for nanosecond pulses in undoped room-temperature insulators, where electron-hole recombination times are sub-microsecond. ${ }^{28}$ ) Letting $Q(r)$ denote the heat density in the sample immediately after the pump beam has been absorbed, the initial condition on $T(r, t)$ is

$$
T(r, 0)=Q(r) / C
$$

The function $Q(r)$ is defined as

$$
Q(r)=\int_{\tau} d t \alpha_{o p t} I(r, t)
$$

Denoting the m-photon absorption coefficient by $\beta^{(m)}$, the effective optical absorption coefficient $\alpha_{\text {opt }}$ is

$$
\alpha_{o p t}=\beta^{(1)}+\beta^{(2)} I+\cdots+\beta^{(m)} I^{m}+\cdots
$$

(In the notation from Chapter $2, \beta^{(1)}=\alpha$ and $\beta^{(2)^{\prime}}=\beta$.)

The boundary condition on $T$ in the infinitely wide sample is

$$
\lim _{r \rightarrow \infty} T(r, t)=T_{0}
$$

The constant temperature $T_{0}$ is the temperature of the sample prior to the arrival of the pump beain. Since thermal conduction is assumed to dominate the heat transport, loss of heat across the surface of the sample has been neglected. 
It is convenient to represent the change of temperature as a superposition of term॰ $T^{(m)}$, where $T^{(m)}$ is the temperature change due to m-photon absorption:

$$
\begin{aligned}
\Delta T(r, t) & =T(r, t)-T_{0} \\
& =T^{(1)}+T^{(2)}+\cdots+T^{(m)}+\cdots,
\end{aligned}
$$

Each $T^{(m)}$ satisfies the thermal diffusion equation separately, subject to its own boundary values and initial conditions:

$$
\lim _{r \rightarrow \infty} T^{(m)}(r, t)=0, \quad m=1,2, \ldots
$$

and

$$
T^{(m)}(r, 0)=Q^{(m)}(r) / C, \quad m=1,2, \ldots
$$

where

$$
Q^{(m)}(r)=\int_{\tau} \beta^{(m)} I^{m} d t
$$

The decomposition of $T$ in Eq. (4.18) is justified, because the diffusion equation is linear when each $Q^{(m)}$ is independent of $T$, which is the case for small changes of temperature.

In order to determine $Q^{(m)}(r)$, the intensity $I$ must be known. For a pump beam with a Gaussian spatial profile the intensity is

$$
I(r, t)=I_{0}(t) \exp \left(\frac{-2 r^{2}}{w_{p}^{2}}\right),
$$

where $I_{0}(t)$ is the time-dependent axial intensity and $w_{p}$ is the Gaussian beam width. We make no assumptions about the temporal pulse shape. Instead, we introduce the pulse shape factor $h^{(m)}$, defined as ${ }^{1}$

$$
h^{(m)}=\int_{\tau}\left(\frac{I_{0}(t)}{\Gamma_{0}}\right)^{m} d t
$$

where

$$
\Gamma_{0}=\int_{\tau} I_{0}(t) d t
$$


is the axial fluence of the pump beam. The dimensions of $h^{(m)}$ are $s^{-m+1}$. Note that $h^{(1)}=1$. Combining Eqs. (4.21)-(4.24) gives

$$
Q^{(m)}(r)=\beta^{(m)} \exp \left(\frac{-2 m r^{2}}{w_{p}^{2}}\right) \Gamma_{0}^{m} h^{(m)}
$$

Using Eq. (4.3), we now determine $T^{(m)}$ for arbitrary m-photon absorption. We use the method of equivalent sources, as discussed in standard texts on heat equation. ${ }^{29}$ Let $G\left(r, r^{\prime}, t\right) r^{\prime} d r^{\prime} d \theta^{\prime} / C$ denote the temperature at a position $r$ and time $t$, given an instantaneous deposition of heat at position $r^{\prime}$ and time 0 from a cylindrical source whose cross sectional area is $r^{\prime} d r^{\prime} d \theta^{\prime}$ and whose total heat per unit volume is unity at every point along the source. Then $G\left(r, r^{\prime}, t\right) Q^{(m)}\left(r^{\prime}\right) r^{\prime} d r^{\prime} d \theta^{\prime} / C$ is the temperature for a line source at $r^{\prime}$ whose heat density is $Q^{(m)}\left(r^{\prime}\right)$. Summing over all sources gives the temperature change at $(\tau, t)$ as

$$
T^{(m)}(r, t)=2 \pi \int_{0}^{\infty} r^{\prime} d r^{\prime} G\left(r, r^{\prime}, t\right) Q^{(m)}\left(r^{\prime}\right) / C,
$$

where, for circularly symmetric sources, ${ }^{1,11,29}$

$$
G\left(r, r^{\prime}, t\right)=(4 \pi D t)^{-1} \exp \left[\frac{-\left(r^{2}+r^{\prime 2}\right)}{4 D t}\right] \times I_{0}\left(\frac{r r^{\prime}}{2 D t}\right)
$$

In Eq. (4.27), $I_{0}$ denotes a modified Bessel function of the first kind, not intensity. Integrating Eq. (4.26) yields

$$
T^{(m)}(r, t)=\frac{(1-\eta) \beta^{(m)} h^{(m)} \Gamma_{0}^{m}}{C} \frac{\exp \left[-\left(2 m r^{2} / w_{p}^{2}\right) /\left(1+8 m D t / w_{p}^{2}\right)\right]}{1+8 m D t / w_{p}^{2}} .
$$

The total laser-induced temperature change as a function of position and time, $\triangle T(r, t)$, is the sum of all terms $T^{(m)}$ given by Eq. (4.28).

Having found $\Delta T$, we now determine $\Delta(1 / f)$. In Section 4.1 , thermal change in length was neglected; however, it should not be ignored in solids. For example, given a 1-cm thick sample and an acoustic velocity on the order of $5 \times 10^{5} \mathrm{~cm} / \mathrm{s}$ (as is typical of crystals and glasses) ${ }^{27}$ there is ample time for thermal expansion to 
occur during thermal diffusion. The expansion is constrained, though. Longitudinal expansion is constrained, because the heated cylinder of sample, the thermal lens, must overcome the viscous shear forces of the surrounding unheated sample in order to stretch. Radial expansion is contrained because the bulk sample resists the outward pressure of the small heated volume. This constrained expansion is complicated and leads to a set of conditions for which $\partial(n \ell) / \partial T$ may not be readily available from tables of thermal parameters. Therefore, it is to be understood below that the partial derivatives with respect to temperature are effective values, not necessarily free-expansion values. (Note that liquids generally do not have this problem, due their small viscosities.)

The thermal change in optical path has two contributions, the change due to the sample, $\Delta(n \ell)$, plus the change due to displacement of air by the expanding sample, $-\Delta \ell$. Foc using power is the curvature of the total change in optical path length,

$$
\triangle(1 / f)=\frac{-\partial(n \ell-\ell)}{\partial T}\left[\frac{\partial^{2} \Delta T}{\partial r^{2}}\right]_{r=0} .
$$

As in Eq. (4.18), we expand Eq. (4.29) as a sum of m-photon contributions,

$$
\Delta(1 / f)=\Delta(1 / f)^{(1)}+\Delta(1 / f)^{(2)}+\cdots,
$$

where

$$
\Delta(1 / f)^{(m)}=\frac{-\partial(n \ell-\ell)}{\partial T}\left[\frac{\partial^{2} T^{(m)}}{\partial r^{2}}\right]_{r=0} .
$$

Equations (4.29) and (4.31) assume that the effect of the thermal lens is important only near the optical axis, in accordance with the assumption that the pump beam is much wider than the probe beam at the sample.

We now consider the effect of a thin, parabolic thermal lens on a cw probe beam that is spatially Gaussian. As in Section 4.1, the lens changes the divergence and hence the far-field intensity of the probe beam. Let $1 / R_{1}$ denote the on-axis phase 
curvature of the probe beam at the sample in the absence of a thermal lens. The thermal lens changes that curvature to $1 / R_{1}^{\prime}$, where

$$
1 / R_{1}^{\prime}=1 / R_{1}-\triangle(1 / f)
$$

As before, a positive curvature implies a diverging wave, and a positive $\Delta(1 / f) \mathrm{im}$ plies a focusing lens. Let $I_{1}$ continue to denote the axial intensity of the probe beam at the sample, and let $I_{2}^{\prime}$ and $I_{2}$ denote the intensities a distance $z$ beyond the sample, with and without a thermal lens, respectively. Let $w_{1}, w_{2}^{\prime}$, and $w_{2}$ denote the corresponding beam widths. From the laws of Gaussian optics, we have ${ }^{30}$

$$
\begin{aligned}
& I_{2}=I_{1} \frac{w_{1}^{2}}{w_{2}^{2}} \\
& I_{2}^{\prime}=I_{1} \frac{w_{1}^{2}}{w_{2}^{\prime 2}}
\end{aligned}
$$

and

$$
\begin{aligned}
w_{2}^{2} & =w_{1}^{2}\left(1+\frac{z}{R_{1}}\right)^{2}+\left(\frac{\lambda z}{\pi w_{1}}\right)^{2} \\
w_{2}^{\prime 2} & =w_{1}^{2}\left(1+\frac{z}{R_{1}}-z \Delta(1 / f)\right)^{2}+\left(\frac{\lambda z}{\pi w_{1}}\right)^{2}
\end{aligned}
$$

where $\lambda$ is the wavelength of the probe beam in air. Equations (4.33) and (4.34), in accordance with the thin lens approximation, presume that the thermal lens does not affect the width of the probe beam at the sample. Define the "thermal lens signal" $S(t)$ as the relative change of far-field axial intensity as a function of elapsed time $t$ following the arival of the pump pulse:

$$
S(t)=\frac{\left(I_{2}^{\prime}-I_{2}\right)}{I_{2}^{\prime}}
$$

From Eqs. (4.33)-(4.35), one has

$$
S(t)=\frac{z\left(1+z / R_{1}\right) \triangle(1 / f(t))-z^{2}(\Delta 1 / f(t))^{2}}{\left(1+z / R_{1}\right)^{2}+\left(\lambda z / \pi w_{1}^{2}\right)^{2}}
$$

Apart from the diffractive term in the denominator (viz. $\left.\left(\lambda z / \pi w_{1}^{2}\right)^{2}\right)$, Eq. (4.36) is the same as in the geometrical model. When the thermal lens is weak (i.e., when 
$\left.\triangle(1 / f) \ll 1_{i}^{\prime} R_{1}\right), S^{\prime}(t)$ is linear in $\triangle(1 / f)$ and may be decomposed into a sum of terms of the form

$$
S^{(m)}(t)=\frac{2 \pi}{\lambda} w_{1}^{2} \Delta(1 / f)^{(m)} \frac{B}{1+B^{2}}
$$

where

$$
B=\left(1+\frac{z}{R_{1}}\right) \frac{\pi w_{1}^{2}}{\lambda z}
$$

The parameter $B$ is a beam divergence parameter that becomes constant at large $\boldsymbol{z}$.

Equation (4.37) shows the effect upon a Gaussian probe beam of a thin, weak thermal lens that is caused by m-photon absorption. From Eqs. (4.28) and (4.31) we may write the thermal lensing signal as follows:

$$
S(t)^{(m)}=\left[\frac{2 \pi}{\lambda} \frac{\partial(n \ell-\ell)}{\partial T} \frac{(1-\eta) \beta^{(m)} h^{(m)} \Gamma_{0}^{m}}{C}\right] \frac{B}{1+B^{2}} \frac{4 m\left(w_{1}^{2} / w_{p}^{2}\right)}{\left(1+\left(8 m D t / w_{p}^{2}\right)\right)^{2}} .
$$

The term in square brackets is the initial thermally induced on-axis phase change of the probe beam, $\phi_{0}^{(m)}$. That is,

$$
\phi_{0}^{(m)}=\frac{2 \pi}{\lambda} \frac{\partial(n \ell-\ell)}{\partial T} T_{0}^{(m)} .
$$

The quantity $\phi_{0}^{(m)}$ is independent of parameters related to the specific distribution of pump beam fluence such as the width of the pump beam $w_{p}$ or the width of the thermal lens $w_{p}\left(1+8 m D t / w_{p}^{2}\right) / \sqrt{m}$. The terms outside the square brackets in Eq. (4.39) depend upon the probe beam's divergence $B$, upon the relative sizes of the probe and pump beams, and upon time. That is, the terms outside the square brackets account for beam geometry and thermal diffusion. The total thermal lensing signal in the weak, thin lens approximation is the sum of all relevant $m-$ photon contributions like Eq. (4.39). Each term is a product of an axial phase delay times a beam geometry factor. 


\subsection{Spherical Aberration in a Pulsed Thermal Lens.}

Because .he curvature in the wings of a Gaussian function is opposite to the curvature at the senter, a Gaussian-shaped lens has spherical aberration, as Fig. 4-4 illustrates. The previous section and all previous treatments of pulsed, dual-beam thermal lensing neglect the wings of the thermal lens. When the pump beam is not much wider than the probe beam, the wings of the beams contribute measurably to the thermal lensing signal, as will be shown. Some workers have considered aberrations in the case of a $\mathrm{cw}$, single-beam thermal lensing experiment, ${ }^{31}$ but the $\mathrm{cw}$ theory differs quantitatively from a theory suitable for pulsed, dual-beam thermal lensing. First, in the single-beam case, the diameter of only one beam need be considered. Second, and more importantly, the temperature distribution differs spatially and temporally for a continuous pump beam, compared to a short, pulsed beam. Consequently, the "shape" of the thermal lens is different in the $\mathrm{cw}$, single-beam case than it is in the pulsed, dual-beam case.

In this section, we treat the propagation of a Gaussian beam through a thin, weak Gaussian-shaped lens. We use the scalar theory of diffraction, treating the lens with a phase term in the Fresnel integral. Because of the weak-lens approximation, the exponential phase factor may be represented as a truncated power series. The axial intensity of the probe beam in the plane of observation is calculated as the square of the diffracted field strength.

For a circularly symmetric beam centered on a circularly symmetric thin lens the Fresnel integral is ${ }^{31}$

$$
\begin{aligned}
U_{2}(r)=\frac{2 \pi i \exp \left(-i k z-i k r^{2} / 2\right)}{\lambda z} \int_{0}^{\infty} & r^{\prime} d r^{\prime} J_{0}\left(\frac{k r r^{\prime}}{z}\right) \\
& \times U_{1}(r) \exp (-i \phi(r)) \exp \left(\frac{-i k r^{\prime 2}}{2}\right)
\end{aligned}
$$

where $J_{0}$ is a Bessel function, $U_{1}\left(r^{\prime}\right)$ is the complex-valued electric field of the probe 


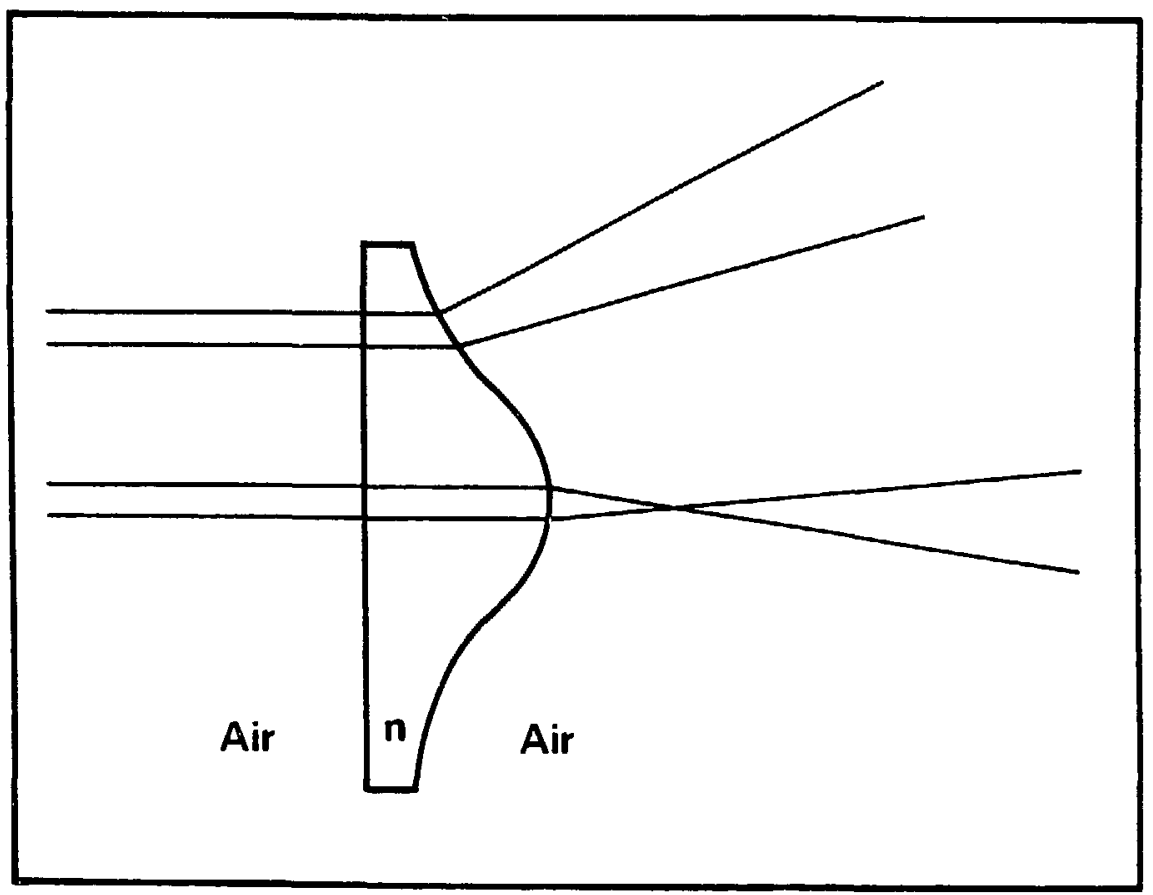

Figure 4-4. Lens with a Gaussian-Shaped Profile. Two beams are shown entering the lens from the left. The top beam passes through the lens where the curvature is negative, and so it diverges. The other beam passes through the lens where the curvature is positive, and so it focuses. 
beam in plane 1 containing the thin lens, $U_{2}(r)$ is the field in plane 2 a distance $z$ from the thin lens, $r^{\prime}$ and $r$ are the transverse radial coordinates in planes 1 and 2 , respectively, $\lambda$ is the optical wavelength, $k$ is $2 \pi / \lambda$, and $\phi\left(r^{\prime}\right)$ is the phase contributed by the lens to the field $U_{1}\left(r^{\prime}\right)$. For a Gaussian field and for a Gaussian lens profile, one has the following expressions: ${ }^{30}$

$$
\begin{aligned}
U_{1}\left(r^{\prime}\right) & =U_{1}(0) \exp \left(-\left(\frac{r^{\prime}}{w_{1}}\right)^{2}-\frac{i k{r^{\prime}}^{2}}{2} R_{1}\right) \\
\phi\left(r^{\prime}\right) & =\phi(0) \exp \left(-\left(\frac{r^{\prime}}{s}\right)^{2}\right)
\end{aligned}
$$

where $w_{1}$ and $1 / R_{1}$ are the initial beäm width parameter and phase curvature of the probe beam, $s$ is the $1 / e$ half-width of the thermal lens, and $\phi(0)$ is the total phase retardation at the center of the thermal lens. Expanding the exponential as a power series in $\phi$ and interchanging the order of summation and integration casts Eq. (4.41) into a sequence of recognizable integrals. ${ }^{33}$ For a weak lens the power series may be truncated after the first-order term. (One could choose to expand to higher order at the expense of much algebra, little insight, and not necessarily more accuracy.) Thus, one has

$$
\exp (-i \phi)=\mathbf{i}-i \phi
$$

Solving Eq. (4.41) as a function of arbitrary $r$ and multiplying the result by its complex conjugate yields an equation for the probe beam intensity at any point in plane 2. For $r=0$ the Fresnel integral is readily solved by changing variables to $x=r^{2}$ with the result that

$$
I_{2}=I_{2}\left(2+2 \phi(0) G_{1}+\phi^{2}(0) G_{2}\right)
$$

As before, $I_{2}$ is the axial intensity in plane 2 in the absence of a thermal lens. The coefficients $G_{1}$ and $G_{2}$ in Eq. (4.45) depend upon $B$, the diffractive broadening 
parameter defined in Section 4.2, as well as upon $s / w_{1}$, the ratio of lens width to probe beam width at the lens.

$$
\begin{aligned}
& G_{1}=\frac{B\left(s / w_{1}\right)^{2}}{\left(1+\left(s / w_{1}\right)^{2}\right)^{2}+B^{2}} \\
& G_{2}=\frac{\left(s / w_{1}\right)^{4}\left(1+B^{2}\right)}{\left(1+\left(s / w_{1}\right)^{2}\right)^{2}+B^{2}}
\end{aligned}
$$

The Gaussian thermal lens for an m-photon absorption is given by the following substitutions:

$$
\begin{aligned}
\phi(0) & =\frac{\phi_{0}^{(m)}}{\left(1+2 m t / t_{c}\right)} \\
\phi_{0}^{(m)} & =\frac{2 \pi}{\lambda} \frac{\partial}{\partial T}(n \ell-\ell) T_{0}^{(m)} \\
s^{2} & =\frac{w_{p}^{2}}{2 m}\left(1+2 m t / t_{c}\right),
\end{aligned}
$$

where $t_{c}$ is the thermal diffusion time,

$$
t_{c}=\frac{w_{r}^{2}}{4 L}
$$

The initial on-axis temperature change $T_{0}^{(m)}$ is given by

$$
T_{0}^{(m)}=\beta^{(m)} h^{(m)} \Gamma_{0}^{m}(1-\eta) / C
$$

where all terms in Eq. (4.51) have been defined in the previous section. To lowest order in $\phi_{0}^{(m)}$ the transient thermal lens signal defined by

$$
S^{(m)}(t)=1-\frac{I_{2}(t)}{I_{2}(0)}
$$

is the following expression:

$$
S^{(m)}(t)=\phi_{0}^{(m)} \frac{4 m B w_{1}^{2} / w^{2}}{\left(1+2 m w_{1}^{2} / w_{p}^{2}+2 m t / t_{c}\right)^{2}+B^{2}\left(1+2 m t / t_{c}\right)^{2}} .
$$

In Eqs. (4.52) and (4.53) the time-dependence of the axial intensity $I_{2}(t)$ has been explicitly written. From Eq. (4.48) we may express $\phi_{0}$ in terms of the m-photon absorption coefficient as follows:

$$
\phi_{0}^{(m)}=\beta^{(m)} \frac{2 \pi}{\lambda} \frac{\partial}{\partial T}(n \ell-\ell) h^{(m)} \Gamma^{(m)}(1-\eta) / C .
$$


Equation (4.53) is the main result of this Chapter. It will be used in chapter 5 to analyze our measurements.

When one neglects aberrations due to the thermal lens, the expression for the transient thermal lens signal is, to first order in $\phi_{0}^{(m)}$, the same as derived in the previous section (cf. Eq. (4.39)):

$$
S^{(m)}(t)=\phi_{0}^{(m)} \frac{B 4 m \dot{w}_{1}^{2} / w_{p}^{2}}{\left(1+B^{2}\right)\left(1+2 m t / t_{c}\right)^{2}} .
$$

The two expressions for $S^{(m)}(t)$ in Eqs. (4.53) and (4.55) are approximately equal when the pump beam is much wider than the probe beam - that is, when $w_{p} / \sqrt{m}$ is much larger than $w_{1}$. The theories disagree measurably when the thermal lens diameter is equal to or less than the probe beam diameter. Figure 4-5 compares the theoretical predictions of this theory, Eq. (4.53), to the predictions of previous theory, Eq. (4.55), for a hypothetical case with $w_{1}=2 w_{p}=0.2 \mathrm{rnm}, \lambda=532 \mathrm{~nm}$, $z=1800 \mathrm{~mm}, R_{1}=130 \mathrm{~mm}$, and $m=1$. The signals have both been normalized to unity at $t=0$ in order to compare their time dependence.

By normalizing both curves to unity at $t=0$, one can see that the transient signal predicted by Eq. (4.53) takes considerably longer to decay than does the signal predicted by Eq. (4.55) (about five times longer in this example). The reason is, previous theories deal only with the time required for the temperature to dissipate across the initial area of the thermal lens, but this theory accounts for the time required for the temperature to dissipate across the area of the probe beam, too. Normalizing both curves to unity at $t=0$ also conceals the fact that Eq. (4.55) predicts an initial signal that is $\left(1+B^{2}\right) /\left(\left(1+2 m w_{1}^{2} / w^{2}\right)^{2}+B^{2}\right)=17.5$ times greater than the initial signal predicted by Eq. (4.54), given the values of $\mathrm{B}$ and $2 m w_{1}^{2} / w_{p}^{2}$ used in Figure 4-5. From these results, one can see that using the previous thermal lensing theory, Eq. (4.55), could easily lead to an error of a factor of 5 in determining $t_{c}$ and an error of a factor of 15-20 in inferring $\phi_{0}^{(m)}$ or $\beta^{(m)}$. 


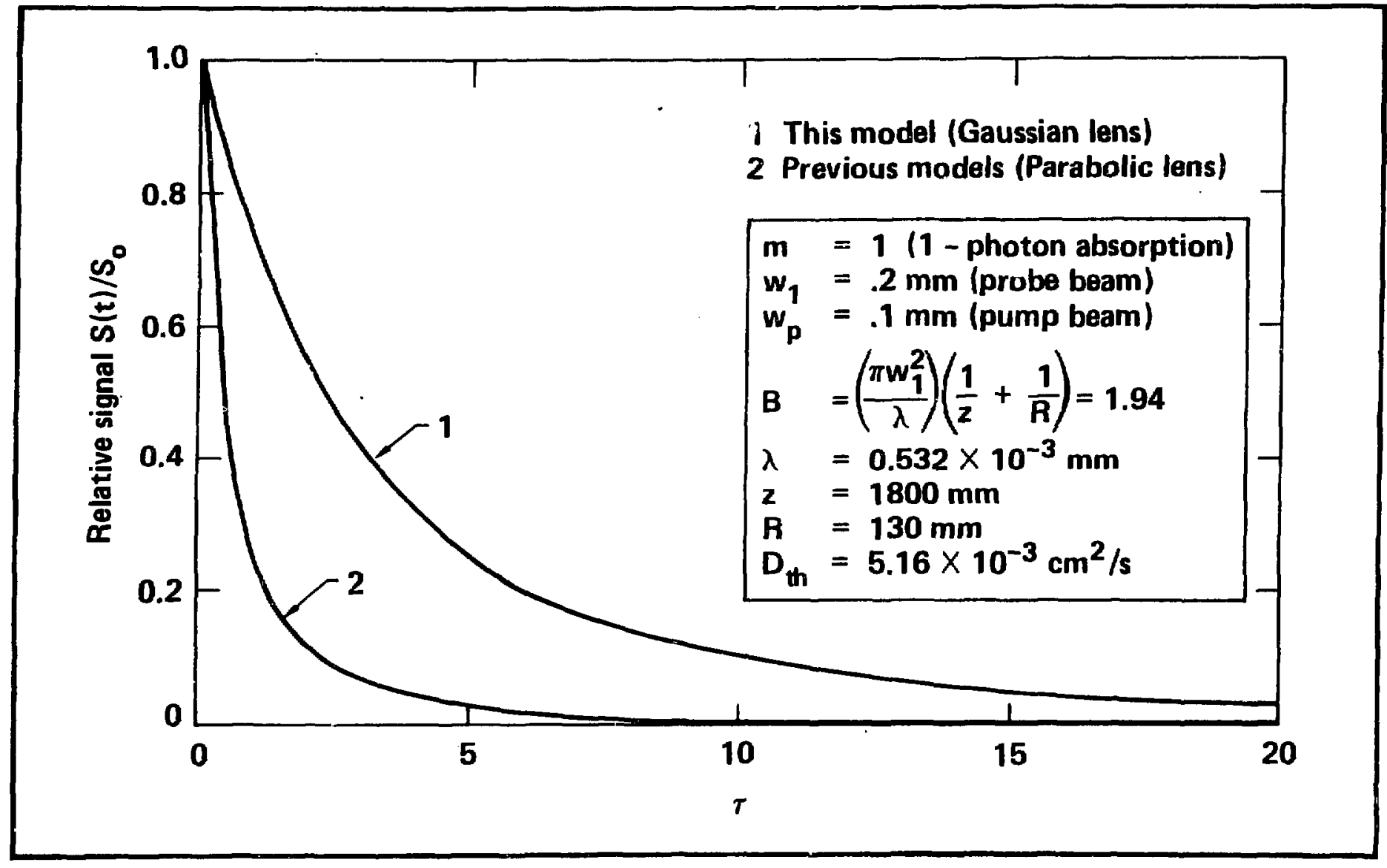

Figure 4-5. Comparison of Present Theory of Pulsed Thermal Lensing to the Previous Theory. Both curves have been normalized to unity at $t=0$. The dinensionless variable $\tau$ is defined as $\tau=2 \mathrm{mt} / t_{\mathrm{c}}$. 


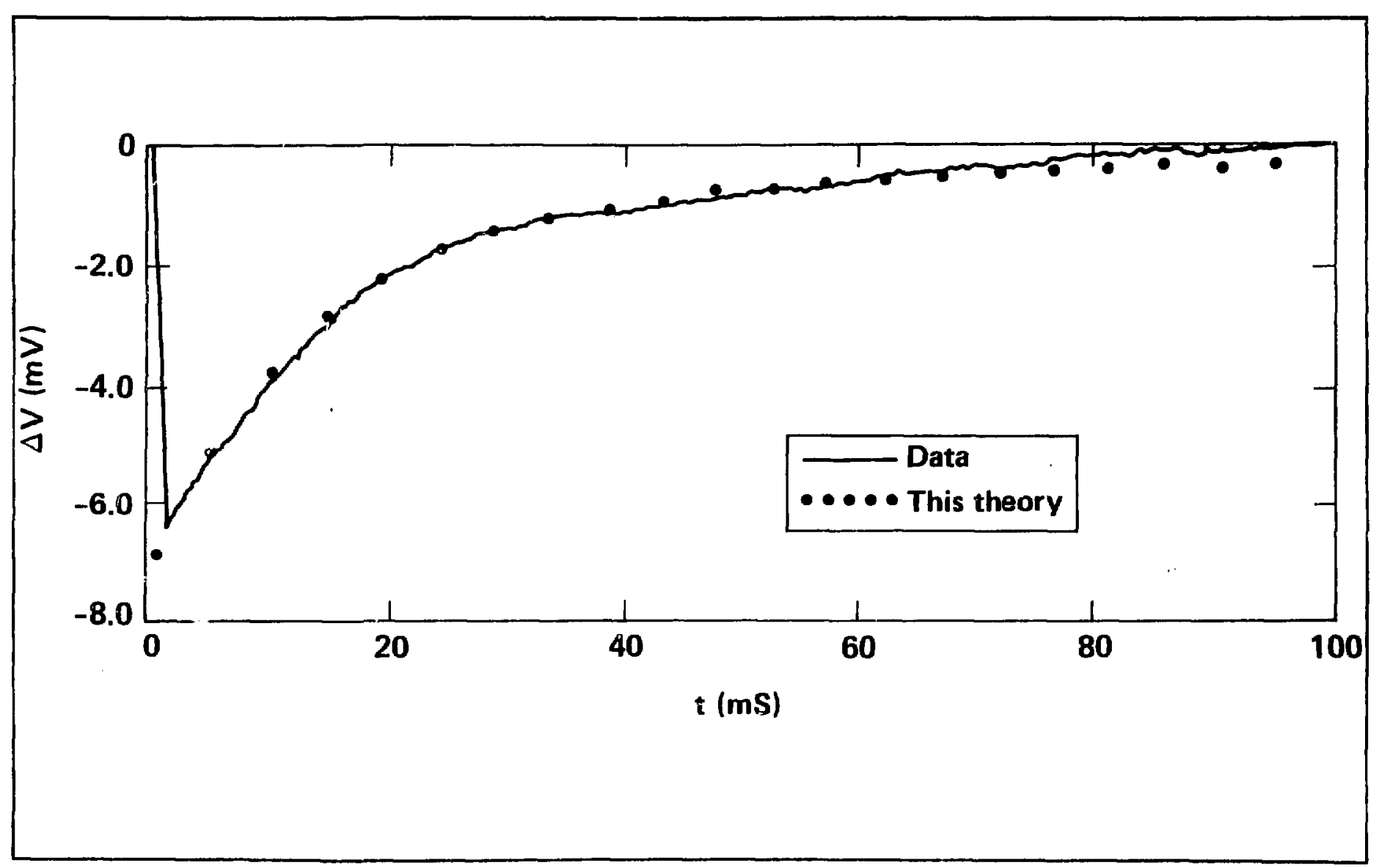

Figure 4-0. Comparison of Theoretical and Meisured Values of Thermal Lensing. 
Figure 4-6 compares actual thermal lensing data to the temporal shape predicted by our theory. The signal is an average of 100 pulses fired at a repetition rate of $10 \mathrm{~Hz}$. The sample is a 1-cm thick piece of solarized BK-7 glass. The pulsed beam is a 5-ns (FWHM) frequency-doubled Nd laser beam (Molectron MY-34). The probe beam is a cw HeNe laser beam (Spectra Physics 124B). The pulsed intensity has been kept low enough so that the peak thermal lensing signal is linear with applied fluence and therefore with $\phi_{0}^{(m)}$.

One can reduce the error associated with data analysis by using calibrated reference samples and by operating in the region where the thermal lensing signal is a linear function of the thermal lens strength, $\phi_{0}^{(m)}$. In the case of measuring peak signals $S_{0}$, operating in the "linear photothermal" region ensures that an unknown value $\phi_{0}^{(z)}$ for some sample can be determined from

$$
\phi_{0}^{(x)}=\phi_{0}^{(r e f)}\left(S_{0}^{(x)} / S_{0}^{(r e f)}\right) .
$$

Factors which involve only beam geometry (that is, those involving only $B$ and $2 m w_{1}^{2} / w_{p}^{2}$ ) cancel exactly in Eq. (4.56), assuming that the absorption is pureiy $m$-photon. In the case of measuring the thermal decay time $t_{c}$, beam geometry factors do not identically cancel. Still, when $B^{2}$ is small relative to $1+2 m w_{1}^{2} / w_{p}^{2}$, Eq. (4.53) approximates the form of Eq. (4.55) if $t_{c}$ is replaced by $t_{e f f}$, where

$$
t_{e f f}=t_{c}\left(1+2 m w_{1}^{2} / w_{p}^{2}\right)
$$

In summary, previous analyses of pulsed dual beam thermal lensing fail to account for the spherical aberration due to the wings of the thermal lens. These aberrations are important when the thermal lens is not several times larger than the probe beam diameter. We have accounted for these aberrations by expanding the term $\exp (-\mathrm{i} \phi)$ as a truncated power series in $\phi$ within the Fresnel integral. We have considered only the lowest order expansion, but it is straightforward to extend 


$$
-129-
$$

the analysis to higher orders if desired. Our theory agrees with experiment, as shown in Fig. 4-6. The inaccuracies of previous theories can be reduced if measurements are made relative to calibrated samples and if the thermal lensing signal is weak enough to be a linear function of the thermal lens amplitude, $\phi_{0}$. 


\section{References}

1. A. J. Twarowski and D. Kliger, "Multiphoton absorption spectra using thermal blooming," Chem. Phys. 20(2), 253-64 (North Holland Publishing Co., Amsterdam, 1 Mar. 1977).

2. W. B. Jackson, N. M. Amer, A. C. Boccara, and D. Fournier, "Photothermal deflection spectroscopy and detection," Appl. Opt. 20(8), 1333-44 (15 Apr. 1981).

3. W. T. White, III, M. A. Henesian, and M. J. Weber, "Photothermal lensing measurements of two-photon absorption and two-photon-induced color centers in borosilicate glasses at $532 \mathrm{~nm}$," to be published in J. Opt. Soc. Am. B 2(9), (Sept. 1985).

4. M. A. Henesian and M. J. Weber, "Measurement of nonlinear optical effects," in Laser Program Annual Review UCRL 50021-81, E. V. George, scientific editor, 7.31-2 (University of California, Lawrence Livermore National Laboratory, Aug. 1982).

5. M. E. Long, R. L. Swofford, and A. C. Albrecht, "Thermal lens technique: a new method of absorption spectroscopy," Science 19, 183-4 (16 Jan. 1976).

6. E. A. McLean, L. Sica, and A. J. Glass, "Interferometric observation of absorption induced index change associated with thermal blooming," Appl. Phys. Lett. 13(11), 369-71 (1 Dec. 1968).

7. Photoacoustic and Photothermal Spectroscopy, J. Badoz and D. Fournier (editors), J. Phys. (Paris) 44, Colloq. (C6), (Oct. 1983).

8. M. Bass, E. W. Van Stryland, and A. F. Stewart, "Laser calorimetric measurement of two-photon absorption," Appl. Phys. Lett. 34(2), 142-3 (15 Jan. 1979). 
9. M. A. Olmstead and N. M. Amer, "A new probe of the optical properties of surfaces," J. Vac. Sci. Technol. B1(3), 751-3 (July-Sept. 1983).

10. J. C. Murphy and L. C. Aamodt, "Photothermal spectroscopy using optical beam probing mirage effect," J. Appl. Phys. 51(9), 4580-8 (1980).

11. J. P. Gordon, R. C. C. Leite, R. S. Moore, S. P. S. Porto, and J. R. Whinnery, "Long-transient effects in lasers with inserted liquid samples," J. Appl. Phys. 36(1), 3-8 (Jan. 1965).

12. C. Hu and J. R. Whinnery, "New thermooptical measurement method and a comparison with other methods," Appl. Opt. 12(1), 72-9 (Jan. 1973).

13. H. L. Fang, T. L. Gustafson, and R. L. Swofford, "Two-photon absorption photothermal spetroscopy using a synchronously pumped picosecond dye laser. Thermal lensing spectra of naphthalene and diphenylbutadiene," J. Chem. Phys. 78(4), 1663-9 (15 Feb. 1983).

14. D. Magde, J. H. Brannon, T. L. Cremers, and J. Olmsted, III, "Absolute luminescence yield of cresyl violet. A standard for the red," J. Phys. Chem. 83(6), 696-9 (22 Mar. 1979).

15. G. Nolle, H. Ullrich, J. B. Muller, and J. Hesse, "A microprocessor controlled spectrometer for thermal scan Mossbauer ipectroscopy," Nucl. Inst. and Meth. in Phys. Res. 207(3), 459-63 (1 Apr. 1983).

16. G. Koren, "Thermal blooming measurement of specific heat and thermal conductivity in isotropic p-methoxybenzylidene-p-butylanoline (MBBA) near clearing," Phys. Rev. A 13(3), 1177-84 (1976).

17. M. C. Gupta, S.-D. Hong, A. Gupta, and J. Moacanin, "Thermal diffusivity measurements using a pulsed dual-beam thermal lens technique," Appl. Phys. Lett. 37(6), 505-7 (15 Sept. 1980).

18. A. Hordvik, "An experimental investigation of thermal distortion in infrared 
window materials," Conference on High Power Infrared Laser Window Materials, 27 and 28 Oct. 1971, C. S. Sahagian and C. A. Pitha, editors, AFCRL-710592, Special Report Number 127 (Air Force Cambridge Research Laboratory, Hanscom Field, Mass., 13 Dec. 1971); Y. Kohanzadeh, K. W. Ma, and J. R. Whinnery, "Measurement of refractive index change with temperature using thermal self-phase modulation," Appl. Opt. 12(7), 1584-87 (July 1973).

19. K. Darée, "Photochemical blooming of laser beams," Opt. Comm. 4(3), 238-42 (Nov. 1971).

20. B. S. H. Royce, F. Sanchez-Sinencio, R. Goldstein, R. Muratore, R. Williams, and W. M. Yim, "Studies of photocorrosion at the zinc-selenide-electrolyte interface by photothermal deflection spectroscopy," J. Electrochem. Soc. 129(10), 2393-5 (Oct. 1982).

21. C. A. Carter and J. M. Harris, "Thermal lens absorption measurements on small volume samples," Anal. Chem. 56(6), 922-5 (May 1984).

22. P. Calmettes and C. Laj, "Study of heat diffusion in transparent media by means of a thermal lens effect," Phys. Rev. Lett 27(5), 239-42 (2 Aug. 1971).

23. M. J. D. Low and A. G. Severdia, "Infrared spectra of a single human hair," Spectrosc. Lett. 16(11), 871-77 (Nov. 1983).

24. M. J. D. Low and C. Morterra, "IR studies of carbons I. IR photothermal beam deflection spectroscopy and II. The vacuum pyrolisis of cellulose," Carbon 21(3), 275-81 (Pergamon Press 1983).

25. M. Sparks, "Optical distortion by heated windows in high-power laser systems," J. Appl. Phys. 42(12), 5029-46 (Nov. 1971).

26. Nd-Doped Laser Glass Spectroscopic and Physical Properties, Handbook LLNLM-95, S. E. Stokowski, R. A. Saroyan, and M. J. Weber, editors, (University of California, Lawrence Livermore National Laboratory, Livermore, California, 
1978).

27. C. Kittel, Introduction to Solid State Physics, Fifth Edition (John Wiley and Sons, N. Y., 1975).

28. N. F. Mott and E. A. Davis, Electronic Processes in Non-Crystalline Materials, 233, (Clarendon Press, Oxford, 1971).

29. H. S. Carslaw and J. C. Jaeger, Conduction of Heat in Solid, Second Edition, (Clarendon Press, Oxford 1959).

30. A. Yariv, Quantum Electronics, 2nd Edition (John Wiley, New York, 1975).

31. S. J. Sheldon, L. V. Knight, and J. M. Thorne, "Laser-induced thermal lens effect: a new theoretical model," Appl. Opt. 21(9), 1633-9 (1 May 1982).

32. J. W. Goodman, Introduction to Fourier Optics (McGraw-Hill, San Francisco, 1968).

33. I. S. Gradshteyn and I. M. Ryzhik, Tables of Integrals, Series and Products (Academic Press, New York, 1965). 
Chapter 5

\section{EXPERIMENTAL RESULTS}

\subsection{Introduction.}

This chapter describes our thermal lensing measurements. Several new results are presented, including the first application of photothermal lensing to measure nonlinear absorption in a solid and nonlinear absorption measurements in several glasses. Section 5.1 details the experimental apparatus and procedure. Sections $\mathbf{5 . 2}$ and 5.3 discuss measurements in $\mathrm{ZnS}$ and $\mathrm{TiO}_{2}$, respectively. Section 5.4 treats the measurement of nonlinear absorption in glasses. Section 5.5 discusses the use of Nddoped samples to calibrate the measurements in the glasses. Section 5.6 considers our accuracy, and Section 5.7 summarizes our findings.

\subsection{Apparatus and Procedures.}

Figure 5-1 shows the optical layout of the experiment. A pair of orthogonally polarized pump and probe beams entered from the left. After passing through various optical elements, they merged at beam splitter BS1. From there, they passed collinearly through sample $\mathbf{S}$ and were spatially separated by a Wollaston prism, P2. Beyond prism P2, the isolated pump beam entered a beam dump, BD, and the probe beam passed through aperture A2 so that changes in its axial intensity could be detected. Photodiodes PD1, PD2, and PD3 all had optical filters (F1, F2, and F3, respectively), baffles, and black cloth to block stray light. A thermally compensated, zero-order half-wave plate, WP, and a Glan polarizer, P1, controlled the intensity of the pump beam. Rotating the waveplate did not steer the beam measurably. Adjustable aperture A1 clipped the pump beam, diffractively filling in the center of the beam at the sample. Rapid selection of the best diameter for aperture A1 was achieved by examining a real-time, color-coded, iso-intensity 


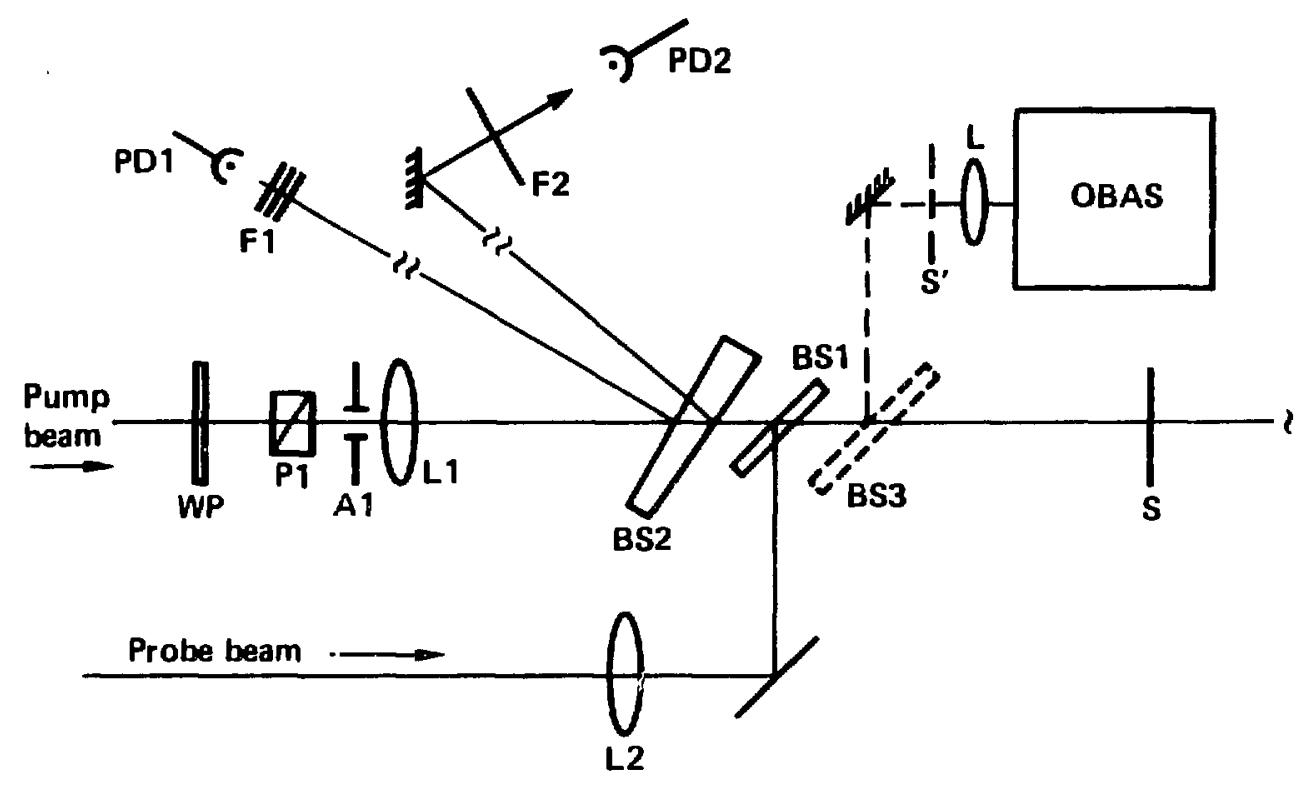

Figure 5-1. Photolhermal Lensing Experiment. Sce the text, for a discussion. 
contour map of the pump beam on the video display of the optical beam analysis system (OBAS). Much effort went into the OBAS, because traditionally a large inaccuracy in nonlinear optical measurements comes from a lack of knowledge of the pump beam's intensity. ${ }^{1}$ Beam splitters BS2 and BS3 picked off portions of the pump beam for measuring the energy $E$, the temporal pulsewidth $\tau$, and the relative fuence $\Gamma^{\prime}$. Beam splitter BS1 sat at Brewster's angle to minimize reflective losses of pump beam energy and to prevent stray reflections of pump beam energy from entering the probe laser. Beam splitter BS2 was tilted approximately $10^{\circ}$ from normal incidence and had a $4^{\circ}$ wedge in order to separate the reflections from its front and back surfaces. Beam splitter BS3 was kinematically mounted (as implied by the dashed lines in Fig. 5-1), and so it could be removed after measuring the beam profile with the OBAS. All the beam splitters were fused silica for high resistance to effects such as self-focusing and damage. Lenses L1 and L2 both had effective focal lengths of $50 \mathrm{~cm}$. The sample $S$ sat typically $3-5 \mathrm{~cm}$ in front of the waist of the probe beam. The distance from the sample to the aperture A2 was $195 \mathrm{~cm}$.

\subsubsection{The Pump Beam.}

The pump beam was a dye laser (Molectron DL-18) excited at a $10-\mathrm{Hz}$ repetition rate by a Q-switched, frequency-doubled Nd:YAG laser (Molectron MY-34-10). The Nd:YAG laser was an unstable resonator with an amplifier. The frequency-doubled $\mathrm{Nd}$ :YAG beam determined the primary spatial and temporal characteristics of the dye laser beam. Approximately $10 \%$ of the power of the frequency-doubled Nd:YAG laser beam was used to drive the plane-plane dye laser resonator, and the remaining energy powered the dye amplifier. The dye laser had a transversely pumped oscillator and a longitudinally pumped amplifier. An intra-cavity linear diffraction grating in the dye oscillator provided wavelength selectivity. 


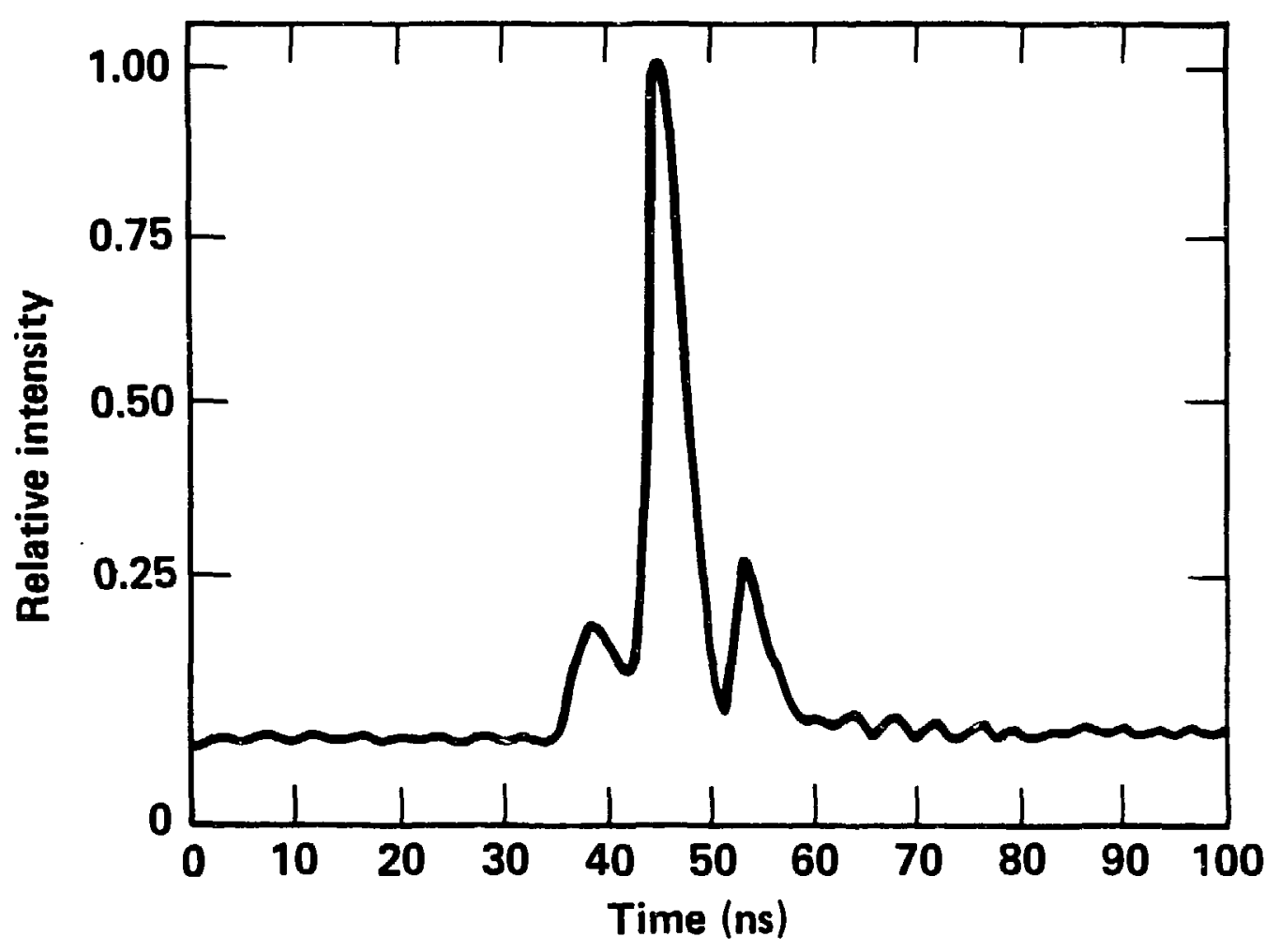

Figure 5-2. Temporal Pulse Shape of the Probe Beam at $532 \mathrm{~nm}$. 
Figure 5-2 shows the temporal pulse shape of the dye beam. A central lobe contained about $65 \%$ of the energy, two-side lobes contained about $25 \%$, and a long tail contained the remaining $10 \%$. The temporal structure was due to axial mode beating in the Nd:YAG oscillator. In general, the Nd:YAG oscillator was driven as hard as possible (short of damaging the laser rod) in order to maximize the intensity. The shot-to-shot fluctuations of the frequency-doubled Nd:YAG laser were typically less than $\pm 12 \%$ in amplitude, but irregularities in the laser dye due to bubbles, particles, and dye degradation decreased the pulse stability so that on a shot-to-shot basis, the peak amplitude of the dye laser varied by $\pm 27 \%$. The pulse-shape factor $h^{(2)}$ (cf. Eq. (4.23)) varied by $\pm 16 \%$ from shot-to-shot, but when averaged over 40 consecutive shots, $h^{(2)}$ varied only $\pm 4 \%$ from one sequence of consecutive shots to the next.

Figure 5-3 shows the spatial profile of the dye beam. The figure consists of five adjacent video line scans through the center of the beam in plane $S^{\prime}$, the sampleequivalent image plane. To a good approximation, a Gaussian function $\exp \left(-2 \mathrm{r}^{2} / \mathrm{w}^{2}\right)$ described the spatial distribution of energy. Heating in the television camera led to a non-uniform background video signal that gradually increased over a period of hours. The OBAS microcomputer subtracted this background digitally from the image of the beam prior to computing the relative fluence. The beam width and divergence of the dye laser beam changed from dye to dye. This necessitated measuring the beam profile at almost every wavelength. Although clipping the beam with aperture Al reduced the spatial irregularities in the pump beam, half of the energy was lost by spatial filtering. At the sample the spatial jitter of the pump beam was about $10-20 \%$ of the pump beam width $w_{p}$. 


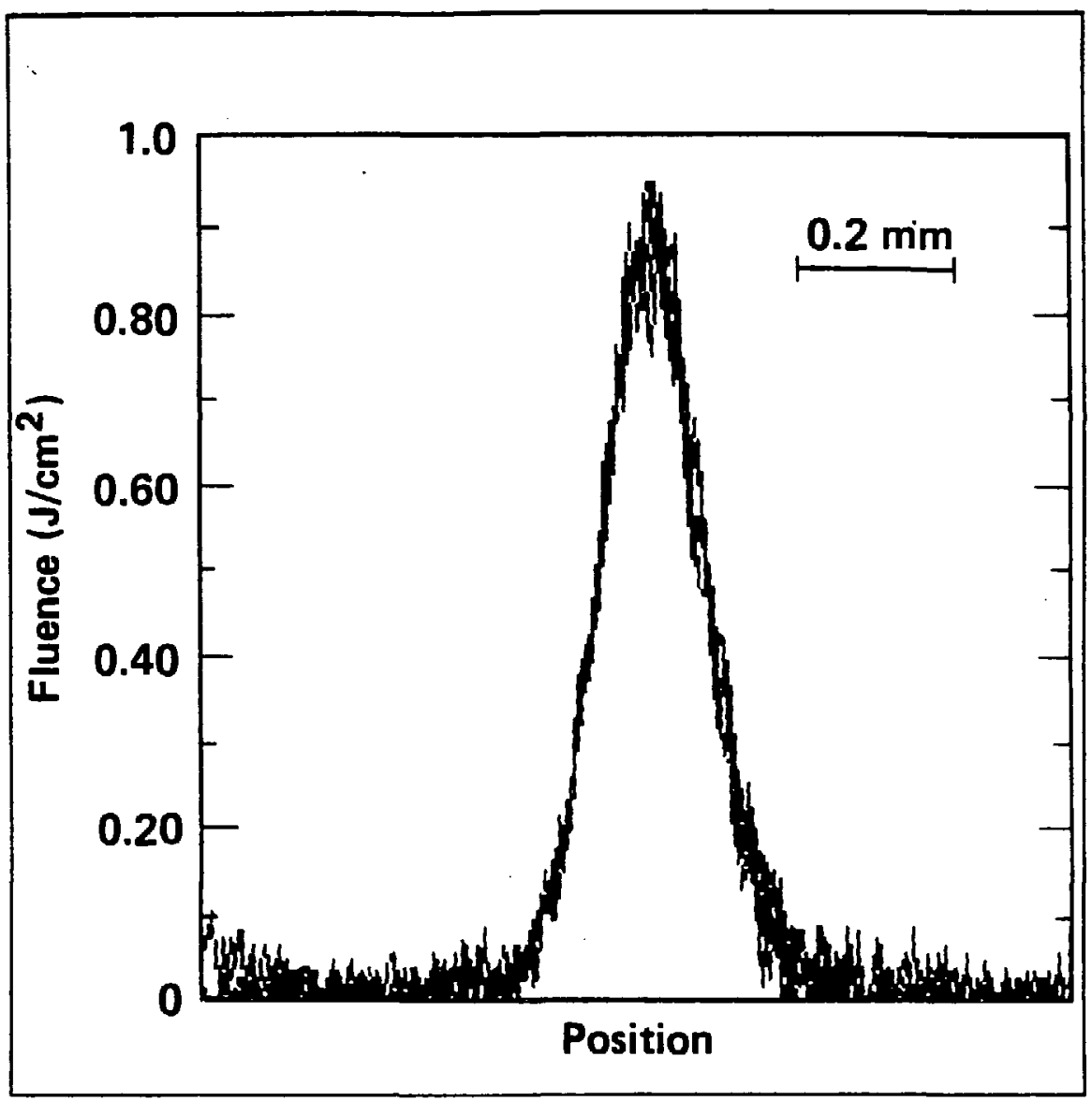

Figure 5-3. Distribution of Fluence in the Pump Beam. The beam is very nearly Gaussian. The graph represents five adjacent line scans through the center of the digitized video image of the beam. The responsivity of the video tube is flat to within $5 \%$ at the center, where the beam hits. The non-uniform background due to heat build-up in the vidicon has been subtracted from the signal. 


\subsubsection{The Probe Beam.}

A continuous wave, 24-mW HeNe laser (Spectra Physics 124B) provided the probe beam. The laser had a low-ripple power supply and special optical mounting hardware for achieving temporally constant output power. Power supply leakage at harmonics of $60 \mathrm{~Hz}$ and random lasing fluctuations contributed a measured $0.3 \%$ rms noise to the probe beam intensity on a time scale of seconds. Over periods of hours, the drift in laser intensity was insignificant. Figure 5-4 shows the spatial profile of the probe beam. Like that of the pump beam, this profile was described well by a Gaussian function.

The Gaussian beam waist size and location were determined by measuring the encircled power as a function of axial position. Encircled power is the power that is transmitted by a circular aperture centered on the optical axis. If $P_{t o t}, P$, and $w_{1}$ respectively denote the total power, the encircled power, and the beam width in a plane that is a distance $z_{1}$ from the beam waist, then for a Gaussian beam profile one has

$$
P=P_{t o t}\left(1-\exp \left(\frac{-2 a^{2}}{w_{1}^{2}}\right)\right)
$$

where $2 \mathrm{a}$ is the diameter of the aperture. The encircled power $P$ reaches its maximum where the spot size is a minimum, namely at $z_{1}=0$. The spot size $w_{1}$ varies with axial position $z_{1}$ according to the relation ${ }^{2}$

$$
w_{1}^{2}=w_{0}^{2}\left(1+\frac{z_{1}^{2}}{z_{0}^{2}}\right)
$$

where $w_{0}$ is the beam waist size and $z_{0}$ is the so-called "Rayleigh distance" or "confocal beam parameter." The latter is expressed in terms of $w_{0} \mathrm{as}^{2}$

$$
z_{0}=\frac{\pi w_{0}^{2}}{\lambda}
$$

where $\lambda$ is the optical wavelength. Knowing the position and size of the beam waist, 


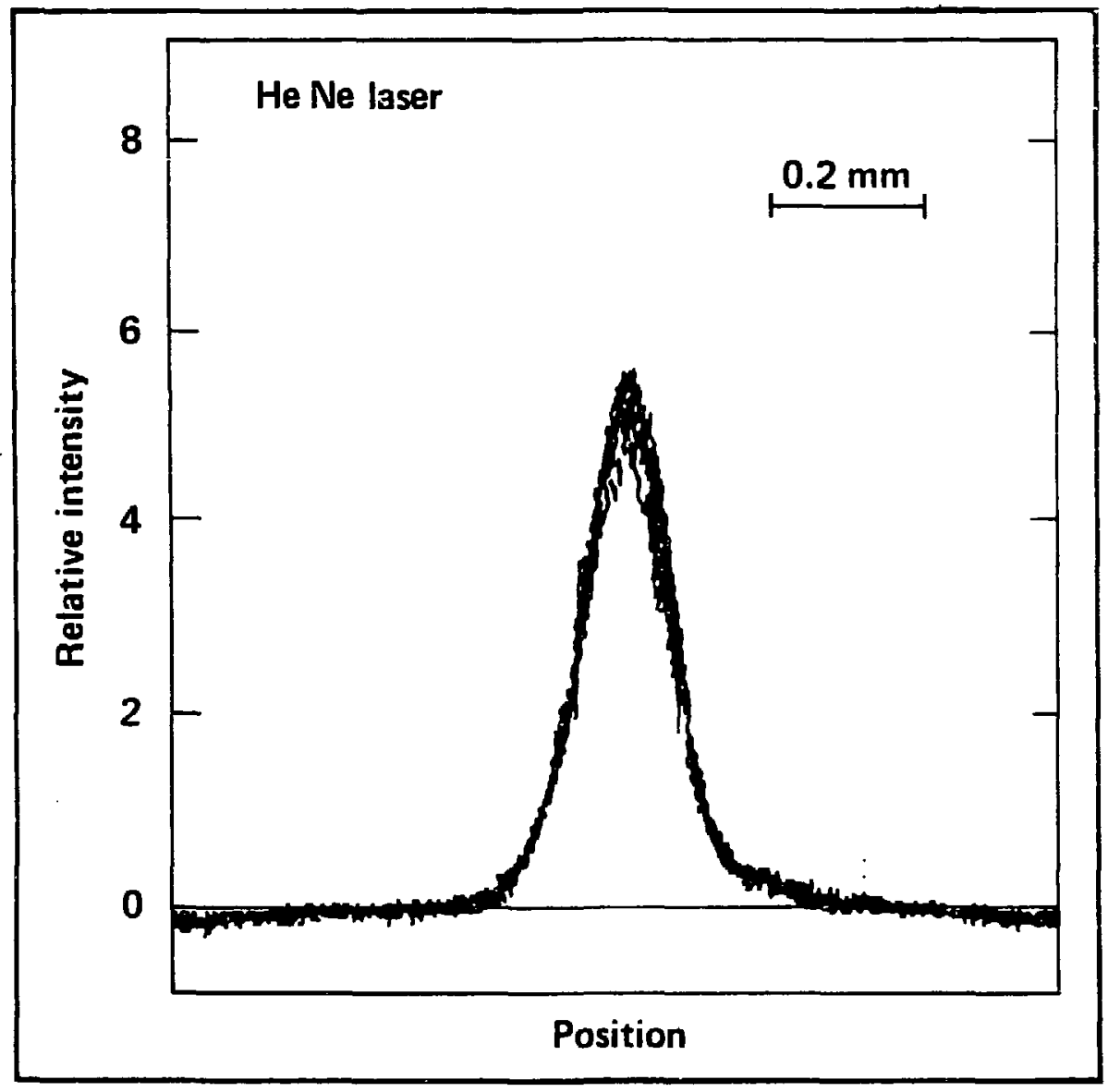

Figure 5-4. Spatial Profile of the HeNe Probe Beam. 
one obtains the radius of curvature of the phase front at any loc ation $z_{1}$ from $^{2}$

$$
R_{1}=-z_{1}\left(1+\frac{z_{0}^{2}}{z_{1}^{2}}\right)
$$

The sign convention is chosen so that $R_{1}$ is positive when the beam diverges at $z_{1}$ and so that $z_{1}$ increases in the direction of propagation.

Figure 5-5 shows the values of $\mathrm{P} / \mathrm{P}_{\text {tot }}$ as a function of position for the HeNe probe beam $(\lambda=0.6328 \mu \mathrm{m})$ measured with a $50-\mu \mathrm{m}$ diameter aperture. From those data, $z_{0}$ was estimated as $2.8 \mathrm{~cm}$.

\subsubsection{Photodetectors and Signal Averagers.}

A variety of photoconductive silicon diodes monitored both the pump and the probe beams. Because the thermal lensing signals were weak, signal averaging techniques were generally used to improve the signal-to-noise ratio. Signal averagers that were used included multichannel scalers, lock-in amplifiers, and boxcar averagers. In some cases, single-shot measurements had to be made, because the optical properties of some samples changed from shot to shot due to solarization. ${ }^{3}$

\subsubsection{Optical Beam Analysis System.}

A video digitizer connected to a microcomputer enabled us to determine the relative fluence distribution in the laser beams. Figure 5-6 shows a block diagram of the system. A magnified image of the beam in the effective sample plane $S^{\prime}$ was projected by a lens onto the surface of a vidicon (RCA 4532U). A black and white television camera (Telemation TMC1100) rastered the diode array at $60 \mathrm{~Hz}$ with a standard American format of 525 interlaced horizontal lines and relayed the analog signal to a video digitizer D (Grinnell GMR 27-10). The digitizer operated under computer control (LSI-11/23) with a direct memory access (DMA) interface. The video digitizer performed a several related tasks such as relaying real-time images to a color television monitor (TV, in Fig. 5-6), digitizing and averaging incoming pictures, and transmitting digitized images to and from the computer. 


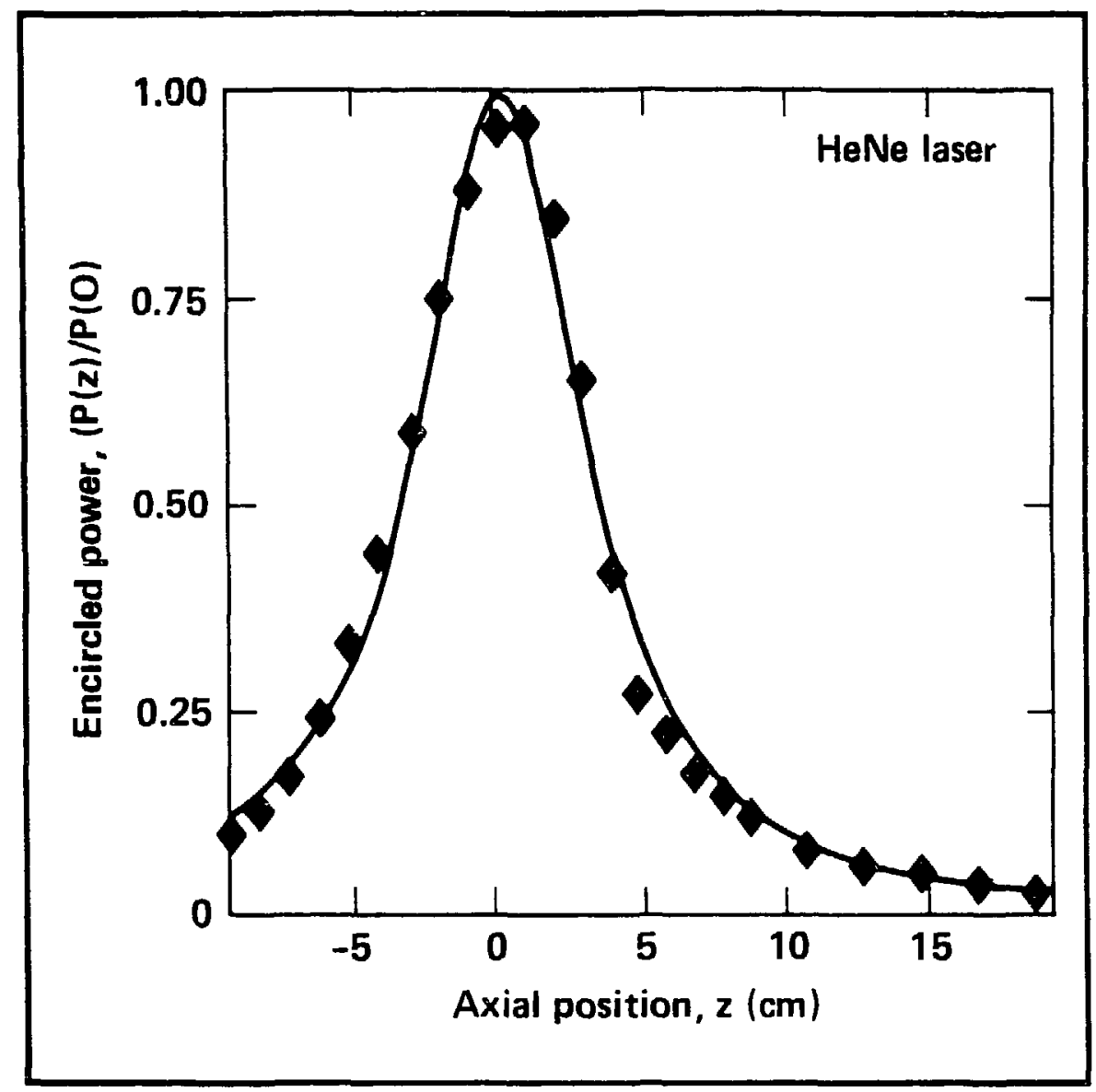

Figure 5-5. Encircled Power in the $\mathrm{HeNe}$ Probe Beam versus Axial Position. Dots represent measured values, and the solid curve is a fit using Eq.(5.1). 


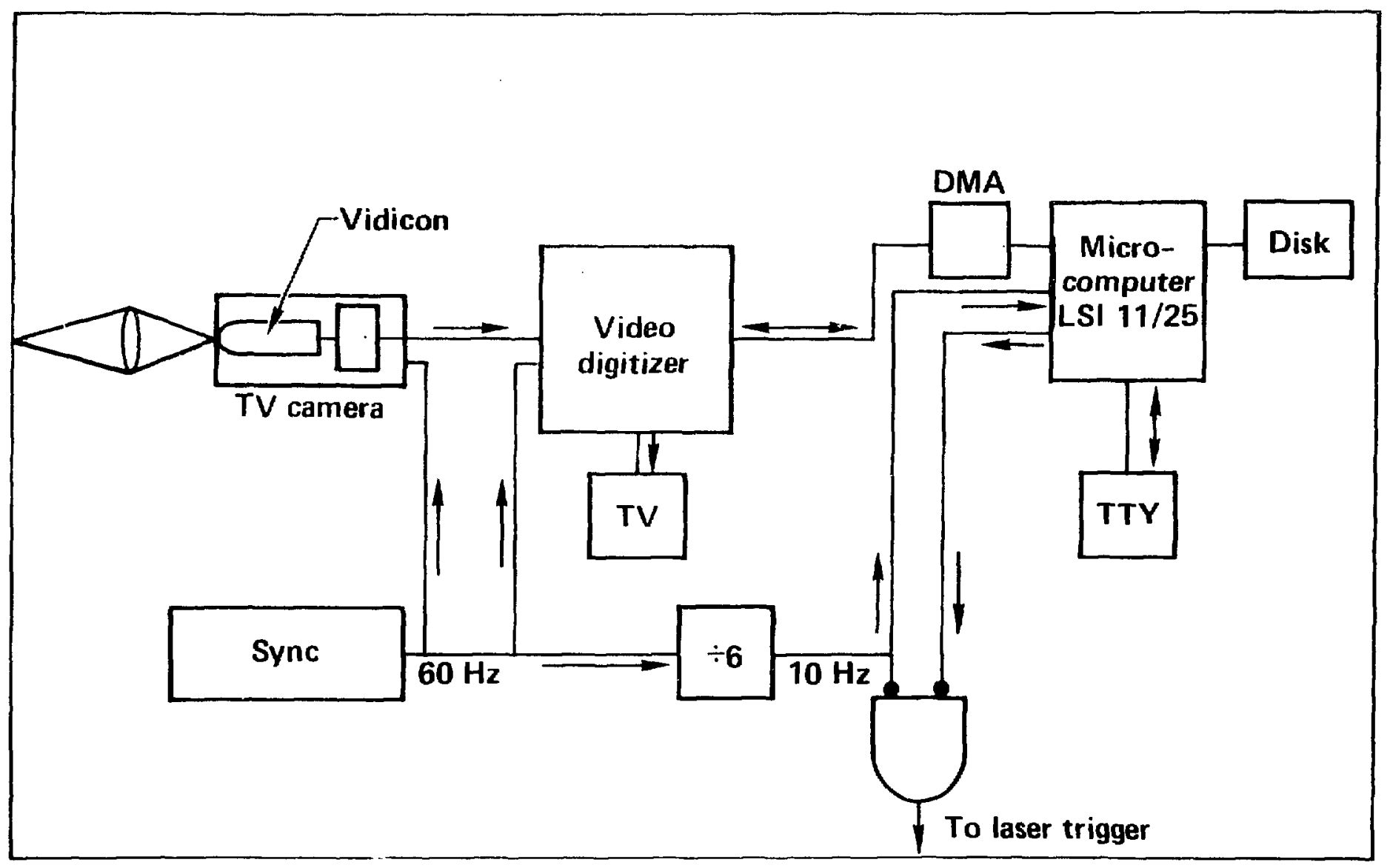

Figure 5-6. Optical Beam Analy'sis System (OBAS). 
The computer processed the digitized images to provide relative fluence and beam profile information. Results could be stored on a magnetic disk.

The signal linearity and spatial uniformity of the vidicon were checked with an "Optoliner", an incandescent illuminator that was uniformly bright over its field of view and that projected selected video test patterns directly onto the vidicon tube in the television camera. The results of the Optoliner measurements showed that the response of the vidicon was constant to better than $5 \%$ over the central active area of the vidicon tube, as long as the output video signal did not exceed about $87 \%$ of the maximum 0.7 volts. The automatic gain and peak-clipping circuits of the television camera were disabled for the sake of obtaining video signals that were proportional to the incident fluence.

Geometric image distortions were also checked with the Optoliner. They were negligible over the area of the laser beam footprint. A precision graticle that was reticulated on 0.1-mm centers provided spatial calibration for the OBAS. Placed at the equivalent sample plane $\mathbf{S}^{\prime}$, this graticle also served as a focusing aid for the relay lens $\mathrm{L}$ that imaged $\mathrm{S}^{\prime}$ onto the vidicon tube.

\subsubsection{Transient Digitizer.}

A transient-signal digitizer (Tektronix R7912) equipped with a rapid time base (7B92A) and a 500-MHz bandwidth amplifier (7A19) recorded the temporal pulseshape data of the dye laser beam. The transient digitizer was connected to a minicomputer with a disk drive (Tektronix CP4165/CP112) that processed and stored the digitized data. The temporal response of the transient digitizer and detector P1 were checked as a unit. Laser pulses that were nominally $300 \mathrm{ps}$ long and whose duration had previously been measured by optical correlation ${ }^{4}$ impinged upon the detector. The total electronic system broadened the pulses to $1.2 \mathrm{~ns}$ (FWHM). This was much less than the scale of observed temporal fluctuations in 
the dye laser bearn, which was 5-8 ns.

\subsubsection{Electronic Timing.}

A crystal-controlled clock synchronized the experiment. The clock was interfaced to the LSI-11/23 microcomputer so that we could control the experiment from the computer, if desired. The timing circuitry provided a $10-\mathrm{Hz}$ trigger pulse to the laser, a TTL pretrigger pulse to the various signal averagers $1.0 \mathrm{~ms}$ before the laser fired, and horizontal and vertical sync signals to the video equipment. The timing was adjusted so that the television camera was not rastering (i.e., $\mathbf{w}^{*}$ : internally "blanked") when the laser fired. A pulse counter controlled the number of consecutive laser pulses that were fired into the sample, and a divide-by- $\mathrm{N}$ circuit controlled the rate at which the laser fired. The time between consecutive pulses was $0.1 \times N$ seconds, where $\mathrm{N}$ was set by the user to $1,2,3,5,10,20,50$, or 100 . This capability allowed us to perform rate-dependent measurements to see if absorption varied noticeably as a function of delay between shots. It did not.

\subsubsection{Optical Energy Calibration.}

A pyroelectric joulemeter (Laser Precision Rj7200) calibrated photodiode PD1. (See Fig. 5-1.) During calibration, the joulemeter sat directly in the expanded main beam of the pump laser about a meter beyond the sample plane $S$, with the sample removed. The photodiode was calibrated directly in units of $\mathrm{mV}$ per $\mathrm{mJ}$ incident at the sample. In order to obtain the responsivity curve, one hundred shots were averaged at each energy level, and the energy of the pump beam was varied over its entire range. At the higher energies, the responsivity of photodiode PD1 saturated slightly, but this was accounted for in the analysis of the data. The pyroelectric detector was checked against a volumetric absorbing glass calorimeter (Scientech 361 ). Both detectors gave the same reading to within $15 \%$, regardless of optical wavelength. The uncertainty in the energy calibration was taken as $\pm 15 \%$. 


\subsubsection{Optical Alignment.}

The pump and probe laser beams were carefully overlapped in the sample in order to avoid thermal deflections ${ }^{5}$. First, with no sample in place the two beams were overlapped at two locations that were separated by about two meters and that were on opposite sides of the sample plane $S$. This made the beams parallel to within $1.0 \mathrm{mrad}$. Then a $1-\mathrm{mm}$ thick absorbing sample was placed at $\mathrm{S}$, and minor residual errors in beam overlap were removed by laterally adjusting lens L1 with a micrometer to maximize the thermal lensing signal. These small lens movements did not significantly alter the parallelism of the pump and probe beams. When the two beams were concentric at the sample the thermal lensing signal was strongest. As the pump beam was laterally displaced in any direction, the signal would fall, reverse sign, pass through a relative minimum, and then rise to 0 with increased displacement, making it easy to recognize when the pump beam was centered on the probe beam.

In the absence of a sample, no thermal lensing signal was observed, indicating that false signals due to lensing in other optical elements did not occur.

\subsubsection{Photothermal Linearity.}

In order for the theory of the previous chapter to apply, the thermal lensing signal must vary in direct proportion to the absorbed energy. That is, one must operate within a region of "photothermal linearity." This region was determined by using a linear absorbing sample of known absorbance and of known luminescent efficiency. We found that the thermal lensing signal was linear for signal up to $|\triangle I / I|=6 \%$. (In some cases the region of photothermal linearity extended to $40 \%$, but this was inexplicably high.) Since the nonlinear absorption measurements to be discussed in the following sections rarely resulted in changes of intensity that were greater than $2 \%$, the assumption of small signals was justified. Hence, the analysis 
of Chapter 4 applied.

The linearity of the thermal lensing signal was also checked as a function of the sample thickness for a linearly absorbing material. For a given material, the thermal lensing signal was proportional to the thickness of the sample, to within experimental uncertainty. This shorod that, within the limits of the experiment, surface effects could be neglected with respect to bulk effects, and that the thin lens approximation was satisfactory.

\subsubsection{Data Reduction.}

We analyzed our results in the following way: As in Chapter 4, let $S_{0}$ denote the thermal lensing signal immediately after the arrival of a pulse of the pump beam. $S_{0}$ is the fractional change of the far-field axial intensity of the probe beam due to thermal lensing. Equivalently, $S_{0}$ is the fractional change $\Delta V / V$ of the output of photodiode PD3 in Figure 5-1. For a sample with both linear and two-photon absorption, $S_{0}$ is a parabolic function of $\Gamma_{0}$, the peak fluence of the pump beam at the sample plane:

$$
S_{0}=A_{1} \Gamma_{0}+A_{2} \Gamma_{0}^{2} .
$$

Measuring $S_{0}$ at several fluences and fitting a curve of the form of Eq. (5.5) to the data yields the coefficients $A_{1}$ and $A_{2}$. From these two values one can estimate the one- and two-photon absorption coefficients, $\alpha$ and $\beta$, respectively. Accounting for the finite width of the Gaussian pump beam, Eqs. (4.53) and (4.54) show that

$$
\alpha=A_{1}\left[\frac{2 \pi}{\lambda} \frac{(1-\eta)}{C} \frac{\partial}{\partial T}(n \ell-\ell) \frac{4 B\left(w_{1} / w_{p}\right)^{2}}{\left(1+2 w_{1}^{2} / w_{p}^{2}\right)^{2}+B^{2}}\right]^{-1}
$$

and

$$
\beta=A_{2}\left[\frac{2 \pi}{\lambda} h^{(2)} \frac{(1-\eta)}{C} \frac{\partial}{\partial T}(n \ell-\ell) \frac{8 B\left(w_{1} / w_{p}\right)^{2}}{\left(1+4 w_{1}^{2} / w_{p}^{2}\right)^{2}+B^{2}}\right]^{-1} .
$$

Chapter 4 defined the parameters that appear in Eqs. (5.6) and (5.7). The effective thermal conversion efficiency is $1-\eta ; T$ is temperature; $\ell$ is sample thick- 
ness; $n$ is the refractive index of the sample at the wavelength of the probe, $\lambda ; w_{1}$ is the Gaussian half width of the probe beam at the sample; and $w_{p}$ is the corresponding half width of the pump beam. The pulse shape factor $h^{(2)}$, defined by Eq. (4.23), is the ratio of the square of the pump beam intensity integrated over the total duration of the pulse and divided by the square of the fluence. The beam diffraction parameter $B$, defined by $\mathrm{Eq} .(4.38)$, is

$$
B=\left(\frac{\pi w_{1}^{2}}{\lambda}\right)\left(\frac{1}{R_{1}}+\frac{1}{z}\right),
$$

where $R_{1}$ is the radius of curvature of the wavefront of the probe beam at the sample and where $z$ is the distance from the sample to the plane of observation, aperture A2 in Figure 5-1.

\subsection{Results for $\mathrm{ZnS}$.}

The thermal lensing measurements in this work were performed on chemically vapor deposited $\mathrm{ZnS}$ that was supplied by CVD, Inc. The manufacturer specially treated the material in a proprietary non-chemical process so that it was colorless and only slightly scattering. Prior to the treatment, the material was yellow and highly scattering. A 2.0-mm thick slab was cut and polished on opposite faces. $\mathrm{X}$-ray tests verified that the material was predominantly cubic with the (111) axes of the crystallites oriented approximately normally to the faces of the slab. Table 5-1 gives the material parameters that were used to analyze the thermal lensing data.

The experimental set-up as described in Section 5.1 was modified slightly. First, to reduce etalon effects, the uncoated sample was mounted at Brewster's angle. Then the pump beam and the probe beam were adjusted to be linearly polarized with both polarizations parallel. The pump beam was spectrally filtered out of the probe beam with narrow-band-pass $0.6328 \mu \mathrm{m}$ filters that were placed immediately in front of the photodiode PD3. The wavelength of the pump beam was varied from 
Structure $^{a} \quad \mathrm{~T}_{d^{2}}(\mathrm{~F} \overline{4} 3 \mathrm{~m})$

Density $(\mathrm{g} / \mathrm{cc})^{c} \quad \rho=4.01$

Specific Heat at $300 \mathrm{~K}(\mathrm{~J} / \mathrm{cc} / \mathrm{K})^{b} C_{p}=1.84$

Thermal Coefficient of Linear Expansion $\left(10^{-6} K^{-1}\right)^{c} \alpha_{t h}=4.6$

Refractive Index at $0.6328 \mu \mathrm{m}^{b} n=2.36$

Thermo-optic coefficient at $0.6328 \mu \mathrm{m}^{b}\left(10^{-6} K^{-1}\right) \partial n / \partial T=25$

Table 5-1. Physical Properties of $Z n S$ (Zinc Blende)

Ref. 6

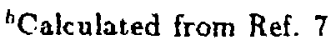

"heasured, Courtesy T. Hoheisel, Lawrence Livermore National Laboratory 
$0.55 \mu \mathrm{m}$ to $0.70 \mu \mathrm{m}(4.5 \mathrm{eV}>2 \hbar \omega>3.5 \mathrm{eV})$, and the thermal lensing signal was measured as a function of pump beam fluence.

Figure 5-7 shows the thermal lensing signal in $\mathrm{ZnS}$ at a given wavelength as a function of energy per pulse. The measured values lie along a parabola, indicating the occurrence of two-photon absorption. The slope of the curve at a given fluence is proportional to the effective coefficient of absorption at that fluence. At the origin the slope appears to vanish, showing that the intrinsic linear absorption coefficient is zero. At the wavelength shown, absorption is therefore nonlinear.

Each measurement in Figure 5-7 represents an average response of the sample to many consecutive pump pulses at a $10-\mathrm{Hz}$ repetition rate. The actual number of shots that was taken per measurement depended upon the signal-to-noise ratio. In the $\mathrm{ZnS}$ sample at the wavelength shown, only 32 shots were averaged for each point in the figure. In the highly transparent glasses to be discussed later, it was not unusual to average many hundreds or even thousands of shots.

Figure 5-8 shows the 2PA spectrum that was measured by thermal lensing in cvd $\mathrm{ZnS}$. The values agree better with the predictions for linearly polarized light than with predictions for randomly polarized light. As Figure 5-8 shows, the thermal lensing measurements generally follow both our pseudopotential predictions of Chapter 3 and Bae's experimental results, although the latter are for wurtzite, not polycrystalline zinc blende. The agreement between our thermal lensing values and the other values of $\beta$ is best near the absorption edge, where coincidentally the pump laser intensity was weakest.

Figure 5-8 was obtained by measuring $\beta$ at many frequencies-i.e., by forming many parabolic curves like gure 5-7-and then averaging those results in steps of $0.05 \mathrm{eV}$ along the horizontal axis. The step size is shown in Figure 5-8 by the horizontal error bar near $4.0 \mathrm{eV}$. Within a given horizontal window the average 


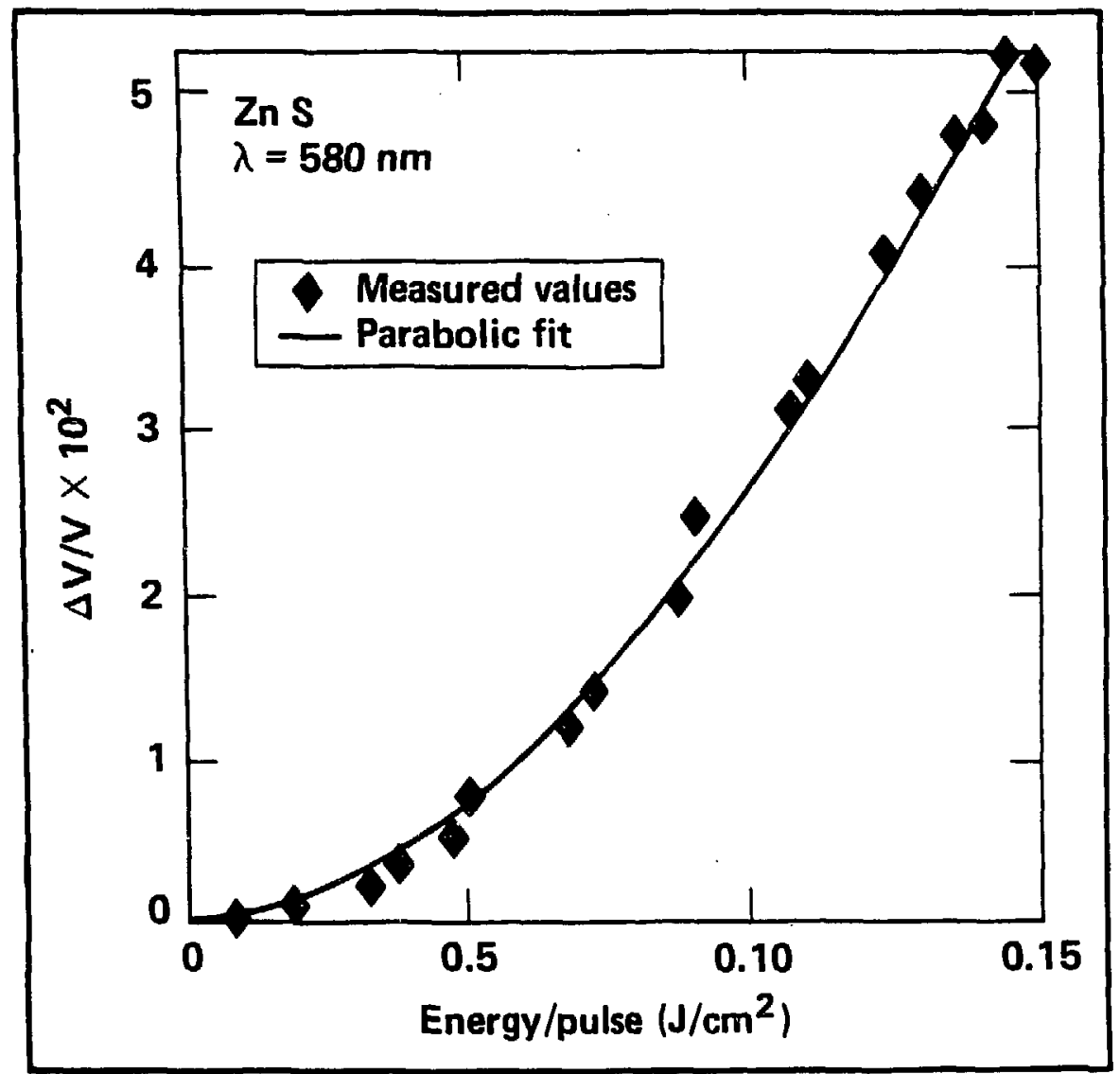

Figure 5-7. Thermal Lensing Signal in $\mathrm{ZnS}$ at $580 \mathrm{~nm}$. 


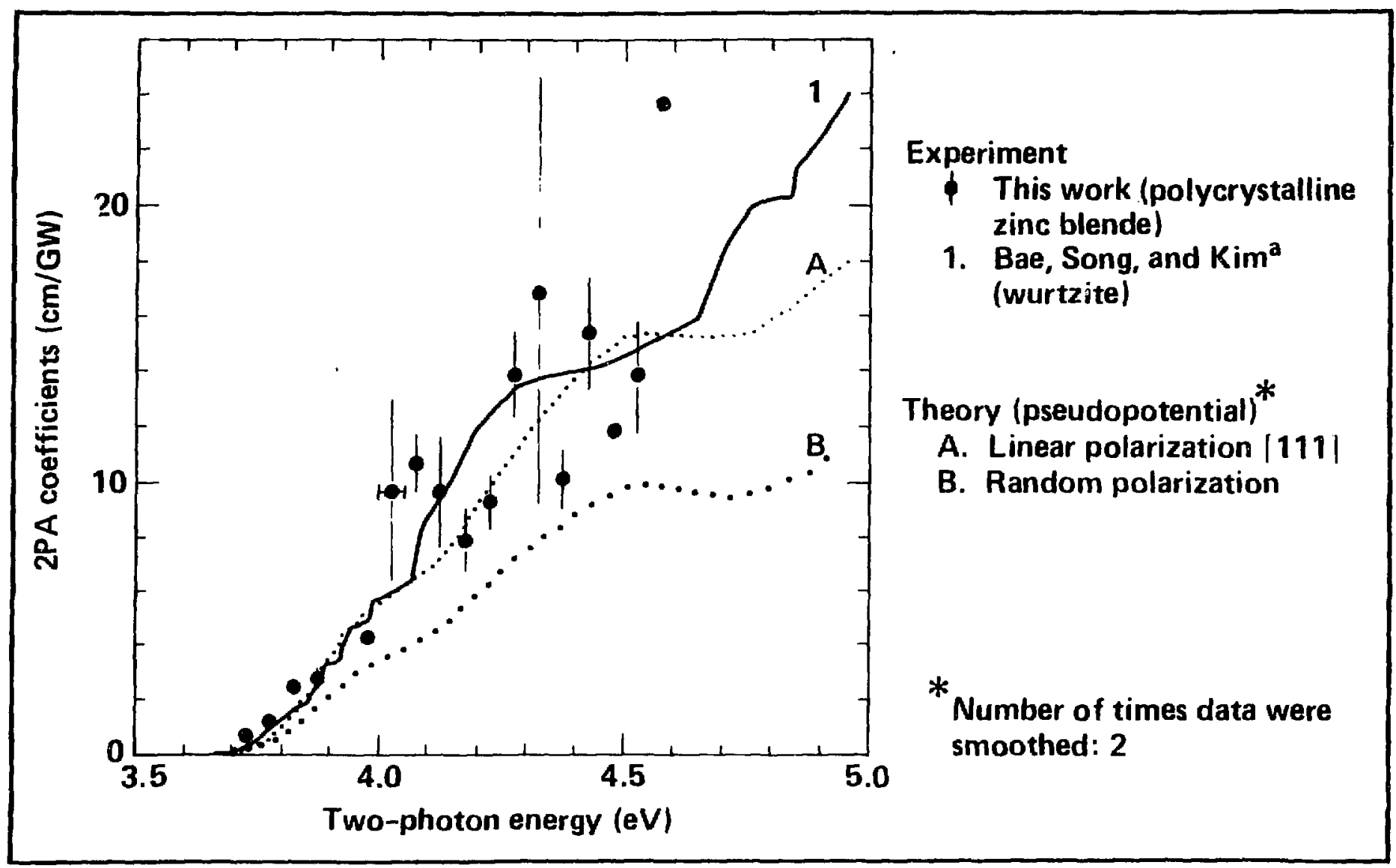

Figure 5-8. Two-photon Absorption in ZnS.

"llef. 2!. 
value and the rms vertical deviation were plotted. If a spectral window contained only one measured value, no vertical error bars were drawn. The error bars are smaller than the dots for the data nearest the absorption edge in Figure 5-8.

From the scatter in Figure 5-8, we estimate that our measurements are reproducible within $\pm 15 \%$. This is generally comparable to or greater than the size of the vertical error bars shown in the figure. The fact that the error bars are proportionately much greater for large absorption coefficients than for small suggests that nonlinear processes other than two-photon absorption may be occurring. This could include self-focusing or two-photon excited-state absorption. Note from Section 5.1.1 that the shot-to-shot fluctuations in the pump laser $( \pm 27 \%)$ were greater than the scatter in the spectrally averaged data shown in the figure. Thus, either pump beam variability or spurious nonlinearities might account for the scatter. The actual source of scatter was not conclusively isolated.

An unexpected source of noise in some of the $\mathrm{ZnS}$ data was microphonic interference. The experimental set-up was sufficiently sensitive to acoustic noise that an intercom speaker in the ceiling 5 feet above the sample had to be disconnected. By whistling, we determined that the strongest acoustical response of the sample occurred near $256 \mathrm{~Hz}$ (middle $\mathrm{C}$ ) and had a relative bandwidth of about $\pm 12 \%$ (a major second). Such microphonic behavior was not observed in other materials in this study, only in $\mathrm{ZnS}$. This suggests the utility of crd $\mathrm{ZnS}$ as photoacoustic transducer material.

The scatter reported in this work is much greater than that reported by others. Park and Waff and Bae et al. reported uncertainties of about 3\%. Panizza did not report any uncertainty. Therefore, in comparison to some dual-beam techniques and to single-beam photoacoustic measurements, photothermal lensing as performed in this work is less precise. Importantly, though, the measurement is calibrated, in 
contrast to the original photoacoustic work of Bae et al. As performed here, the measurements in $\mathrm{ZnS}$ were self-sufficient and used no reference sample. Precision might have been improved, according to the dicussion in Chapter 4, if a reference sample had been used. We defer further consideration of noise and accuracy until Section 5.6.

The fact that the thermal lensing results agree as well as they do with previous measurements over the range $3.6-4.0 \mathrm{eV}$ indicates that the technique is useful for roughly determining the magnitude of two-photon absorption in solids. An uncertainty of only $\pm 15 \%$ is excellent for almost all nonlinear optical measurements. Also, if one discards the three least precise measurements of Figure 5-8, then the experimental results follow the pseudopotential predictions to within the stated uncertainty of the measurements. This shows that the theory and measurements were consistent, although they were totally independent of one another.

\subsection{Results for $\mathrm{TiO}_{2}$ (Rutile).}

Syrthetic rutile is a transparent tetragonal crystal with an energy band gap around $3.1 \mathrm{eV}^{8}$ Waff and Park $^{9}$ and others ${ }^{10,11}$ have previously measured its 2PA coefficient, and so $\mathrm{TiO}_{2}$, like $\mathrm{ZnS}$, is a useful material for validating the thermal lensing technique for nonlinear spectroscopy of solids. Also, $\partial n / \partial \mathrm{T}$ is large in $\mathrm{TiO}_{2}$, which enhances the thermal lensing signal. Lastly, $\mathrm{TiO}_{2}$, like the laser glasses to be discussed in the next section, is an oxide. It is, therefore, chemically similar to the laser glasses in some respects. In particular, the highest occupied states are 2p-orbitals of the anion, oxygen, and the lowest empty states are orbitals of the cation, titanium. As in oxide glasses, optical absorption near the absorption edge is due to charge transfer of electrons from the anion to the cation. Table 5-2 lists values of parameters used to obtain the thormal lensing results in this work.

Because of its high melting point, $1840 \mathrm{C}, \mathrm{TiO}_{2}$ often contains chemical and 
Structure $^{a} \quad D_{4 h}^{14}(\mathrm{P} 4 / \mathrm{mnm})$

Density $(\mathrm{g} / \mathrm{cc})^{a} \quad \rho=4.25 \pm 0.03$

Specific Heat at $296 \mathrm{~K}(\mathrm{j} / \mathrm{cc} / \mathrm{K})^{a} \quad C_{p}=2.9$

Thermal Coefficient of Linear Expansion $\left(10^{-6} K^{-1}\right)^{a}$

$$
\alpha_{a}=8 \quad \alpha_{c}=9.2
$$

Refractive index at $0.6328 \mu \mathrm{m}^{a} \quad n_{o}=2.59 n_{e}=2.87$

Thermo-optic coefficient at $0.6328 \mu \mathrm{m}\left(10^{-6} K^{-1}\right)^{b}$

$$
\partial n_{o} / \partial T=81
$$

Table 5-2. Physical Properties of Rutile

${ }^{a}$ Ref. 12

"Estimated from Ref. 13 
structural defects. Common defects include displaced ions, occasional polytypism, and departure from stoichiometry. ${ }^{14}$ Manufacturing $\mathrm{TiO}_{2}$ in a reducing atmosphere favors the formation of defects and results in a black crystal due to a substoichiometry of oxygen. Some crystal growers dissolve small amounts (one part in $10^{6}$ ) of alumina in the titania in order to neutralize the color centers.

The sample in these experiments was a $15-\mathrm{mm}$ diameter by $1-\mathrm{mm}$ thick polished disk. Commercial Crystal Labs, Inc. supplied the boule from which the sample was cut. The sample did contain an undetermined small amount of aluminum, according to the manufacturer. A broad-band antireflection coating $\left(\mathrm{Al}_{2} \mathrm{O}_{3}\right)$ was applied to both faces of the sample to maximize transmission over the spectral range $0.6-0.7 \mu \mathrm{m}$. The sample was mounted in a miniature double goniometer (Charles Supper, Inc.) that was affixed to a small rotating stage (NRC). In this way 3-axis calibrated rotations were obtained so that the sample could be oriented with respect to the polarizations of the pump and probe beams and the direction of propagation. Laue X-ray analysis provided the orientation of the crystallographic axes with respect to the mount. Due to an error in slicing the sample from the boule, the optical axis of the crystal was approximately $8^{\circ}$ from normal to the polished faces of the sample. Twisting the sample prior to the thermal lensing measurement compensated for this.

The sample was aligned optically by placing it between crossed polarizers. It was twisted until a symmetric pattern of light and dark bands was transmitted through the rear polarizer. The resulting isogyre pattern was a cross centered on a set of concentric circles. To generate the high-angle scatter necessary for obtaining a large banded pattern, a folded piece of translucent tape was placed in the HeNe laser beam immediately in front of, but not touching, the sample. After the sample was oriented, the tape and crossed polarizers were removed. By placing the sample 
between crossed polarizers and translating it across the probe beam, we observed changes in the isogyre pattern. Therefore, the sample was either inhomogeneous, wedged, or both. The Laue X-ray tests implied that the $\mathrm{TiO}_{2}$ was nominally a single crystal.

The thermal lensing pump and probe beams propagated coaxially through the sample, approximately along the tetragonally symmetric z-axis. The two beams were linearly polarized nominally along orthogonal crystal axes. As discussed in Section 5.1, a Wollaston prism separated the pump and the probe beams behind the sample. Although such a prism contains optical cement, which may absorb in the presence of intense laser beams, absorption in the prism, if present, did not contribute to the thermal lensing signal.

Using a technique simiar to that described for $\mathrm{ZnS}$, we measured two-photon absorption in $\mathrm{TiO}_{2}$ over the range 0.58-0.75 $\mu \mathrm{m}(4.2 \mathrm{eV}>2 \hbar \omega>3.3 \mathrm{eV})$. As in Section 5.2, the thermal lensing signal at each wavelength was fit in a least squares sense to a quadratic function of pump beam fluence. The quadratic coefficient then provided the two-photon absorption coefficient. A rough spectrum was formed and then averaged over $0.05 \mathrm{eV}$ windows. Figure 5-9 shows the resulting visually averaged spectrum: A broad peak occurs near $2 \hbar \omega=3.6 \mathrm{eV}$, a wide relative minimum occurs near $2 \hbar \omega=3.8 \mathrm{eV}$, and above this, the absorption rises. The shape and magnitude of our spectrum agree with results of Waff and Park. In particular, the portions of their spectra that lie above $3.8 \mathrm{eV}$ agree with ours to within about $\pm 20 \%$. This agreement is a little worse than the $\pm 15 \%$ result presented for $\mathrm{ZnS}$ in the previous section.

Waff and Park conjecture that the peak near $3.6 \mathrm{eV}$ is excitonic and about $0.2 \mathrm{eV}$ below the true optical absorption edge. A key assumption behind this conjecture is that the true band-gap energy of $\mathrm{TiO}_{2}$ is $3.8 \mathrm{eV}$. However, elec- 


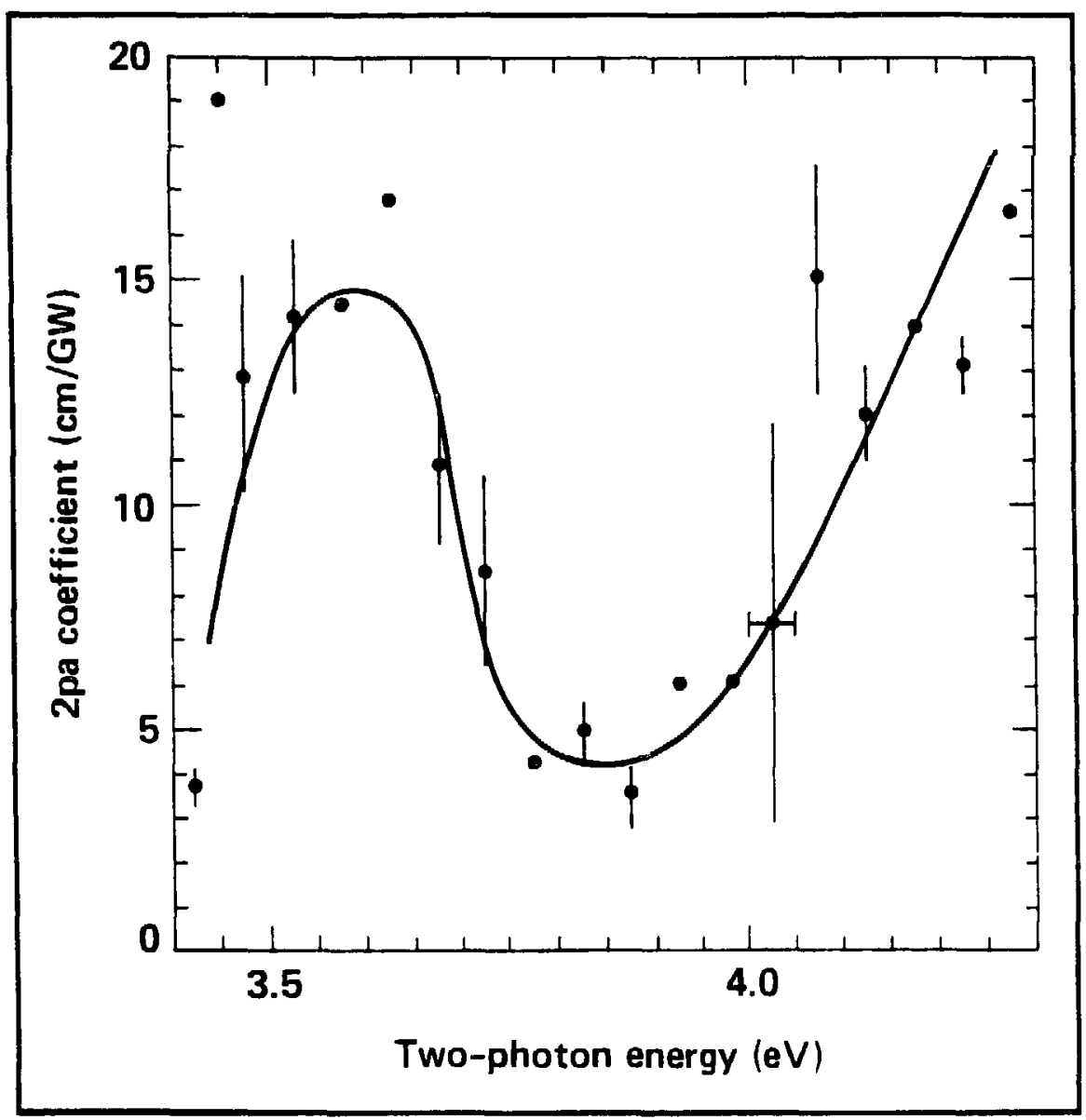

Figure 5-9. Two-Photon Absorption in $\mathrm{TjO}_{2}$ (Rutile). 
trochemical considerations, ${ }^{15}$ contact potential studies, ${ }^{16}$ and photoconductivity measurements ${ }^{17,18}$ all suggest that the absorption edge of Rutile is around $3.0 \mathrm{eV}$ rather than $3.8 \mathrm{eV}$. Thus, Waff and Park's interpretation of the significance of the peak is at odds with an abundance of independent determinations of the band-gap energy of rutile.

We propose an alternative explanation for the peak. It may be due to intrinsic deep impurity states. Centered $0.6 \mathrm{eV}$ above the indirect band edge at $3.0 \mathrm{eV}$ is a pair of split states due to trapped $\mathrm{Ti}^{3+}$, according to measurements by Ghosh. ${ }^{8}$ From the assignments by Ghosh et al., these states are split by $\pm 0.09 \mathrm{eV}$. Electroreflectance measurements show that a pair of weak lines occurs at $3.6 \pm 0.15 \mathrm{eV}$, possibly corresponding to transitions to these states. Importantly, the electroreflectance data do not show a strong dependence upon optical polarization.

Waff and Park found that the peak at $3.6 \mathrm{eV}$ vanished for light polarized perpendicularly to the tetragonal z-axis and was a maximum for light polarized along the z-axis. However, we detected a peak even though our beam was polarized normally to the z-axis. Our peak was about 2.5 times stronger than Waff and Park's peak for z-polarized light.

The reason for the disparity between their work and ours is not known. Some contributing factors could be temperature, impurities, and alignment. Waff and Park's experiments were at $77 \mathrm{~K}$, but ours were at room temperature. The samples were different and could have had differing quantities of chemical or structural defects. Also, our sample was possibly inhomogeneous, as mentioned earlier. This would have affected our ability to position the crystal axes absolutely with respect to the probe beam. This issue deserves future attention.

Despite the disparity between the data of Waff and Park and our data, certain common features should be emphasized. The location of the spectral peak at $3.6 \mathrm{eV}$ 
does not shift significantly with temperature. (This agrees with Waff and Park's findings.) The fact that both our results and Waff and Park's have a peak at $3.6 \mathrm{eV}$ shows that the absorption must be two-photon, not one-photon, since Waff and Park used a dual-beam technique, with wavelengths different than we used. Finally, as mentioned before, our data agree within 20\% of Waff and Park's results above $3.8 \mathrm{eV}$. This last fact shows again that the thermal lensing technique can provide accurate results, even without separate reference samples.

\subsection{Results in Oxide Glasses.}

Silicates, borates and phosphates are conventionally the three most important classes of oxide glass. Liu et al. and Smith previously measured absolute two-photon absorption at ultraviolet frequencies in fused silica ${ }^{19}$ and borosilicate glasses ${ }^{20-21}$ respectively, but no one has previously measured absolute two-photon absorption in compound silicates or phosphates. The ultraviolet frequencies that Smith used were sufficiently high that linear absorption probably contributed to two-photon absorption via two-step processes. ${ }^{20}$ According to Smith, at these frequencies the energy of two photons was several $\mathrm{eV}$ above the linear absorption edge of his borosilicate glasses, ${ }^{21}$ but it was below the absorption edge of his $\mathrm{SiO}_{2}$ sample. ${ }^{19}$ In reality, the energy of two photons may not have been above the true absorption edge of the borosilicate glasses. Typical absorption edges in borosilicates are greater than $5 \mathrm{eV},{ }^{23}$ but Smith cited values as low as $3.7 \mathrm{eV}$ for the absorption edge. ${ }^{21} \mathrm{~A}$ more correct statement is that the energy of two photons was above the "pseudo edge" of the glasses in those experiments. By "pseudo edge," we mean the photor - rgy at which linear absorption reaches $1.0 \mathrm{~cm}^{-1}$.

We measured the nonlinear absorption of several silicates (LG650, LG660, LG670, and LSG91H), borosilcates (BK3, BK7, and BK10), and a phosphate (LG750), looking for a correlation between the pseudo edge and the 2PA coefficient in oxide 
glasses. We fixed the optical wavelength at $0.5525 \mu \mathrm{m}(2 \hbar \omega=4.49 \mathrm{eV})$ and chose materials whose pseudo edges ranged from $3.5-4.5 \mathrm{eV}$. We chose a wavelength of $0.5525 \mu \mathrm{m}$, because the laser dye (R6G) worked well there and because that wavelength lay between absorption bands of $\mathrm{Nd}^{3+}$, a common impurity in laser glass. Further, $0.5525 \mu \mathrm{m}$ is between absorption bands of the ferric ion, Fe(III). Figure 510 shows the linear absorption spectra near the pseudo edges of our samples. From the figure, it is clear that the samples did not contain a large fraction of impurities.

Other than among the borosilicate samples there was no obvious correlation between the frequency of the pseudo edge and the magnitude of the nonlinear $a b-$ sorption coefficient that was measured. In the borosilicates, two-photon absorption was much stronger in $\mathrm{BK} 7$ than in $\mathrm{BK} 3$ or $\mathrm{BK} 10$, corresponding to the longerwavelength absorption edge of $\mathrm{BK} 7$, as shown in Figure 5-10. In $\mathrm{B}:=\mathrm{es}$, the magnitude of the norlinear absorption coefficient was of the order a few $\mathrm{cm} / \mathrm{TW}$ or less. This is about three to four orders of magnitude less than the two-photon absorption coefficients in $\mathrm{ZnS}$ or $\mathrm{TiO}_{2}$ and about one to two orders of magnitude less than the two-photon absorption coefficients that Smith measured in the ultraviolet for borosilicates. ${ }^{20-22}$ Table 5-3 shows our results.

As shown, all of the glass samples had a weak linear absorption. At higher fluences, the absorption increased nonlinearly. This suggests that the nonlinear absorption that we detected may have been two-step linear absorption. Our thermal lensing technique did not differentiate between two-photon absorption and two-step absorption. Figures 5-11 and 5-12 show the thermal lensing signals that we observed in our glasses. From these figures, one can plainly see that the slope of the signal approaches a non-zero constant as the fluence approaches zero, which indicates the 


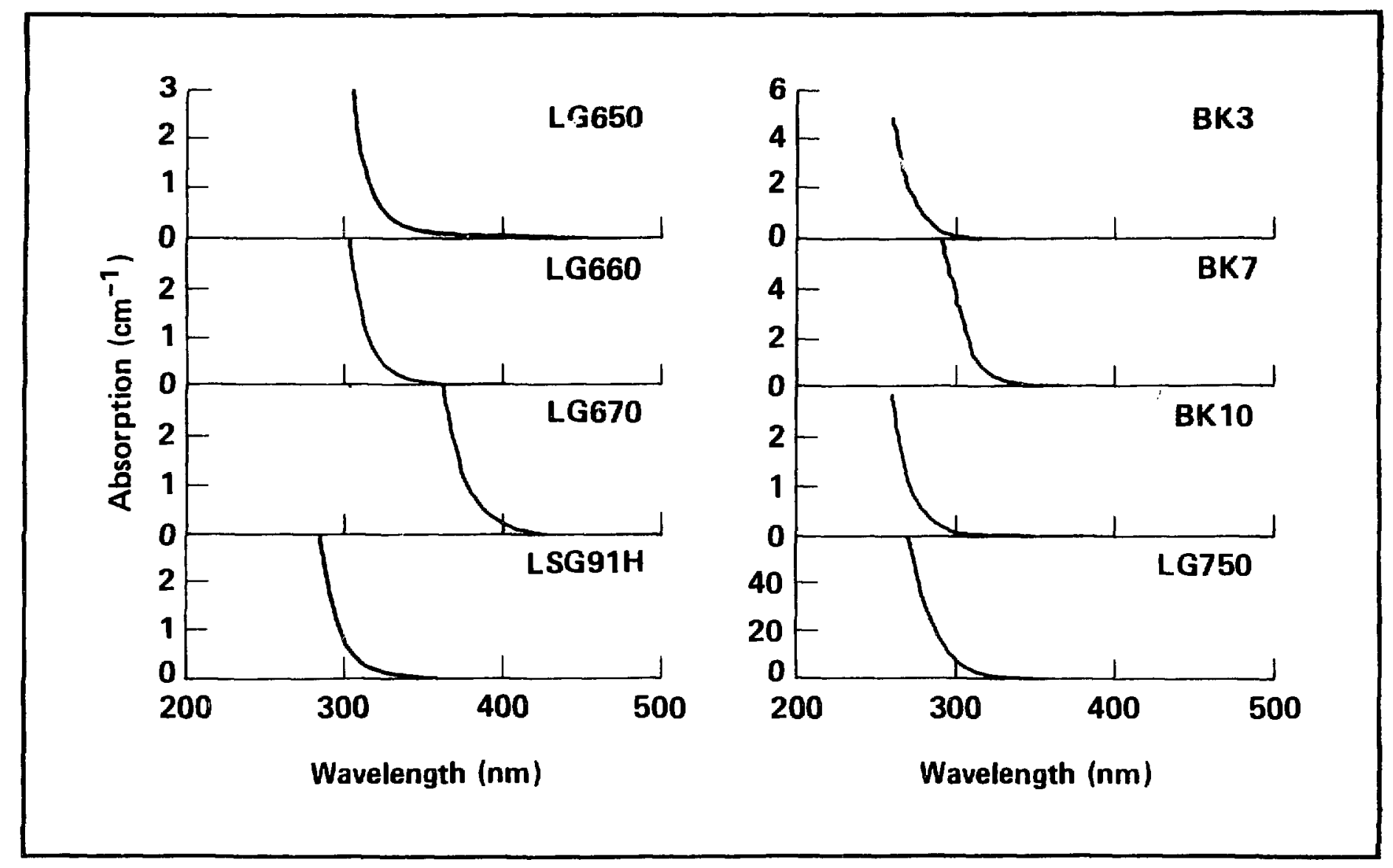

Figure 5-10. Linear Optical A bsorption Near the Band-Edge in Several Glasses. 
$-164-$

\begin{tabular}{|c|c|c|c|c|c|}
\hline \multirow[b]{3}{*}{ Glass } & \multirow{2}{*}{\multicolumn{2}{|c|}{$\begin{array}{l}\text { This Work } \\
0.5525 \mu \mathrm{m}\end{array}$}} & \multirow[b]{3}{*}{$\begin{array}{l}\text { Pseudo-edge } \\
(\mu \mathrm{m})\end{array}$} & \multicolumn{2}{|c|}{ Other Work } \\
\hline & & & & \multirow{2}{*}{$\begin{array}{c}0.532 \mu \mathrm{m} \\
\beta^{\mathrm{a}} \\
(\mathrm{cm} / \mathrm{TW})\end{array}$} & \multirow{2}{*}{$\begin{array}{c}0.351 \mu \mathrm{m} \\
\beta^{\mathrm{h}} \\
(\mathrm{cm} / \mathrm{TW})\end{array}$} \\
\hline & $\left(10^{-3} \mathrm{~cm}^{-1}\right)$ & $\begin{array}{c}\beta \\
(\mathrm{czu} / \mathrm{TW})\end{array}$ & & & \\
\hline \multicolumn{6}{|l|}{ Silicates } \\
\hline LG650 & 1.2 & $2.0 \pm 1.0$ & 0.318 & & \\
\hline LG660 & 0.4 & $<1.0$ & 0.317 & & \\
\hline LG670 & 1.1 & $0.4 \pm 0.4$ & 0.376 & & \\
\hline LSG91H & 0.7 & 0.0 & 0.296 & & \\
\hline $\mathrm{SiO}_{2}$ & 0.0 & 0.0 & $\left(E_{c ;}=7.9 e V\right)^{b}$ & & \\
\hline \multicolumn{6}{|c|}{ Borosiljcates } \\
\hline BK3 & 0.3 & $\leq 0.4$ & 0.280 & $0.6 \pm 0.6$ & 5.5 \\
\hline BK7 & 0.6 & $2 . \overline{7} \pm 1.5$ & 0.312 & $2.9 \pm 1.5$ & 30 \\
\hline BK10 & 0.6 & $\leq 0.5$ & 0.275 & $0.4 \pm 0.6$ & 15 \\
\hline \multicolumn{6}{|c|}{ Phosphates } \\
\hline LG750 & 0.0 & $<0.4$ & 0.320 & & \\
\hline
\end{tabular}

Table 5-3. Two-Photon Absorption Coefficients in Several Oxide Glasses.

${ }^{a}$ Ref. 3

'Ref. 21 
presence of linear absorption. Also, the deviation from linearity is usually small indicating that the nonlinear absorption is weaker than the linear absorption at the intensities we used.

We next attempted to obtain two-photon absorption spectra in the glasses, starting with BK7. BK7 had the largest nonlinear absorption of our glass samples. We determined relative values for $\beta$ at selected wavelengths from $555 \mathrm{~nm}$ to $575 \mathrm{~nm}$.

Figure 5-13 shows that $\beta$ does not change much over this spectral region, although at twice the photon energy $(275-290 \mathrm{~nm})$ the linear absorption coefficient rises sharply. Separate measurements ${ }^{3}$ at $0.532 \mu \mathrm{m}$ showed that $\beta$ in BK7 is essentially unchanged from its value at $0.5525 \mu \mathrm{m}$. Thus $\beta$ is nearly constant from $0.532 \mu \mathrm{m}$ to $0.575 \mu \mathrm{m}$ in BK7.

Beyond $575 \mathrm{~nm}$, the quantum efficiency of the dye laser limited the maximum pump intensity to values that were too low for us to detect two-photon absorption. We considered focusing the beam in order to raise the intensity, but we ruled this out because of problems with interpreting the results. Our spot size in the sample was $100 \mu$. Further reduction would have noticeably violated the thin lens approximation. Also, experience with measuring laser damage has shown that inhomogeneities in the glass become important at small beam diameters.

The nearly constant values of $\beta$ suggests that the specific transitions which lead to two-photon absorption in this spectral region are different from those which account for linear absorption at half the wavelength. Once again, the data scem to suggest that the nonlinear absorption that we have seen may likely be due to twostep absorption of impurities rather than to true two-photon absorption. Future work is warranted to resolve this issue. Due to the complex nature of glass, it is not possible to resolve it on the basis of our data. The most important conclusions that we can draw from our data are that nonlinear absorption does occur at these 

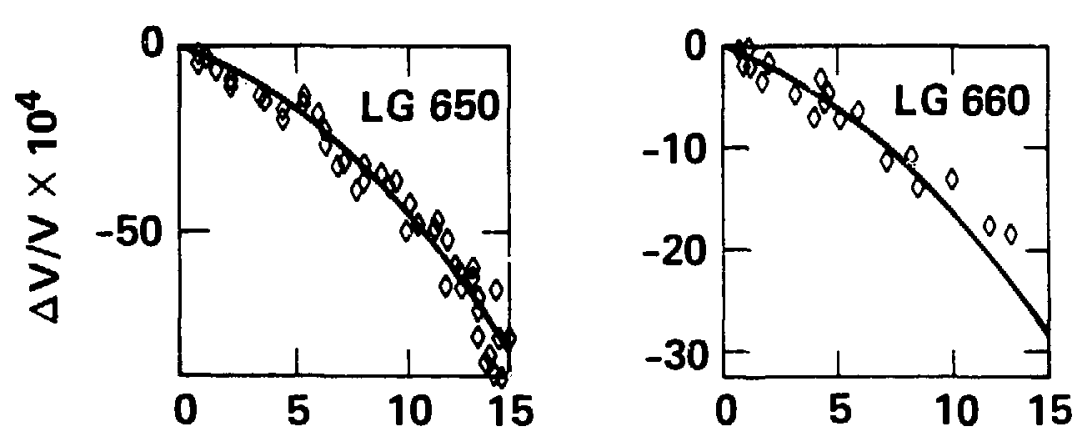

Fluence $\left(\mathrm{J} / \mathrm{cm}^{2}\right)$
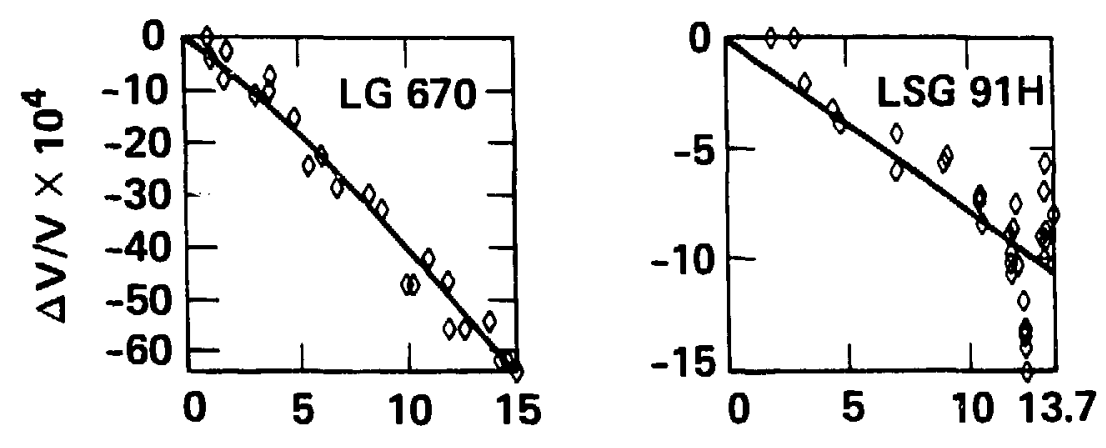

Fluence $\left(\mathrm{J} / \mathrm{cm}^{2}\right)$

Figure 5-11. Thermal Lensing Signal in Several Silicate Glasses. 

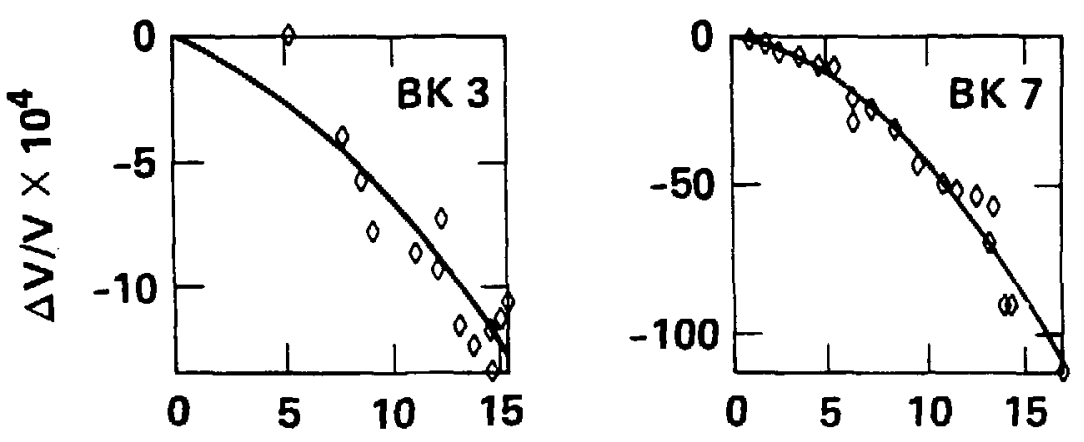

Fluence $\left(\mathrm{J} / \mathrm{cm}^{2}\right)$
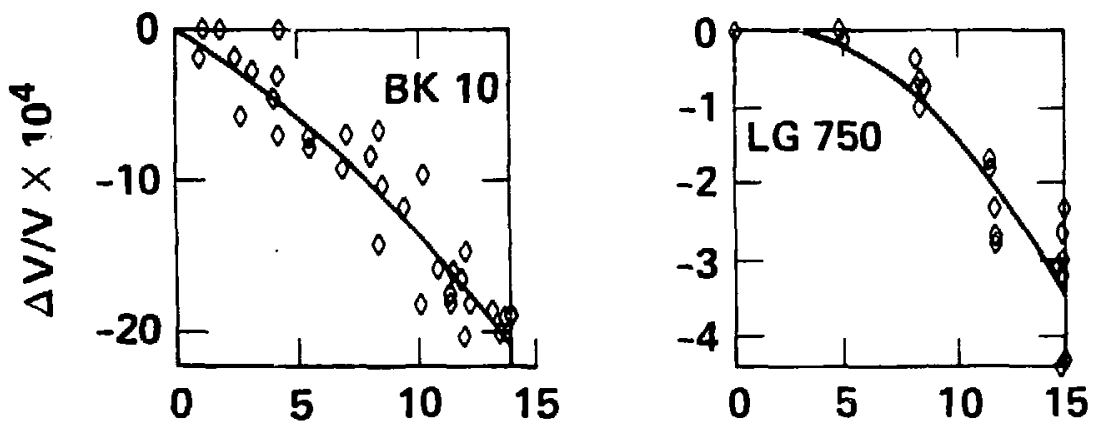

Fluence $\left(\mathrm{J} / \mathrm{cm}^{2}\right)$

Figure 5-12. Thermal Lensing Signal in Several Borosilicate Glasses $(B K 3, B K 7$, and $B K 10)$ and in One Phosphate Glass (LG750). 


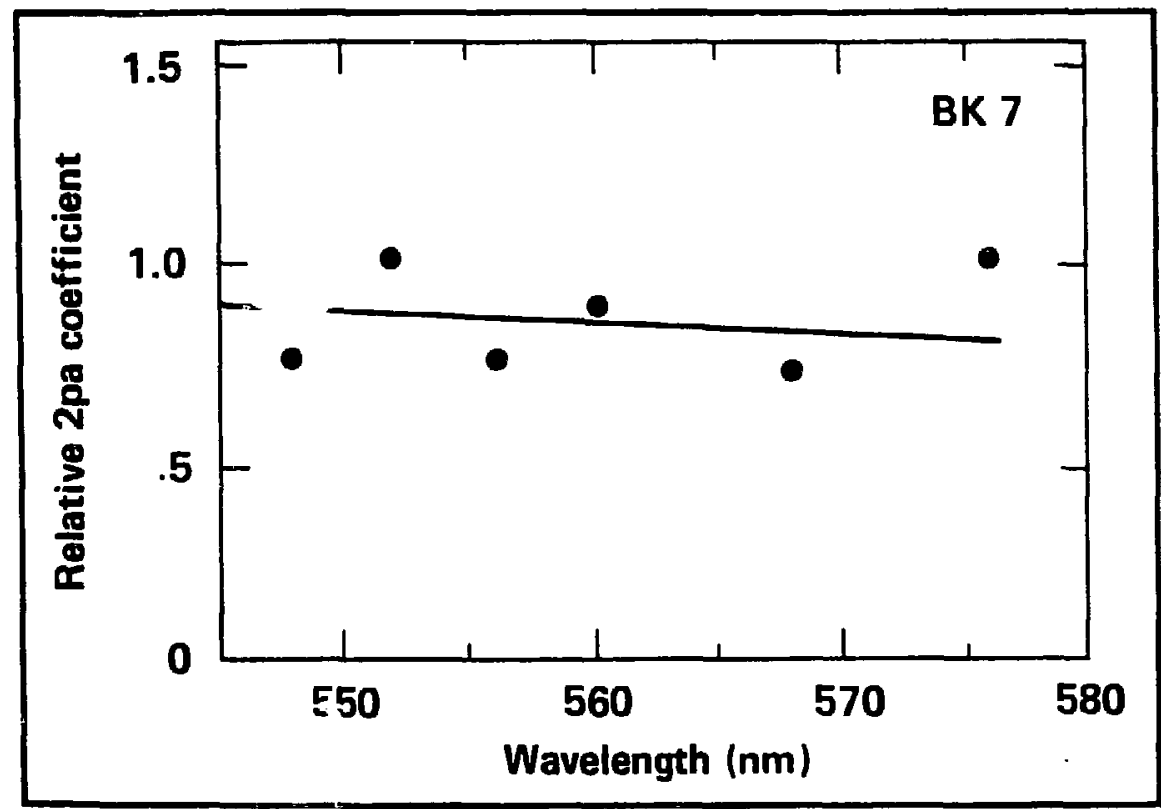

Figure 5-13. Two-Photon Absorption Coefficient in BK7 Measured by Thermal Lensing at Several Frequencjes. 
wavelengths and thermal lensing is sensitive enough to quantify it.

\subsubsection{Glass Compositions.}

In order to determine the composition of our samples we obtained chemical analyses from the Surface Science Section of the Chemistry and Materials Science Department at Lawrence Livermore National Laboratory. The exact chemical compositions of the glasses may not be published without violating the proprietary agreements between LLNL and the glass manufacturers. Table 5-4 shows the general compositions of the glasses.

To check for impurities in our samples, we used conventional linear absorption spectroscony supplemented with visual observations of flourescence. Absorption measurements from 0.2-1.2 $\mu \mathrm{m}$ showed no obvious spectral structure (See Fig. 5-10). Under $1: 35-\mu \mathrm{m}$ illumination, flourescence at visible frequencies was observable in only two samples, LG670 and LSG91H. The latter was a barely perceptible bluish white color whose physical origin was not determined, and the former was a bright blue that was attributable to the presence of $\mathrm{CeO}$. Under $0.25 \mu \mathrm{m}$ illumination, all samples fiuoresced slightly. The silicates were generally pale blue, the borosilicates were yellow, and the phosphate was pale aqua. It was not possible to identify the sources of fluorescence. In glasses, fluorescence is specirally broad, and the center wavelength of a fluorescer changes with valence and with ionic environment. The weakness of the fluorescence that was observed suggests, however, that whatever the sources of fluorescence were, the products of their oscillator strengths and their concentrations were low.

We did not find a clear correlation between composition and nonlinear absorption, which further suggests that impurities may have caused the nonlinear absorption. 


\begin{tabular}{|lccc|}
\hline Glass & Manufacturer & Network Former & Network Modifiers \\
\hline LG650 $^{a}$ & Schott & Silicate & Alkali \\
LG660 $^{b}$ & Schott & Silicate & Alkali, Zinc \\
LG670 $^{b}$ & Schott & Silicate & Lithium, Aluminum \\
LSG91H $^{c}$ & Hoya & Silicate & Alkali, Aluminum \\
BK3 $^{d}$ & Schott & Borosilicate & Alkali, Aluminum \\
BK7 $^{d, e}$ & Schott & Borosilicate & Alkali, Barium, Aluminum \\
BK10 $^{d}$ & Schott & Borosilicate & Alkali, Aluminum \\
LG750 $^{b}$ & Schott & Phosphate & Potassium, Barium, Aluminum \\
& & & \\
\hline
\end{tabular}

Table 5-4. Glass Types Tested

\footnotetext{
aManufacturer's Preliminary Data Sheet.

${ }^{b}$ Manufacturer's Data Sheet.

${ }^{c}$ Courtesy Hoya Optics, Inc.

${ }^{d}$ Courtesy Dick Ryon, Chemistry and Materials Science Division, LLNL.

'Ref. 24, Sample 6517.
} 


\subsubsection{Measurement Technique in Glasses.}

The energy calibration, relative fluence, and temporal pulse-shape of the pump beam were determined as described in Section 5.1. The samples were uncoated, the angle of incidence was nearly normal, and the pump and probe beams were linearly polarized in orthogonal directions. Each sample was nominally 1-cm thick. The thermo-optical properties were not known for all of the glasses, and so we calibrated our two-photon absorption measurements against one-photon absorption measurements in doped samples. The next section describes how this was done.

\subsection{Calibration of Thermal Lensing Measurements with Doped Samples.}

To calibrate our two-photon absorption measurements, we used doped, linearly absorbing samples of the same host composition as our undoped glasses. Conventional photometric measurements of linear absorption were made and were then compared with the thermal lensing measurements of the same samples. Using published values of thermo-optic coefficients and specific herts where available, the thermal lensing results were generally $30-50 \%$ higher than the spectrophotometric results. There are several possible factors responsible for this: departures from Gaussian beam profiles, errors in measuring the divergence or size of the laser beams at the sample, and the possibility that the effective thermo-optic coefficients $(\partial n / \partial T)$ were different from those reported by the glass manufacturers. Since the

spectrophotometer (Cary 17) was well calibrated and since the pump and probe beams were well characterized, the disparity between the thermal lensing and the conventional measurements was attributed to changes in the effective thermo-optic coefficients of the glasses.

\subsubsection{Effective Thermo-Optic Coefficients.}

The thermo-optic coefficient of a solid may be either positive or negative. The sign depends on whether or not small chariges of temperature raise the microscopic 
polarizability faster than they lower the density. In the glass samples that were discussed in the previous section, the values of the thermo-optic coefficients as measured by thermal lensing were, with one exception (LSG91H), higher than the values cited by the manufacturers.

In the simple theory of Chapter $4, \partial n / \partial T$ was presumed to be measured under conditions of constant stress or pressure, which is the usual condition. The thermal stress in a thermal lens is not spatially uniform, however. This is due to the constrained thermal expansion caused by the resistance of the bulk of the surrounding sample to changes in the size of the lens. The clamped expansion prevents the density of the thermal lens from dropping, as it would under free expansion. This in turn leads to more-positive values of $\partial \mathrm{n} / \partial \mathrm{T}$ than are typically cited in the literature.

Our doped samples contained $0.2-2.0$ weight-percent $\mathrm{Nd}_{2} \mathrm{O}_{3}$, depending on the sample. At these concentrations, the inter-ion cross relaxation is believed neglibible. ${ }^{3}$ With a Cary 17 spectrophotometer we measured the linear absorption spectra of the samples, correcting each measurement for losses due to surface reflections and scatter. Figure 5-14 shows a typical spectrum obtained from the Cary 17. The baseline is the dashed line. After measuring the linear absorbance photometrically, we measured the thermal lensing of the same samples, tuning the dye laser to the $\mathrm{Nd}$ absorption peak near $0.571 \mu \mathrm{m}$. The thermal lensing signal varied linearly with fluence of the dye laser beam. Using Eq. (5.6), we determined the product of the linear absorption coefficient, $\alpha$, times $(1-\eta) \ell^{-1} \partial(n \ell-\ell) / o v e r \partial T$.

From the spectrophotometric values of $\alpha$, we then found the $\frac{(1-\eta)}{C \ell \theta(n \ell-\ell) / o v e r a T}$. In this set of terms, the specific heat, $C$, was taken as the specific heat per unit volume at constant pressure since the ratio $C_{p} / C_{v}$ is approximately unity in solids. Then we measured the effective thermal conversion efficiency $(1-\eta)$, which gave 


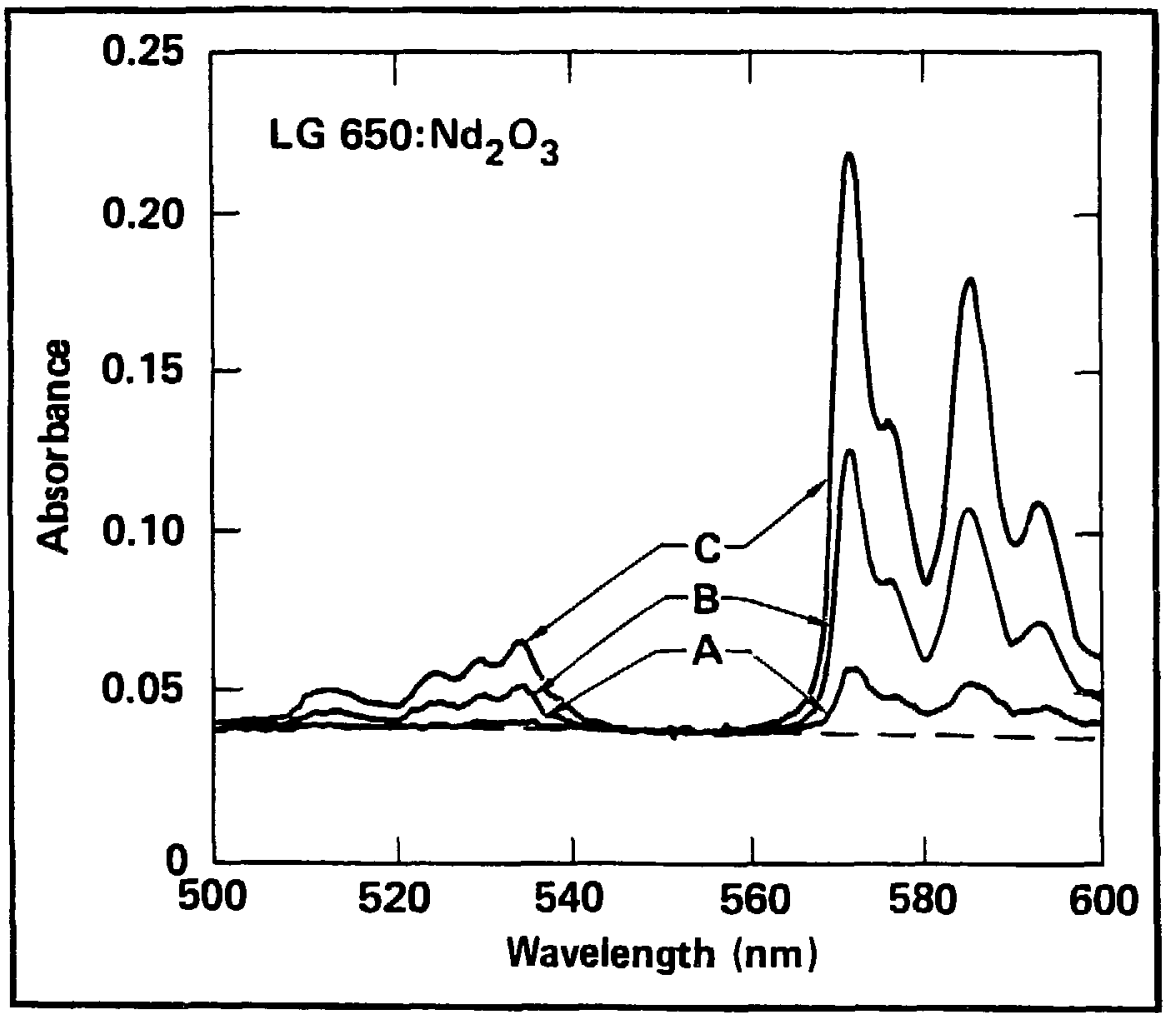

Figure 5-14. Typical Linear Absorption Spectrum of $\mathrm{Nd}^{3+}$ in an Optical Glass. The glass shown is LG650, a silicate. Curves $A, B$, and $\mathrm{C}$ represent increasing concentrations of $\mathrm{Nd}^{3+}$. The background signal is shown as a dashed line. 


\begin{tabular}{|c|c|c|c|c|}
\hline $\begin{array}{l}\left.\frac{1}{C} \frac{1}{\ell} \frac{\partial(n \ell-\ell)}{\partial T}\right|_{\text {meanured }} \\
\left(10^{-6} \mathrm{~cm}^{3} / \mathrm{J}\right)\end{array}$ & $\begin{array}{c}C_{p} \\
\left(\mathrm{~J} / \mathrm{cm}^{3} / \mathrm{K}\right)\end{array}$ & $\begin{array}{c}\frac{1}{\ell} \frac{\partial \ell}{\partial T} \\
\left(10^{-6} K^{-1}\right)\end{array}$ & $\begin{array}{c}\left(\frac{\partial n}{\partial T}\right)_{e f f} \\
\left(10^{-6} K^{-1}\right)\end{array}$ & $\begin{array}{c}\left(\frac{\partial n}{\partial T}\right) \\
\left(10^{-6} K^{-1}\right)\end{array}$ \\
\hline LG650 3.8 & 2.0 & 9.5 & +2.7 & $-1.9^{1 a .3}$ \\
\hline LG660 4.3 & 1.9 & 10.7 & +2.6 & $+1.1^{1 h .3}$ \\
\hline LG670 6.1 & 2.3 & 9.3 & +0.0 & +.9 \\
\hline LSG91H 3.1 & $1.8^{2}$ & 9.0 & +5.3 & +1.6 \\
\hline BK7 3.4 & $2.2^{1.4}$ & 7.1 & +3.8 & $+1.5^{1 d .3}$ \\
\hline LG750 1.7 & $2.0^{1 e}$ & 11.4 & -2.5 & -5.1 \\
\hline
\end{tabular}

\section{Table 5-5. Effective Thermo-optic Coefficients}

1. Ref. 24 .
a. \#6501
b. \#6618
c. \#5019
d. \#6517
e. \#6667

2. Hoya Optics, Inc., 3400 Edison Way, Fremont, Ca 94538, Mfr. data sheet.

3. Schott Optic Glass, Inc., Technologies, 400 York Ave., Duryea, PA, Mfr. data sheet. 
us a value for the thermo-optic term $\ell^{-1} \frac{\partial}{\partial T}(n \ell-\ell)$. Section 5.5 .2 discusses how we measured $\eta$. Table 5-5 compares the values of the thermo-optic coefficients that were measured by thermal lensing to manufacturer's values obtained under constant pressure.

\subsubsection{Determination of the Effective Thermal Conversion Efficiency $(1-\eta)$.}

Following the absorption of a visible photon, $\mathrm{Nd}^{3+}$ relaxes in three steps, as depicted in Figure 5-15. ${ }^{25}$ Rapidly after photoexcitation, the $\mathrm{Nd}^{3+}$ ion relaxes nonradiatively to the ${ }^{4} F_{3 / 2}$ state, the metastable upper level in the Nd:YAG laser. Next, within a few hundred microseconds the ion relaxes radiatively with a probability $W_{\text {rad }} / W_{\text {tot }}$ to one of four levels in a manifold of states labelled ${ }^{4} I_{J}(J=9 / 2,11 / 2$, $13 / 2$, or $15 / 2$ ). The rates $W_{\text {rad }}$ and $W_{\text {tot }}$ are the radiative decay rate per excited ion and the total decay rate per excited ion, respectively. Finally, the ion relaxes vibronically to the ground state. The total relaxation time is much shorter than the thermal diffusion time, which is a few milliseconds in our experiment.

The effective luminescent efficiency $\eta$ is the ratio of the average photon energy radiated to the average photon energy absorbed. If $\hbar \omega$ denotes the energy of an incident photon, and if $\rho_{J}$ is the radiative branching ratio of the transition ${ }^{4} \mathrm{~F}_{3 / 2}$ to ${ }^{4} I_{J}$, then $\eta$ is given by

$$
\eta=\left(W_{\text {rad }} / W_{t o t}\right) \sum_{J} \frac{\rho_{J} \hbar \omega_{J}}{2 \hbar \omega}
$$

where $\hbar \omega_{J}$ is the transition energy from ${ }^{4} F_{3 / 2}$ to ${ }^{4} I_{J}$. The radiative transition rate from ${ }^{4} \mathrm{~F}_{3 / 2}, W_{\text {rad }}$, is calculated from a Judd-Ofelt ${ }^{24,26}$ model in the limit of $0 \mathrm{~K}$ and low $\mathrm{Nd}$ concentration. The branching ratios $\rho_{J}$ are determined from the relative heights of the absorption peaks in the Cary 17 spectrum of each sample. The total decay rate, from ${ }^{4} \mathrm{~F}_{3 / 2}, W_{t o t}$, is determined by measuring the fluorescent lifetime of the samples at room temperature. Typically, for $\hbar \omega \approx 2 \mathrm{eV}$, one has $1-\eta \approx 0.5$ for Nd-doped glasses. 


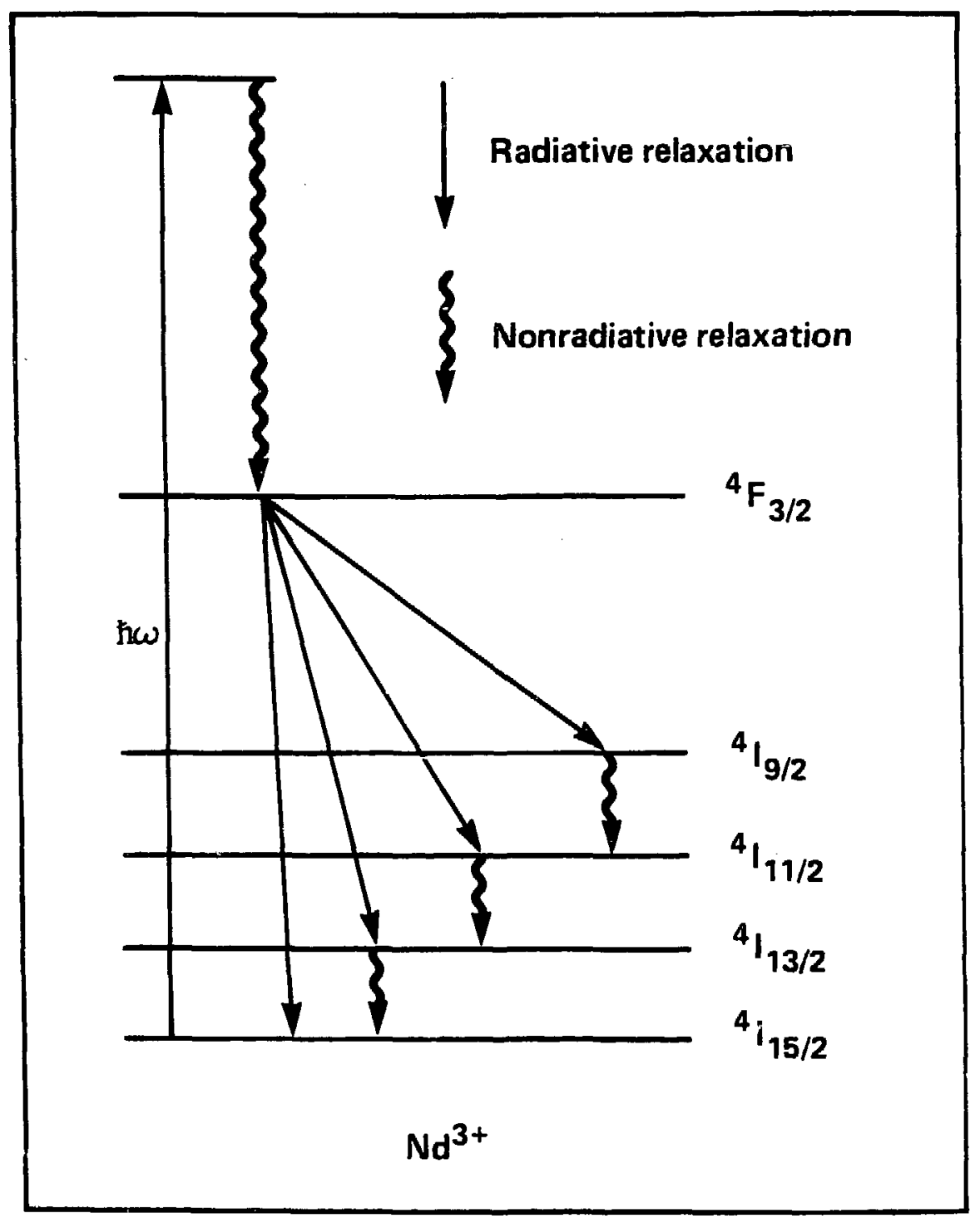

Figure 5-15. Thermal Relaxation of $\mathrm{Nd}^{3+}$ Ions in Glass. 


\subsection{Accuracy.}

In this section we enumerate the major sources of uncertainty in our measurements. They are (1) laser fluctuations, (2) material parameters, (3) intensity :alibration, and (4) electronic noise.

Fluctuations in the probe laser limited the precision with which we could measure the thermal lensing signal, $S_{0}=\Delta V / V$. At a frequency of $60 \mathrm{~Hz}$, the fluctuations had an rms peak-to-peak value of $0.3 \%$ of the dc intensity, as mentioned in Section 5.1. The third and the fifth harmonics of this noise had amplitudes on the order of 0.0001 times the dc intensity. This noise was comparable in amplitude and frequency content to the weakest thermal lensing signals that we detected. From Eq. (5.6), we can estimate the limitation that this imposed on linear absorption alone. Typical values of the parameters in Eq. (5.6) are $\lambda=0.6328 \times 10^{-4} \mathrm{~cm}$, $C=2 \mathrm{~J} / \mathrm{cm}^{2}, \ell^{-1} \partial(n \ell-\ell) / \partial T=10^{-6} \mathrm{cmK}, w_{1} / w_{p}=1$, and $B=1 / 2$. This gives a minimum detectable energy per area of

$$
\operatorname{Min}\left(\alpha \Gamma_{0} \ell\right)=0.005 \mathrm{~J} / \mathrm{cm}^{2} .
$$

Note that the minimum detectable quantity in Eq. (5.10) is an absorbed fluence, not an absorption coefficient. This simply reflects the fact that longer samples or hotter fluences give more signal. Since the maximum values of fluence that we used were on the order of $15 \mathrm{~J} / \mathrm{cm}^{2}$ and the sample thicknesses were about $1 \mathrm{~cm}, \mathrm{Eq}$. (5.10) shows that the minimum detectable absorption coefficient was predicted to be about $3 \times 10^{-4} \mathrm{~cm}^{-1}$ at a signal-to-noise ratio of unity. This predicted value was approximately our observed lower limit.

Both temporal and spatial fluctuations contributed to the overall scatter in the results. The former primarily affected the value of the pulse shape parameter, $h^{(2)}$, but the latter affected the size, shape, and position of the pump beam. Consequently, the latter affected the validity of the model that we used for analyzing 
the thermal lensing data, which assumed that the pump and probe beams were Gaussian functions of space, identical from shot to shot. Checking the value of $h^{(2)}$ from time to time during an experiment, we found that it could vary by as much as $20 \%$ during a long run. The scatter of the measurements in some glasses was as high as $\pm 50 \%$ suggesting that spatial fluctuations were at least as important as temporal fluctuations. We also considered the possibility that self-focusing might be present. For our intensities (less than $2 \mathrm{GW} / \mathrm{cm}^{2}$ ) and sample thicknesses (1-cm), we discounted it based on known values of the nonlinear refractive index coefficient $n_{2} \cdot 27,28$

Uncertainty in the material parameters affected the accuracy of the measurements. The three material parameters that were least certain were the effective radiative efficiency $\eta$, the effective thermo-optic coefficient $\partial n / \partial T$, and the effective coefficient of thermal expansion $\ell^{-1} \partial \ell / \partial T$. To analyze our data, we assumed that the radiative efficiency was zero in all of our transparent samples. This could be justified on the grounds that (a) in the crystals, our measurements agreed with previous data and (b) in the glasses, the presence of significant numbers of impurities or defects would have been evident in the linear absorption spectra. When we failed to obtain thermal lensing values for linear absorption that agreed with spectrophotometric measurements in doped, linear absorbers in the glasses we measured the effective value of $\partial(n \ell-\ell) / \partial T$. We attributed the difference between the thermal lensing and the spectrophotometry to variations in the thermo-optic parameters, as discussed in the previous section. It is possible that our assumptions were invalid, which would change the inferred values of $\alpha$ and $\beta$ by $10 \%-40 \%$.

Uncertainty in the calibration of the energy meters was estimated at $\pm 15 \%$, maximum. This was a conservative estimate, judging by the agreement between our measured values and those of other workers for $\beta$ in $\mathrm{ZnS}$ and 1 'iO $\mathrm{O}_{2}$ - especially 
in view of the uncertainties that we have already listed. The calibration did not affect the repeatability of the measurements.

Electronic noise was a neglible $2 \%$. This applies to all amplifiers, oscilloscopes, and signal averagers.

Compared to other techniques for measuring nonlinear absorption, we found our results to be generally less precise than dual-beam transmission measurements (e.g., Panizza and Park and $W_{+} f f, 3 \%$ ) or single-beam photoacoustic measurements (Bae, Song and Kim, 3\%). The chief reason for this, we believe, is the noise in our lasers. One subtle problem that we did not remedy was the fact that our pump laser was forced to operate at $10 \mathrm{~Hz}$, a subharmonic of $60 \mathrm{~Hz}$. This was due to the way the video system was synchronized. As a result of operating at $10 \mathrm{~Hz}$, we were almost phase-locked to the main electrical power and hence to the fluctuations in the probe beam intensity. (Phase locking was not complete, because a quartz oscillator controlled our timingr circuitry independently of any power line fluctuations.) With a different set of hardware, one might be able to reduce the noise floor by as much as an order of magnitude. This would enable one to measure weaker signals, but that may be unnecessary as far as measuring nonlinear absorption in solids is concerned. As Figs. 5-11 and 5-12 show, the factor that limited our results in glass was the smallness of $\beta$ relative to $\alpha$. That is, more intensity, not less noise, was generally required in order to distinguish linear and nonlinear absorption. (The minimum intensity to make two-photon absorption comparable to one-photon absorption is risughly $\alpha / \beta$.) Overall uncertainty of the measurements is about $\pm 20 \%$ for the crystals and $\pm 15 \%-100 \%$ for the glasses. The two-photon absorption coefficients in the glasses were about four orders of magnitude weaker thian in the crystals.

\subsection{Summary.}

This chapter has discussed photothermal lensing measurements of nonlinear 
absorption in solids. Thermal lensing was chosen because of its potentially high sensitivity (one part in $10^{6}$ in liquids) and its novelty. Prior to this work, no one had used pulsed thermal lensing to meaure nonlinear absorption in highly transparent solids. We found that, while we could not achieve as high a sensitivity as thermal lensing has demonstrated in liquids, we were able to detect absorption of a few parts in $10^{4}$. This was adequate to measure nonlinear absorption in optical glasses, provided that the intensity was of the order of $1 \mathrm{GW} / \mathrm{cm}^{2}$. The difference in the sensitivity of our measurements and the potential sensitivity that others have quoted was due to the lower thermo-optic coefficients $(\partial n / \partial T)$ of solids vis-à-vis liquids and to signal-to-noise limitations in our probe laser.

With $\mathrm{ZnS}$ and $\mathrm{TiO}_{2}$, we demonstrated that our method of thermal lensing could be a stand-alone technique with expected uncertainties of about $\pm 20 \%$ in measuring two-photon absorption in crystals with band-gap energies between 3 and 4 $\mathrm{eV}$. Results were less accurate for glasses. Thermal lensing results disagreed with spectrophotometric measurements of one-photon absorption in doped samples. We attributed this to uncertainties in the thermo-optic coefficient. We measured the thermo-optic coefficients of a set of transparent glasses by using Nd-doped reference samples. Then we tested the undoped glasses for nonlinear absorption. We found that all of the transparent samples exhibited a weak linear absorption that was on the order of $10^{-3} \mathrm{~cm}^{-1}$. At higher intensities, the absorption increased. The effective two-photon absorption coefficients of the glasses were of the order of $1 \mathrm{~cm} / \mathrm{TW}$. The presence of a weak linear absorption that was stronger than the nonlinear absorption suggested that the latter may have been due to excited-state, linear absorption. Our measurements could not determine whether the absorption was instantaneous. Additionally, we found no obvious correlation between the nonlinear absorption and any other intrinsic physical parameter. This suggested 
that the nonlinear absorption was related to impurities. although we attempted to minimize the effects of certain impurities like $\mathrm{Nd}^{3+}$ and $\mathrm{Fe}^{3+}$ by making our measurements at a wavelength where those impurities were non-absorbing. Further tests are required to determine to what degree the absorption was two-step versus truly two-photon and whether it was impurity-related. The strongest conclusion to be drawn from these measurements is that the thermal lensing technique used here was sufficiently sensitive to quantify the very weak nonlinear absorption in highly transparent glasses. 


\section{References}

1. H. Lotem and C. B. de Araujo, "Absolute determination of the two-photonabsorption coefficient relative to the inverse Raman cross section," Phys. Rev. B 18(4), 1711-16 (15 Aug 1977).

2. A. Yariv, Quantum Electronics, Second Edition (John Wiley and Sons, Inc., New York, 1975).

3. W. T. White, III, M. A. Henesian, and M. J. Weber, "Photothermal lensing measurements of two-photon absorption and two-photon-induced color centers in borosilicate glasses at $532 \mathrm{~nm}$," to be published in J. Opt. Soc. Am. B 2(9) (Sept. 1985).

4. P. W. Smith, M. A. Duguay, and E. P. Ippen, "Optical correlation technique for pulse shape measurement," in Progress in Quantum Electronics 3(2), J. H. Sanders and S. Stenholm, editors (Pergamon Press, Oxford, 1974).

5. W. B. Jackson, N. M. Amer, A. C. Boccara, and D. Fournier, "Photothermal deflection spectroscopy and detection," Appl. Opt. 20(8), 1333-44 (15 Apr. 1981).

6. R. W. G. Wyckoff, Crystal Structures, Volume 1 (Interscience, New York, 1963).

7. Gmelins Handbuch der Anorganische Chemie, Zink, System-Nummer 32 (Verlag Chemie, G. m. b. H., Weinheim, 1956).

8. A. K. Ghosh, F. G. Wakim, and R. R. Addiss, Jr., "Photoelectronic processes in rutile," Phys. Rev. 184(3), 979-88 (15 Aug 1969).

9. H. S. Waff and K. Park, "Structure in the two-photon absorption spectrum of $\mathrm{TiO}_{2}$ (rutile)," Phys. Lett 324(2), 109-10 (15 June 1970).

10. G. Kobbe and C. Klingshirn, "Quantitative investigation of the two-photon absorption of ruby-laser-light in various semicunductors," Z. Physik B 37(1), 9-12 (1980). 
11. G. A. Royce and R. B. Kay, "Multiphoton interactions in rutile," Appl. Opt. 23(12), 1975-9 (15 June 1984).

12. Gmelins Handbuch der Anorganische Chemie, Titan, System-Nummer 41 (Verlag Chemie, G. m. b. H., Weinheim, 1951).

13. T. Toyoda and $M$. Yabe, "The temperature dependence of the refractive indices of $\mathrm{SrTi0}_{3}$ and $\mathrm{TiO}_{2},{ }^{\prime} J$ J. Phys. D 16(12), L251-55 (Great Britain, $14 \mathrm{Dec} 1983$ ).

14. F. A. Grant, "Properties of rutile (titanium dioxide)," Rev. Mod. Phys. 31(3), 646-74 (July 1959).

15. D. E. Scaife, "Oxide semiconductors in photoelectrochemical conversion of solar energy," Solar Energy 25, 41-54 (Pergamon Press, Great Britain, 1980).

16. K. W. Frese, Jr., "Simple method for estimating energy levels of solids," J. Vac. Sci. Technol. 16(4), 1042-44 (Jul./Aug. 1979).

17. D. C. Cronemeyer, "Electrical and optical properties of rutile single crystäls," Phys. Rev. 87(5), 876-86 (1 Sept 1952).

18. A. Fujishima and K. Hondo, "Electrochemical photolysis of water at a semiconductor electrode," Nature 238, 37-38 (7 July 1972).

19. P. Liu, W. L. Smith, H. Lotem, J. H. Bechtel, N. Bloembergen, and F. Adhav, "Absolute two-photon absorption coefficients at $355 \mathrm{~nm}$ and $266 \mathrm{~nm}$, Shys. Rev. B 17(12), 4620-32 (15 June 1978).

20. W. L. Smith, C. L. Vercimak, and W. E. Warren, "Excited-state absorption cross-section in ultraviolet glasses following two-photon absorption," J. Opt. Soc. Am. 72(12), 1782 (Dec 1982).

21. W. L. Smith, in Laser Program Annual Report ${ }_{4}$ UCRL-50021-82, C. D. Hendricks, scientific editor, 7.34-38 (University of California, Lawrence Livermore National Laboratory, Aug 1983); ibid., UCRL-50021-81, E. V. George, scientific editor, 7.20-4 (Aug. 1982). 
22. W. L. Smith, "Absolute two-photon absorption coefficient at $355 \mathrm{~nm}$ in UV glasses," Post-deadline paper, Conference on Lasers and Electro-Optics, Washington, D. C., June 10-12, 1981.

23. J. Wong and C. A. Angell, Glass Structure by Spectroscopy (Marcel Dekker, Inc., New York, 1976).

24. Nd-Doped Laser Glass Spectroscopic and Physical Properties, Handbook LLNLM-95, S. Stokowski, R. A. Saroyan, and M. J. Weber, editors (University of California, Lawrence Livermore National Laboratory, 1978).

25. C. B. Layne, W. H. Lowdermilk, and M. J. Weber, "Quantum efficiency: nonradiative decay by multiphonon emission," in Laser Program Annual Report1975, UCRL-50021-75, A. J. Glass, scientific editor, 204-5 (University of California, Lawrence Livermore National Laboratory, Mar. 1976).

26. Douglas W. Hall, Fluorescence Line-Narrowing Studies of Rare Earths in Disordered Solids, PhD Thesis, University of California at Davis (1982), p. 66; B. R. Judd, Optical absorption intensities of rare-earth ions, Phys. Rev. 127(3), 750-61 (1 Aug. 1962); G. S. Ofelt, "Intensities of crystal spectra of rare-earth ions," J. Chem. Phys. 37(3), 511-520 (1 Aug 1962).

27. N. L. Boling, Alexander J. Glass, and A. Owyoung, "Empirical relationships for predicting nonlinear refractive index changes in optical solids," IEEE $J$. Quantum Electr. 14(8), 601-8 (Aug 1978).

28. W. T. White, III, W. L. Smith, and D. Milam, "Direct measurement of the nonlinear refractive index coefficient $\gamma$ at $355 \mathrm{~nm}$ in fused silica and in BK-10 glass," Opt. Lett. 9(1) 10-12 (Jan 1984).

29. Y. Bae, J. Song, and Y. B. Kim, "Photoacoustic study of two-photon absorption in hexagonal ZnS," J. Appl. Phys. 53(1), 615-19 (Jan. 1982). 
Chapter 6

\section{SUMMARY AND CONCLUSIONS}

\subsection{Summary and Conclusions.}

In this work we have theoretically and experimentally investigated two-photon absorption in highly transparent solids. Our main conclusions are (1) that empirical pseudopotential methods can be used to compute accurate values of $\beta$ in large band-gap zinc blende materials like $\mathrm{ZnS}$ and (2) that thermal lensing is sufficiently sensitive to measure two-photon absorption coefficients on the order of $1 \mathrm{~cm} / \mathrm{TW}$.

In Chapter 2, we thoroughly reviewed thip theory of two-photon absorption in zinc blende materials, finding why various thecries disagreed. Almost always, mathematical or physical modeling errors were to blame. When the errors were corrected, the models agreed with each other to within $50 \%$, an insignificant figure in light of the variability of many nonlinear optical measurements. Unfortunately, those models could not predict the polarization dependence or the correct dispersion of the two-photon absorption coefficient, and they were a factor of 3 or more lower than well calibrated experimental values.

This led us to try a relatively unexplored technique for predicting two-photon absorption in solids, an empirical pseudopotential method that was discussed in Chapter 3. The pseudopotential parameters for $\mathrm{ZnS}$ were determined from the work of Cohen and Bergstresser. ${ }^{1}$ In checking our work, we found that we were able to improve upon Cohen's calculations of linear optical reflectance in the range 0-7 $\mathrm{eV}$. This encouraged us to proceed with the nonlinear calculation. Although our calculations were for absolute zero temperature, we simulated the effect of temperature by moving the absorption edge from the $0 \mathrm{~K}$ band edge to the $300 \mathrm{~K}$ band edge. After this 0.18-eV shift, our predictions agreed with experimental measurements 
within $5 \%$ near the band edge and and within $15 \%$ over a $1.5 \mathrm{eV}$ range. Above 5 $\mathrm{eV}$, the theoretical and experimental results diverged. It was not clear whether the disagreement was a modeling error or a measuring error. This extremely good agreement between theory and measurement suggests that the empirical pseudopotential method has promising unexplored applications in modeling nonlinear physical phenomena. Calculating nonlinear optical properties with pseudopotentials should be further investigated, because it appears to offer a reasonable way to estimate material parameters that are of fundamental importance in integrated optics, fiber optics, and high-power lasers.

In Chapter 4, we discussed the theory of pulsed thermal lensing. The new result of that chapter was to derive a theory that accounted for the spherical aberration in a thin Gaussian-shaped lens. This theory was necessary for analyzing our experimental data, since our pump and probe beams had comparable diameters.

In Chapter 5 we used thermal lensing to measure two-photon absorption. Our measured results in $\mathrm{ZnS}$ agreed with the calibrated data of Bae et al. ${ }^{2}$ within $\pm 15 \%$. The $\mathrm{TiO}_{2}$ measurements agreed with previous measurements of Waff and Park ${ }^{3}$ to within $20 \%$, except for a broad absorption peak that appeared in our data. Waff and Park's results suggested that the peak should have been absent for the crystal orientation and polarization that we used. For a different orientation, however, a peak should have occurred exactly where ours did, according to Waff and Park. Whether the peak was an artifact of misalignment or whether it was a consequence of using a different sample or higher temperature was not determined. Agreement with previous data was satisfactory over the half of the spectrum that lay above the peak.

We then applied the technique to several glasses. At high intensities the absorption increased nonlinearly in most samples. The effective coefficient of two-photon 
absorption was of the order of $1 \mathrm{~cm} / \mathrm{TW}$. Although further work is required to determine whether the nonlinearities that were observed were due to two-photon absorption or to two-step absorption, indirect evidence suggests that the source of the nonlinear absorption was impurities and defects. This indirect evidence includes the fact that nonlinear absorption was always preceded by linear absorption at lower fluences $\left(\alpha \approx 10^{-3} \mathrm{~cm}^{-1}\right)$, that impurity excited-state lifetimes can be long compared to the laser pulse width, that the intrinsic absorption edges of the glasses were probably at energies greater than $2 \hbar \omega$ in our tests, and that there was no clear relationship between the measured nonlinearities and any other observed property of the samples. The uncertainty of the results for the glasses was higher than for the crystals. This was primarily a result of the lower signal of the measurements in glass; both the absorption coefficient and the thermo-optic coefficient of the crystals were higher than those of the glasses. The most important conclusion to be drawn from the measurements is that the thermal lensing technique used here was sufficiently sensitive to quantify the very weak nonlinear absorption in highly transparent glasses. 


\section{References}

1. M. L. Cohen and T. K. Bergstresser, "Band structures and pseudopotential form factors for fourteen semiconductors of the diamond and zinc-blende structures," Phys. Rev. 141(2), 789-96 (Jan. 1966).

2. Y. Bae, J. J. Song, and Y. B. Kim, "Photoacoustic study of two-photon absorption in hexagonal ZnS," J. Appl. Phys. 53(1), 615-19 (Jan. 1982).

3. H. S. Waff and K. Park, "Structure in the spectrum of the two-photon absorption spectrum of $\mathrm{TiO}_{2}$ (rutile)," Phys. Lett. 32A(2), 109-10 (15 June 1970). 
Appendix A

\section{BLOCH'S DETERMINANTAL FORM OF SCHRÖDINGER'S} EQUATION IN CRYSTALS

The one-election wave equation of a crystal is

$$
-\frac{\hbar^{2}}{2 m} \nabla^{2} \psi+U(\vec{r}) \psi=E \psi
$$

where $\hbar$ is Planck's constant, $\mathrm{m}$ is the electron mass, and $E$ is the electron energy. Neglecting spin-orbit interactions, the potential energy $U(\vec{r})$ may be written as a superposition of effective atomic potentials $U_{j}$.

$$
U(\vec{r})=\sum_{n} \sum_{j} U_{j}\left(\vec{r}-\vec{R}_{n}-\vec{\rho}_{j}\right)
$$

where $\vec{R}_{n}$ is the position of the center of the n-th unit cell and $\vec{\rho}_{j}$ is the position of the given atomic core relative to the center of a cell. Near the core of atom number $j, U_{j}$ equals the potential of the isolated atom $j$, but away from the core it vanishes rapidly with distance due to electrostatic screening.

The function $U(\vec{r})$ is translationally invariant from one unit cell to the next. A natural set of basis functions for expanding it is a set of plane waves of the form $\exp \left(i \vec{G}_{m} \cdot \vec{r}\right)$, where $\vec{G}_{m}$ is an arbitrary reciprocal lattice vector. $\vec{G}_{m}$ is defined so that for an arbitrary translation $\vec{R}_{n}$ from the center of one unit cell to the center of another,

$$
\vec{G}_{m} \cdot \vec{R}_{n}=2 \pi M
$$

where $M$ is some integer. This property results if $\vec{G}_{m}$ is defined as a linear combination of vectors $\vec{Q}_{i}$,

$$
\vec{G}_{m}=m_{1} \vec{Q}_{1}+m_{2} \vec{Q}_{2}+m_{3} \vec{Q}_{3}
$$


where $m_{1}, m_{2}$, and $m_{3}$ are integers and where

$$
\begin{aligned}
& \vec{Q}_{1}=\frac{2 \pi}{\Omega} \vec{a}_{2} \times \vec{a}_{3} \\
& \vec{Q}_{2}=\frac{2 \pi}{\Omega} \vec{a}_{3} \times \vec{a}_{1} \\
& \vec{Q}_{3}=\frac{2 \pi}{\Omega} \vec{a}_{1} \times \vec{a}_{2}
\end{aligned}
$$

and

$$
\Omega=\vec{a}_{1} \cdot \vec{a}_{2} \times \vec{a}_{3} .
$$

The vectors $\vec{a}_{1}, \vec{a}_{2}$, and $\vec{a}_{3}$ are "primitive lattice vectors," and $\Omega$ is the volume of a unit cell. The translation $\vec{R}_{n}$ is defined as a linear combination of the primitive lattice vectors:

$$
\vec{R}_{n}=n_{1} \vec{a}_{1}+n_{2} \vec{a}_{2}+n_{3} \vec{a}_{3}
$$

where $n_{1}, n_{2}$, and $n_{3}$ are integers.

Following Ashcroft and Mermin, ${ }^{1}$ the crystal is imagined to be built up from $N_{1}$ unit cells in the $\vec{a}_{1}$ direction, $N_{2}$ unit cells in the $\vec{a}_{2}$ direction, and $N_{3}$ unit cells in the $\vec{a}_{3}$ direction. The volume of the crystal, $V$, equals $N_{1} N_{2} N_{3} \Omega$. The wave function $\psi$, being spatially periodic with periods $N_{1} \vec{a}_{1}, N_{2} \vec{a}_{2}$, and $N_{3} \vec{a}_{3}$ in the three independent directions, may therefore be expanded in plane waves of the form $\exp \left(i \vec{K}_{\ell} \cdot \vec{r}\right)$, where the wave vector $\vec{K}_{\ell}$ is given by

$$
\vec{K}_{\ell}=\frac{\ell_{1} \vec{Q}_{1}}{N_{1}}+\frac{\ell_{2} \vec{Q}_{2}}{N_{2}}+\frac{\ell_{3} \vec{Q}_{3}}{N_{3}}
$$

and where $\ell_{1}, \ell_{2}$, and $\ell_{3}$ are integers. That is,

$$
\psi=\sum_{\vec{K}} C(\vec{K}) \exp (i \vec{K} \cdot \vec{r})
$$

Using Eqs. (A.2) and (A.9) in Eq. (A.1) one has

$$
0=\sum_{\vec{K}}\left[\left(\frac{\hbar^{2} K^{2}}{2 m}-E\right)+\sum_{n} \sum_{j} U_{j}\left(\vec{r}-\vec{R}_{r}-\vec{\rho}_{j}\right)\right] C(\vec{K}) \exp (i \vec{K} \cdot \vec{r}) .
$$


Equation (A.10) can be reduced to a set of coupled linear equations not involving $\vec{r}$. Upon multiplying by $\exp \left(-i \vec{K}^{\prime} \cdot \vec{r}\right)$ and integrating over the volume $V$ of the crystal, one gets

$$
\begin{aligned}
0=\sum_{\vec{K}} & {\left[\left(\frac{\hbar^{2} K^{2}}{2 m}-E\right) \delta_{\vec{K} \vec{K}^{\prime}}+\sum_{n} \sum_{j} \frac{1}{V} \int_{V} d^{3} \vec{r} \exp \left(-i \vec{K}^{\prime} \cdot \vec{r}\right)\right.} \\
& \left.\times U_{j}\left(\vec{r}-\vec{R}_{n}-\vec{\rho}_{j}\right) \exp (i \vec{K} \cdot \vec{r})\right] C(\vec{K})
\end{aligned}
$$

where

$$
\delta_{\bar{K} \bar{K}^{\prime}}= \begin{cases}1, & \vec{K}=\vec{K}^{\prime} \\ 0, & \text { otherwise }\end{cases}
$$

Since $U_{j}$ is negligibly small at the edges of the volume $V$, except for atoms within a few ionic radii of the boundaries, the bounds on the integral in Eq. (A.11) may be extended over all space. The bounds on the summations, however, remain confined to all unit cells within $V$. For macroscopic volumes $V$, the number $N$ of unit cells is so large that from Eq. (A.3) the following approximation is nearly exact:

$$
\frac{1}{N} \sum_{n} \exp \left(i\left(\vec{K}-\vec{K}^{\prime}\right) \cdot \vec{R}_{n}\right)= \begin{cases}1, & \text { if a reciprocal lattice vector } \vec{G} \text { exists } \\ & \text { such that } \vec{K}-\vec{K}^{\prime}=\vec{G} . \\ 0, & \text { otherwise. }\end{cases}
$$

Equation (A.13) shows that only those wave vectors which equal $\vec{K}^{\prime}$ to within a reciprocal lattice vector can contribute to the summation over $\vec{K}$ in Eq. (A.11). Therefore, let us restrict $\vec{K}$ to lie in the first Brillouin zone (See Appendix C.) and replace the summation over $\vec{K}$ with a summation over reciprocal lattice vectors $\vec{G}$. Equation (A.11) becomes

$$
\begin{aligned}
0=\sum_{\vec{G}} & {\left[\left(\frac{\hbar^{2}(\vec{K}-\vec{G})^{2}}{2 m}-E\right) \delta_{\vec{K}^{\prime}, \vec{K}-\vec{G}}\right.} \\
& \left.+\sum_{j} V_{j}\left(\vec{K}^{\prime}+\vec{G}, \vec{K}\right) S_{j}\left(\vec{K}^{\prime}-\vec{K}+\vec{G}\right)\right] C(\vec{K} \cdot \vec{G})
\end{aligned}
$$

The functions $V_{j}$ and $S_{j}$ are respectively the "atomic form factor" and the "structure factor," defined as

$$
V_{j}\left(\vec{K}^{\prime}, \vec{K}\right)=\frac{n_{j}}{\Omega} \int_{-\infty}^{\infty} d^{3} \vec{r} \exp \left(-i \vec{K}^{\prime} \cdot \vec{r}\right) U_{j}(\vec{r}) \exp (i \vec{K} \cdot \vec{r})
$$


and

$$
S_{j}\left(\vec{K}^{\prime}-\vec{K}\right)=\frac{1}{n_{j}} \sum_{\vec{e}_{j}} \exp \left(i\left(\overrightarrow{K^{\prime}}-\vec{K}\right) \cdot \vec{\varrho}_{j}\right)
$$

where $n_{j}$ denotes the number of atoms of type $j$ per unit cell and $j$ no longer indexes each atom but now indexes each different kind of atom in a unit cell. By Eq. (A.13), we set $\vec{K}^{\prime}=\vec{K}-\vec{G}^{\prime}$ for a suitable $\vec{G}^{\prime}$ and obtain a set of coupled linear equations for all reciprocal lattice vectors $\vec{G}^{\prime}$ :

$$
0=\sum_{\vec{G}}\left[\left(\frac{\hbar^{2}(\vec{K}-\vec{G})^{2}}{2 m}-E\right) \delta_{\vec{G}, \vec{G}^{\prime}}+\sum_{j} V_{j}\left(\vec{G}, \vec{G}^{\prime}\right) S_{j}\left(\vec{G}-\vec{G}^{\prime}\right)\right] C(\vec{K}-\vec{G})
$$

For a non-trivial solution to Eq. (A.17), the determinant of the coefficients of the $C(\vec{K}-\vec{G})$ must vanish. This gives Bloch's deteiminantal form of Schrödinger's Equation:

$$
0=\operatorname{det}\left[\left(\frac{\hbar^{2}(\vec{K}-\vec{G})^{2}}{2 m}-E\right) \delta_{\vec{G}, \vec{G}^{\prime}}+\sum_{j} V_{j}\left(\vec{G}, \vec{G}^{\prime}\right) S_{j}\left(\vec{G}-\vec{G}^{\prime}\right)\right]
$$

\section{Reference}

1. N. W. Ashcroft and N. D. Mermin, Solid State Physics (Saunders College, Philadelphia, 1976). 
Appendix B

\section{RECIPROCAL LATTICE VECTORS OF A FACE-CENTERED \\ CUBIC LATTICE}

Figure B-1 illustrates a conventional cubic cell in a face centered cubic Bravais lattice. The cube has an edge length of $\mathrm{b}$, and the Cartesian axes $\hat{i}, \hat{j}$, and $\hat{k}$ are normal to each of the three pairs of opposing faces. The vectors $\vec{a}_{1}, \vec{a}_{2}$, and $\vec{a}_{3}$, are three linearly independent, primitive translation vectors of the lattice.

$$
\begin{aligned}
& \vec{a}_{1}=(\hat{\jmath}+\hat{k}) \frac{b}{2} \\
& \vec{a}_{2}=(\hat{k}+\hat{\imath}) \frac{b}{2} \\
& \vec{a}_{3}=(\hat{\imath}+\hat{\jmath}) \frac{b}{2}
\end{aligned}
$$

In terms of these three vectors, the fundamental reciprocal lattice vectors $\vec{Q}_{1}, \vec{Q}_{2}$, and $\vec{Q}_{3}$ are defined as

$$
\begin{aligned}
& \vec{Q}_{1}=\frac{2 \pi \vec{a}_{2} \times \vec{a}_{3}}{\vec{a}_{1} \cdot \vec{a}_{2} \times \vec{a}_{3}}=(-\hat{\imath}+\hat{\jmath}+\hat{k}) 2 \pi / b \\
& \vec{Q}_{2}=\frac{2 \pi \vec{a}_{3} \times \vec{a}_{1}}{\vec{a}_{1} \cdot \vec{a}_{2} \times \vec{a}_{3}}=(\hat{\imath}-\hat{\jmath}+\hat{k}) 2 \pi / b \\
& \vec{Q}_{3}=\frac{2 \pi \vec{a}_{1} \times \vec{a}_{2}}{\vec{a}_{1} \cdot \vec{a}_{2} \times \vec{a}_{3}}=(\hat{\imath}+\hat{\jmath}-\hat{k}) 2 \pi / b
\end{aligned}
$$

An arbitrary reciprocal lattice vector for the fcc lattice is a linear combination of the form

$$
\vec{G}_{m}=m_{1} \vec{Q}_{1}+m_{2} \vec{Q}_{2}+m_{3} \vec{Q}_{3}
$$

where $m_{1}, m_{2}$, and $m_{3}$ are integers. In terms of the Cartesian unit vectors $\hat{\imath}, \hat{\jmath}$, and $\hat{k}$, the vector $\bar{G}_{m}$ is given by

$$
\vec{G}_{m}=\left(M_{1} \hat{\imath}+M_{\imath} \hat{\jmath}+M_{3} \hat{k}\right) 2 \pi / b,
$$

where 


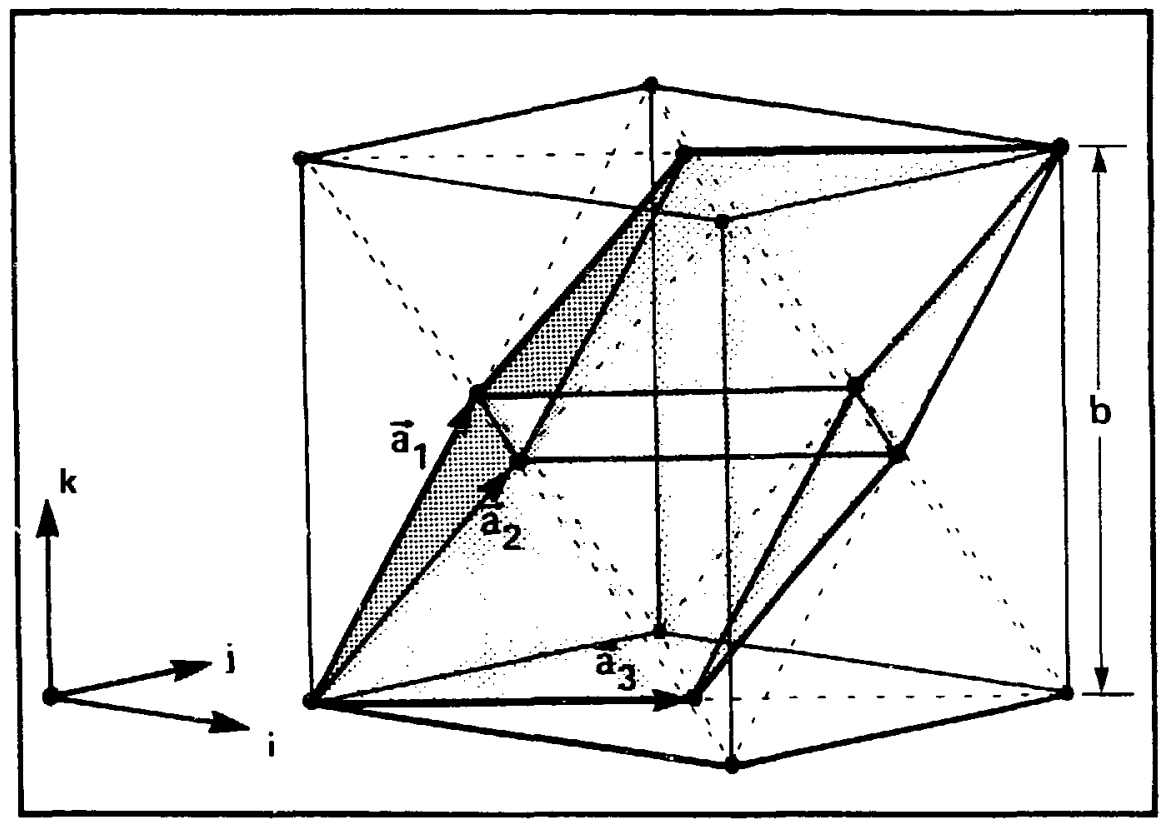

Figure B-1. Face-Centered Cubic Lattice. The shaded region is a unit cell. 


$$
\begin{aligned}
& M_{1}=-m_{1}+m_{2}+m_{3} \\
& M_{2}=m_{1}-m_{2}+m_{3} \\
& M_{3}=m_{1}+m_{2}-m_{3}
\end{aligned}
$$

Since $M_{1}-M_{2}, M_{2}-M_{3}$, and $M_{3}-M_{1}$ are all even integers, the important simplification follows that $M_{1}, M_{2}$, and $M_{3}$ are either all even or all odd. Table B-1 gives the first few fcc reciprocal lattice vectors in order of ascending magnitude. 


\begin{tabular}{|clcc|}
\hline $\mathrm{m}$ & $\begin{array}{l}\vec{G}_{m} / \frac{2 \pi}{b} \\
(i, j, k)\end{array}$ & $\left|\vec{G}_{m}\right|^{2} /\left(\frac{2 \pi}{b}\right)^{2}$ & Degeneracy \\
\hline 1 & $(0,0,0)$ & 0 & 1 \\
2 & $( \pm 1, \pm 1, \pm 1)$ & 3 & 8 \\
3 & $( \pm 2,0,0)$ & 4 & 6 \\
4 & $( \pm 2, \pm 2,0)$ & 8 & 12 \\
5 & $( \pm 3, \pm 1, \pm 1)$ & 11 & 24 \\
6 & $( \pm 2, \pm 2, \pm 2)$ & 12 & 8 \\
7 & $( \pm 4,0,0)$ & 16 & 6 \\
8 & $( \pm 3, \pm 3, \pm 1)$ & 19 & 24 \\
9 & $( \pm 4, \pm 2,0)$ & 20 & 24 \\
10 & $( \pm 4, \pm 2, \pm 2)$ & 24 & 24 \\
11 & $( \pm 5, \pm 1, \pm 1)$ & 27 & 27 \\
12 & $( \pm 3, \pm 3, \pm 3)$ & 24 & \\
\hline
\end{tabular}

Table B-1. The First 175 FCC Reciprocal Lattice Vectors in Order of Ascending Magnitude. All possible permutations of the sign and order of indices $(i, j, k)$ are also valid reciprocal lattice vectors. 
Appendix C

\section{FIRST BRILLOUIN ZONE OF THE FCC LATTICE}

This section explains how the Brillouin Zone was discretized in the zinc blende calculations in Chapter 3. The first Brillouin zone is defined as a "Wigner-Seitz cell in reciprocal lattice space. ${ }^{11}$ It is the set of all points closer to the origin than to any other reciprocal lattice point. To construct it, one locates the largest simple closed volume that contains the origin and that is not sliced by a perpendicular bisector plane of any reciprocal lattice vector $\vec{G}=0$. For the fcc space lattice, the perpendicular bisectors of the reciprocal lattice vectors $( \pm 1, \pm 1, \pm 1)( \pm 2,0,0)$, $(0, \pm 2,0)$ and $(0,0, \pm 2)$ bound the first Brillouin zone. The units of these vectors are $2 \pi / b$, where $b$ is the width of a single face-centered cube. Figure $C-1$ depicts the resulting truncated octahedron, which is given by the set of all wave vectors that satisfy the following two simultaneous criteria:

$$
\left|K_{x}\right|,\left|K_{y}\right|,\left|K_{z}\right|<\frac{2 \pi}{b}
$$

and

$$
\left|K_{x}\right|+\left|K_{y}\right|+\left|K_{z}\right|<\frac{3 \pi}{b}
$$

When one calculates quantum mechanical properties, symmetry considerations reduce the need to compute the wave function at every point in the Brillouin zone. For the point group $\overline{4} 3 \mathrm{~m}\left(T_{d}\right)$, which characterizes the zinc blende structure, the three operations of (1) $2 \pi / 3$ rotation about the $(1,1,1)$ axis, $(2) 2 \pi / 4$ roto-inversion about the $(1,0,0)$ axis, and (3) reflection about the diagonal bisector plane of $(1,0,0)$ and $(0,1,0)$ can, by repeated application, map all points in the Brillouin zone into a segment whose volume is $1 / 24$ of the whole zone. That segment, which we define as the "irreducible Brillouin zone" of the point group $\overline{4} 3 \mathrm{~m}$, consists of two diametrically 


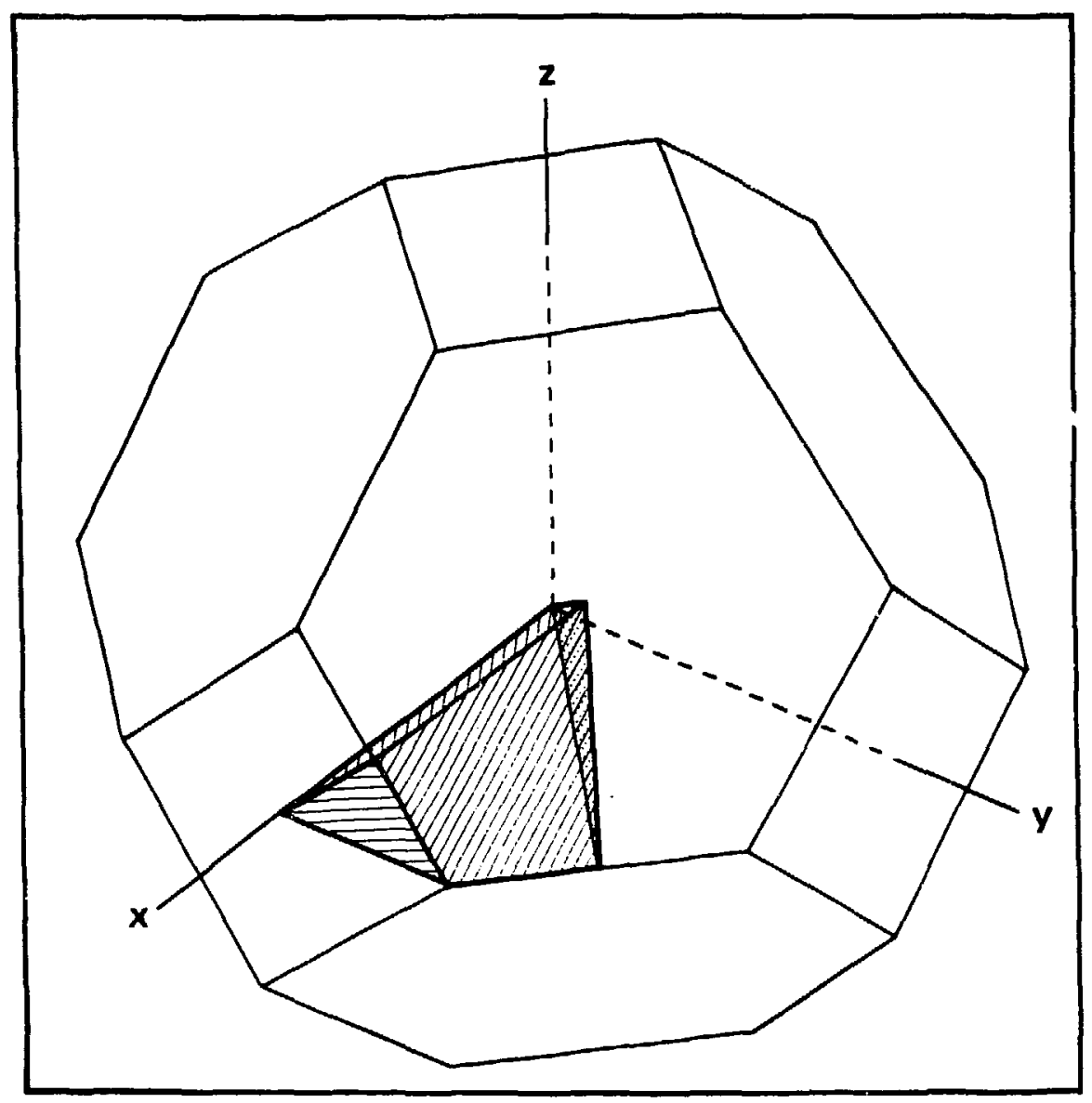

Figure C-1. Half of the Irreducible Brillouin Zone of the Zinc Blende Structure. 
opposite sectors:

$\frac{2 \pi}{b} \geq K_{x} \geq K_{y} \geq K_{z} \geq 0$

and

$\frac{2 \pi}{b} \geq-K_{x} \geq-K_{y} \geq-K_{z} \geq 0$

Figure C-1 illustrates one of these two sectors, which is $1 / 48$ of the volume of the Brillouin zone, $V_{B Z}$.

Figure C-2 illustrates the grid used to sample the irreducible Brillouin zone (IBZ) in the computations of Chapter 3. A uniform array of small cubes was superimposed on the IBZ. In each cube, the corner that was farthest from the origin was chosen as representative of all points inside the cube. In addition to Eqs. (C.1) and (C.2), the following criteria define the sampling of wave vectors:

$$
\begin{aligned}
\left|K_{z}\right| & \geq \Delta K \\
\triangle K_{x} & =\Delta K_{y}=\Delta K_{z}=\Delta K \\
(\triangle K)^{3} & =\frac{V_{B Z}}{48 N_{p t s}} \\
V_{B Z} & =4\left(\frac{2 \pi}{b}\right)^{3} \\
\vec{K}_{m n p} & =(m, n, p) \triangle K ;
\end{aligned}
$$

where $N_{p t s}$ is approximately the number of points in one sector and where $m, n$, and $p$ are integers.

\section{Reference}

1. N. W. Ashcroft and N. D. Mermin, Solid State Physics (Saunders College, Philadelphia, 1976). 


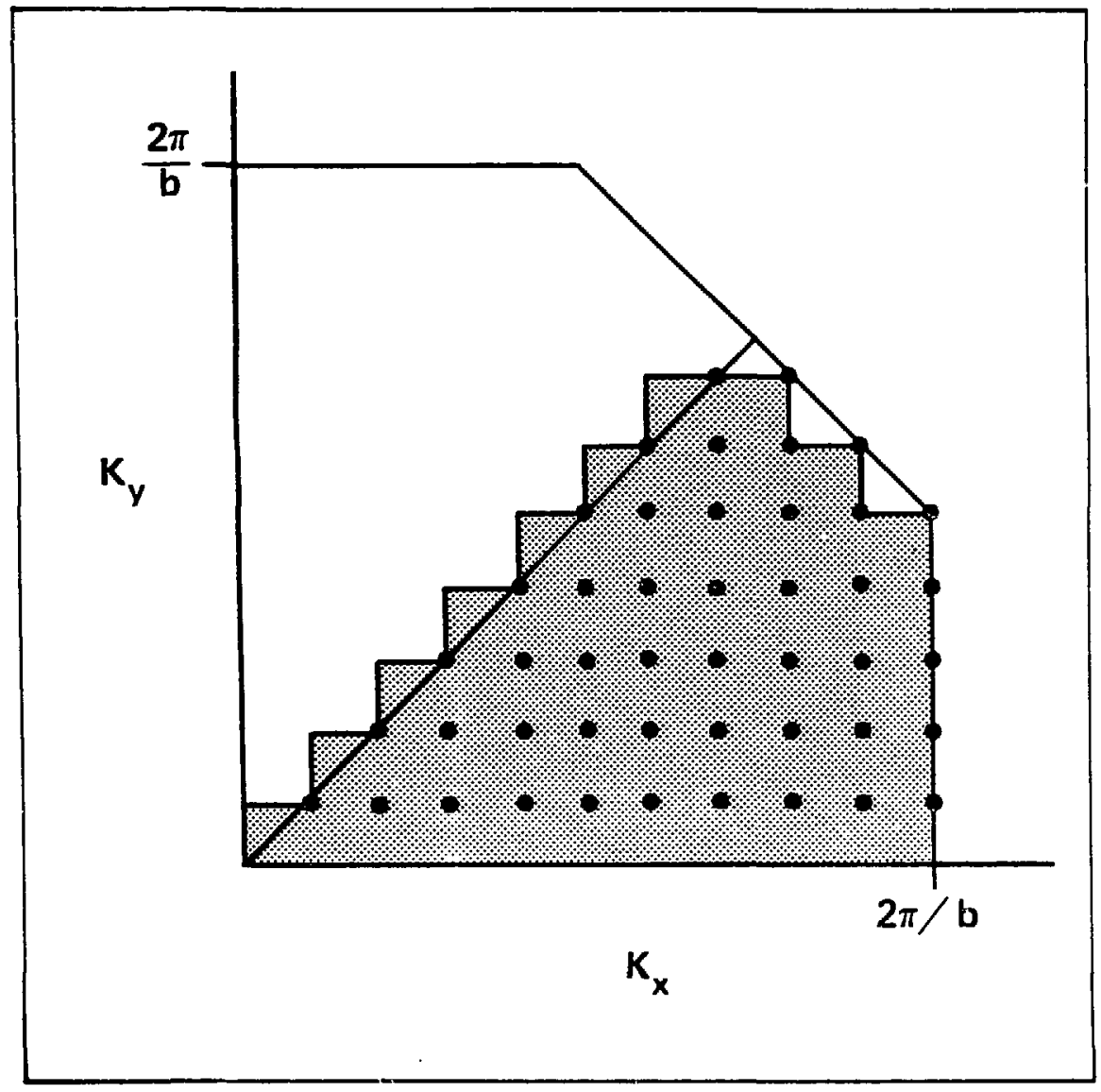

Figure C-2. Discretized $1 \mathrm{BL}$ of Figure $\mathrm{C}-1$. The view is looking down the $z$-axis. 
Appendix D

\section{INDIRECT TWO-PHOTON ABSORPTION IN CUBIC Z̈nS}

This appendix compares indirect two-photon absorption to direct two-photon absorption in $\mathrm{ZnS}$ over the frequency range $E_{G}<2 \hbar \omega<2 E_{i,}$. It is found that indirect two-photon absorption is probably not important, contrary to the conjecture of Yablonovitch et al. ${ }^{1}$ Indirect two-photon absorption is a two-step process. First an intense optical wave generates a second-harmonic wave in an acentric material. Then the second-harmonic wave mediates the transfer of energy from the intense wave to the material. The material is assumed to have a significant linear absorption cuefficient at the second-harmonic frequency.

Two cases are of interest, phase-matched and phase-mismatched harmonic generation. In the case of phase-matched harmonic generation, a strong harmonic wave builds up. However, due to losses at the harmonic frequency, which are assumed large, the harmonic wave attenuates. Power thus flows from the fundamental wave, through the second harmonic wave, and into the material. In materials of cubic ZnS structure, phase-matched second harmonic generation cannot ordinarily occur. This is because the linear dielectric constant is isotropic in these materials, and so dispersion prevents phase matching. ${ }^{2}$ That is, the linear dielectric constant at the frequency of the fundamental wave cannot equal the linear dielectric constant at the second-harmonic frequency for any orientation of fields in such materials. Therefore, we shall co'sider only indirect two-photon absorption due to phase-mismatched frequency doubling.

In the case of phase-mismatched harmonic generation, a strong harmonic wave never builds up. Instead, a weak polarization density is created, and this polarization density acts like a current-source (cf. Eq. (2.59)) that beats against the 
incident wave. The result is that another polarization density is induced, thirdorder in the incident field strength and with the same frequency as the incident wave. The effective third-order nonlinear susceptibility in this case is proportional to the product of two second-order susceptibilities. The imaginary part of this effective third-order susceptibility corresponds to an indirect wwo-photon absorption coefficient. Power flows from the fundamental wave, through the weak harmonic wave, and back to the fundamental. In the process, the absorption coefficient of the surrounding medium is perturbed, and part of the power in the fundamental wave is siphoned arto the medium.

Indirect two-photon absorption can be important even for phase-mismatched harmonic generation. This is because the phase mismatch that is incurred when the incident waves beat to form the harmonic wave is always undone when the harmonic wave subsequently beats against the incident waves to yield a third-order polarization. That is, the indirect third-order polarization is always phase-matched to the incident wave, irrespective of the phase mismatch between the fundamental and the second-harmonic wave. However, as Yablonovitch has shown, ${ }^{1}$ the effective third-order susceptibility does have a component that is proportional to the reciprocal of the phase mismatch. Thus, indirect two-photon absorption should increase as the harmonic conversion becomes more efficient.

To analyze indirect two-photon absorption, we resort to the slowly-varyingplane-wave approximation. At every point, the total macroscopic electric field is comprised of contributions at $\pm \omega$ and $\pm 2 \omega$. Let us assume that all components travel in the same direction. Such constraints are valid if the incident beam is perpendicular to the boundary of the nonlinear medium. ${ }^{3}$ The nonlinear medium is assumed to fill all of space on one side of a plane, and vacuum is assumed to fill all space on the other side. This assumption allows one to neglect back-surface 
reflections.

The components of the electric field at $\omega$ and $2 \omega$ are taken as $\vec{E}(\omega)$ and $\vec{E}(2 \omega)$, where

$$
\vec{E}(\omega)=\vec{\varepsilon}_{1}(\varsigma) e^{i k_{1} \varsigma}
$$

and

$$
\vec{E}(2 \omega)=\vec{\varepsilon}_{2}(\varsigma) e^{i k_{2} \zeta}
$$

The coordinate $\zeta$ denotes position along the direction of propagation, $\varepsilon_{1}$ and $\varepsilon_{2}$ are slowly-varying amplitudes and $\vec{k}_{1}$ and $\vec{k}_{2}$ are the optical wave vectors at $\omega$ and $2 \omega$, respectively.

The nonlinear polarizations $\vec{P}^{N L}(\omega)$ and $\vec{P}^{N L L}(2 \omega)$ may be written in the slowlyvarying amplitude approximation as

$$
\begin{gathered}
\vec{P}^{N L}(\omega)=\vec{P}_{1}(\varsigma) e^{i k_{1 \zeta}} \\
\vec{P}^{N L}(2 \omega)+\vec{P}_{2}(\varsigma) e^{i k_{2 \zeta}}
\end{gathered}
$$

where

$$
\vec{P}_{1}=\chi^{(3)}(-\omega ; \omega, \omega,-\omega)\left|\overrightarrow{\mathcal{E}}_{1} \overrightarrow{\mathcal{E}}_{1} \overrightarrow{\mathcal{E}}_{1}+\chi^{(2)}(-\omega ;-\omega, 2 \omega)\right| \overrightarrow{\mathcal{E}}_{1}^{*} \overrightarrow{\mathcal{E}}_{2} e^{i \Delta k_{5}}
$$

and

$$
\vec{P}_{2}=x^{(2)}(-2 \omega ; \omega, \omega) \mid \vec{\varepsilon}_{1} \vec{\varepsilon}_{1}
$$

The vertical bars in Eqs. (D.3) denote multiple dot products, or tensor contraction, as in Butcher. ${ }^{4}$ Subscripts have been omitted from the nonlinear susceptibility tensors for ease of notation.

In Eq. (D.3a), $\Delta k$ is the mismatch in the wavevectors $2 \vec{k}_{1}$ and $\vec{k}_{2}$,

$$
\Delta k \equiv k_{2}-2 k_{1}
$$

The terms contributing to $\vec{P}_{1}$ include the direct two-photon absorption term which involves $\chi^{(3)}$, plus the indirect two-photon absorption term, which involves $\chi^{(2)}$. 
The term contributing to $\vec{P}_{2}$ is the frequency-doubling term, which involves only $\chi^{(2)}$. The amplitude of $\overrightarrow{\mathcal{E}}_{2}$ is assumed to be too weak to contribute directly to $\chi^{(3)}$ processes in either $\vec{P}_{1}$ or $\vec{P}_{2}$.

From these definitions, the wave equation may be written for each separate Fourier component as follows:

$$
\begin{aligned}
& 2 i k_{1}\left(\overrightarrow{\mathcal{E}}_{1}^{\perp}\right)^{\prime}+\left(k_{1}^{2}-\left(\frac{\omega}{c}\right)^{2} \epsilon(\omega)\right) \overrightarrow{\mathcal{E}}_{1}^{\perp}=\left(\frac{\omega}{c}\right)^{2} 4 \pi \vec{P}_{1}^{\perp} \\
& 2 i k_{2}\left(\overrightarrow{\mathcal{E}}_{2}^{\perp}\right)^{\prime}+\left(k_{2}^{2}-\left(\frac{2 \omega}{c}\right)^{2} \epsilon(2 \omega)\right) \overrightarrow{\mathcal{E}}_{2}^{\perp}=\left(\frac{2 \omega}{c}\right)^{2} 4 \pi \vec{P}_{2}^{\perp} .
\end{aligned}
$$

In these expressions, $\epsilon(\omega)$ and $\epsilon(2 \omega)$ denote the linear dielectric constants at $\omega$ and $2 \omega$, respectively. Since $\vec{\nabla} \cdot\left(\epsilon \vec{E}+4 \pi \vec{P}^{N L}\right)=0$, one has

$$
\overrightarrow{\mathcal{E}}_{1}^{\|}=-4 \pi \vec{P}_{1}^{\|} / \epsilon(\omega)
$$

and

$$
\overrightarrow{\mathcal{E}}_{2}^{\|}=-4 \pi \vec{P}_{2}^{\|} / \epsilon(2 \omega)
$$

The superscripts $\|$ and $\perp$ respectively denote the mutually exclusive longitudinal (irrotational) and transverse (divergenceless) components of a vector field, and the prime denotes partial derivative with respect to $\zeta$.

Because the second-harmonic generation is not phase-matched, the derivative of $\overrightarrow{\mathcal{E}}_{2}^{\perp}$ in Eq. (D.5b) may be neglected with respect to the presumably more rapidly varying term $\left(k_{2}^{2}-(2 \omega / c)^{2} \epsilon(2 \omega)\right) \vec{\varepsilon}_{2}^{\perp}$. Together with the definition of the new parameter $\epsilon_{2}$

$$
\epsilon_{2} \equiv\left(\frac{c k_{2}}{2 \omega}\right)^{2}
$$

one obtains from Eq. (D.5b) a relation for $\overrightarrow{\mathcal{E}}_{2}^{\perp}$ in terms of $\vec{P}_{2}^{\perp}, \epsilon_{2}$, and $\epsilon(2 \omega)$. This allows elimination of $\overrightarrow{\mathcal{E}}_{2}$ from Eq. (D.3a) by writing

$$
\overrightarrow{\mathcal{P}}_{1}=\overrightarrow{\mathcal{P}}_{1}^{\text {direct }}+\overrightarrow{\mathcal{P}}_{1}^{\text {indirect }}
$$

where 


$$
\vec{P}_{1}^{\text {direct }}=\chi^{(3)}(-\omega ; \omega, \omega,-\omega) \mid \vec{\varepsilon}_{1} \vec{\varepsilon}_{1} \vec{\varepsilon}_{1}^{*}
$$

and

$$
\vec{P}_{1}^{\text {indirect }}=-4 \pi \chi^{(2)}(-\omega ;-\omega, 2 \omega) \mid \overrightarrow{\mathcal{E}}_{1}^{*}\left(\frac{\vec{P}_{2}^{\|}}{\epsilon(2 \omega)}+\frac{\vec{P}_{2}^{\perp}}{\epsilon(2 \omega)-\epsilon_{2}}\right),
$$

and where $\vec{P}_{2}$ is given by Eq. (D.3b) only in terms of $\vec{\varepsilon}_{1}$. In Eqs. (D.9) the absence of an exponential term involving $\Delta k_{\zeta}$ shows that the indirect contribution to $\vec{P}_{1}$ is indeed phase-matched, as asserted earlier. Additionally, for normal incidence, electromagnetic boundary conditions ensure $k_{2}=2 k_{1}{ }^{3}$ Therefore, from Eq. (D.7) $\epsilon_{2}=c^{2} k_{1}^{2} / \omega_{1}^{2}$, which for weak nonlinearities gives $\epsilon_{2} \approx \epsilon(\omega)$.

We now estimate the maximum indirect contribution to $\vec{P}_{1}$ relative to the direct contribution. For a fixed magnitude of $\vec{P}_{2}$, the indirect two-photon absorption is greatest when $\vec{p}_{2}^{\|}=0$, due to the enhancement factor $(\epsilon(2 \omega)-\epsilon(\omega))^{-1}$. From Eqs. (D.3) and (D.9)

$$
\frac{\left|\vec{P}_{1}^{\text {indirect }}\right|}{\left|\vec{P}_{1}^{\text {direct }}\right|} \leq \frac{4 \pi\left|\chi^{(2)}\right|^{2}}{|\epsilon(2 \omega)-\epsilon(\omega)|\left|\chi_{\text {eff }}^{(3)}\right|} .
$$

In this expression, we have used the facts that by symmetry $\chi_{i j k}^{(2)}(-\omega ;-\omega, 2 \omega)$ is the complex conjugate of $\chi_{k j i}^{(2)}(-2 \omega ; \omega, \omega)$ and that zinc blende materials have only one non-vanishing component of $\chi^{(2)}$, the one in which $i, j$, and $k$ represent three different axes. For linear polarization, and defining $\vec{\varepsilon}_{1}$ as in Eqs. (D.1), one has

$$
\chi_{e f f}^{(3)} \approx \frac{\beta c^{2} n^{2}}{8 \omega} .
$$

In the frequency range of interest in this work, we have $\hbar \omega \approx 2 \mathrm{eV}, n \approx 2.4$, and $\beta \approx 10^{-16} \mathrm{~cm}-\mathrm{sec} / \mathrm{erg}(1 \mathrm{~cm} / \mathrm{GW})$. The dielectric constants may be estimated from ${ }^{5}$

$$
\epsilon \approx 3.02+2.10 /\left(1-(\lambda / 253.2)^{2}\right)
$$


where the wavelength $\lambda$ is in nm. One may estimate $\left|\chi_{2}^{(2)}\right|^{2}$ from Miller's empirical rule $^{6}$

$$
\chi_{i j k}^{(2)}\left(-\omega_{1}, \omega_{2}, \omega_{3}\right)=\chi_{i i^{\prime}}^{(1)}\left(\omega_{1}\right) \chi_{j j^{\prime}}^{(1)}\left(\omega_{2}\right) \chi_{k k^{\prime}}^{(1)}\left(\omega_{3}\right) \Delta_{i^{\prime} j^{\prime} k^{\prime}}
$$

The term $\Delta_{i j k}$ is approximately independent of frequency. Levine reports that $\Delta_{i j k} \sim 10^{-6}$ esu for $\mathrm{ZnS}$ (wurtzite) ${ }^{6}$. Because of a factor of 2 difference in his definition of $\vec{\varepsilon}_{1}$ and the definition used here, Levine's $\Delta_{i j k}$ must be scaled up by a factor of 4 in this work. (It is expected that this value will be little changed in the zinc blende structure of $\mathrm{ZnS}$, since both wurtzite and zinc blende are tetrahedrally coordinated and since Levine calculated $\Delta_{i j k}$ using only nearest-neighbor considerations.) Using $\chi^{(1)}=(\epsilon-1) / 4 \pi$, the ratio of interest is estimated to be small.

$$
\frac{\left|\vec{P}^{\text {indirect }}\right|}{\left|\vec{P}^{\text {direct }}\right|} \approx 0.01
$$

Therefore, indirect two-photon absorption is assumed negligible in $\mathrm{ZnS}$ over the range $E_{G} \leq 2 \hbar \omega \leq 2 E_{G}$. The reason it is small is that $\epsilon(2 \omega)-\epsilon(\omega)$ is of the order of unity due to dispersion - i.e., no significant enhancement of $\vec{P}_{2}^{\perp}$ occurs in Eqs. (D.9). Owing to the smallness of $\left|\chi^{(2)}\right|^{2} /\left|\chi^{(3)}\right|$, enhancement is necussary if indirect two-photon absorption is to be comparable to direct two-photon absorption in $\mathbf{Z n S}$.

\section{References}

1. E. Yablonovitch, C. Flytzanis, and N. Bloembergen, "Anisotropic interference of three-wave and double two-wave frequency mixing in GaAs," Phys. Rev. Lett. 29(13), 865-8, (25 Sept. 1972).

2. A. Yariv, "Optical second harmonic generation," in Quantum Electronics, Second Edition, 424 (J. Wiley and Sons, N. Y., 1975). 
3. N. Bloembergen and P. S. Pershan, "Light waves at the boundary of nonlinear media," Phys. Rev. 128, 606-22 (1962).

4. P. N. Butcher, Nonlinear Optical Phenomena, Bulletin 200, Engineering Experiment Station (Ohio State University, 1965).

5. Gmelins Handbuch der Anorganische Chemie, Zink, System-Nummer 32 (Verlag Chemie, G. m. B. H., Weinheim, 1956).

6. B. F. Levine, "Bond-charge calculation of nonlinear optical susceptibilities for various crystal structures," Phys. Rev. B 7(6), 2600-26 (15 Mar. 1976). 
Appendix E

\section{BOUNDARY CONDITIONS ON THE THERMAL LENS}

The effect of surface heat loss was neglected in Chapters 4 and 5 . We now shall show that surface radiation and free convection into air are indeed insignificant in comparison to thermal conduction in the solids of interest. The latter quantity is of the order of $k_{t h} / w$, or $1 \mathrm{~W} \mathrm{~cm}^{-2} \mathrm{~K}^{-1}$ for a beam size of $0.01 \mathrm{~cm}$ in a typical glass.

Radiative transfer in thermal equilibrium obeys a Stefan-Boltzmann type of relation.

$$
\phi_{R a d}=\epsilon \sigma T^{4}
$$

where $\epsilon$ is a dimensionless total emissivity relative to a black body $(0<\epsilon<1), \sigma$ is the Stefan-Boltzmann constant and $\phi_{R a d}$ is a total radiative flux. An imbalance in radiative flux occurs for small, temporary differences in temperature between two adjoining volumes. The net transport from the hotter volume to the cooler is $\triangle \phi_{\text {Rad }}$, where

$$
\triangle \phi_{\text {Rad }}=\epsilon \sigma\left(T_{H o t}^{4}-T_{C o o l}^{4}\right) \leq 4\left(\sigma T^{4}\right)\left(\frac{\triangle T}{T}\right) .
$$

At room temperature, $\sigma T^{4}$ is about 0.05 , and in thermal lensing, $\triangle T / T$ is about 0.001. Therefore $k_{t h} \Delta T \gg \Delta \phi_{R a d}$, since

$$
\frac{\triangle \phi_{R a d}}{\Delta T} \leq 2 \times 10^{-4} \mathrm{~W} \mathrm{~cm}^{-2} \mathrm{~K}^{-1}
$$

Free convection to air contributes comparably. It occurs when the air abutting the thermal lens cyclically heats, expands, rises because of its decrease in density, and is replaced by cooler air from below. Empirically, free-convection losses $\Delta \phi_{C o n v}$ are modeled by Newton's relation ${ }^{1}$

$$
\Delta \phi_{\text {Conv }}=h \triangle T
$$

where $h$ is the average coefficient of free-convection. For air at room temperature, 
standard engineering texts give empirical values of $h$ in the range of $3 \mathrm{~mW} \mathrm{~cm}^{-2} \mathrm{~K}^{-1}$ or less. ${ }^{1}$

$$
\frac{\triangle \phi_{C o n v}}{\Delta T}<3 \times 10^{-3} \mathrm{~W} \mathrm{~cm}^{-2} \mathrm{~K}^{-1} .
$$

The reason for the low convective loss is due to a thermally insulating boundary layer of stagnant air which exists next to an air-solid interface.

Since heat transport across the surface of the thermal lens is negligible, the surface boundary conditions on the temperature are therefore

$$
\left.\frac{\partial T}{\partial z}\right|_{z=0}=\left.\frac{\partial T}{\partial z}\right|_{z=\ell}=0,
$$

in accordance with our assumption in Chapters 4 and 5.

\section{Reference}

F. Kreith, Principles of Heat Transfer, (International Textbook Co., Scranton, Pennsylvania, 1963). 
Appendix F

\section{THERMAL LENSING BIBLIOGRAPHY}

In the past few years, the area of thermal lensing has received much attention. It is difficult to cite every work that has appeared on the subject. The author offers his regrets to any colleague whose work he has not mentioned in the following bibliography. For the sake of limiting the list, certain closely related fields such as

photoacoustics are regarded as separate topics and, by and large, are not included in the bibliography. The list covers the period from Jan. 1964 to approximately Jan. 1984 . 


\section{A Photothermial Lensing Bibliography January 1964 to Approximately January 1984}

1) L. C. Aamodt and J. C. Murphy, "Effect of 3-D heat flow near eiges in photothermal measurements," Appl. Opt. 21 (1) 111-15 (1982).

2) L. C. Aamodt and J. C. Murphy, "Photothermal measurements using a localized excitation source," J. Appl. Phys. $\underline{52}(8)$, 4903-14 (1981).

3) L. C. Aamodt and J. C. Murphy, "Thermal effects in phototherma 1 spectroscopy and photothermal imaging," J. Appl. Phys. 54 (2), 581-91 (1983).

4) S. A. Akhmanov, D. P. Krindach, A. V. Migulin, A. P. Sukhorukov, and R. V. Khokhlov. "Thermal self-actions of laser beams," IEEE J. Quantum Electron 4 (10), 568 (October 1968).

5) N. Alyassini and J. H. Parks, "Time resolved study of laser-induced structural damage in thin films," Laser Induced Damage in Optical Materials: 1975, NBS Special Publication 435, U. S. Dept. of Commerce, National Bureau of Standards, p. 284.

6) N. Alyassini and J. H. Parks, "Measurement of free electron density at the onset of laser-induced surface damage in BSC-2," Laser Induced Damage in Optical Materials: 1975, NBS Special Publication 435, U. S. Dept. of Commerce, National Bureau of Standards, p. 356.

7) N. Alyassini, J. H. Parks and L. G. deShazer, "Time resolution of laser-induced damage to thin films," Laser Induced Damage in Optical Materials: 1973, NBS Special Publication 387, U. S. Dept. of Commerce, National Bureau of Standards, p. 133. 
8) N. Amer and M. A. Olmstead, "A novel method for the study of optical properties of surfaces," Surface Science 132, 68-72 (North Holland Publishing Co., Amsterdam, 1983).

9) N. M. Amer, "In situ ultratrace gas detection by photothermal spectroscopy: An overview," Springer Ser. Opt. Sci. 39 . 299-302 (1983).

10) N. M. Amer, A. Skumanich, and W. B. Jackson, "The contribution of the Staebler-Wronski effect to gap-state absorption in hydrogenated amorphous silicon," Physica B and C Part 2 (Netherlands) 117-118, 897-8 (March 1983).

11) N. M. Amer, A. Skumanich, and W. B. Jackson, "The effects of compensation on light-induced metastable defects in hydrogenated amorphous silicon," J. Non-Cryst. Solids 59-60 (1), 409-12 (1983).

12) G. A. Askar'yan and I. L. Chisty, "Thermal self-focusing in a light beam with low intensity near the axis," Zh. Eksp. Teor. Fix. 58(1), 133-4 (1970).

13) J. Badoz and D. Fournier, editors, "Photoacoustic and photothermal spectroscopy," (Proceedings of the 3rd International Topical Meeting; 5-8 Apri1, 1983; Paris, France), J. Phys., Colloq. 44 (C6), (October 1983).

14) R. T. Bailey, F. R. Cruickshank, R. Guthrie, D. Pugh and I. J. M. Weir, "Extraction of energy transfer rate constants from the pulsed laser generated thermal lens spectrometer signal," J. Mol. Struct. 80, 433-6 (1982). 
15) R. T. Bailey, F. R. Cruickshank, R. Guthrie, D. Pugh and I.J.M. Weir, "Vibrational energy transfer rate constants from the thermal lens," Proc. Int. Conf. on Time-Resolved Vib. Spectrosc. (1982), George H. Atkinson, editor, 121-5 (Academic Press, N.Y., 1983).

16) R. T. Bailey, F. R. Cruickshank, D. Pugh, A. McLeod, and W. Johnstone, "Gas-phase thermal diffusivities by a thermal lens technique," Chem. Phys. 68 (3), 351-7, (1982).

17) R. T. Bailey, F. R. Cruickshank, D. Pugh, and W. Johnson, "Pulsed-source thermal lens. Part 1. Theoretical Analysis," J. Chem. Soc. Faraday Trans. 2 76 (6), 633-47 (1980).

18) R. T. Bailey, F. R. Cruickshank, D. Pugh, and W. Johnstone, "Pulsed-source thermal lens. Part 2 Experimental tests of the

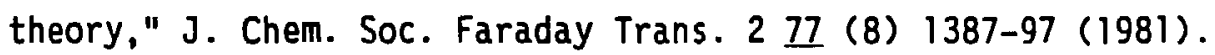

19) R. T. Bailey, F. R. Cruickshank, D. Pugh, S. Guthrie, A. McLeod, W. S. Foulds, W. R. Lee, and S. Venkatesh, "The determination of thermal conductivity coefficients of liquids by a thermal lens technique," Chem. Phys. (Netherlands) $\underline{77}$ (2), 243-6 (1 June 1983).

20) R. T. Bailey, F. R. Cruickshank, W. Johnstone, and D. Pugh, "The sensitivity of the pulsed laser thermal lens spectrometer," J. Mol. Structure 60, 25-9 (1980).

21) R. T. Bailey, F. R. Cruickshank, R. Guthrie, D. Pugh and I. J: M. Weir, "Short time-scale effects in the pulsed source thermal lens,".-Mol. Phys. (Great Britain) 48 (1), 81-95 (Jan. 1983). 
22) R. T. Bailey, F. R. Cruickshank, D. Pugh and W. Johnstone, "The application of the thermal lens to infrared photochemistry," Proc. Conf., Lasers Chem., Michael A. West, editor, 257-64 (Elsevier, Amsterdam, 1977).

23) R. T. Bailey, F. R. Cruickshank, D. Pugh, and W. Johnstone, "The time resolved geometrical optics of the thermal lens," Chem. Phys. Lett. 59 (2) 324-9 (1978).

24) R. T. Bailey, F. R. Cruickshank, D. Pugh, R. Guthrie, W. Johnstone, J. Mayer, and K. Middleton, "Thermal effects in infrared laser photochemistry and energy transfer experiments," J. Chem. Phys. 77 (7), 3453-7 (1 Oct. 19̇82).

25) R. T. Bailey and F. R. Cruickshank, "Thermal lens detection in Raman gain spectroscopy," C. R. - 7th Conf. Int. Spectrosc. Raman, W. F. Murphy, editor, 698-9 (NRCC Canada, 1980).

26). J. S. Bakos and I. B. Foldes, "Self-focusing in laser-produced spark," Hungarian Acad. Sci., Budapest, Hungary, Report KFKI-1983-50 (In English).

27) P. Balkevicius, I. Tulbinas, E. Maldutis and J. Rekonis, "Change in the refractive index during thermal self-focusing of a laser beam in media with negative $\mathrm{dn} / \mathrm{dT}$," Sb. Ref. Vses. Konf "Fiz. Dielektr. Perspekt. Ec Razvit," S. N. Koikov and G. B. Semushkin, editors, Vol. 2, 299-300 (in Russian) (1974) Mtg. date 1973.

28) A. J. Bard, "Photoacoustic and photothermic/spectroscopy," Analusis 6 (7), (in French) 277-83 (1978).

29) H. E. Bass and H. J. Bauer, "Kinetic model for thermal blooming in the atmoshpere," Appl. Opt. 12 (7), 1506-10 (1973). 
30) Michael Bass, Eric W. Van Stryland and A. F. Stewart, "Laser calorimetric measurement of two-photon absorption," Appl. Phys. Lett. 34 (2), 142, (15 January 1979).

31) T. Baumann, F. Dacol, and R. L. Meicher, "Transmission thermal wave microscopy with pyroelectric detection," Appl. Phys. Lett. 43 (1), 71-3 (1 July 1983).

32) V. N. Bel'tyugov, I. I. Sukhanov, and Yu V. Troitskii, "Thermal distortions of intercavity optical components of a laser during surface absorption of radiation," Autometriya 2 . 115-24 (in Russian) (1977).

33) I. Sh. Beluga, G. S. Vinevich, and L. A. Kilosovskaya, "Disturtion of a Gaussian beam by a thermal lens formed in optical elements," Opt. Spectrosc. (USSR) 50 (3), 292-4 (March 1981).

34) B. Bendow, P. D. Gianino, S. S. Mitra and Yet-ful Tsay, "Stress and pressure dependence of the refractive index of laser window materials," Third Conference on High Power Infrared Laser Window Materials, November 12-14, 1973. AFCRL-TR-74-0085(I), C. A. Pithow and B. Bendow, editors, 367 (Air Force Cambridge Research Laboratory, L. G. Hanscom Field, Bedford, Massachusetts 01730, 14 Feb. 1974).

35) B. Bendow, L. H. Skolkik, and P. D. Gianino, "A review of research on thermal lensing from laser window materials," Conference on High Power Infrared Laser Window Materials, October 30,31 and November 1, 1972, AFCRL-TR-73-0372(I), C. A. Pitha, editor, 39 (Air Force Cambridge Research Laboratories, L. G. Hanscom Field, Bedford, MA 01730, 19 June 1973). 
36) Paul J. Berger, Peter B. Ullrich, Jeanne T. Ullrich and Frederick G. Gebhardt, "Transient thermal blooming of a slewed laser beam containing a region of stagnant absorber," Appl. Opt. 16 (2), 345-54 (1977).

37) Enrique Bernal G., "Heat flow analysis of laser absorption calorimetry," Appl. Opt. 14 (2), 314-21 (February 1975).

38) S. E. Bialkowski, "The effects of mass diffusion in gas-phase thermal lens experiments," Chem. Phys. Lett. 104 (5), 448-54 (1984).

39) A. C. Boccara, D. Fournier, Warren Jackson, and Nabil M. Amer, "Sensitive photothermal deflection technique for measuring absorption in optically thin media," Opt. Lett. $\underline{6}$ (9), 377-9 (September 1980).

40) A. C. Boccara, D. Fournier, Warren Jackson, and Nabil M. Amer, "Erratum: Sensitive photothermal deflection technique for measuring absorption in optically thin media," Opt. Lett. $\underline{6}$ (1), 51 (January 1981).

41) Yu. I. Boiko and A. A. Libenson, "Thermal self-focusing of laser radiation in alkali halide single crystals," Fix. Tverd. Tela 13(2), 656-8 (1971) (In Russian).

42) J. H. Brannon and D. Magde, "Absolute fluorescence yields by thermal blooming: fluorscein and cresyl violet," Springer Series Chem. Phys. 3 Adv. Laser Chem. 1292-6 (1978).

43) James H. Brannon and Douglas Magde, "Absolute quantum yield determination by thermal blooming Fluorescein," Phys. Chem. $\underline{82}$ (6), 705-9 (23 March 1978). 
44) George H. Brilmyer and Allen J. Bard, "Application of photothermal spectroscopy to in-situ studies of films on metals and electrodes," Anal. Chem. $\underline{52}$ (4), 685-91 (1980).

45) George H. Brilmyer, Akira Fujishima, K. S. V. Santhanam and Allen J. Bard," Photothermal spectroscopy," Anal. Chem. $\underline{49}$ (13), 2057-62 (1977).

46) George Howard Brilmyer, "Photothermal spectroscopy fundamentals and applications," PhD. Dissertation U. T. Austin, TX, Univ. Microfilms, Ann Arbor, MI, Order No. 7928265 (1979).

47) Robert T. Brown and David C. Smith, "Aerosol-induced thermal blooming," J. Appl. Phys. 46 (1), 402-5 (1975).

48) V.V. Bryskin and L. I. Korovhin, "Nonlinear theory of electric field distribution dynamics in photorefractive crystals," Fiz. Tverd. Tela $25(1), 55$ (Jan. 1983) (In Russian).

49) S. R. J. Brueck, H. Kildal, and L. J. Belanger, "Photoacoustic and photorefractive detection of small absorptions in liquids," Opt. Commun. 34 (2), 199-204 (August 1980).

50) M. M. Bubov, I. M. Buzhinski i, E. M. Dianov, S. K. Mamonov, L. I. Mikhailova and A. M. Prokhorov, "Change in the sign of a glass laser rod thermal lens during a change in the thermoptical constant of glass," Dok' Akad Nauk. SSSR $\underline{205}$ (3), 556-9 (1972) (In Russian).

51) C. E. Buffett and M. D. Morris, "Convective effects in thermal lens spectroscopy," App. Spectrosc. 37 (5), 455-8 (Sept.-Oct. 1983). 
52) C. E. Buffett and M. D. Morris, Microcell thermal lens detector for liquid chromatography," Anal. Chem. 55 (2), 376-8 (1983).

53) C. E. Buffett and M. D. Morris, "Thermal lens detection for liquid chromatography," Anal. Chem. 54 (11), 1824-5 (1982).

54) Mitchell S. Burberry, James A. Morrel1, A. C. Albrecht, and Robert L. Swofford, "Local mode overtone intensities of $\mathrm{C}-\mathrm{H}$ stretching modes in alkanes and methyl substituted benzenes," J. Chem. Phys. 70 (12), 5522 (15 June 1979).

55) A. Burgos, H. Mancini, E. Quel, J. Wesfried, "Optimum laser cavity and internal cell place for thermal lens effect measurements by the photomixing method," Opt. Commun. $\underline{20}$ (3), 343-7 (1977).

56) Rudolf G. Buser and Robert S. Rhode, "Transient thermal blooming of long laser pulses," Appl. Opt. 14 (1), 50-5 (1975).

57) Gerd Busse, "Optoacoustic and photothermal material inspection techniques," Appl. Opt. 21 (1), 107-10 (1982).

58) G. Busse, "Photothermal transmission probing of a metal," Infrared Phys. 20 (6), 419-22 (1980).

59 G. Busse and P. Eyerer, "Thermal wave remote and nondestructive inspection of polymers," Appl. Phys. Lett. $\underline{43}$ (4), 355-7 (15 Aug. 1983).

60) W. R. Callen, B. G. Huth, and R. H. Pantell, "Optical patterns of thermally self-defocused light," Appl. Phys. Lett. 11 (4), 103 (1 August 1967). 
61) P. Calmettes and C. Laj, "Simultaneous measurements of the thermal diffusion coefficient and of the thermal conductivity of transparent media by means of a thermal lens effect," $\mathrm{J}$. Phys. (Paris) 33 (2-3) Suppl. pp. 125-9 (1972) (In English).

62) P. Calmettes and C. Laj, "Study of heat diffusion in transparent media by means of a thermal lens effect," Phys. Rev. Lett. 27 (5), 239-42 (2 August 1971)

63) A. J. Campillo, C. J. Dodge and H.B. Lin, "Aerosol particle absorption spectroscopy by photothermal modulation of Mie scattered light," Appl. Opt. 20 (18), 3100-3 (1981).

64) A. J. Campillo, S. J. Petuchowski, C. Christopher, and H. B. Lin, "Fabry-Perot photothermal trace detection," Appl. Phys. Lett. 41 (4), 327-9 (1982).

65) Anthony J. Campillo and Horn Bond Lin, "Photothermal spectroscopy of aerosols," Proc. SPIE-Int. Soc. Opt. Eng. 286, Laser Spectrose., 24-32 (1981).

66) R. L. Carman and P. L. Kelley, "Time dependence in the thermal blooming of laser beams," Appl. Phys. Lett. 12 (8), 241 (15 April 1968).

67) C. A. Carter, "Development and characterization of thermal lens calorimetry for chemical analysis," Ph.D. Dissertation, Univ. Utah, Salt Lake Sity, UT, Univer. Microfilms, Ann Arbor, MI, Order \#DA8312297.

68) C. A. Carter, J. M. Brady and J. M. Harris, "Infrared laser-induced thermal lens calorimetry," Appl. Spectrosc. $\underline{36}$ (3), 309-14 (1982). 
69) C. A. Carter and J. M. Harris, "Comparison of models describing the thermal lens effect," Appl. Opt. $\underline{23}$ (3), 476-81 (1984).

70) C. A. Carter and J. M. Harris, "Comparison of single- and dual-beam configurations for thermal lens calorimetry, Anal. Chem. 55 (8), 1256-61 (1983).

71) C. A. Carter and J. M. Harris, "Lony path length samples in thermal lens calorimetry," Appl. Spectrosc. 37, 166-72 (Feb. 1983).

72) C. A. Carter and J. M. Harris, "Thermal lens absorption measurements on small volume samples," Anal. Chem. 56 (6), 922-5 (1984).

73) D. Chardon and S. J. Huard, "Abso:ption losses and triermal diffusivity of optical fibers investigated by photothermal method: Theory of experiments," Can. J. Phys. 61 (9), 1334-46 (Sept. 1983).

74) Tsuey Ing Chen and M. D. Morris, "Photothermal deflection densitometer for thin-layer chromatography," Anal. Chem. 56 (1), 19-21 (1984).

75) H. Coufal, "Photothermal spectroscopy using a pyroelectric thin film detector," Report TR-5 (1983). Avail. NTIS order \#AD-A134291.

76) David A. Cremers and Richard A. Keller, "Thermally induced laser pulsing to measure weak optical absorptions," Appl. Opt. 20 (22), 3838-48 (15 November 1981). 
77) David A. Cremers and Richard A. Keller, "Thermooptic-based differential measurements of weak solute absorptions with an interferometer," App1. Opt. $\underline{21}$ (9), 1654-62 (1 May 1982).

78) F. W. Dabby, T. K. Gustafson, J. R. Whinnery, Y. Kohanzadeh, and P. L. Kelley, "Thermally self-induced phase modulation of laser beams," Appl. Phys. Lett. 16 (9), 362 (1 May 1970).

79) K. Daree, "Photochemical blooming of laser beams," Opt. Commun. 4 (3), 238 (November 1971).

80) H. W. Deckman, C. B. Rox10, and E. Yablontovitch, "Maximum statistical increase of optical absorption in textured semiconductor films," Opt. Lett. $\underline{8}$ (9), 491-3 (Sept. 1983).

81) J. Doria, M. R. Heras, A. Tovar and J. Yuguero, "Calorimetric system for the study of photothermal selectivity," An. Fis. Ser. B 78 (1), 83-8 (1982) (In Spanish).

82) N. J. Dovichi and J. M. Harris, "Differential thermal lens calorimetry," Ana1. Chem. $\underline{52}$ (14), 2338-42 (December 1980).

83) N. J. Dovichi and J. M. Harris, "Frequency response of the thermal lens," Proc. SPIE Int. Soc. Opt. Eng. 288, 372-5 (1982) Los Alamos Conf. on Optícs '81 (7-10 April 1981 Los Alamos, N. M.).

84) N. J. Dovichi and J. M. Harris, "Laser induced thermal lens effect for calorimetric trace analysis," Anal. Chem. 디 (6), 728 (May 1979). 
85) N. J. Dovichi and J. M. Harris, "Thermal lens calorimetry for flowing samples," Anal. Chem. 53 (4), 689-92 (1981).

86) N. J. Dovichi and J. M. Harris, "Laser induced thermal lens effect for calorimetric trace analysis," Anal. Chem. 51 (6), 728-31 (1979).

87) N. J. Dovichi and J. M. Harris, "Time-resolved thermal lens calorimetry," Anal. Chem. $\underline{53}$ (1), 106-9 (January 1981).

88) R. C. DuVarney, A. K. Garrison and G. Busse, "Electron paramagnetic resonance spectroscopy with photothermal and cotoacoustic detection," Appl. Phys. Lett. 38 (9), 675-6 (1981).

89) P. Eyerer and G. Busse, "Thermal wave analysis of polymers using photothermal deflection," Kunststoffe 73 (9), 547-9 (1983).

90) Howard L. Fang and Robert L. Swofford, "Analysis of the thermal lensing effect for an optically thick sample - A revised mode1," J. Appl. Phys. 50 (11) Part 1, 6609-15 (1979).

91) Howard L. Fang and Robert L. Swofford, "The thermal lens in absorption spectroscopy," in Ultrasensitive laser spectroscopy, David S. Kliger, editor (Academic Press, N.Y., 1983).

92) Howard L. Fang, T. L. Gustafson, and Robert L. Swofford, "Two-photon absorption photothermal spectroscopy using a synchroriously pumped picosecond dye laser. Thermal lensing spectra of naphthalene and diphenylbutadiene," J. Chem. Phvs. 78 (4), 1663-9 (1983). 
93) H. L. Fang, T. L. Gustafson, and R. L. Swofford, "Two-photon thermal lensing spectroscopy using a synchronously pumped dye laser," CLEO ' 82 , postdeadline paper.

94) A. I. Ferguson and C. N. Ironside, "Picosecond dichroism detected by photothermal deflection," Proc. SPIE 369, Max Born Centennial Conf., 1982, 374-8 (1983).

95) M. R. Fisher and N. S. Nogar, "Thermal spectroscopy," J. Chem. Educ. 59 (1), 15-6 (1982).

96) J. D. Foster and L.M. Osterink, "Thermal effects in a Nd:YAG laser," J. Appl. Phys. 4l(9), 3656-63 (1970).

97) D. Fournier, A. C. Boccara and J. Badoz, "Photothermal deflection Fourier transform spectroscopy: a tool for high-sensitivity absorption and dichroism measurements," Appl. Opt. 21 (1), 74-6 (1981).

98) D. Fournier, A. C. Boccara, Nabil M. Amer and Robert Gerlach, "Sensitive in situ trace gas detection by photothermal deflection spectroscopy," Appl. Phys. Lett. $\underline{37}$ (6), 519-21 (1980).

99) D. Fournier, A. C. Boccarz, F. LePoutre and J. Badoz, "Photoacoustic and photothermal deter.tion for telecommunications," Rev. Phys. App 1. (France) 19 (2), 59-62 (Feb. 1984) (in French).

100) D. M. Freidrich and S. A. Klem, "Excited triplet state polarizabilities in laser induced refractive index tránsients," Chem. Phys. 41 (1), 153-62 (1 August 1979). 
101) Robert A. Frosch, Jovin Moccanin, Amitava Gupta and Su Don Hong (Inventors), "Double beam optical method and apparatus for measuring thermal diffusivity and other molecular dynamic processes," U.S. Pat. 4243327, date: 810106.

102) Y. Fujii, K. Kajimura, S. Ishihara and H. Yujima, "Thermal-gradient-induced optical deflection in titanium dioxide crystals," Appl. Phys. Lett. 41 (3), 217-9 (1 Aug. 1982).

103) Akira Fujishima, Hideki Masuda, Kenichi Honda and Allen J. Bard, "Measurement of gold electron surface changes in situ by laser photothermal spectroscopy," Anal. Chem. 52 (4), 682-5 (1980).

104) Kitao Fujiwara, Kei ichiro Huwa and Takayoshi Kobayashi, "Laser thermal-lens effect and its application to photometric analysis," Kagaku (Kyoto) 36 (6), 432-8 (1981) (In Japanese).

105) Kiyokazu Fuke, Masayasu Ueda and Michiya Itoh, "Thermal lensing study of singlet oxygen reactions," J. Am. Chem. Soc. 105 (5), 1091-6 (1983).

106) N. E. Galich, "Thermal structures initiated by laser radiation in a weakly absorbing medium, and thermal breakthrough during motion of a beam," Zhurnal Tekhnicheskoi Fiziki 53(4), 768 (April 1983) (In Russian).

107) Stanislaw Gebala, "Method for photothermal changes of the refractive index in glasses," Szklo Ceram. 28 (12), 318-9 (1977) (In Pollsh). 
108) Stanislaw Gebala, "On photothermal reduction of the luminescence of cerium crown glasses," Opt. Appl. $\underline{6}$ (2), 65-8 (1976).

109) Frederic G. Gebhardt, David C. Smith, Rudolph G. Buser and Robert $S$. Rohde, "Turbulence effects on thermal blooming," App 1. Opt. 12 (8), 1794-805 (1978).

110) P. Geraghty, "Photothermal spectroscopy of surface adsorbates," Ph.D. Dissertation, U. of Mich. Univ. Microfilms, Int. Order \#DA8314277.

111) M. Giglio and A. Vendramini, "Thermal lens effect in a binary liquid mixture new effect," Appl. Phys. Lett. 25 (10), 555-7 (1974).

112) M. J. Giles, "The thermal self-focusing of a wave beam in an under-dense plasma. I. The wave spectrum," J. Plasma Phys. 29. Part 2 (Great Britain), 325-58 (April 1983).

113) M. J. Giles, "The thermal self-focusing of a wave beam in an underdense plasma. II. Energy transfer to ions," J. Plasma Phys. 29, Part 3 (Great Britain), 449-64 (June 1983).

114) M. J. Giles, "The thermal self-focusing of a wave beam in an underdense plasma. III. Linear convection," J. Plasma Phys. 29, Part 3 (Great Britain), 465-90 (June 1983).

115) M. J. Giles, "The thermal self-focusing of a wave beam in an underdense plasma. IV. Quadratic nonlinear effects," J. Plasma Phys. 29, Part 3 (Great Britain), 491-512 (June 1983). 
116) M. J. Giles, "The thermal self-focusing of a wave beam in an underdense plasma. V. Cubic nonlinear effects," J. Plasma Phys. 29, Part 3 (Great Britain), 513-23 (June 1983).

117) A. J. Glass, "Thermal blooming in gases," Opto-electronics 1 (4), 174-8 (1969).

118) H. J. Goldsmid, M. M. Kaila and G. L. Paul, "Thermal conductivity of amorphous silicon," Phys. Status Solidi A 766(1), K31 (March 1983).

119) V. M. Gordienko, V. G. Kolomiets and P. J. Chernega, "Thermal self-focusing in a proustite crystal and measurement of its thermophysical parameters," Kvantovaya Elektron (Moscow). 1 (12), 2642-4 (1974) (In Russian).

120) J. P. Gordon,. R. C. C. Leite, R. S. Moore, S. P. S. Porto, and J. R. Whinnery, "Long-transient effects in lasers with inserted liquid samples," 3. Appl. Phys. 36 (1), 3-8 (January 1965).

121) G. Gouesbet, M. Rhazi and M. E. Wei11, "New heartbeat phenomenon, and the concept of 2-D optical turbulence," Appl. Opt. 22, 304-9 (15 Jan. 1983).

122) V. I. Grishko, I. G. Udelevich, V. P. Nikitina, "Theoretical aspects and applications of thermal lens calorimetry -- a new tool," Zh. ńnal. Khim. 39 (1), 129-41 (1984) (In Russian).

123) Mool C. Gupta, Su-Don Hong, Amitava Gupta and Jovan Moccanin, "Thermal diffusivity measurements using a pulsed dual-beam thermal lens technique," Appl. Phys. Lett. 37(6), 505-7 (15 Sept. 1980). 
124) V. L. Gurevich and A. K. Tagantsev, "Dielectric relaxation and thermal polarization effect," Izvest. Akad. Nauk SSSR Fiz. (Bull. Acad. Sci. USSR, Phys.) 47(3), 533 (1983). Proc. of 2nd all-union seminar on physics of ferroelastics--Voronezh, 1-3 Apri1 1982.

125) K. Handrich, "Phonon lifetime and thermal conductivity in amorphous solids," Thin Solid Films 102(1), 533 (8 April 1983).

126) J. M. Harris and N. J. Dovichi, "Thermal lens calorimetry," Ana 1. Chem. 52 (6), 695A-706A (6 May 1980).

127) R. I. Harris and M. E. Gangl, "Thermo-optic coefficient $(\partial \mathrm{n} / \partial \mathrm{T})$ of $1.3 \mu \mathrm{m}$ laser window materials," NBS Spec.

Pub1. 638, 171-4 (1984).

128) Jeanne P. Haushalter and Michael D. Morris, "Use of thermal blooming spectroscopy for enzymic anaiyses," App1. Spectrosc. 34 (4), 445-7 (1980).

129) Phillip D. Henshaw, "Laser beamsteering using the photorefractive effect," Appl. Opt. 21 (13), 2323-5 (1 July 1982).

130) J. P. Herriau, J. P. Huignard, and L. Jounhomme, "Light beam steering using a reversible photoinduced grating in BSO crystals." European Conference on optical communications, 21-24 Sept. 1982, Cannes-Frances, 439-41 (1982).

131) T. Higashi, T. Imasaka, and N. Ishibashi, "Thermal lens spectrophotometry with argon laser excitation source for nitrogen dioxide determination," Anal. Chem. 55 (12), 1907-10 (Oct. 1983). 
132) Charles B. Hogge, "Thermal blooming of high-energy laser beams," Proc. Int. Conf. Lasers (1978), 633-6 (1979).

133) C. B. Hogge, "Aberrations in high power laser systems," Proc. SPIE 293, Conference on Wavefront Distortions in High Power Optics, held in San Diego, CA, 20 July 1981.

134) A. Hordvik, "An experimental investigation of thermal distortion in infrared window materials," Conf. on High Power Infrared Laser Window Materials, 27-28 October 1971 , AFCRL-TR-71-0592, Charles S. Sahagian and Carl A. Pitha, editors, 389 (Air Force Carabridge Research Laboratories, L. G. Hanscom Field, Bedford, Massachusetts 01730, 13 December, 1971).

135) A. Hordvik, "Measurement techniques for small absorption coefficients: Recent advances," Appl. Opt. 16 (11), 2827-33 (November 1977).

136) Chenming Hu and J. R. Whinnery, "New thermooptical measurement method and a comparison with other methods," Appl. Opt. 12 (1), 72-9 (January 1973).

137) Robert J. Hull, P. L. Kelly, and R. L. Carman, "Self-induced thermal lens effect in carbon tetrachloride in the presence of beam motion," Appl. Phys. Lett. 17(12), 539-41 (1971).

138) Totaro Imasaka and Nobuhiko Ishibashi, "Thermal lens absorptiometric analysis," Kagaku (Kyoto) 37 (2), 161-3 (1982) (In Japanese). 
139) Totaro Imasaka and Nobuhiko Ishibashi, "Thermal lens spectroscopy," Tr. AC Trends Anal. Chem. (Pers. Ed.) Vol. 1 (12), 273-7 (1982).

140) Totaro Imasaka and Nobuhiko Ishibashi, "Thermal lens spectroscopy," Reza Kenkyo 11 (2), 129-37 (1983) (in Japanese).

141) Totaro Imasaka and Katsuhiro Shimanoe, "Thermal lens spectroscopic studies of radiationless processes in monemeric nitrogen dioxide," J. Chem. Phys. 79 (7), 3201-5 (1 Oct. 1983).

142) Totaro Imasaka, Kazuhiko Miyaishi and Nobuhiko Isiibashi, "Application of the thermal lens effect for determination of iron (II) with 4,7-diphenyl-1,10-phenanthroline disulfonic acid," Anal. Chim. Acta 115, 407-10 (1980).

143) Masahide Itoh and Iwao Ogura, "Absorption measurements of laser optical materials by interferometric calorimetry," $\mathrm{J}$. App 1. Phys. 53 (7), 5140-5 (July 1982).

144) N. R. Ivanov and L. A. Shuvalov, "Thermo-optic and electro-optic properties of tri hydrogen selenites," J. Phys. Soc. Jap. Supp1. 28, 72-4 (1970).

145) W. B. Jackson and N. M. Amer., "Subgap and band edge optical absorption in hydrogenated amorphous silicon by photothermal deflection spectroscopy," J. Phys. Colloq. C4 Part 1, 293-6 (1981).

146) W. B. Jackson, D. K. Biegelsen, R. J. Nemanich and J. C. Knights, "Optical absorption spectra of surface or interface states in hydrogenated amorphous silicon," Appl. Phys. Lett. 42 (11), 105-7 (1 Jan. 1983). 
147) W. B. Jackson, N. M. Amer, A. C. Boccara, and D. Fournier, "Photothermal deflection spectroscopy and detection," Appl. Opt. 20 (8), 1333-44 (15 Apri1 1981).

148) W. B. Jackson, N. M. Johnson, and D. K. Biegelsen, "Density of gap states of silicon grain boundaries determined by optical absorption," Appl. Phys. Lett. 43 (2), 195-7 (15 July 1983).

149) W. B. Jackson and R. J. Nemanich, "The absolute luminescence quantum efficiency in hydrogenated amorphous silicon," J. Non-Cryst. Solids 59-60 (1), 353-6 (1983).

150) W. B. Jackson, R. J. Nemanich, and N. M. Amer, "Energy dependence of the carrier mobility-lifetime production in hydrogenated amorphous silicon," Phys. Rev. B 27 (8), 4861-71 (15 Apr. 1983).

151) Hou Jing-ya, "Evaluation of the thermal focal length produced in a repetitively pulsed solid-state laser," J. Appl. Phys. $\underline{54}$ (5), 2214 (May 1983).

152) A. T. Jones and J. A. McMordie, "Thermal blooming of continuous wave laser radiation," J. Phys. D 14 (2), 163-72 (1981).

153) Svein Otto Kanstad and Per Erik Nordal, "Photoacoustic and photo-thermal spectroscopy," Phys. Technol. 11 (4), 142-7 (1980).

154) Svein Otto Kanstad and Per Erik Nordal, "Photoacoustic and photo-thermal spectroscopy - Novel tools for the analysis of particulate matter," Powder Technol. 22 (1), 133-7 (1979). 
155) Svein Otto Kanstad and Per Erik Nordal, "Photoacoustic and photo-thermal techniques for powder and surface spectroscopy," Appl. Surf. Sci. 6 (3-4), 372-91 (1980).

156) Svein Otto Kanstad and Per Erik Nordal, "Spectroscopy by photothermal radiometry," SPIE Proc. of the Max Born Centenary Conf. 82, 7-10 Sept. 1982, Edinburgh, Scotland.

157) Joseph $W$. Kantorski, inventor, "Lens balanced laser rod," U.S. patent 3700423, date: 721024 , Nd-glass lens-balanced for elimination of thermal effects.

158) N. V. Karlov, G. P. Kuz'min, and A. M. Prokhorov, "Thermal self-focusing and breakdown of $\mathrm{KCl}, \mathrm{KBr}$, and $\mathrm{CeI}$ under the action of a $\mathrm{CO}_{2}$ laser," Pis'ma Zh. Eksp. Teor. Fiz. 12(7), 363-6 (1970) (In Russian).

159) N. E. Kask, Y. V. Radchenko, G. M. Fedorov, and D. B. Chopornyak, "Temperature dependence of the absorptioni coefficient of optical glasses exposed to laser radiation," Sov. J. Quantum Electron. 9 (2), 193-8 (February 1979).

160) Seymour Paul Keller and Gabor A. Somorjai (inventors), "Photothermal process," Patent: FRANCE 1434842, date: 640601.

161) J. R. Kenemuth, C. B. Hogge, Peter V. Avizonis, "Thermal blooming of a $10.6 \mu$ laser beam in $\mathrm{CO}_{2}$," Appl. Phys. Lett. 17(5), 220-3 (1970).

162) Herbert Kleiman and Richard W. O'Neil, "Deflection of a carbon dioxide laser beam in an absorbing gas," J. Opt. Soc. Amer. 6l(1), 12-15 (1971). 
163) C. Klein, "Thermal lensing in (111)-oriented $\mathrm{KCl}$ windows," in Proceedings of the High Power Laser Optical Components and Component Materials Meeting. Sponsored by Defense Advanced Research Projects Agency, 340 (DARPA 1400 Wilson Blvd., Arlington, VA., 1977).

164) David S. Kliger, "Thermal lensing: A new spectroscopic tool," Accts. Chem. Research 13 (5), 129-34 (May 1980).

165) Y. Kohanzedeh and D. H. Auston, "Measurement of low-absorption coefficients using the beat frequency shift between transverse modes of a laser," IEEE J. Quantum Electron. 6 , 475-7 (1970).

166) Y. Kohanzedeh, Kwing Wai Ma, and J. R. Whinnery, "Measurement of refractive index change with temperature using thermal self-phase modulation," App1. Opt. 12(7), 1584-7 (1973).

167) G. Koren, "Thermal blooming measurement of specific heat and thermal conductivity in isotropic p-methoxybenzylidene-pbutylaniline (MBBA) near clearing," Phys. Rev. A 13 (3), $1177-84$ (1976).

168) D. L. Kwong and Dae M. Kim, "Pulsed laser heating of silicon: The coupling of optical absorption and thermal conduction during irradiation," J. Appl. Phys. 54 (1), 366-73 (January 1983).

169) J. R. Lalanne, E. Seln, J. Buchert and S. Kielich, "Measurements of heat transfer in microemulsions by laser-induced thermal blooming," Appl. Phys. Lett. 36 (12), 973-5 (1980). 
170) R. A. Leach and J. M. Harris, "Thermal lens calorimetry application to chromatographic detection," J. Chromatogr. 218, $15-9$ (1981).

171) R. C. C. Leite, R. S. Moore, and J. R. Whinnery, "Low absorption measurements by means of the thermal lens effect using an He-Ne laser," Appl. Phys. Lett. $\underline{5}$ (7) (1 October 1964).

172) N. I. Lemesh and L. A. Drozdova, "Thermal gas lens in laser gas-discharge tubes," Vopr. Energoperenosa Neodnorodnykh Sredakh, 0. G. Martynenko, P. M. Kolesnikov and I. Vatutin, editors, 131-6 (Akad Nauk B̧̧Ś, Inst. Teplo-Massoobmena, Minsk USSR 1975) (in Russian).

173) F. LePoutre and G. Rousset, "Photothermal measurements of thermal diffusivities," Proc. 7th Int. Heat Transfer Conf., Ulrich Grigul1, E. Hahne, and K. Stephan, editors, Vol. 4, 539-43 (Hemi sphere Pub. Co., Wash. D.C., 1982).

174) Michae1 L. Lesiecki and Micheal J. Drake, "Ușe of the thermal lens technique to measure the luminescent quantum yields of dyes in PMMA for luminescent solar concentrators," App1. Opt. 21 (3), 557-60 (1983).

175) Changjiang Li, "Thermal lens spectroscopy and applications," Wuli 11, 721-3 (1982) (In Chinese).

176) Pedro Lilienfeld, "Monitoring techniques for carbon fiber emissions: Evaluation A," Report EPA 600/2-81-048, Order \#205932 (1981). 
177) Horn Bond Lin and D. U. Fluckiger, "Photothermal detection of nitrogen dioxide," Microbeam Anal. 17, 526-8 (1982).

178) A. G. Litvak, "Self-focusing of powerful light beams by thermal effects," Soviet Physics JETP Lett. 4, 230 (1966).

179) M. E. Long, Robert Swofford, and A. C. Albrecht, "Thermal lens technique: A new method of absorption spectroscopy," Science 19. 183 (16 January 1976).

180) M. J. D. Low, T. H. Arnold, and A. G. Severdia, "The effects of the nature of the coupling gas and its pressure on infrared photothermal beam deflection of solids, "Infrared Physics (Great Britain) 23 (4), 199-206 (July 1983).

181) M. J. D. Low and C. Morterra, "IR studies of carbons I. IR photothermal beam deflection spectroscopy and II. The vacuum pyrolysis of cellulose," Carbon 21 (3), 275-81 (1983).

182) M. J. D. Low and A. G. Severdia, "Infrared spectra of a single human hair," Spectrosc. Lett 16 (11), 871-7 (1983).

183) M. J. D. Low, A. G. Severdia, and T. H. Arnold, "Infrared observation of intrinsic modes on absorbent surfaces by photothermal beam deflection spectroscopy," Spectrosc. Lett. 16. 207-14 (1983).

184) M. J. D. Low and M. Lacroix, "An infrared photothermal beam deflection Fourier transform spectrometer," Infrared Phys. $\underline{22}$ (3), 139-47 (1982). 
185) M. J. D. Low, C. Morterra, A. G. Severdia and M. Lacroix, "Infrared photothermal deflection spectroscopy for the study of surfaces," Appl. Surf. Sci. (Netherlands) 13 (3-4), 429-40 (Sept-Oct 1982).

186) M. J. D. Low, M. Lacroix and C. Morterra, "Infrared photothermal beam deflection Fourier transform spectroscopy of solids," Appl. Spectrosc. $\underline{36}$ (5), 582-4 (1982).

187) M. J. D. LOW, C. Morterra and A. G. Severdia, "Infrared photothermal deflection spectroscopy of carbon-supported metal catalysts," Spectrosc. Lett. 15 (5), 415-21 (1982).

188) M. J. D. Low, M. Lacroix, C. Morterra and A. G. Severdia (Dep. Chem. N.Y.U., NY, NY), "Infrared spectra of solids and large objects without sample preparation," Am. Lab. 14 (6), 16-27 (Fairfield, Conn., 1982).

189) Douglas Magde, James H. Brannon, Teresa L. Cremers, and John $01 \mathrm{mstead,} \mathrm{III,} \mathrm{"Absolute} \mathrm{Luminescence} \mathrm{Yield} \mathrm{of} \mathrm{Cresyl}$ Violet A Standard for the Red," Phys. Chem. 83 (6), 696-9 (22 March 1979).

190) E. K. Maldutis and S. V. Sakalauskas, "Contribution of thermoelastic stresses to the temperature coefficient of the refractive index $\mathrm{dn} / \mathrm{dT}$ of cubic crystals, "Sov. J. Quantum Electron 11 (9), 1255-7 (September 1981).

191) E. K. Maldutis and S. V. Sakalauskas, "Investigation of the kinetics of electrostrictive and thermal changes in the refractive index of glasses exposed to laser irradiation," Sov. J. Quantum Electron. 11 (9), 1244-6 (September 1981). 
192) A. Mandelis, "Absolute optical absorption coefficient measurements using transverse photothermal deflection spectroscopy," J. Appl. Phys. 54 (6), 3404-9 (June 1983).

193) A. F. Mastryukov and V. S. Synakh, "Thermal self-focusing of microwaves in a plasma," Fiz. Plazmy (USSR) \& (6), 1145-50 (Nov.-Dec. 1982) Trans. in Sov J. Plasma Phys.

194) A. F. Mastryukov and V. S. Synakh, "Thermal self-focusing of electromagnetic waves in a completely ionized plasma," $\mathrm{Zh}$. Prikl. Mekh. and Tekh Fiz. (USSR) 22 (5), 3-8 (Sept.-Oct. 1981) Trans. in J. Appl. Mech. and Tech. Phys. 22 (5), 596-601 (Sept.-Oct. 1981).

195) J. F. McClelland and R. N. Kniseley (Ames Lab., Ames, Iowa), "Photothermal conversion surface measurements using photothermal and photothermal spectroscopies," Proc. Semin. Test. Sol. Energy Mater. Syst., 245-52, Pub. Inst. Environ Sci., Mt. Prospect, IL.

196) F. A. McDonald, G. C. Wetsel, Jr., and S. A. Stotts, "Scanned photothermal imaging of subsurface structure, "Proc. of the 12th International Symposium on Acoustical Imaging held 19-22 July 1982 in London. Acoustical Imaging 12, 147-55 (Plenum Press, N.Y., 1982).

197) E. A. McLean, Louis Sica, and A. J. Glass, "Interferometric observation of absorption induced index change associated with thermal blooming," Appl. Phys. Lett. 13 (11), 369 (1 December 1968). 
198) Douglas McQueen, Per Helander and Ingemar Lundstrom (inventors), "Photothermal measurement cell for study of light absorption by a sample substance," European Pat. Appl. EP49918 Al, date: 820421 .

199) P. M. Mejias, "Light propagation through inhomogeneous media with radial refiactive index: Application to thermal blooming," Appl. Opt. 20 (24), 4287-95 (15 December 1981).

200) J. G. Mendoza-Alvarez, B. S. H. Royce, F. Sanchez-Sinencio, 0. Zelaya-Ange1, C. Menezes, and R. Triboulet, "Optical properties of cadmium telluride thin films studied by photothermal deflection spectroscopy," Thin Solid Films (Switzerland) 102, 259-63 (1983).

201) L. C. M. Miranda, "On the use of the thermal lens effect as a thermo-optical spectroscopy of solids," Appl. Phys. A 32 (Germany) (2), 87-93 (Oct. 1983).

202) Kazuhiko Miyaishi, Totaro Imasaka and Nobuhiko Ishihashi, "Thermal lens spectrometry based on image detection of a probe laser beam," Anal. Chem. 54 (12), 2039-44 (1982).

203) Kazuhiko Miyaishi, Totaro Imasaka and Nobuhiko Ishibashi, "Thermal lensing spectrophotometric analysis with ion-pair solvent extraction," Anal. Chim. Acta 124 (2), 381-9 (1981).

204) Yoshihiro Mizutani and Mikio Katayama, "Study of highly excited vibrational states of moiecules by thermal lens effects," Koen Yoshishu - Bunshi Kozo Sogo Toronkai 450-1 (1979): Chem. Soc. Japan (In Japanese). 
205) Kenji Mori, Totaro Imasaka and Nobuhiko Ishibashi, "Thermal lens spectroscopy based on pulsed laser excitation," Anal. Chem. 54 (12), 2034-8 (1982).

206) Kenji Mori, Totaro Imasaka and Nobuhiko Ishibashi, "Determination of nitrogen dioxide by pulsed thermal lens spectrophotometry," Anal. Chem. 55, 1075-9 (1983).

207) C. Morterra, M. J. D. Low and A. G. Severdia, "Some effects of specular and diffuse reflection on infrared photoacoustic spectra," Infrared Phys. (Great Britain) 22 (4), 221-7 (July 1982).

208) W. C. Mundy, J. E. L. Ermshar, P. D. Hanson, and R. S. Hughes, "Photothermal deflection microscopy of HR and AR coatings," Boulder Damage Symposium 1983.

209) W. C. Mundy, R. S. Hughes, and C. K. Carniglia, "Photothermal deflection microscopy of dielectric thin films, "Appl. Phys. Lett. 43 (11), 985-7 (1 Dec 1983).

210) Carl Glenn Murphy, "Thermal self-effects of laser beams in liquids," Dissertation U.N.M. 1970, Univ. Microfilms, Ann Arbor, MI, Order \#71-9317.

211) J. C. Murphy and L. C. Aamodt, "Photothermal spectroscopy using optical beam probing mirage effect," J. Appl. Phys. 51 (9), 4580-8 (1980).

212) J. C. Murphy and L. C. Aamodt, "Signal eniancement in photothermal imaging produced by three-dimensional heat flow," Appl. Phys. Lett. 39 (7), 519-21 (1981). 
213) G. Nolle, H. Ullrich, J. B. Muller, and J. Hesse, "A microprocessor controlled spectrometer for thermal scan Mossbauer spectroscopy," Nucl. Inst. and Meth. in Phys. Res. 207(3), 459 (1 Aprf1 1983).

214) Per Erik Nordal and Svein Otto Kanstad, "Phototherma 1 radiometry," Phys. Scr. 20 (5-6), 659-62 (1979).

215) Per Erik Nordal and Svein Otto Kanstad, "Visible-light speciroscopy by photochemical radiometry using an incoherent source," Appl. Phys. Lett. $\underline{38}$ (7), 486-8 (1981).

216) M. C. Oliveros, and D. R. Tilley, "Green functions and fluctuation spectra for coupled acoustic-thermal modes in semi-infinite isotropic media," J. Phys. Cl6(3), 473 (30 Jan. 1983).

217) M. A. Olmstead and N. M. Amer, "A new probe of the optical properties of surfaces," J. Vac. Sci. Technol. B 1, 751-3 (1983).

218) M. A. Olmstead and N. M. Amer, "Direct measurement of the polarization dependence of silicon (111) $2 \times 1$ surface-state absorption by use of photothermal displacement spectroscopy." Phys. Rev. Lett. $\underline{52}$ (13), 1148-51 (1984).

219) M. A. Olmstead and N. M. Amer, "Photothermal displacement spectroscopy: An optical probe for solids and surfaces," Appl. Phys. A 32, 141 (1983).

220) M. A. Olmstead, S. E. Kohn, and N. M. Amer, "Photothermal spectroscopy in vacuum," Bull. Am. Phys. Soc. 27, 227 (1982). 
221) J. Opsal, A. Rosencwaig, and D. L. Willenborg, "Thermal-wave detection and thin-film thickness measurements with laser beam deflection," Appl. Opt. 22 (20), 3169-76 (15 Oct 1983).

222) V. M. Orera and R. Alcala, "Photothermal bleaching of calcium colloids in additively colored calcium fluoride, "Solid State Commun. 27 (11), 1109-12 (1978).

223) Jean Oualid, Jacques Passavanti, Daniel Amingual, and Jacques Derrien, "Photothermal effect in the sinusoidal region," $C$. $R$. Acad. Sci., Paris Ser. A B 266B(14), 940-3 (1968) (In French).

224) J. H. Parks and D. A. Rockwell, "Surface studies with acoustic probe techniques," Laser Induced Damage in Optical Materials: 1975. NBS Special Publication 435, U. S. Dept. of Commerce, National Bureau of Standards, p. 157.

225) J. H. Parks and N. Alyassini, "Time resolved damage studies of thin films and substrate surfaces," Laser Induced Damage in Optical Materials: 1972 NBS Special Publication 372, U. S. Dept. of Commerce, National Bureaiu of Standards, p. 104.

226) J. K. Parks and Chen-Shui Tsai, "Thermal effects observed in ultrasonic diffraction experiments with retile crystals," Proc. IEEE 55(5), 701-2 (1967).

227) A. N. Pavlov, "Effect of an electrostatic field on the photothermal decolorization of $\mathrm{NaCl}$ crystals," Zh. Nauchn. Prikl. Fotogr. Kinematogr. 27 (1), 62-5 (1982) (In Russian).

228) Elzbieta Pawlik and Stanislaw Gebala, "Birefringence changes in barium-silicon glasses under the influence of photothermal processing," Appl. Opt. 2 (2), 115-9 (1979). 
229) C. R. Peatts and H. K. Wickramasinghe, "Phototherma 1 spectroscopy on a microscopic scale," Ultrason. Symp. Proc. , $_{\text {, }}$ 832-6 (1982).

230) R. F. Pierret and B. B. Roesner, "Photothermal probing of silicon-silicon oxide surface centers I and II," Solid State Electron. 19 (7), 577-91, 593-603 (1976).

231) K. V. Reddy, "Intracavity dye-laser photothermal deflection spectroscopy," Rev. Sci. Instrum. 54 (4), 422-4 (1983).

232) T. C. Rich and D. A. Pinnow, Appl. Phys. Lett. 20, 264 (1972).

233) Klaus E. Rieckhoff, "Self-induced divergence of $\mathrm{cw}$ laser beams in liquids -- A new nonlinear effect in the propagation of light," Appl. Phys. Lett. 9 (2), 87 (15 July 1966).

234) A. Rose, J. D. Pyrum, C. Muzny, G. J. Salamo and R. Gupta, "Application of the photothermal deflection technique to combustion diagnostics," Appl. Opt. 21 (15), 2663-5 (1 Aug. 1982).

235) C. B. Roxio, B. Abeles, C. R. Wronski, G. D. Cody, and T. Tiedje, "Comment on the optical absorption edge in hydrogenated amorphous silicon," Solid State Commun. 47 (12), 985-7 (1983).

236) B. S. H. Royce, F. Sanchez-Sinencio, R. Goldstein, R. Muratore, R. Williams and W. M. Yim, "Studies of photocorrosion at the zinc-selenide-electrolyte interface by photothermal deflection spectroscopy," J. Electrochem Soc. 129 (10), 2393-5 (1982). 
237) Shinsaku Sakamoto, Kiyokazu Tomi ie and Michinari Ito, "Two photon absorption spectroscopy aby using thermal lensing effects," Koen Yoshishu - Bunshi Kozo Sogo Toronkai, 222-3 (1979); Chem. Soc. Japan (In Japanese).

238) R. Santos and L. C. M. Miranda, "Theory of photothermal radiometry with solids," J. Appl. Phys. 52 (6), 4194-8 (1981).

239) Luise S. Schuetz, J. H. Cole, J. Jarzynski, N. Lagakos, and J. A. Bucaro, "Dynamic thermal response of single-mode optical fiber for interferometric sensors," Appl. Opt. 22(3), 478 (1983).

240) S. C. Seite1, J. O. Porteus, and W. N. Faith, "Measurement of optical coupling coefficients based on thermal expansion," Opt. Lett. 6 (12), 619-21 (December 1981).

241) D. Senderàkovà and P. Vojtek, "Thermal change of the refractive index of a linear absorber by an intensive light wave," Acta Physica Slovaca 33(2), 105 (1983).

242) S. J. Sheldon, L. V. Knight, and J. M. Thorne, "Laser-induced thermal lens effect: a new theoretical model," Appl. Opt. 21 (9), 1663-9 (1 May 1982).

243) Rongshen Sheng, Yanghuo Deng and Guanquan Chen, "Determination of heat by weak absorbing laser thermal lens," Wuhan Daxue Xuebao, Ziran Kexueban 3. 128 in Chinese (1981).

244) L. H. Skolnik, "A review of techniques for measuring small optical losses in infrared transmitting materials, "Optical Properties of Highly Transparent Solids, by Shashanka S. Mitra and Bernard Bendow, editors, 405 (Plenum Press, N.Y., 1975). 
245) L. H. Skolnik, B. Bendow, P. D. Gianino, A. Hordvik, and E. F. Cross, "Interference and lensing effects in the transmission of high power laser beams through infrared windows," Conf. on High Power Infrared Laser Window Materials, 30-31 October and 1 November 1972, AFCRL-TR-73-0372(I), Carl A. Pitha, editor, 15 (Air Force Cambridge Research Laboratories, L. G. Hanscom Field, Bedford, Massachusetts 01730, 19 June 1973).

246) A. Skumanich and N. M. Amer, "Optical properties and correlation energy of defects in hydrogenated amorphous germani um," J. Non-Cryst. Solids 59-60 (1), 249-52 (1983).

247) G. A. Smolenskii, A. K. Tagantsev, A. L. Kholkin, V. A. Trepakov, A. V. Davydov, "Study of the thermal polarization effect in ferroelectrics," Izvest. Akad. Nauk SSSR. Fiz. (Bul1. Acad. Sci. USSR, Phys.) 47(3), 598 (1983); Proc. 2nd All-union seminar on physics of ferroelastics-Voronezh, (1-3 April 1982).

248) Domenico Solimini, "Accuracy and sensitivity of the thermal lens method for measuring absorption," Appl. Opt. 5 (12), 1931 (December 1966).

249) Domenico Solimini, "Loss management of organic materials at 6328 \&," J. Appl. Phys. 37. 3314 (1966).

250) V. S. Soni and V. P. Nayyar, "Thermal focusing of a doughnut-shaped laser mode," J. Appl. Phys. 53 (2), 1212-4 (February 1982).

251) M. Sparks, "Optical distortion by heated windows in high-power laser systems," J. Appl. Phys. 42 (12), 5029 (November 1971). 
252) I. S. Spevak, "Thermal lens formation by radiation in a glass plate at softening temperature," Depov. Akad. Nauk Ukrsr. Ser. A (USSR) 8, 63-6 (1982) (In Ukranian).

253) I. S. Spevak, "Thermal lens in elastic and viscoelastic plates," Sov. J. Quantum Electiron. 11 (7), 863-8 (July 1981).

254) J. Stone, "Measurements of the absorption of light in low-loss liquids," J. Opt. Soc. America 62 (3), 327-33 (March 1972).

255) J. Stone, "Thermooptical technique for the measurement of absorption loss spectrum in liquids," Appl. Opt. $\underline{12}$ (8), 1828 (August 1973).

256) Robert L. Swofford, M. E. Long and A. C. Albrecht, "C-H vibrational states of benzene, naphthalene, and anthracene in the visible region by thermal lensing spectroscopy and the local mode model," J. Chem. Phys. 65 (1), 179 (1 July 1976).

257) Robert L. Swofford, M. E. Long, M. S. Burberry and A. C. Albrecht, "Free" $\mathrm{O}-\mathrm{H}$ overtone absorption of methanols in the visible region by thermal lens spectroscopy," J. Chem. Phys. 66 (2), 664 (15 January 1977).

258) Robert L. Swofford, Mitchell S. Burberry, James A. Morrell, and A. C. Albrecht, "C-H vibrational states of $\mathrm{C}_{6} \mathrm{D}_{5} \mathrm{H}$ and $\mathrm{C}_{6} \mathrm{~F}_{5}{ }^{\mathrm{H}}$ in the visible region by thermal lensing spectroscopy - A test of the local mode model," J. Chem. Phys. 66 (11), 5245 (1 June 1977). 
259) Robert L. Swofford and James A. Morre11, "Analysis of the repetitively pulsed dual-beam thermo-optical absorption spectrometer," J. Appl. Phys. 49 (7), 3667-73 (July 1978).

260) A. C. Tam and B. Sullivan, "Remote sensing applications of pulsed photothermal radiometry," Appl. Phys. Lett. 43 (4), 333-5 (15 Aug 1983).

261) Keiji Tanaka, Ryoh Satoh, and Akira Odajima, "Photothermal spectrosocpy of thin solid films," Japan J. Appl. Phys., Part $2 \underline{22}$ (9), 592-4 (Sept. 1983) (In English).

262) K. Tenna 1, A. Rose, J. Pyrum, C. Muzny, R. Gupta, and G. Salamo, "Minority species concentration measurements in flames using the photoacoustics and photothermal deflection techniques," Proc. Int. Conf. Lasers (1981), 1012-19 (1982).

263) Gareth Thomas and B. L. Sopori, "Thermal lens effect in cadmi um sulfide," J. Appl. Phys. 4l(2), 603-8 (1970).

264) Roderick D. Tom, Ellen P. O'Hara and David Benin, "A generalized model of phototherna! radiometry," J. Appl. Phys. 53 (8), 5392-400 (1 Aug. 1982).

265) Roderick Tom, T. A. Moore, S. H. Lin and D. Benin, "A thermal diffusion model of photothermal spectroscopy," Chem. Phys. Lett. 66 (2), 390-4 (1979).

266) R. M. Toussaint and V. Jaccarino, "Photothermal detection of magnetic polaritons in ferrous fluoride," J. Appl. Phys. $\underline{55}$ (6), Part 2B, 2458-60 (1984). 
267) Y. F. Tsay, H. G. Lipsor and P. A. Ligor, "The temperature variation of the thermo-optic coefficient of calcium fluoride," J. Appl. Phys. 48 (5), 1593-5 (1977).

268) Allen John Twarowski, "Two-photon thermal blooming spectroscopy of benzene and 1,3,5-hexatriene and excited state spectroscopy of $\alpha, w$-diphenylpolyenes," PhD Dissertation, U.C. Santa Cruz (1976), Univ. Microfilms Int. Order No. 77-16, 883.

269) A. J. Twarowski and D. S. Kliger, "Multiphoton absorption spectra using thermal blooming I. Theory," Chem. Phys. 20 (2), 253-8 (1 March 1977).

270) A. J. Twarowski and D. S. Kliger, "Multiphoton absorption using thermal blooming II. Two-photon spectrum of benzene," Chem. Phys. 20 (2), 259-64 (1 March 1977).

271) A. J. Tyarowski and D. S. Kliger, "A search for a low-lying excited A state in 1, 3, 5 hexatriene," Chem. Phys. Lett. 50 (1), 36-40 (15 August 1977).

272) Veronicé Vaida, John L. Casey, and Steven D. Colson, "Multiphoton transitions in trans-butadiene observed by multiphotor ionization and thermal lens spectroscopy." Chem. Phys. Lett. $\underline{54}$ (1), 25 (15 February 1978).

273) V. Vaida, M. B. Robin and N. A. Kuebler, "A note on the elusive ${ }^{E_{2 g}}$ state in the two-photon spectrum of benzene," Chem. Phys. Lett. 58 (4), 557 (15 October 1978). 
274) A. A. Vigasin, Yu A. Gorokhov, A. P. Sukhorukov and A. M. Khachatryan, "Nonlinear spherical aberrations and thermo-elastic stresses during thermal self-focusing," Zh. Tekh. Fiz. 44 (5), 1070-5 in Russian (1974).

275) V. Volterra and Weiner Avnear, "Continuous wave thermal lens effect in thin layer of nematic liquid crystal," Opt. Commun. 12 (2), 194-7 (1974).

276) V. V. Vorob'yev, "Evaluations of the possibilities of compensating the thermal defocusing of laser beams by means of phase conjugate adaptive systems," Radiotekh and Electron (USSR) 26 (11), 2334-41 (Nov. 1981), Trans. in: Radio Eng. and Electron. Phys. 26 (11), 63-9 (Nov. 1981).

277) Grover C. Wefsel, Jr. and Alan F. McDonald, "Subsurfacestructure determination using photothermal laser-beam deflection," Appl. Phys. Lett. 41 (10), 926-8 (15 Nov. 1982).

278). John R. Whinnery, "Laser measurement of optical absorption in liquids," Accts. Chem. Research I (7), 225-31 (July 1974).

279) J. R. Whinnery, D. T. Miller, and F. Dabby, "Thermal convection and spherical aberration distortion of laser beams in low-loss liquids," IEEE J. Quantum Electron $\underline{3}$ (9), 382-3 (September 1967).

280) R. Wilbrandt and $H$. Weber, "Effect of thermal blooming in a liquid filter on the emission of neodymium glass laser," Opt. Commun. 9 (3), 231-3 (1972). 
281) E. Wild and Max Majer, "Thermal blooming in liquid nitrogen during high repetition rate stimulated Raman scattering." J. Appl. Phys. 51 (6), 3078-80 (1980).

282) K. E. Wilson and L. G. deShazer, "Dispersion of thermo-optic coefficients of neodymium laser materials,". NBS Spec. Publ. (U.S.) 574, 221-2 (1980).

283) J. S. Wong, "FTIR photothermal deflection spectroscopy," IBM Tech. Disclosure Bu11. 26 (1), 345-6 (June 1983).

284) Ma Xing-Xiao and Xu Zhu-De, "Studies of $V-T / R$ relaxation in polyatomic molecules with a time-resolved thermal lens," Chem. Phys. Lett. (Netherlands) 98 (6), 563-5 (15 July 1983).

285) Zhude $X U$ and Xingxiao Ma (Dep. Chem. Chinese U. Sci. Technol, Hefei) "Measurements of vibrational relaxation rate for polyatomic molecules with time-resolved thermal lens," Fenzi Kexue Xuebao 1 (1), 121-2 (1981) (In Chinese).

286) Takeshi Yamada and Tokashi Kurokawa, "Light beam deflection in polymer films by dielectric loss heating," Japan J. Appl. Phys. Part $1 \underline{21}$ (12), 1746 (Dec. 1982) (In English).

287) Qi Yao, "Photothermal deflection spectroscopy," Wuli $\underline{12}$ (8), 462-5 (1983) (in Chinese).

288) Zafer A. Yasa, Warren B. Jackson and Nabil M. Amer, "Photothermal spectroscopy of scattering media," App1. Opt. 21 (1), 21-31 (1982). 
289) William M. Yen, "Photoacoustic and photothermal spectroscopy of ions in solids," Report ARO-13462.4-P (1978) Order \#AD-A066063, NTIS.

290) L. D. Ziegler and B. S. Hudson, "The thermal biooming spectrum of liquid benzene in the near ultraviolet," Chem. Phys. Lett. 기 (1), 113-16 (1980).

291) G. M. Zverev, E. A. Levchuk, E. K. Maldutis, and V. A. Pashkov, "Thermal self-focusing of laser radiation in substances with negative $\mathrm{dn} / \mathrm{dT}, " \mathrm{Pis}$ 'ma Zh. Eksp. Teor. Fiz. 11(3), 177-81 (1970 (In Russian).

292) G. M. Zverev, E. A. Levchuk, E. Maldutis, and V. A. Pashkov, "Thermal self-focusing of radiation from a free-running laser in KDP and ADP." Zh. Eksp. Teor. Fiz. 58(5), 1487-90 (1970) (In Russian). 
Figure 4-5. Comparison of Present and Previous Theories . . . . . . 126

Figure 4-6. Theoretical Values and Measured Thermal Lensing Signal . . . . 127

Figure 5-1. Photothermal Lensing Experiment . . . . . . . . . 135

Figure 5-2. Temporal Pulse Shape of the Probe Beam at $532 \mathrm{~nm} \ldots \ldots \ldots 137$

Figure 5-3. Distribution of Fluence in the Pump Beam $\ldots \ldots \ldots \ldots \ldots 139$

Figure 5-4. Spatial Profile of the HeNe Probe Beam . . . . . . . . . 141

Figure 5-5. Encircled Power in the HeNe Probe Beam . . . . . . . . 143

Figure 5-6. Optical Beam Analysis System . . . . . . . . . . . 144

Figure 5-7. Thermal Lensing Signal in $\mathrm{ZnS}$ at $580 \mathrm{~nm} \ldots \ldots \ldots \ldots \ldots 152$

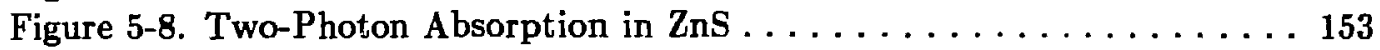

Figure 5-9. Two-Photon Absorption in $\mathrm{TiO}_{2}$ (Rutile) . . . . . . . . 159

Figure 5-10. Linear Optical Absorption Near the Band Edge in

Several Glasses . . . . . . . . . . . . . . . . 163

Figure 5-11. Thermal Lensing Signal in Several Silicate Glasses . . . . . 166

Figure 5-12. Thermal Lensing Signal in Several Borosilicate

Glasses and One Phosphate . . . . . . . . . . . . . . 167

Figure 5-13. Two-Photon Absorption Coefficient in BK7 at Several

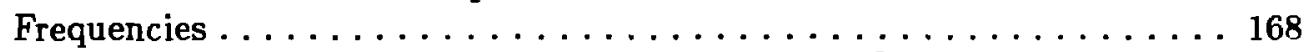

Figure 5-14. Typical Linear Absorption spectrum of $\mathrm{Nd}^{3+} \ldots \ldots \ldots \ldots 173$

Figure 5-15. Thermal Relaxation of $\mathrm{Nd}^{3+} \ldots \ldots \ldots \ldots \ldots \ldots \ldots \ldots$

Figure B-1. Face-Centered Cubic Lattice . . . . . . . . . . . . 194

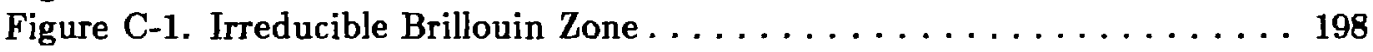

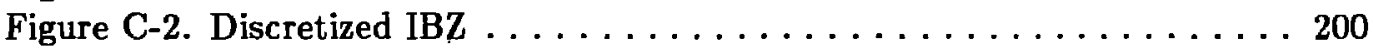

List of Tables

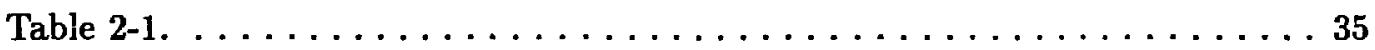

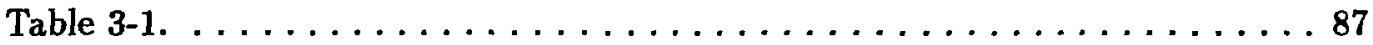

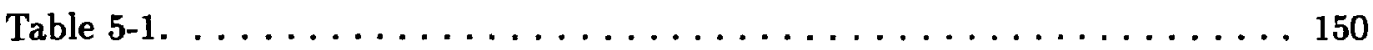

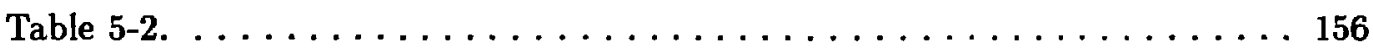

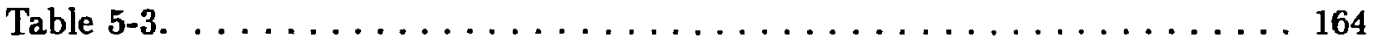

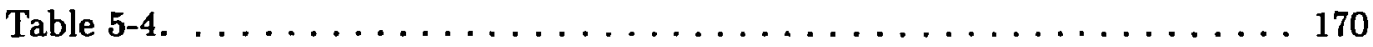

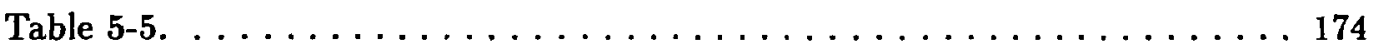

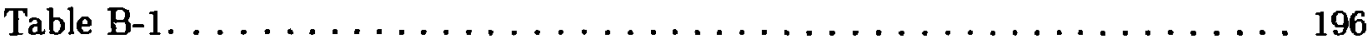

